\title{
Neural Machine Translation
}
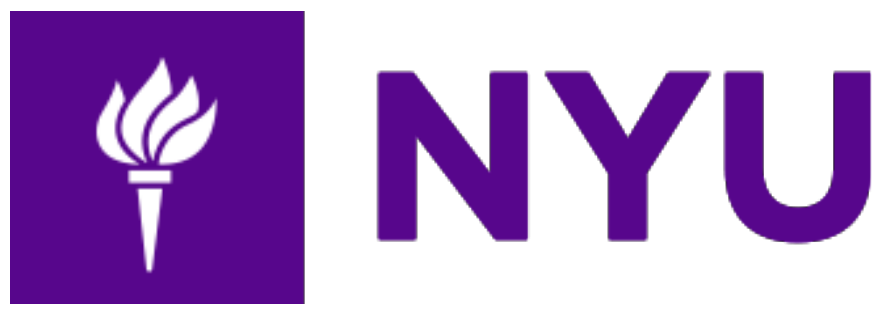

\section{Stanford}

Thang Luong

Kyunghyun Cho

Christopher Manning

@lmthang·@kchonyc·@chrmanning

ACL 2016 tutorial · https://sites.google.com/site/acl16nmt/ 
1a. Intro to (Neural) Machine Translation Ideas connecting Phrase-Based Statistical MT and NMT Neural Language Models 


\section{Machine Translation}

The classic test of language understanding!

Both language analysis \& generation

Big MT needs ... for humanity ... and commerce

Translation is a US\$40 billion a year industry

Huge in Europe, growing in Asia

Large social/government/military

as well as commercial needs






\section{The need for machine translation}

Huge commercial use

Google translates over 100 billion words a day

Facebook has just rolled out new homegrown MT

"When we turned [MT] off for some people, they went nuts!"

eBay uses MT to enable cross-border trade 


\section{Scenarios for machine translation}

1. The dream of fully automatic high-quality MT (FAHQMT)

This still seems a distant goal

2. User- or platform-initiated low quality translation

The current mainstay of MT

Google Translate

Bing Translator 


\section{Scenarios for machine translation}

3. Author-initiated high quality translation

MT with human post-editing or MT as a translation aid is clearly growing ... but remains painful

Great opportunities for a much brighter future where MT assists humans: e.g., MateCat or LiLT

https://lilt.com/
Talk in Sess $1 C$ !
Durante nuestro período de pruebas, Lilt es completamente gratuito y no tiene límites de uso.

During our trial period, Lilt is completely free and has no| 
Progress in MT

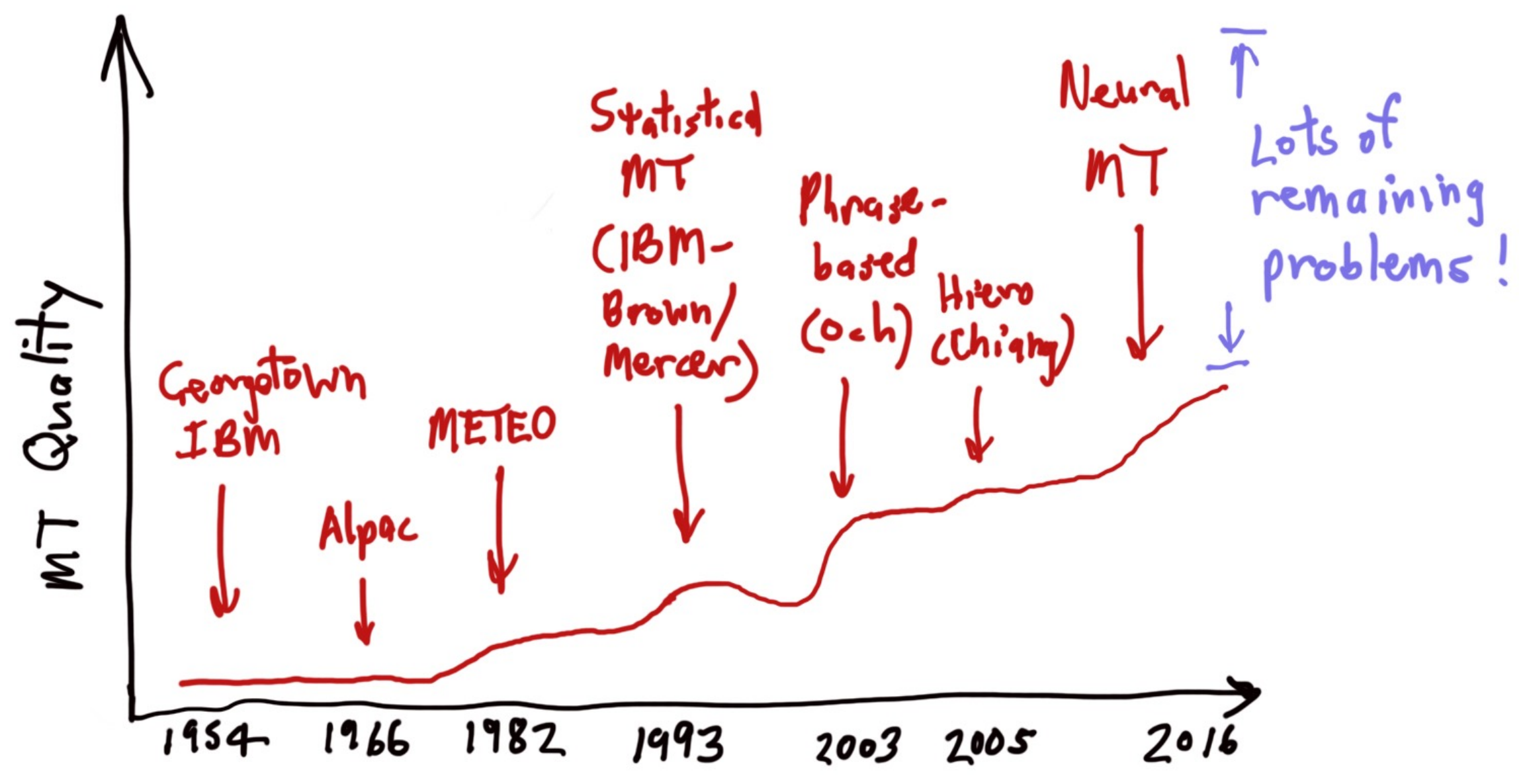

7 


\section{Graham Neubig}

Wow, @stanfordnlp 's neural MT system for the IWSLT en-de task outperforms 2 nd place by a massive 4.7 BLEU points: workshop2015.iwslt.org/downloads/IWSL... 12/3/15, 7:18 PM

12 RETWEETS 21 LIKES 


\section{IWSLT 2015, TED talk MT, English-German}

\section{BLEU (CASED)}

35

30

30

25

20

15

10

5

0 30.85

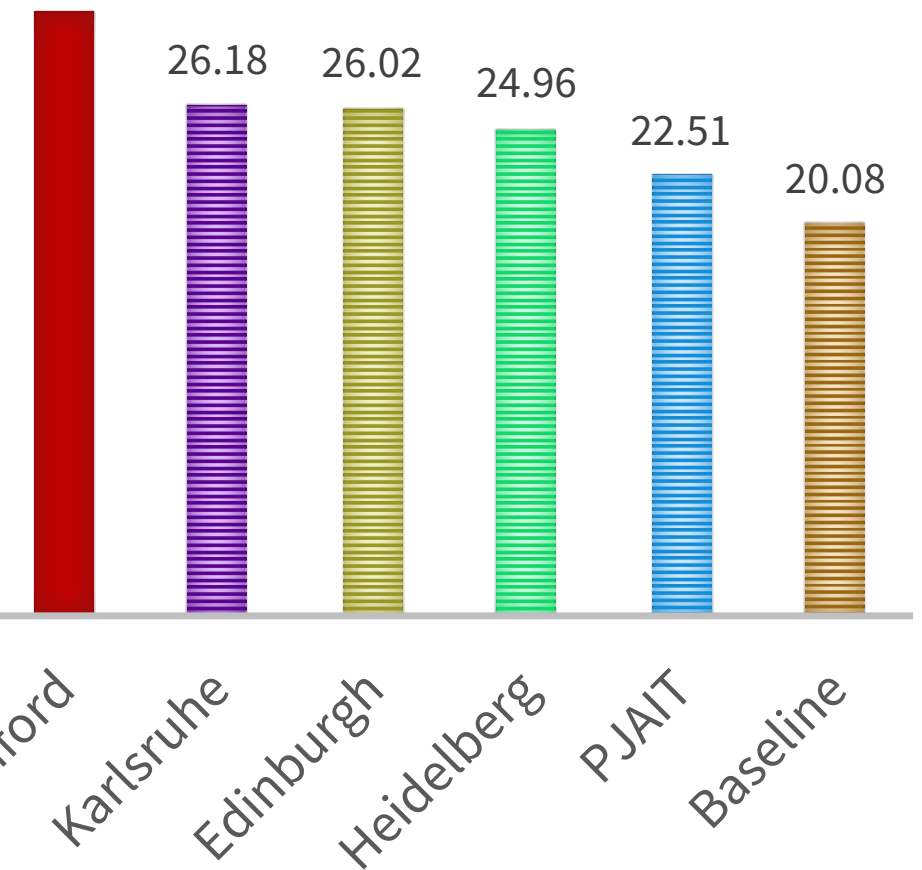

HUMAN EVALUATION

(HTER)

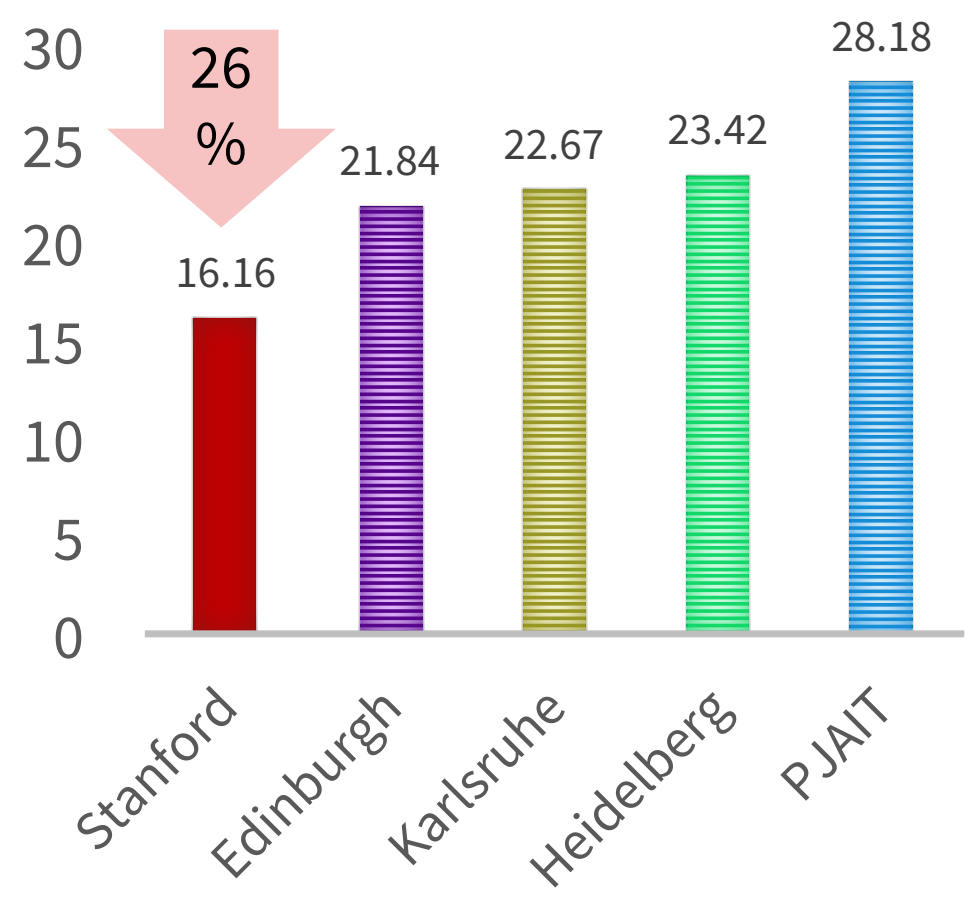




\section{Progress in Machine Translation}

[Edinburgh En-De WMT newstest2013 Cased BLEU; NMT 2015 from U. Montréal]

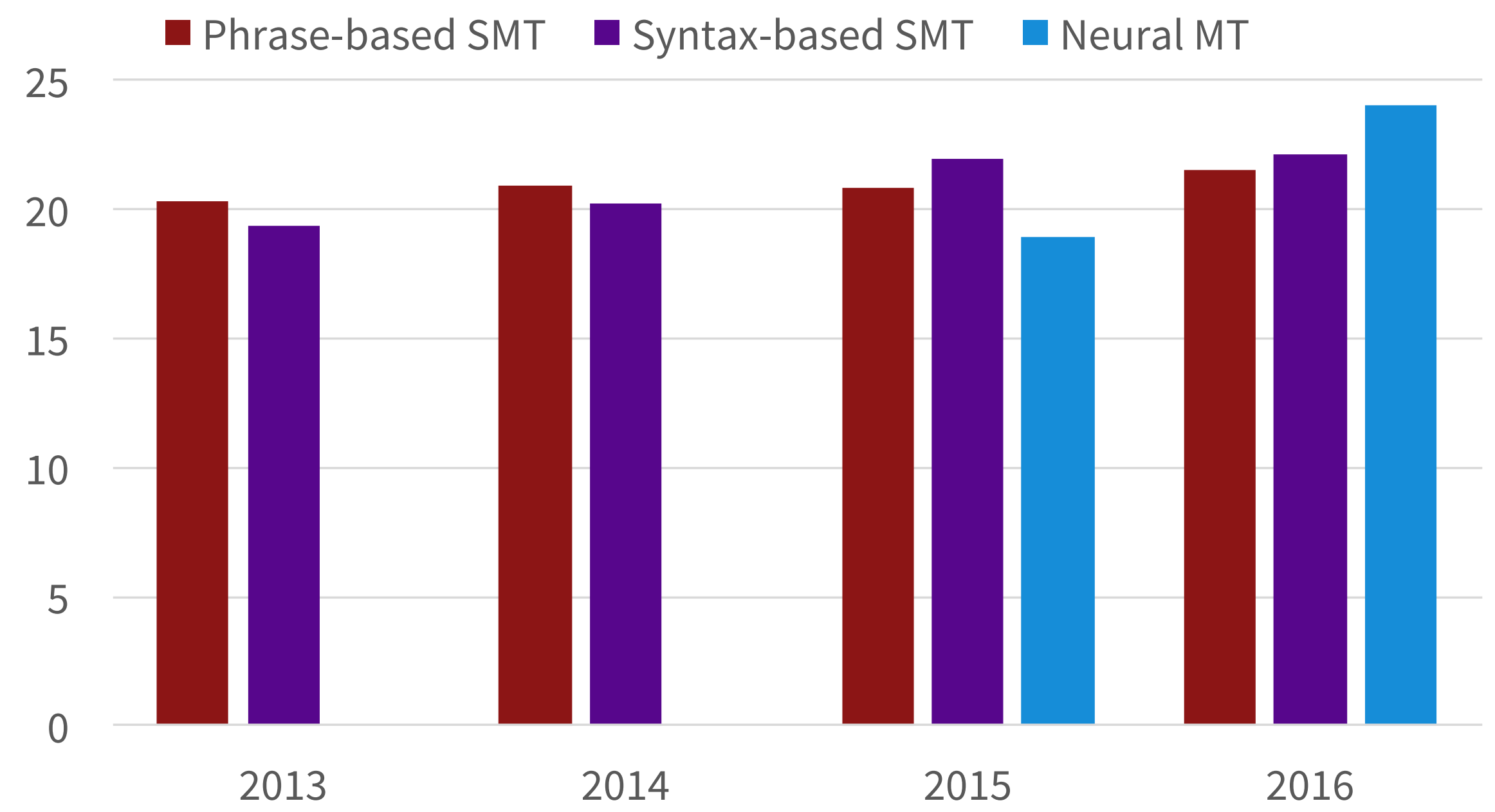

From [Sennrich 2016, http://www.meta-net.eu/events/meta-forum-2016/slides/09_sennrich.pdf] 


\section{Phrase-based Statistical Machine}

Translation

\section{A marvelous use of big data but ... it's mined out?!?}

\section{9年600名西班牙人在墨西哥登陆，去征服几百万 \\ 人口的阿兹特克帝国，

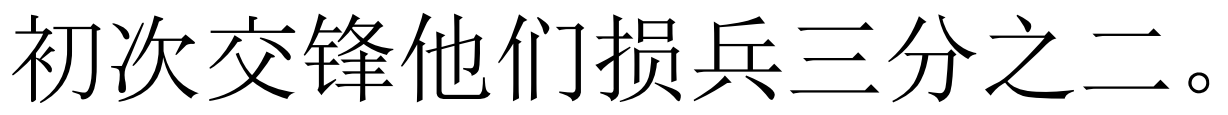

In 1519, six hundred Spaniards landed in Mexico to conquer the Aztec Empire with a population of a few million. They lost two thirds of their soldiers in the first clash.

translate.google.com (2009): 1519600 Spaniards landed in Mexico, millions of people to conquer the Aztec empire, the first two-thirds of soldiers against their loss.

translate.google.com (2013): 1519600 Spaniards landed in Mexico to conquer the Aztec empire, hundreds of millions of people, the initial confrontation loss of soldiers two-thirds.

translate.google.com (2014): 1519600 Spaniards landed in Mexico, millions of people to conquer the Aztec empire, the first two-thirds of the loss of soldiers they clash.

translate.google.com (2015): 1519600 Spaniards landed in Mexico, millions of people to conquer the Aztec empire, the first two-thirds of the loss of soldiers they clash.

translate.google.com (2016): 1519600 Spaniards landed in Mexico, millions of people to conquer the Aztec empire, the first two-thirds of the loss of soldiers they clash. 
Neural MT is good! 
Neural MT went from a fringe research activity in 2014 to the widely-adopted leading way to do MT in 2016 .

Amazing! 
What is Neural MT (NMT)?

Neural Machine Translation is the approach of modeling the entire MT process via one big artificial neural network*

*But sometimes we compromise this goal a little 


\section{Neural encoder-decoder architectures}

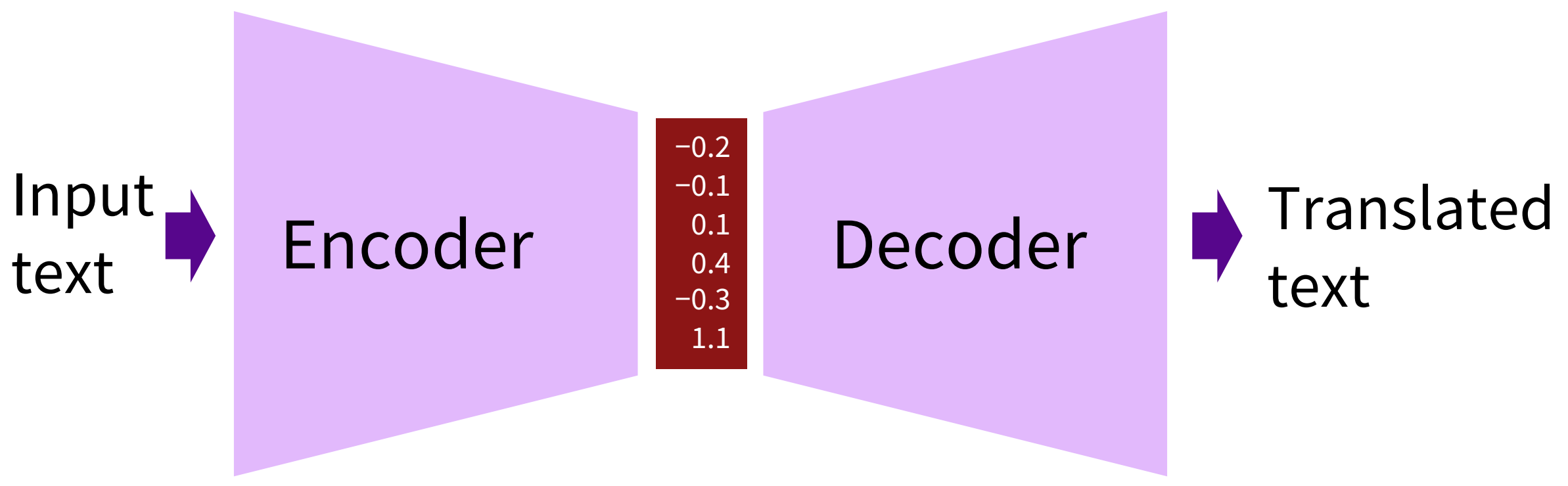




\section{NMT system for translating a single word}

$\begin{array}{ccc}V_{s} \times 1 & d \times V_{s} & d \times 1 \\ w & h & x=h w\end{array}$

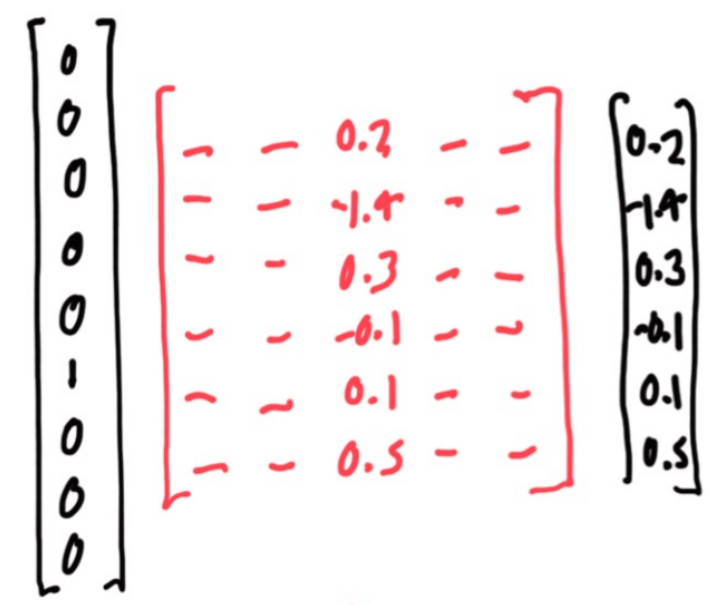

$\uparrow$
ane hot Looks up
word column of
symbol word embedding
matrix

word 
NMT system for translating a single word

Nowolineanity $f=-1$

$$
\begin{aligned}
& \begin{array}{ccccc}
V_{s} \times 1 & d x V_{s} & d x 1 & d x d & d x 1 \quad a=f(z) \\
w & h & x=h w & A & z=A x+b d x 1
\end{array}
\end{aligned}
$$

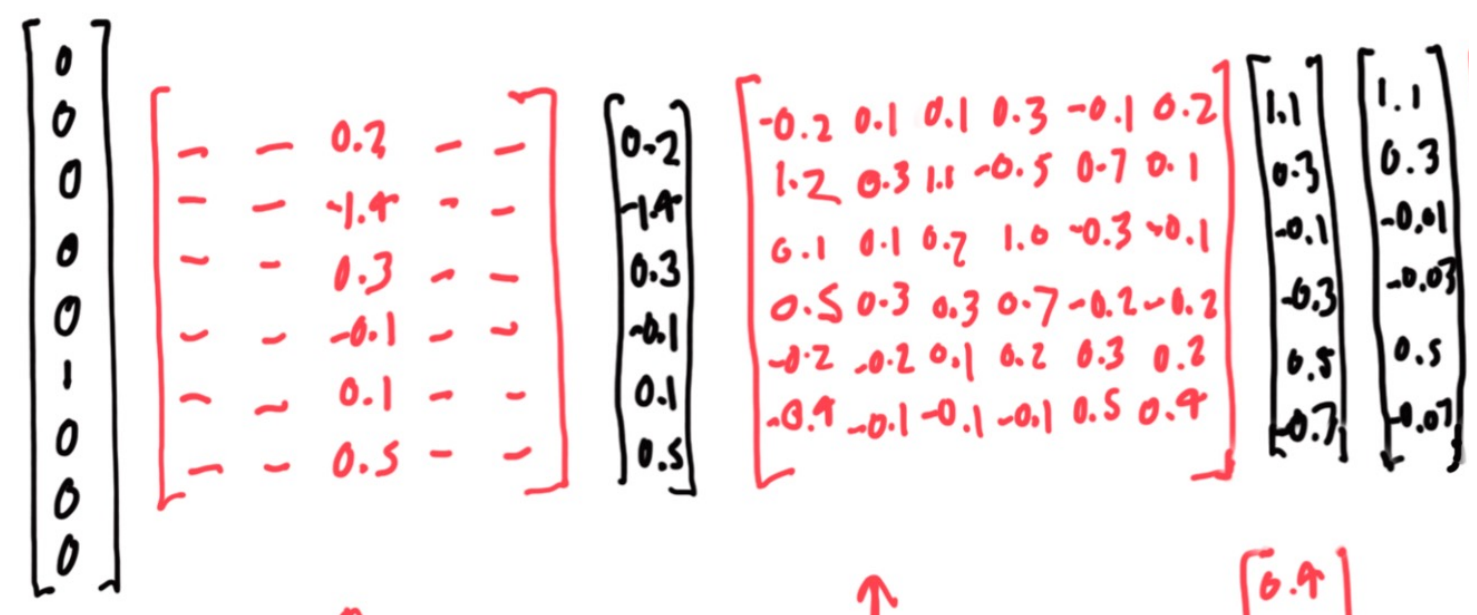

$$
\begin{aligned}
& \left.\begin{array}{ccc}
1 & \uparrow & \uparrow \\
\text { one hot Looks up } & \text { Transformation } \\
\text { word column of } & \text { Matrix maps } \\
\text { symbol word embedding } & \begin{array}{l}
0.9 \\
0.1 \\
-0.3 \\
\text { matrix }
\end{array} \\
& \text { vector space } & -0.2 \\
0.1 \\
0.2
\end{array}\right] \\
& \left.\begin{array}{ccc}
1 & \uparrow & \uparrow \\
\text { one hot Looks up } & \text { Transformation } \\
\text { word column of } & \text { Matrix maps } \\
\text { symbol word embedding } & \begin{array}{l}
0.9 \\
0.1 \\
-0.3 \\
\text { matrix }
\end{array} \\
& \text { vector space } & -0.2 \\
0.1 \\
0.2
\end{array}\right] \\
& \uparrow
\end{aligned}
$$

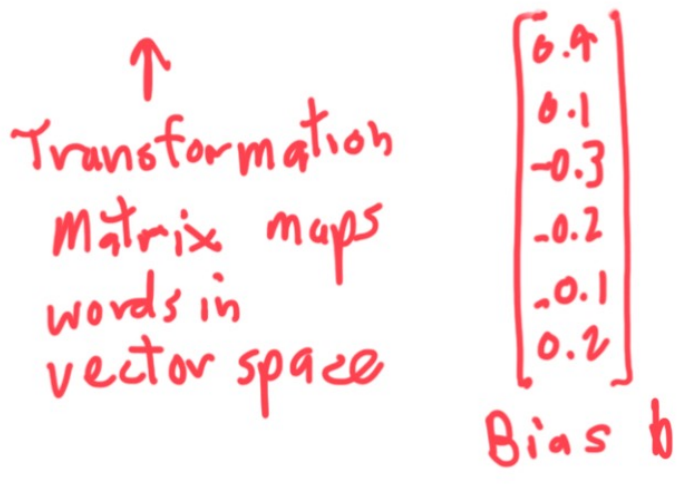


NMT system for translating a single word

$$
\begin{aligned}
& \text { Nownlineanity } f=\ldots \\
& \text { Softmax } \\
& p_{i}=\frac{e^{u_{i}}}{\sum_{j} e^{u_{j}}}
\end{aligned}
$$

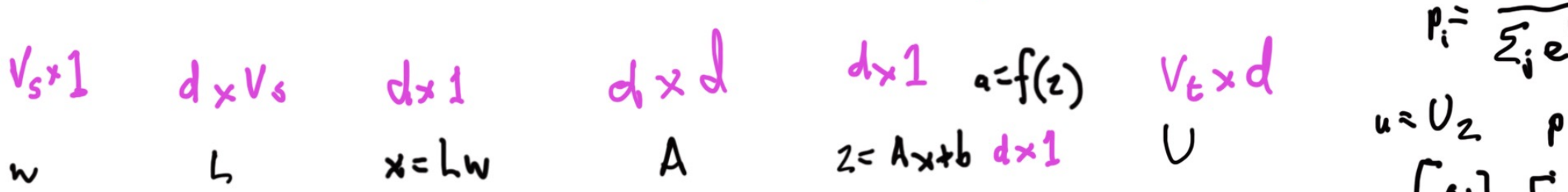

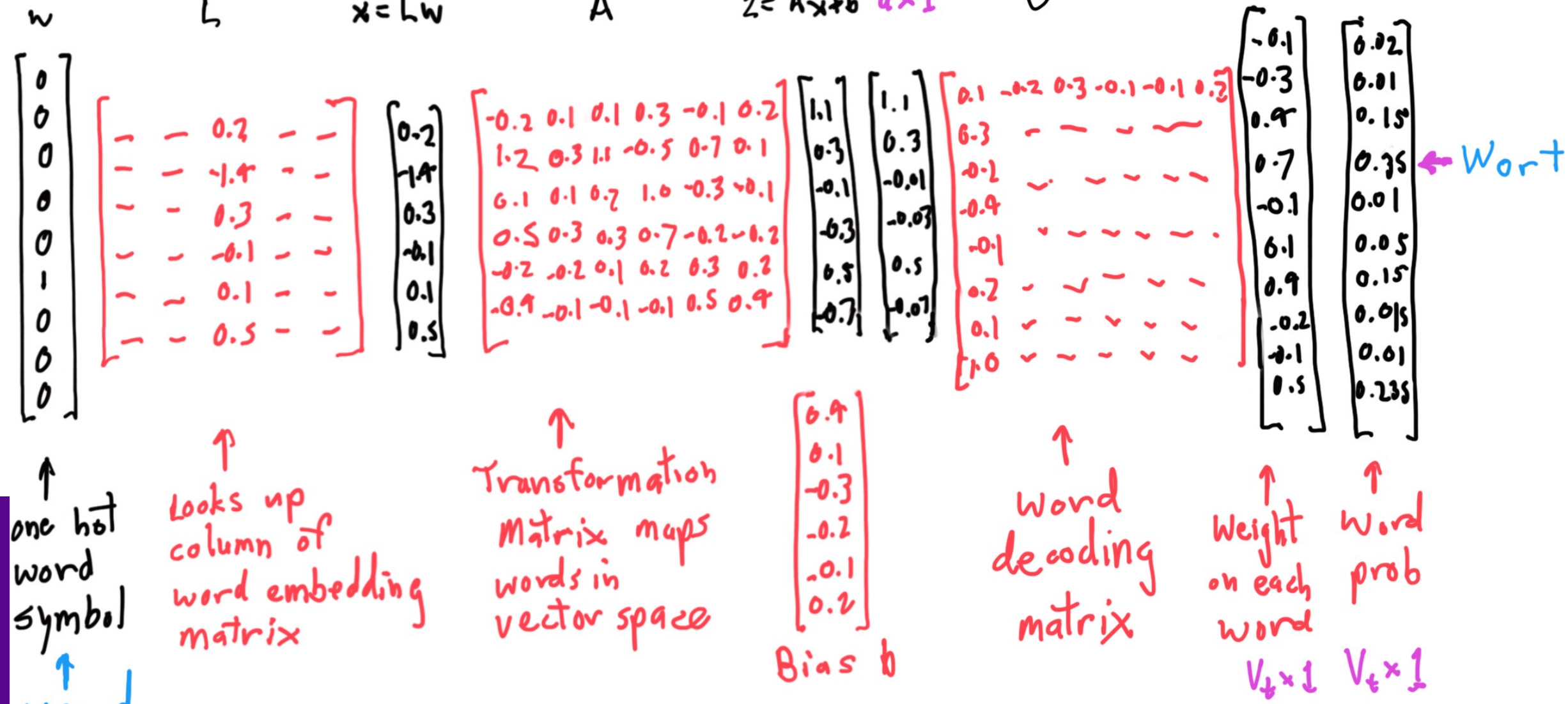


Softmax function: Standard map from $\mathbb{R}^{V}$ to a probability distribution

Exponentiate to

Soft max make positive

Normalize to give probability

$$
p_{i}=\frac{e^{u_{i}}}{\sum_{j} e^{u_{j}}}
$$




\section{Neural MT: The Bronze Age}

[Allen 1987 IEEE $1^{\text {st }}$ ICNN]

3310 En-Es pairs constructed on 31 En, 40 Es words, max $10 / 11$ word sentence; 33 used as test set

The grandfather offered the little girl a book $\rightarrow$ El abuelo le ofrecio un libro a la nina pequena Binary encoding of words - 50 inputs, 66 outputs; 1 or 3 hidden 150-unit layers. Ave WER: 1.3 words

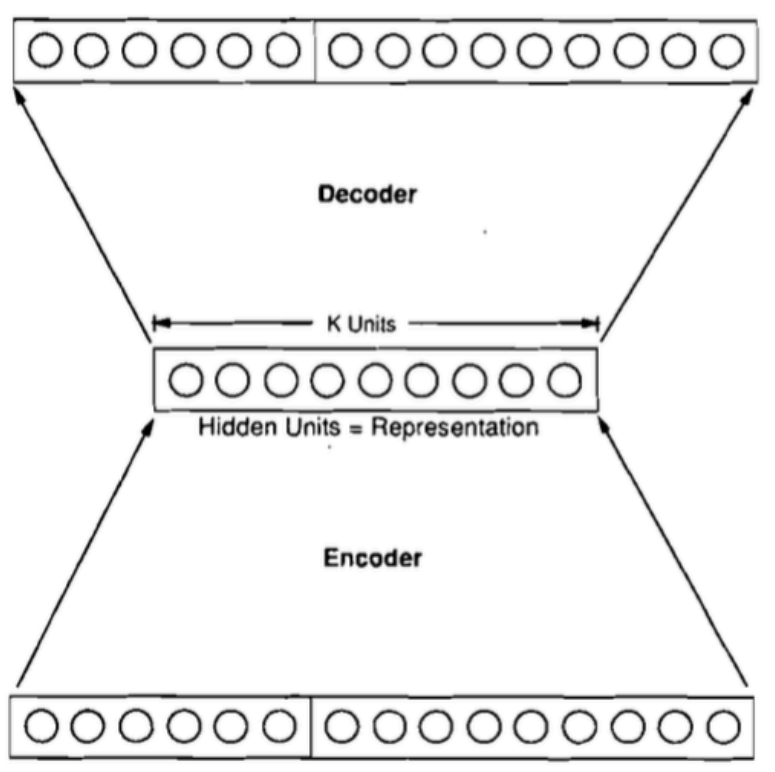




\section{Neural MT: The Bronze Age}

[Chrisman 1992 Connection Science]

Dual-ported RAAM architecture [Pollack 1990 Artificial Intelligence] applied to corpus of 216 parallel pairs of simple En-Es sentences:

You are not angry $\leftrightarrow$ Usted no esta furioso

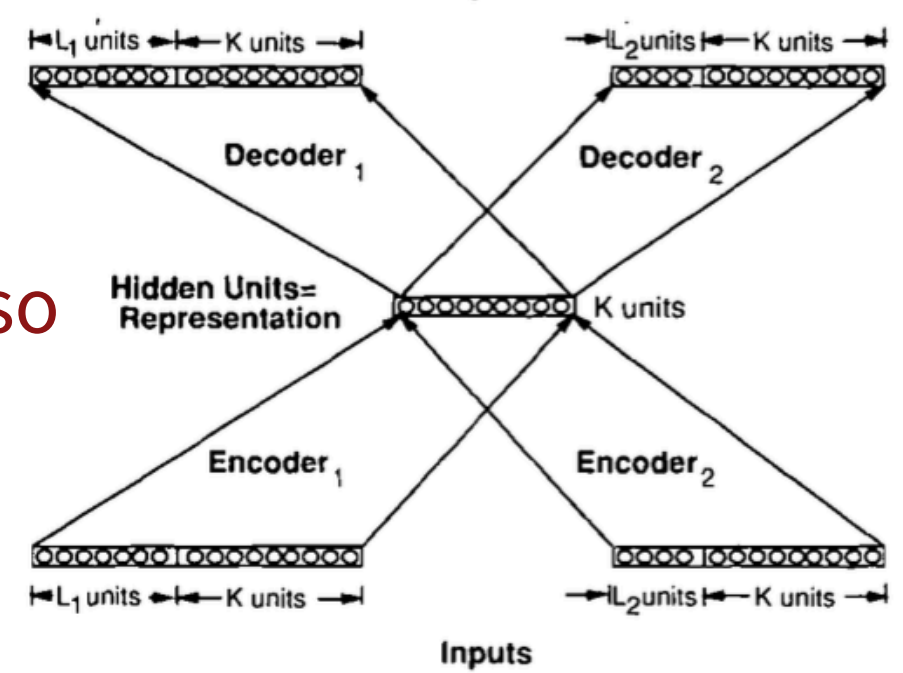




\section{Coincidence?}

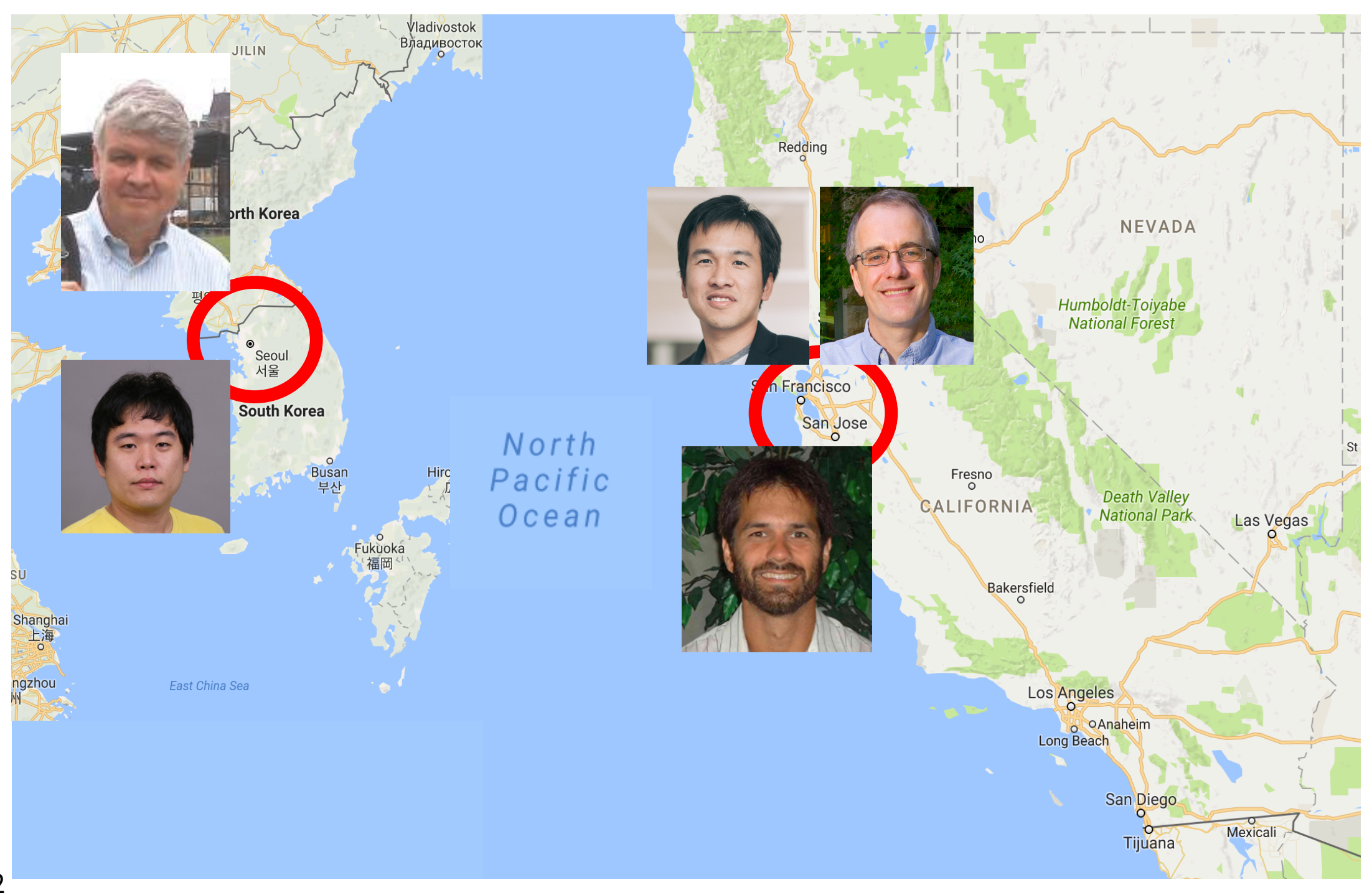




\section{Modern Sequence Models for NMT}

[Sutskever et al. 2014, Bahdanau et al. 2014, et seq.] following [Jordan 1986] and more closely [Elman 1990]

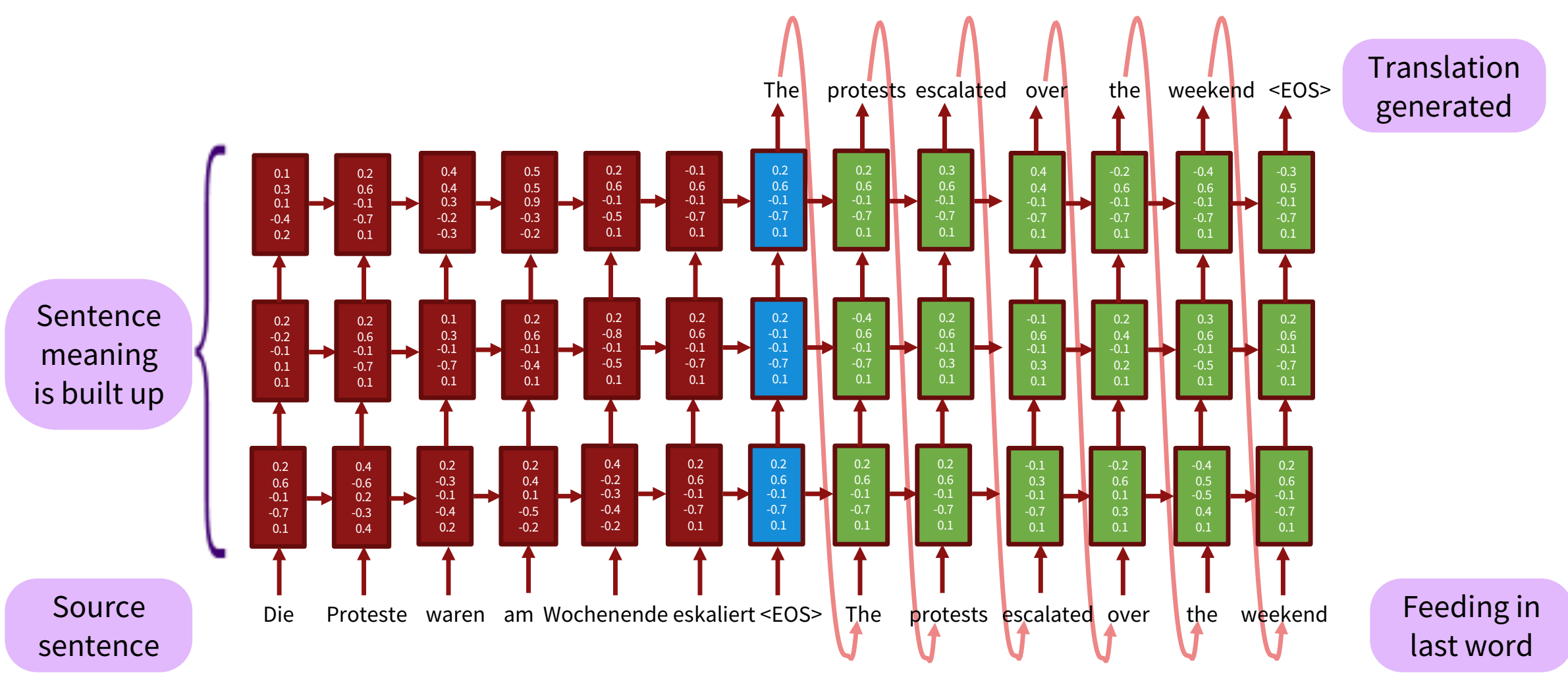

A deep recurrent neural network 


\section{The three big wins of Neural MT}

1. End-to-end training

All parameters are simultaneously optimized to minimize a loss function on the network's output

2. Distributed representations share strength Better exploitation of word and phrase similarities

3. Better exploitation of context

NMT can use a much bigger context - both source and partial target text - to translate more accurately 
What wasn't on that list?

1. Explicit use of syntactic or semantic structures

2. Explicit use of discourse structure, anaphora, etc.

3. Black box component models for reordering, transliteration, etc. 
The current baseline and its enduring ideas

1b. Ideas connecting Phrase-Based Statistical MT and NMT 


\section{Word alignments}

Phrase-based SMT aligned words in a preprocessing-step, usually using EM

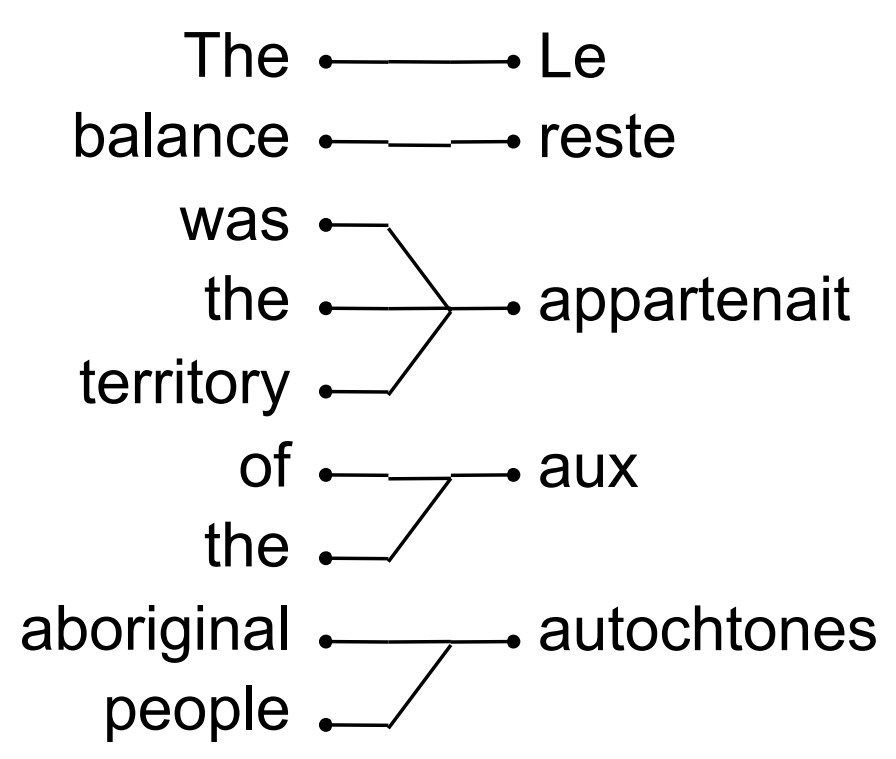

$\rightarrow$ Models of attention

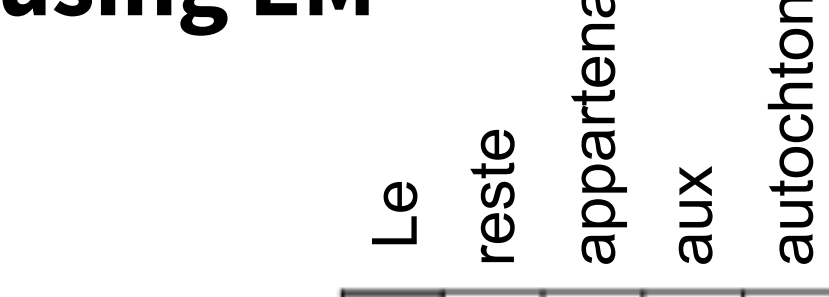

[Bahdanau et al. 2014; ICLR 2015]

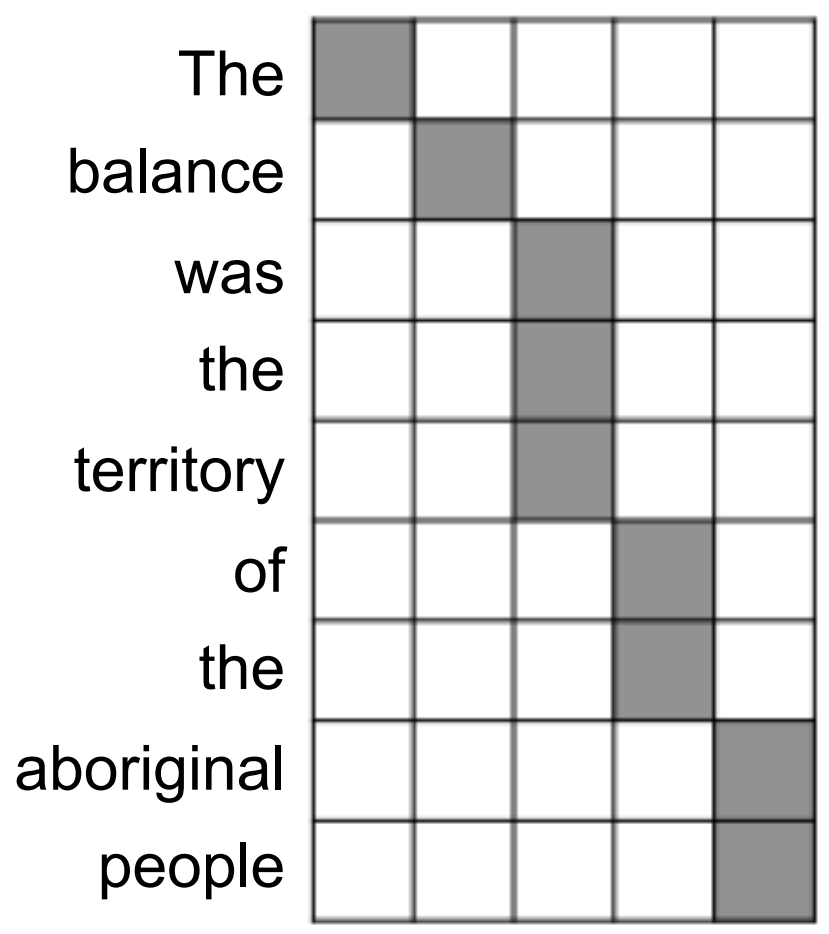

Part 3b later 


\section{Constraints on "distortion" \\ (displacement) and fertility}

SMT: Alignment probability depends on positions of the words, and position relative to neighbors

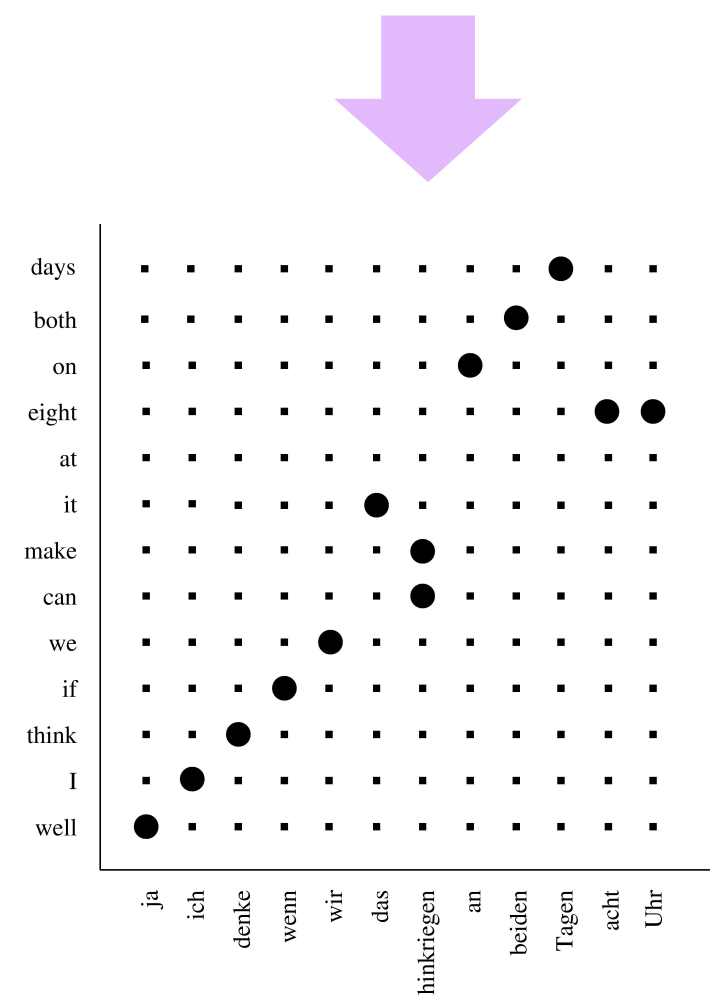

\section{The likelihood of an alignment depends on how many words align to a certain position}

farmers

\begin{tabular}{|cr|rr|}
\hline $\mathrm{f}$ & $t(f \mid e)$ & $\phi$ & $n(\phi \mid e)$ \\
\hline agriculteurs & 0.442 & 2 & 0.731 \\
les & 0.418 & 1 & 0.228 \\
cultivateurs & 0.046 & 0 & 0.039 \\
producteurs & 0.021 & & \\
\hline
\end{tabular}

the

\begin{tabular}{|rr|}
\hline$\phi$ & $n(\phi \mid e)$ \\
\hline 1 & 0.746 \\
0 & 0.254 \\
\hline
\end{tabular}




\section{Constraints on "distortion" (displacement) and fertility}

$\rightarrow$ Constraints on attention [Cohn, Hoang, Vymolova, Yao, Dyer \& Haffari NAACL 2016; Feng, Liu, Li, Zhou 2016 arXiv; Yang, Hu, Deng, Dyer, Smola 2016 arXiv].

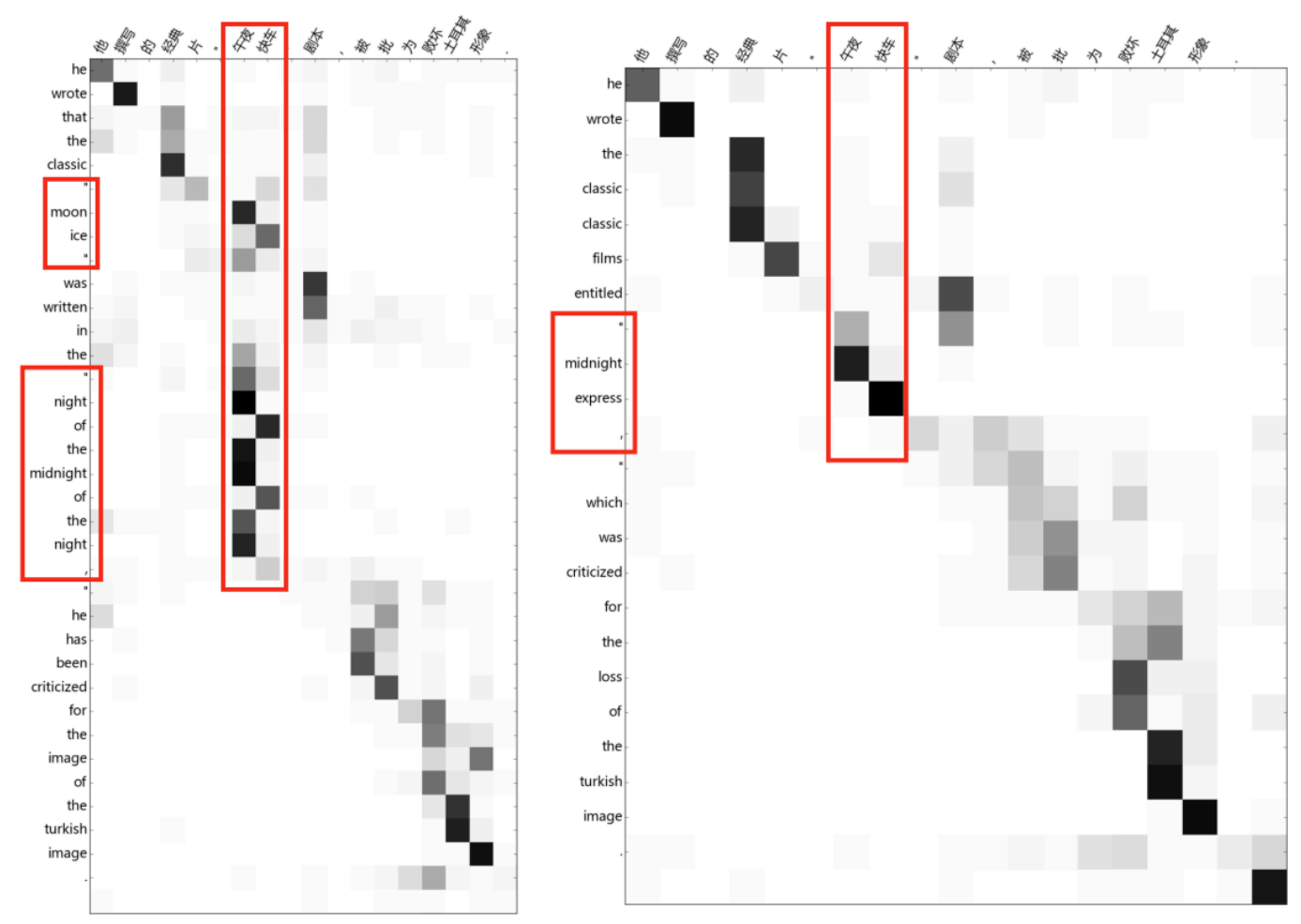




\section{Automatic evaluation method for learning Before usually BLEU; NMT $\rightarrow$ usually differentiable LM score, i.e., predict each word}

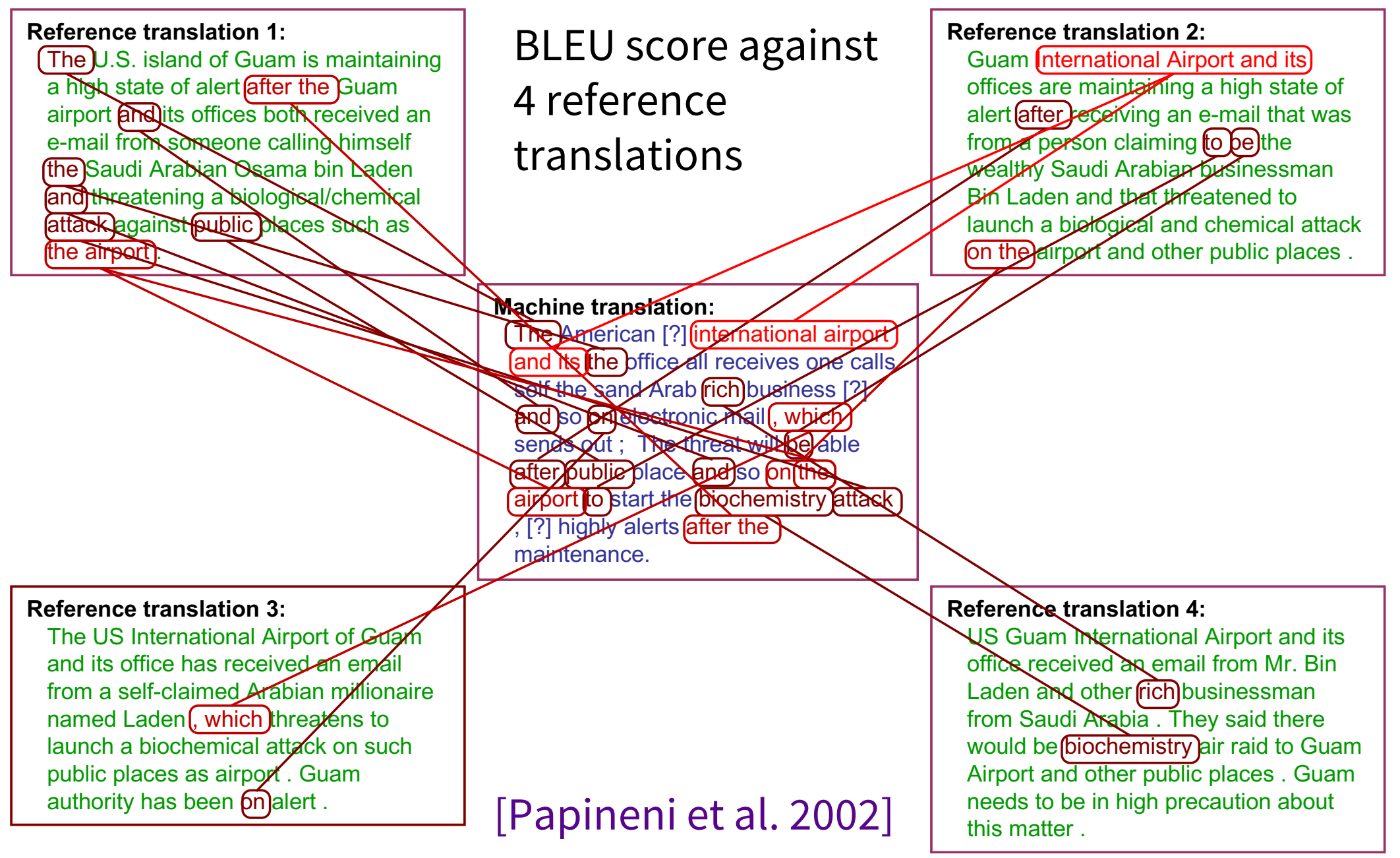




\section{Phrase-Based Statistical MT:}

\section{Pharaoh/Moses}

[Koehn et al, 2003]

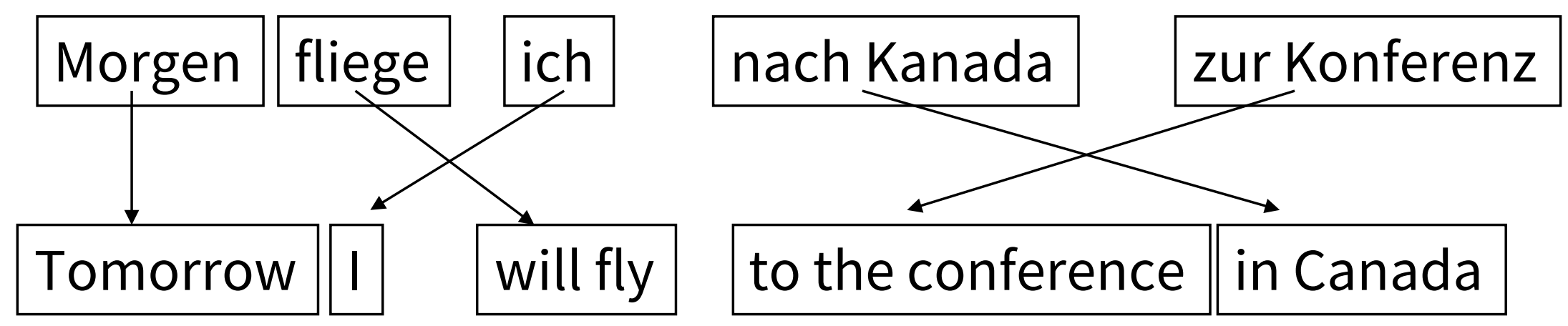

Source input segmented into phrases

- "phrase" is a subsequence of words - not linguistic phrase

$\rightarrow$ Do we need phrases in NMT?

Or not, as have in-context word translation?

Cf. [Kalchbrenner \& Blunsom 2013] source CNN and [Eriguchi, Hashimoto \& Tsuruoka 2016] source tree 


\section{SMT phrase table weights gave}

\section{a context-independent translation score}

\section{Each phrase is probabilistically translated}

- P(in spite| 尽管)

- P(in spite of the fact $\mid$ 尽管)

开发 ||| development ||| (0) ||| (0) ||| -2.97 -2.72 -0.86 -0.95

开发 || development of ||| (0) ||| (0) () ||| -3.41 -2.72 -3.22 -3.50

进行 监督 ||| that carries out a supervisory ||| (1,2,3) (4) ||| () (0) (0) (0) (1) ||| 0.0 -3.68 -7.27 -21.24

进行 监督 ||| carries out a supervisory ||| (0,1,2) (3) ||| (0) (0) (0) (1) ||| 0.0 -3.68 -7.27-17.17

监督 ||| supervisory ||| (0) ||| (0) ||| -1.03-0.80-3.68 -3.24

监督检查 || supervisory inspection ||| (0) (1) ||| (0) (1) ||| 0.0 -2.33 -6.07-4.

检查 ||| inspection ||| (0) ||| (0) ||| -1.54-1.53-2.05-1.60

尽管 ||| in spite ||| (1) ||| () (0) ||| -0.90-0.50 -3.56 -6.14

尽管 ||| in spite of ||| (1) ||| () (0) () ||| -1.11 -0.50 -3.93 -8.68

尽管 ||| in spite of the ||| (1) ||| () (0) () () ||| -1.06 -0.50 -4.77 -10.50

尽管 ||| in spite of the fact ||| (1) ||| () (0) () () () ||| -1.18 -0.50 -6.54 -18.19 


\section{Phrase-based SMT:}

Log-linear feature-based MT models

$\hat{e}=\operatorname{argmax}_{e} 1.9 \times \log \mathrm{P}(e)+1.0 \times \log \mathrm{P}(f \mid e)+1.1 \times$ log length $(e)+\ldots$

$=\operatorname{argmax}_{e} \Sigma_{i} w_{i} \phi_{i}$

We have two things:

- "Features" $\phi$, such as log translation model score - Weights $w$ for each feature for how good it is

The weights were learned

Feature scores from separately trained models 
Language Models (LM)

A language model - $P(e)$ - gives the probability of a sequence of words

Most important feature! Why not just do more with language models?

Egg., generate a translation with LM also conditioned on source $\rightarrow$ Use NLM 34 


\section{MT Decoder: Beam Search}

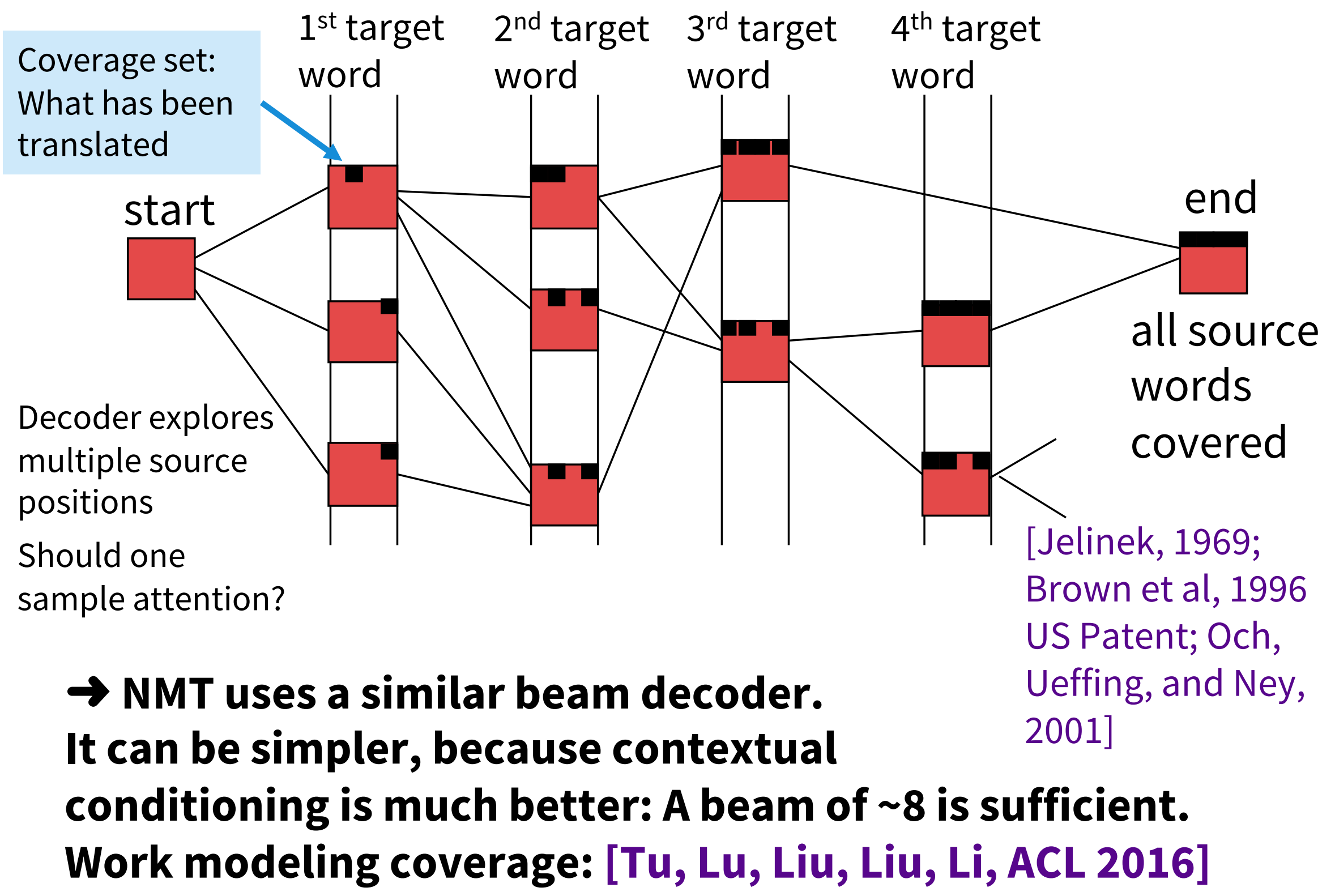


An NMT system is an NLM with extra conditioning! 1c. Neural Language Models 


\section{Language Models: Sentence probabilities}

$p\left(x_{1}, x_{2}, \ldots, x_{T}\right)=\prod_{t=1}^{T} p\left(x_{t} \mid x_{1}, \ldots, x_{t-1}\right)$

[Chain rule]

The

There are way too many histories once you're into a sentence a few words! Exponentially many. 


\section{Traditional Fix: Markov Assumption}

An $n^{\text {th }}$ order Markov assumption assumes each word depends only on a short linear history

$$
\begin{aligned}
p\left(x_{1}, x_{2}, \ldots, x_{T}\right) & =\prod_{t=1}^{T} p\left(x_{t} \mid x_{1}, \ldots, x_{t-1}\right) \\
& \approx \prod_{t=1}^{T} p\left(x_{t} \mid x_{t-n}, \ldots, x_{t-1}\right)
\end{aligned}
$$




\section{Problems of Traditional Markov \\ Model Assumptions (1): Sparsity}

Issue: Very small window gives bad prediction; statistics for even a modest window are sparse

\section{Example:}

$$
\mathrm{P}\left(w_{0} \mid w_{-3}, w_{-2}, w_{-1}\right) \quad|V|=100,000 \rightarrow 10^{15} \text { contexts }
$$

Most have not been seen

The traditional answer is to use various backoff and smoothing techniques, but no good solution 
Neural Language Models

The neural approach [Bengio, Ducharme, vincent \& JaUvin JMLR 2003] represents words as dense distributed vectors so there can be sharing of statistical weight between similar words

Doing just this solves the sparseness problem of conventional $n$-gram models 


\section{Neural (Probabilistic) Language Model [Bengio, Ducharme, Vincent \& Jauvin JMLR 2003]}

$w_{-3}$
registration
$w_{-2}$
is
$w_{-7}$
now


Neural (Probabilistic) Language Model [Bengio, Ducharme, Vincent \& Jauvin JMLR 2003]

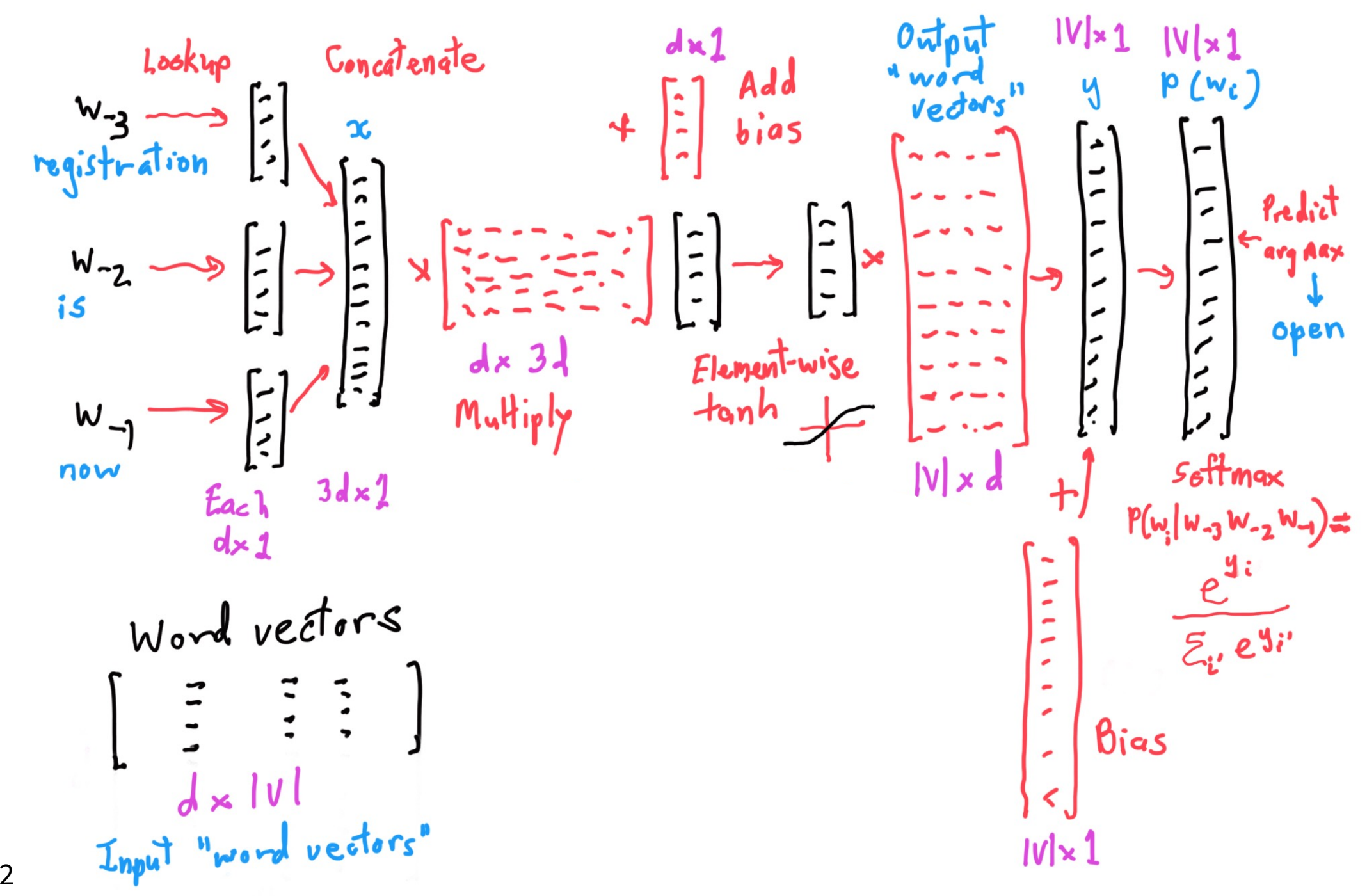


Neural (Probabilistic) Language Model [Bengio, Ducharme, Vincent \& Jauvin JMLR 2003]

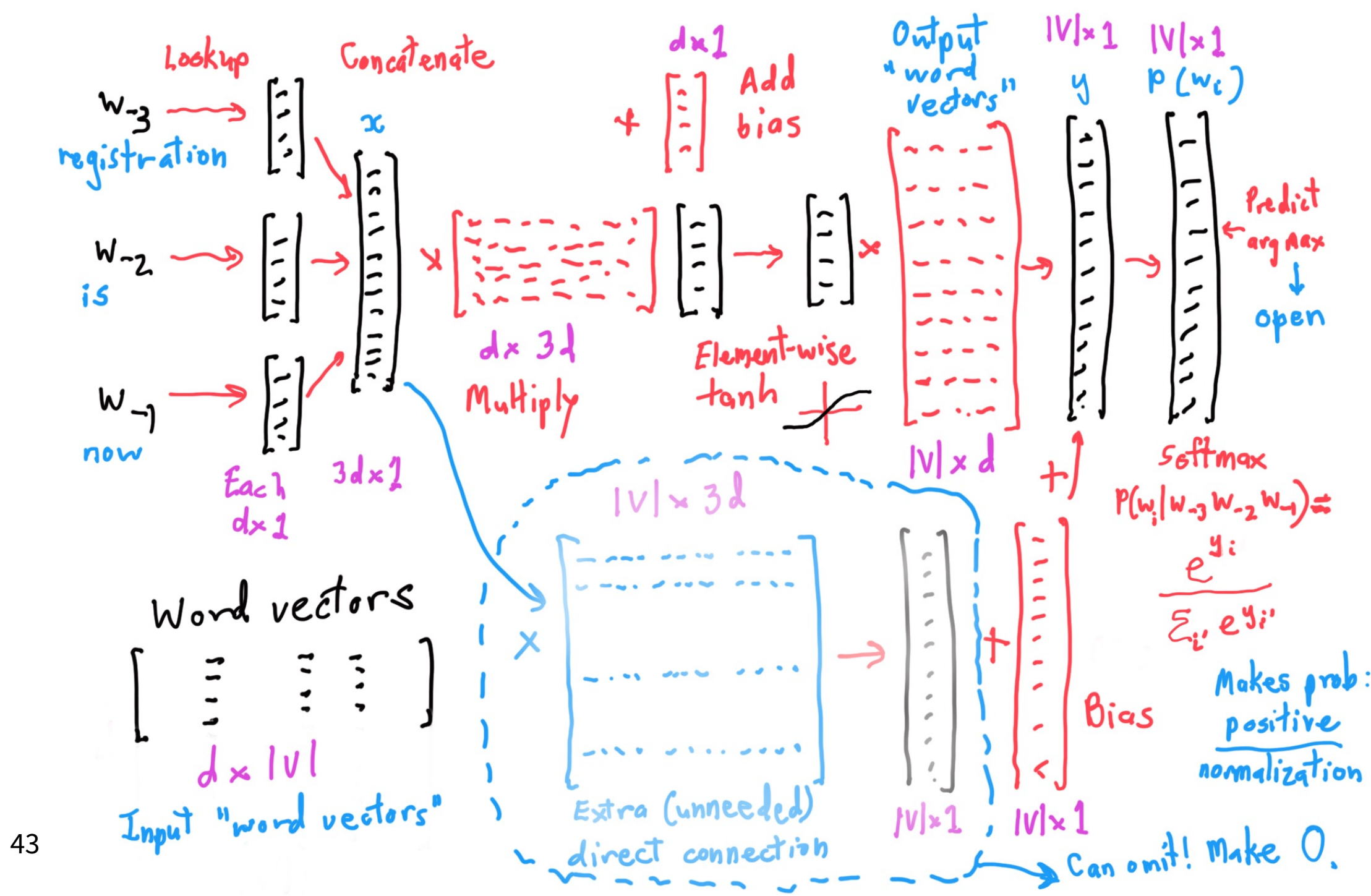




\section{Problems of Traditional Markov Model Assumptions (2): Context}

Issue: Dependency beyond the window is ignored

\section{Example:}

the same stump which had impaled the car of many a guest in the past thirty years and which he refused to have removed 


\section{A Non-Markovian Language Model}

Can we directly model the true conditional probability?

$$
p\left(x_{1}, x_{2}, \ldots, x_{T}\right)=\prod_{t=1}^{T} p\left(x_{t} \mid x_{1}, \ldots, x_{t-1}\right)
$$

Can we build a neural language model for this?

1. Feature extraction: $h_{t}=f\left(x_{1}, x_{2}, \ldots, x_{t}\right)$

2. Prediction: $p\left(x_{t+1} \mid x_{1}, \ldots, x_{t-1}\right)=g\left(h_{t}\right)$

How can $f$ take a variable-length input? 


\section{A Non-Markovian Language Model}

Can we directly model the true conditional probability?

$$
p\left(x_{1}, x_{2}, \ldots, x_{T}\right)=\prod_{t=1}^{T} p\left(x_{t} \mid x_{1}, \ldots, x_{t-1}\right)
$$

Recursive construction of $f$

1. Initialization $h_{0}=0$

2. Recursion $h_{t}=f\left(x_{t}, h_{t-1}\right)$

We call $h_{t}$ a hidden state or memory

$h_{t}$ summarizes the history $\left(x_{1}, \ldots, x_{t}\right)$

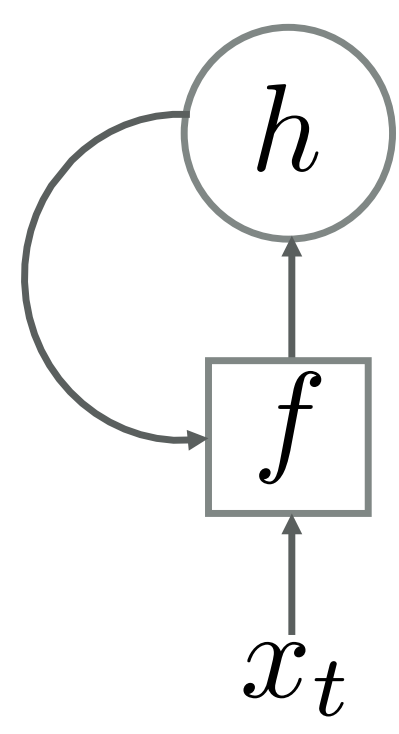




\section{A Non-Markovian Language Model}

Example: $\quad p$ (the, cat, is, eating)

(1) Initialization: $h_{0}=0$

(2) Recursion with Prediction:

$$
\begin{aligned}
& h_{1}=f\left(h_{0},\langle\text { bos }\rangle\right) \rightarrow p(\text { the })=g\left(h_{1}\right) \\
& h_{2}=f\left(h_{1}, \text { cat }\right) \rightarrow p(\text { cat } \mid \text { the })=g\left(h_{2}\right) \\
& h_{3}=f\left(h_{2}, \text { is }\right) \rightarrow p(\text { is } \mid \text { the }, \text { cat })=g\left(h_{3}\right) \\
& h_{4}=f\left(h_{3}, \text { eating }\right) \rightarrow p(\text { eating } \mid \text { the }, \text { cat }, \text { is })=g\left(h_{4}\right)
\end{aligned}
$$

(3) Combination:

$$
p(\text { the }, \text { cat }, \text { is, eating })=g\left(h_{1}\right) g\left(h_{2}\right) g\left(h_{3}\right) g\left(h_{4}\right)
$$

47 Read, Update and Predict 


\section{A Recurrent Neural Network Language Model solves the second problem!}

Example: $p$ (the, cat, is, eating)

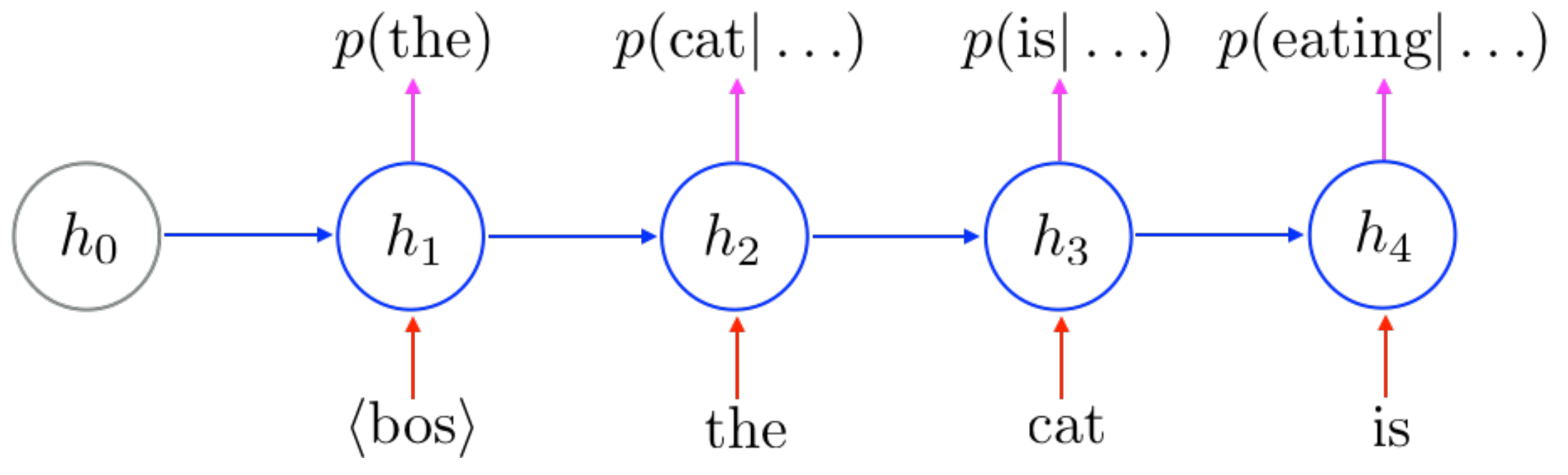

Read, Update and Predict 


\section{Building a Recurrent Language Model}

Transition Function $h_{t}=f\left(h_{t-1}, x_{t}\right)$

Inputs

i. Current word $x_{t} \in\{1,2, \ldots,|V|\}$

ii. Previous state $h_{t-1} \in \mathbb{R}^{d}$

Parameters

i. Input weight matrix $W \in \mathbb{R}^{|V| \times d}$

ii. Transition weight matrix $U \in \mathbb{R}^{d \times d}$

iii. Bias vector $b \in \mathbb{R}^{d}$

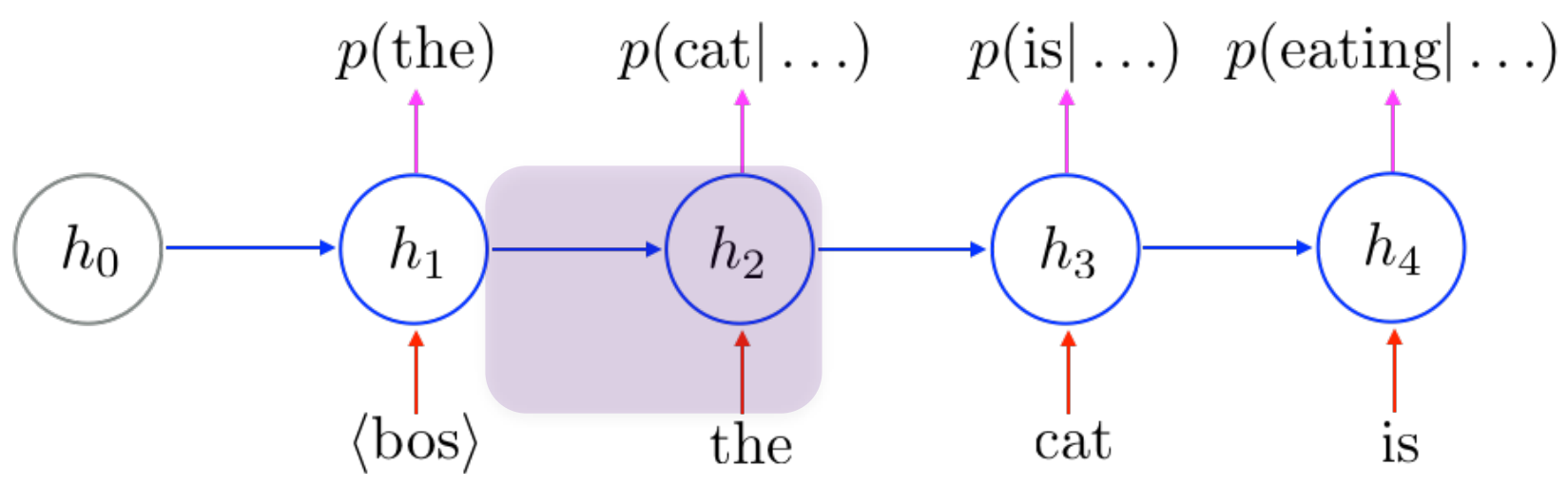




\section{Building a Recurrent Language Model}

Transition Function $h_{t}=f\left(h_{t-1}, x_{t}\right)$

Naïve Transition Function

$$
f\left(h_{t-1}, x_{t}\right)=\tanh \left(W\left[x_{t}\right]+U h_{t-1}+b\right)
$$

Element-wise nonlinear transformation

\section{Trainable word vector}

\section{Linear transformation of previous state}

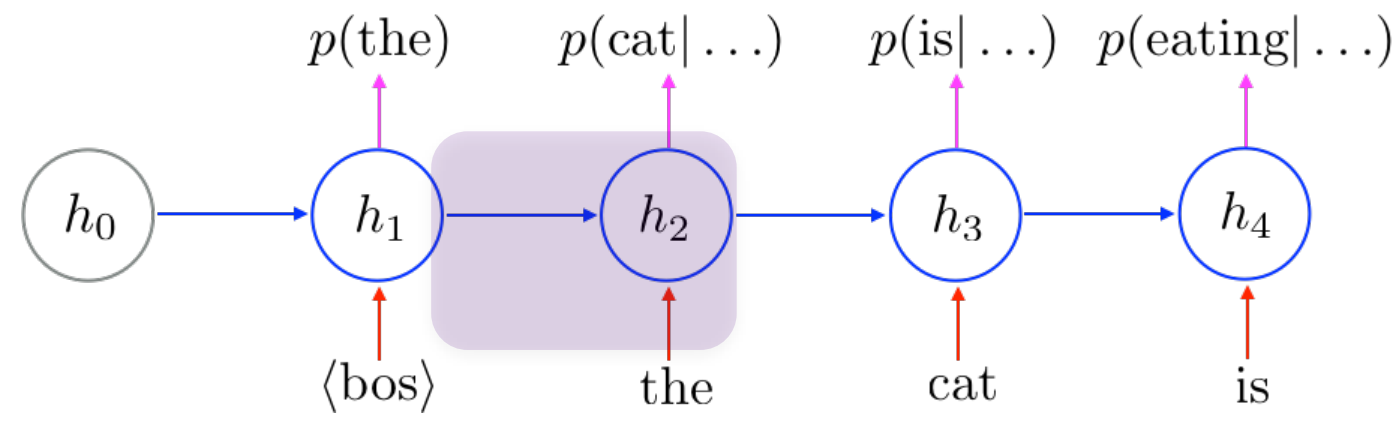




\section{Building a Recurrent Language Model}

Prediction Function $p\left(x_{t+1}=w \mid x_{\leq t}\right)=g_{w}\left(h_{t}\right)$

Inputs

i. Current state $h_{t} \in \mathbb{R}^{d}$

Parameters

i. Softmax matrix $R \in \mathbb{R}^{|V| \times d}$

ii. Bias vector $c \in \mathbb{R}^{|V|}$

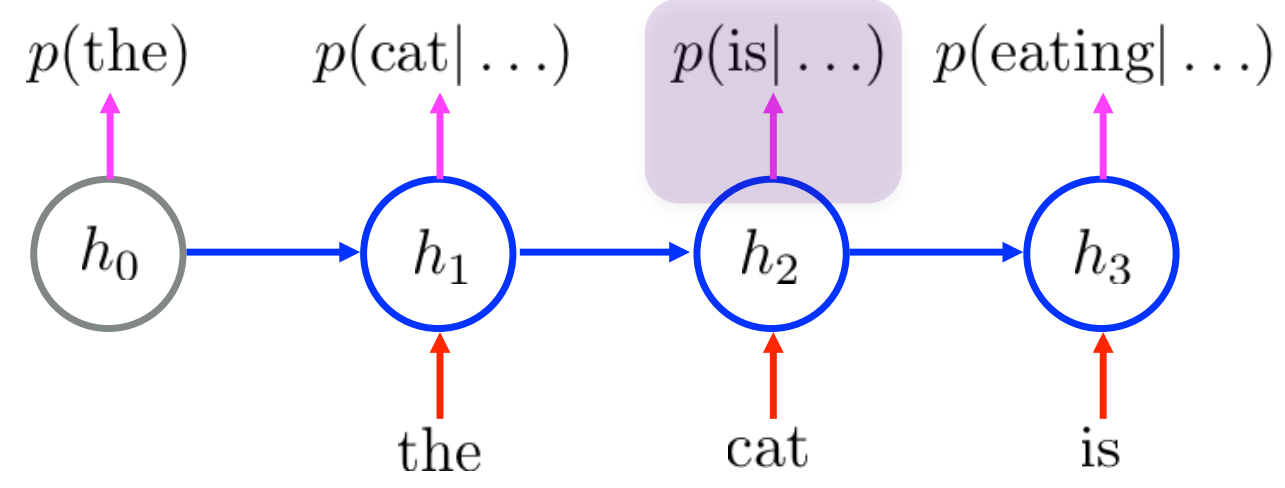




\section{Building a Recurrent Language Model}

Prediction Function $p\left(x_{t+1}=w \mid x_{\leq t}\right)=g_{w}\left(h_{t}\right)$
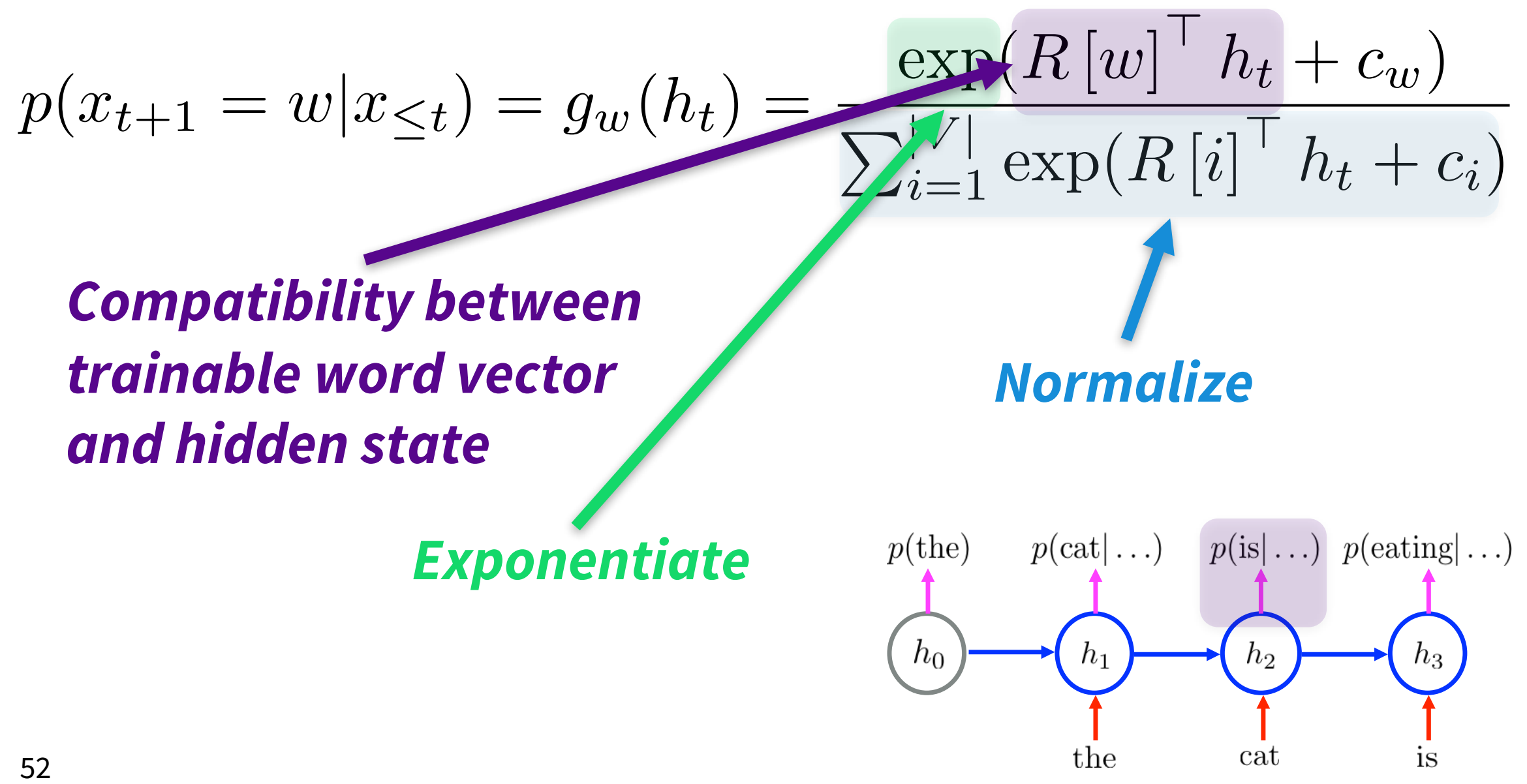


\section{Training a recurrent language model}

Having determined the model form, we:

1. Initialize all parameters of the models, including the word representations with small random numbers

2. Define a loss function: how badly we predict actual next words [log loss or cross-entropy loss]

3. Repeatedly attempt to predict each next word

4. Backpropagate our loss to update all parameters

5. Just doing this learns good word representations ${ }_{53}$ and good prediction functions - it's almost magic 


\section{Neural Language Models as MT components}

You can just replace the target-side language model of a conventional phrase-based SMT system with an NLM

NLM / Continuous space language models

[Schwenk, Costa-Jussà \& Fonollosa 2006; Schwenk 2007; Auli \& Gao 2013; Vaswani, Zhao, Fossum \& Chiang 2013]

You can use the source as well as target words to predict next target word, usually using phrase alignment

Neural Joint Language Models

[Auli, Galley, Quirk \& Zweig 2013; Devlin, Zbib, Huang, Lamar, Schwartz \& Makhool 2014] 


\section{However,}

we want to move on to the

goal of an end-to-end trained neural translation model! 


\section{Recurrent Language Model}

Example) $p$ (the, cat, is, eating)

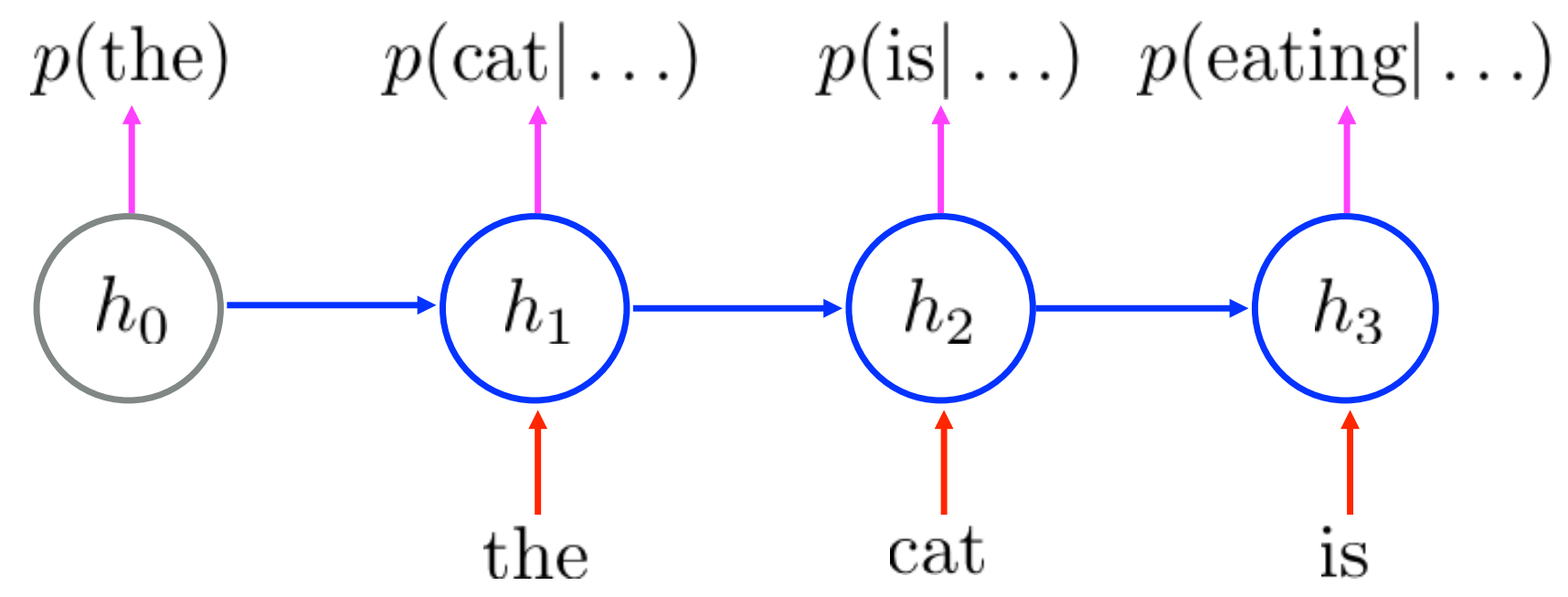

Read, Update and Predict 


\section{2a. Training a Recurrent Language Model}

Maximum likelihood estimation with stochastic gradient descent and backpropagation through time 


\section{Training a Recurrent Language Model}

- Log-probability of one training sentence

$$
\log p\left(x_{1}^{n}, x_{2}^{n}, \ldots, x_{T^{n}}^{n}\right)=\sum_{t=1}^{T^{n}} \log p\left(x_{t}^{n} \mid x_{1}^{n}, \ldots, x_{t-1}^{n}\right)
$$

- $\quad$ Training set $D=\left\{X^{1}, X^{2}, \ldots, X^{N}\right\}$

- Log-likelihood Functional

$$
\mathcal{L}(\theta, D)=\frac{1}{N} \sum_{n=1}^{N} \sum_{t=1}^{T^{n}} \log p\left(x_{t}^{n} \mid x_{1}^{n}, \ldots, x_{t-1}^{n}\right)
$$

Minimize $-\mathcal{L}(\theta, D) ! !$

$p$ (the) $\quad p($ cat $\mid \ldots) \quad p($ is $\mid \ldots) \quad p($ eating $\mid \ldots)$

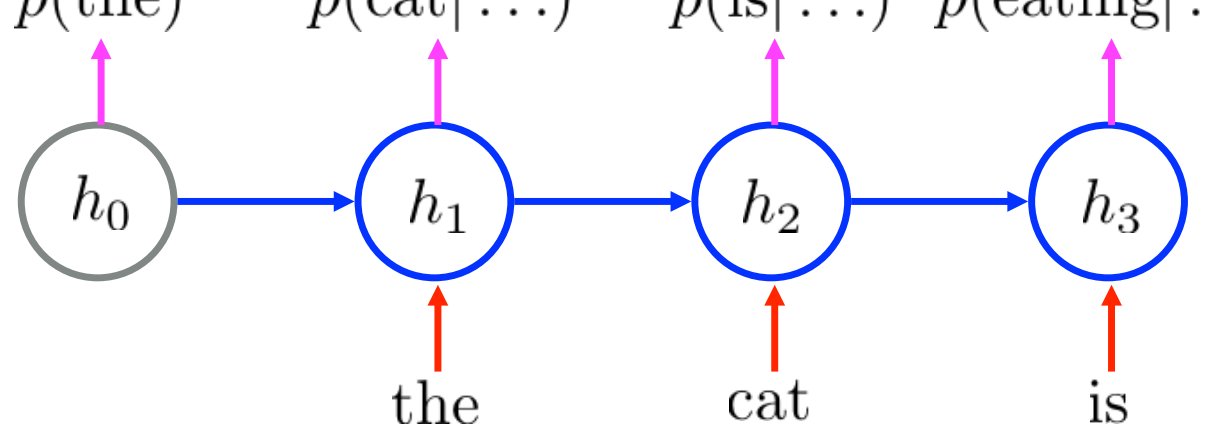




\section{Gradient Descent}

- Move slowly in the steepest descent direction

$$
\theta \leftarrow \theta-\eta \nabla \mathcal{L}(\theta, D)
$$

- Computational cost of a single update: $O(N)$

- Not suitable for a large corpus
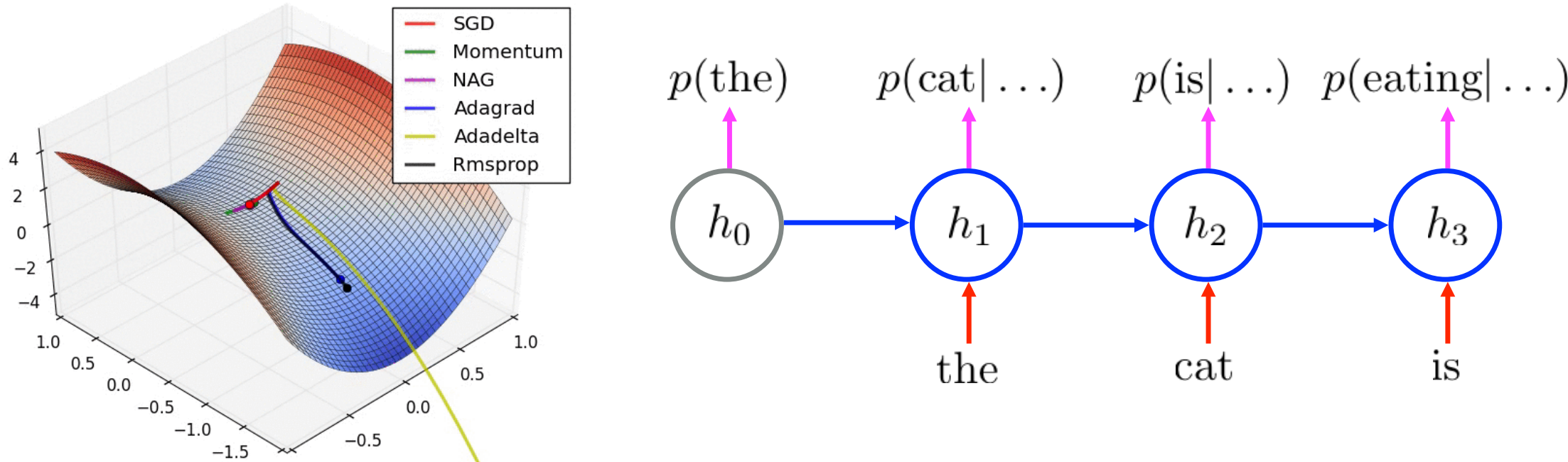


\section{Stochastic Gradient Descent}

- Estimate the steepest direction with a minibatch

$$
\nabla \mathcal{L}(\theta, D) \approx \nabla \mathcal{L}\left(\theta,\left\{X^{1}, \ldots, X^{n}\right\}\right)
$$

- Until the convergence (w.r.t. a validation set)

$$
\left|\mathcal{L}\left(\theta, D_{\text {val }}\right)-\mathcal{L}\left(\theta-\eta \mathcal{L}(\theta, D), D_{\text {val }}\right)\right| \leq \epsilon
$$
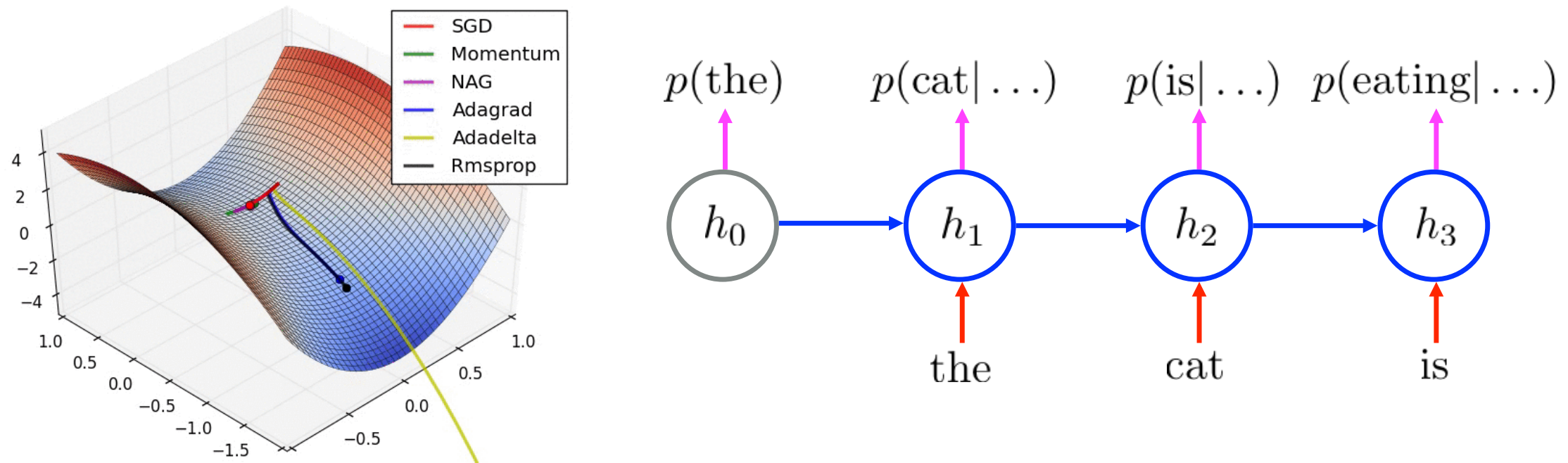


\section{Stochastic Gradient Descent}

- Not trivial to build a minibatch

\begin{tabular}{l}
\hline \multicolumn{2}{|c|}{ Sentence 1 } \\
\hline Sentence 2 \\
\hline \multicolumn{2}{|c|}{ Sentence 3 } \\
\hline \multicolumn{2}{|c|}{ Sentence 4 } \\
Padding and Masking: suitable for GPU's, but wasteful \\
- Wasted computation \\
\begin{tabular}{|l|c}
\hline \multicolumn{2}{|c|}{ 0's } \\
\hline Sentence 2 & O's \\
\hline \multicolumn{2}{|c|}{ Sentence 3 } \\
\hline Sentence 4 & 0's \\
\hline
\end{tabular}
\end{tabular}




\section{Stochastic Gradient Descent}

1. Padding and Masking: suitable for GPU's, but wasteful

- Wasted computation

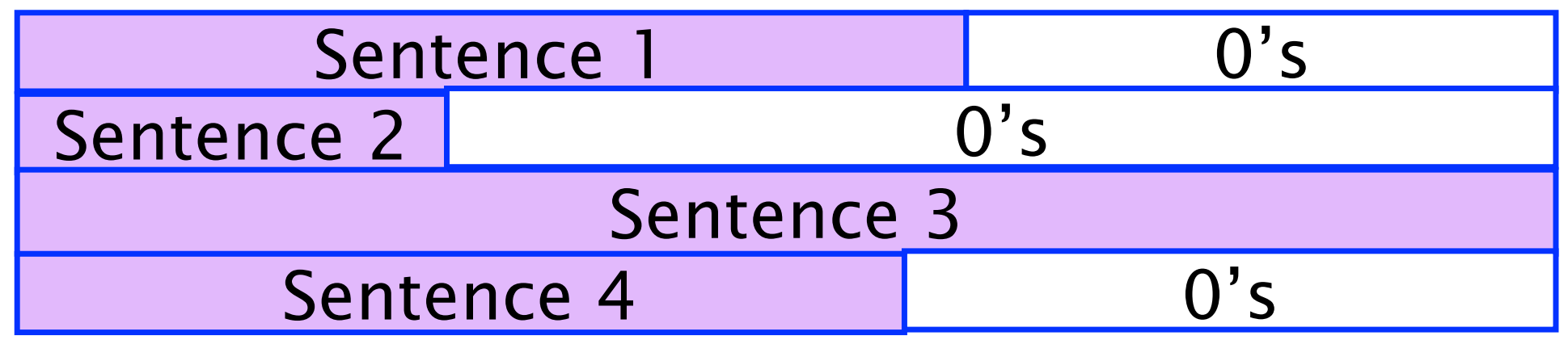

2. Smarter Padding and Masking: minimize the waste

- Ensure that the length differences are minimal.

- Sort the sentences and sequentially build a minibatch

Sentence 1

Sentence 2

Sentence 3

62

\begin{tabular}{|c|c|}
\hline Sentence 1 & \multicolumn{2}{c|}{ 0's } \\
\hline Sentence 2 & 0's \\
\hline Sentence 3 & 0's \\
\hline Sentence 4 \\
\hline
\end{tabular}




\section{Backpropagation through Time}

How do we compute $\nabla \mathcal{L}(\theta, D)$ ?

- Cost as a sum of per-sample cost function

$$
\nabla \mathcal{L}(\theta, D)=\sum_{X \in D} \nabla \mathcal{L}(\theta, X)
$$

- Per-sample cost as a sum of per-step cost functions

$$
\begin{aligned}
& \nabla \mathcal{L}(\theta, X)=\sum_{t=1}^{T} \nabla \log p\left(x_{t} \mid x_{<t}, \theta\right) \quad \log p\left(x_{t} \mid x_{<t}\right) \\
& \nabla \mathcal{L}(\theta, X)=\sum_{t=1} \nabla \log p\left(x_{t} \mid x_{<t}, \theta\right)
\end{aligned}
$$

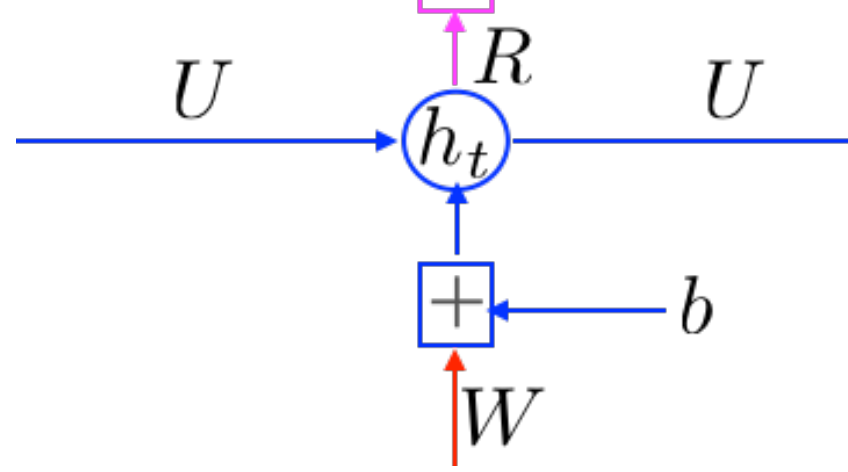




\section{Backpropagation through Time}

\section{How do we compute $\nabla \log p\left(x_{t} \mid x_{<t}, \theta\right)$ ?}

- Compute per-step cost function from time $t=T$

1. Cost derivative $\partial \log p\left(x_{t} \mid x_{<t}\right) / \partial g$

2. Gradient w.r.t. $R: \times \partial g / \partial R$

3. Gradient w.r.t. $h_{t}: \times \partial g / \partial h_{t}+\partial h_{t+1} / \partial h_{t}$

4. Gradient w.r.t. $U: \times \partial h_{t} / \partial U$

5. Gradient w.r.t. $b$ and $W$ : $\times \partial h_{t} / \partial b$ and $\times \partial h_{t} / \partial W$

6. Accumulate the gradient and $t \leftarrow t-1$

$$
\log p\left(x_{t} \mid x_{<t}\right)
$$
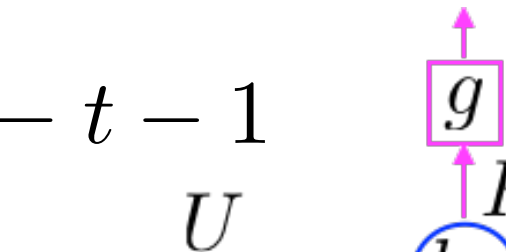


\section{Backpropagation through Time}

Intuitively, what's happening here?

1. Measure the influence of the past on the future

$\frac{\partial \log p\left(x_{t+n} \mid x_{<t+n}\right)}{\partial h_{t}}=\frac{\partial \log p\left(x_{t+n} \mid x_{<t+n}\right)}{\partial g} \frac{\partial g}{\partial h_{t+n}} \frac{\partial h_{t+n}}{\partial h_{t+n-1}} \cdots \frac{\partial h_{t+1}}{\partial h_{t}}$

2. How does the perturbation at $t$ affect $p\left(x_{t+n} \mid x_{<t+n}\right)$ ?

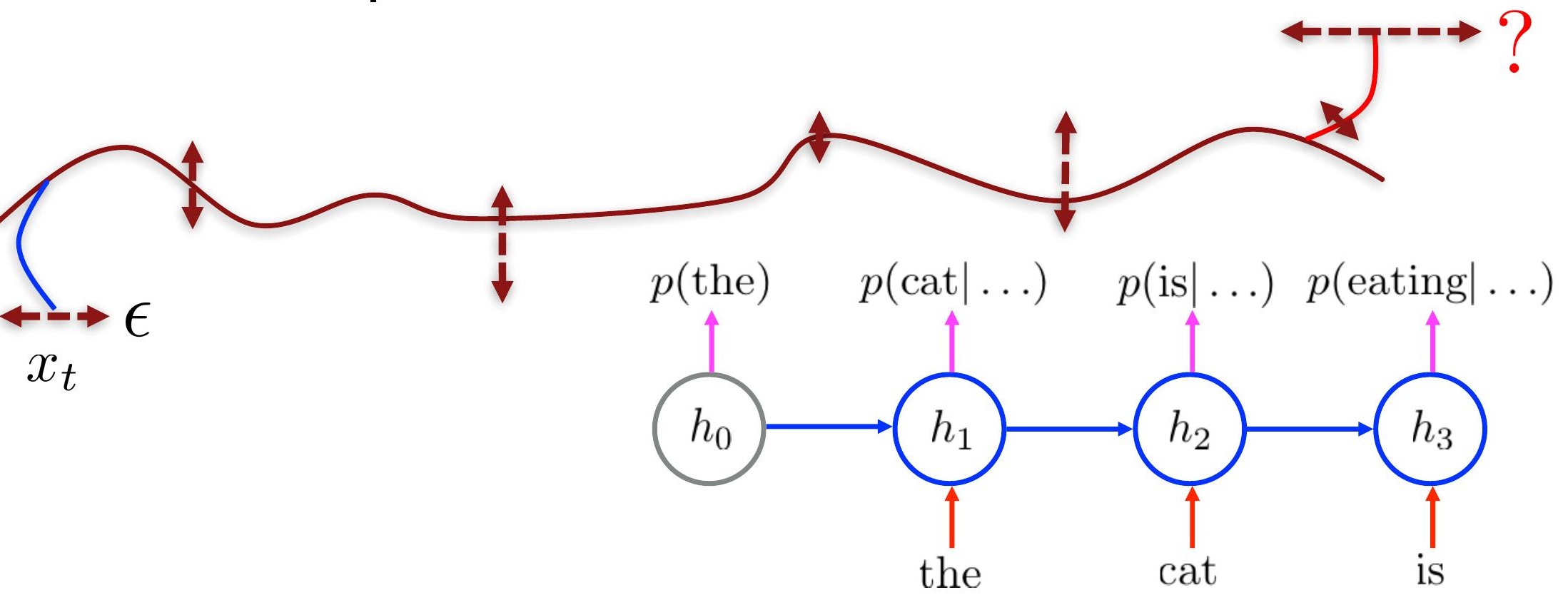




\section{Backpropagation through Time}

Intuitively, what's happening here?

1. Measure the influence of the past on the future

$\frac{\partial \log p\left(x_{t+n} \mid x_{<t+n}\right)}{\partial h_{t}}=\frac{\partial \log p\left(x_{t+n} \mid x_{<t+n}\right)}{\partial g} \frac{\partial g}{\partial h_{t+n}} \frac{\partial h_{t+n}}{\partial h_{t+n-1}} \cdots \frac{\partial h_{t+1}}{\partial h_{t}}$

2. How does the perturbation at $t$ affect $p\left(x_{t+n} \mid x_{<t+n}\right)$ ?

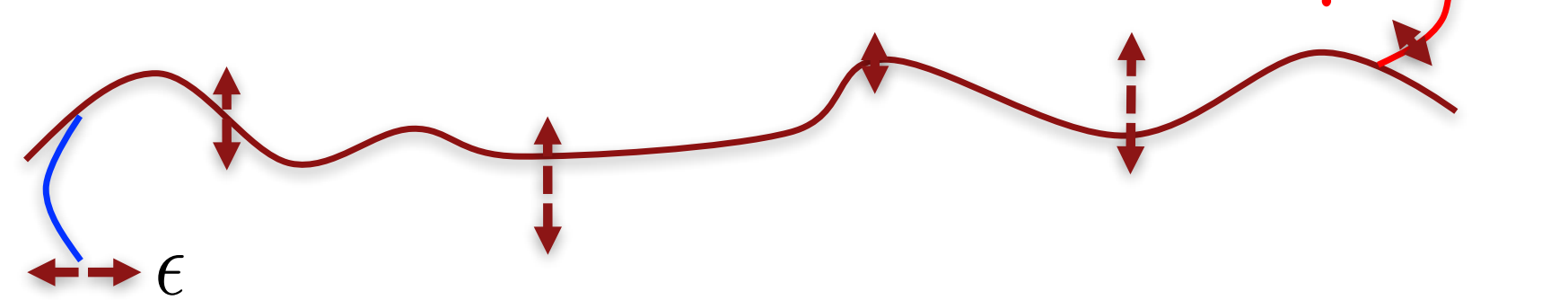

3. Change the parameters to maximize $p\left(x_{t+n} \mid x_{<t+n}\right)$ 


\section{Backpropagation through Time}

Intuitively, what's happening here?

1. Measure the influence of the past on the future

$\frac{\partial \log p\left(x_{t+n} \mid x_{<t+n}\right)}{\partial h_{t}}=\frac{\partial \log p\left(x_{t+n} \mid x_{<t+n}\right)}{\partial g} \frac{\partial g}{\partial h_{t+n}} \frac{\partial h_{t+n}}{\partial h_{t+n-1}} \cdots \frac{\partial h_{t+1}}{\partial h_{t}}$

2. With a naïve transition function

$$
\begin{gathered}
f\left(h_{t-1}, x_{t-1}\right)=\tanh \left(W\left[x_{t-1}\right]+U h_{t-1}+b\right) \\
\text { We get } \frac{\partial J_{t+n}}{\partial h_{t}}=\frac{\partial J_{t+n}}{\partial g} \frac{\partial g}{\partial h_{t+N}} \underbrace{\prod_{n=1}^{N} U^{\top} \operatorname{diag}\left(\frac{\partial \tanh \left(a_{t+n}\right)}{\partial a_{t+n}}\right)}_{\text {Problematic! }}
\end{gathered}
$$




\section{Backpropagation through Time}

\section{Gradient either vanishes or explodes}

- What happens?

$$
\frac{\partial J_{t+n}}{\partial h_{t}}=\frac{\partial J_{t+n}}{\partial g} \frac{\partial g}{\partial h_{t+N}} \underbrace{\prod_{n=1}^{N} U^{\top} \operatorname{diag}\left(\frac{\partial \tanh \left(a_{t+n}\right)}{\partial a_{t+n}}\right)}
$$

1. The gradient likely explodes if

$$
e_{\max } \geq \frac{1}{\max \tanh ^{\prime}(x)}=1
$$

2. The gradient likely vanishes if

$$
e_{\max }<\frac{1}{\max _{\tanh ^{\prime}(x)}}=1 \text {, where } e_{\max } \text { : largest eigenvalue of } U
$$




\section{Backpropagation through Time}

Addressing Exploding Gradient

- "when gradients explode so does the curvature along $v$, leading to a wall in the error surface"

- Gradient Clipping

1. Norm clipping

$$
\tilde{\nabla} \leftarrow \begin{cases}\frac{c}{\|\nabla\|} \nabla & \text {,if }\|\nabla\| \geq c \\ \nabla & \text {,otherwise }\end{cases}
$$

2. Element-wise clipping

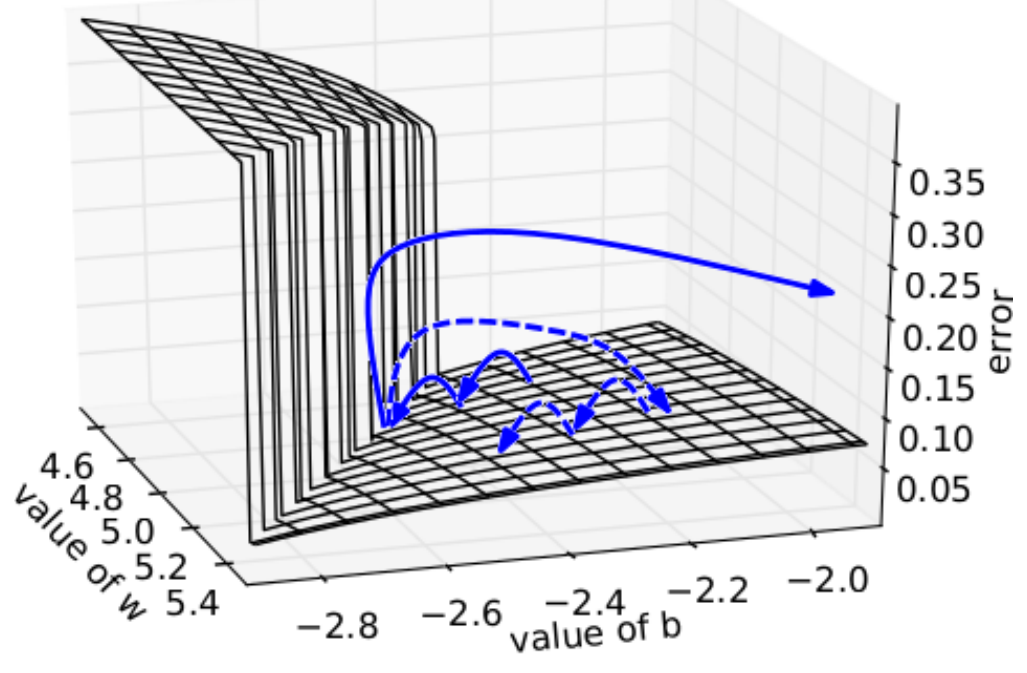

$$
\nabla_{i} \leftarrow \min \left(c,\left|\nabla_{i}\right|\right) \operatorname{sgn}\left(\nabla_{i}\right), \text { for all } i \in\{1, \ldots, \operatorname{dim} \nabla\}
$$




\section{Backpropagation through Time}

Vanishing gradient is super-problematic

- When we only observe

$$
\left\|\frac{\partial h_{t+N}}{\partial h_{t}}\right\|=\left\|\prod_{n=1}^{N} U^{\top} \operatorname{diag}\left(\frac{\partial \tanh \left(a_{t+n}\right)}{\partial a_{t+n}}\right)\right\| \rightarrow 0,
$$

- We cannot tell whether

1. No dependency between $t$ and $t+n$ in data, or

2. Wrong configuration of parameters:

$$
e_{\max }(U)<\frac{1}{\max \tanh ^{\prime}(x)}
$$




\section{2b. Gated Recurrent Units}

Vanishing gradient, gated recurrent units and long shortterm memory units 


\section{Gated Recurrent Unit}

- Is the problem with the naïve transition function?

$$
f\left(h_{t-1}, x_{t}\right)=\tanh \left(W\left[x_{t}\right]+U h_{t-1}+b\right)
$$

- With it, the temporal derivative is

$$
\frac{\partial h_{t+1}}{\partial h_{t}}=U^{\top} \frac{\partial \tanh (a)}{\partial a}
$$

- It implies that the error must be backpropagated through all the intermediate nodes:

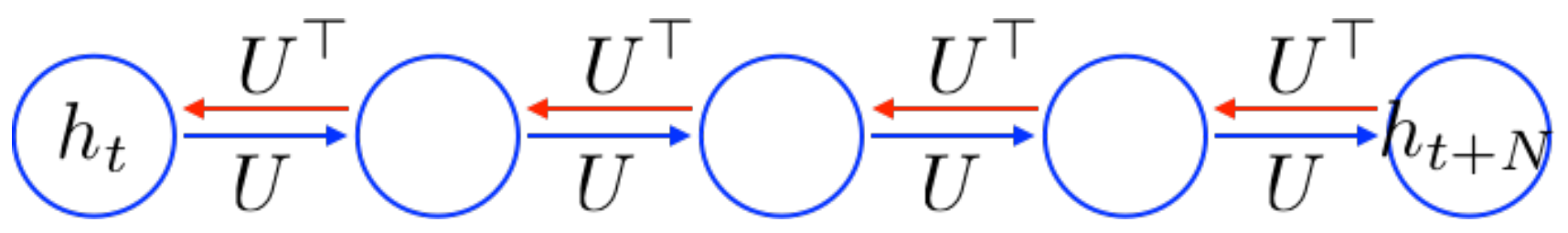




\section{Gated Recurrent Unit}

- It implies that the error must backpropagate through all the intermediate nodes:

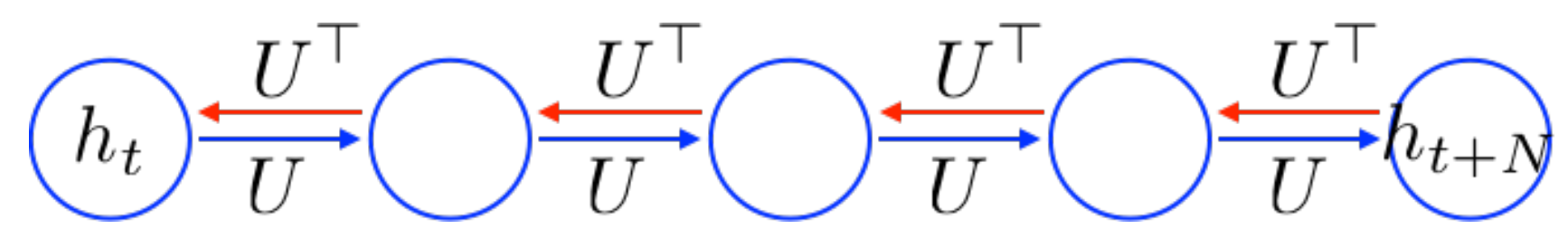

- Perhaps we can create shortcut connections.

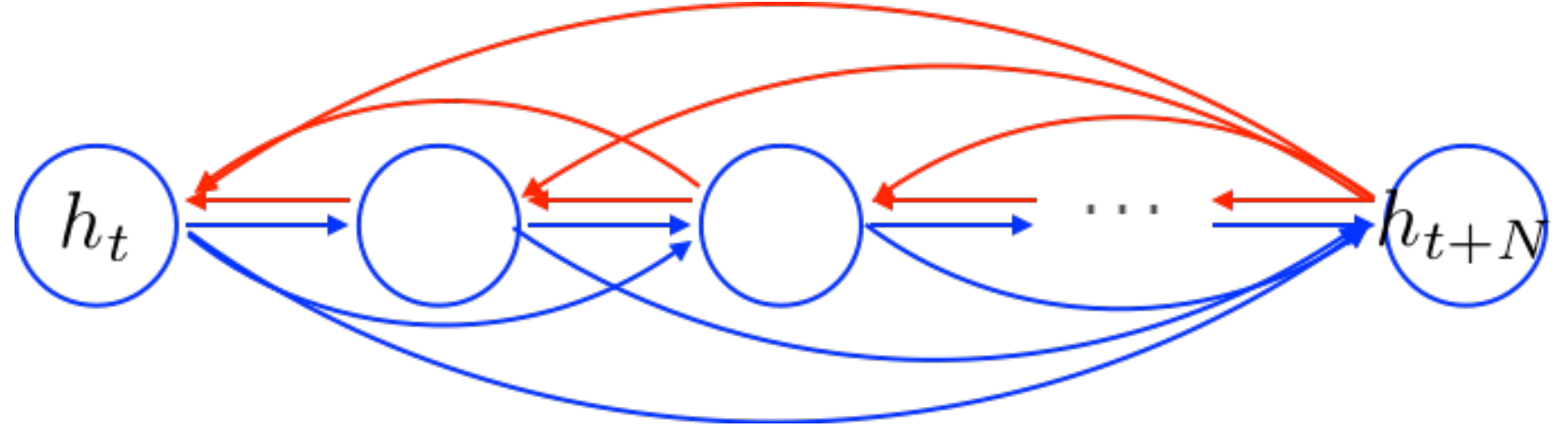




\section{Gated Recurrent Unit}

- Perhaps we can create adaptive shortcut connections.

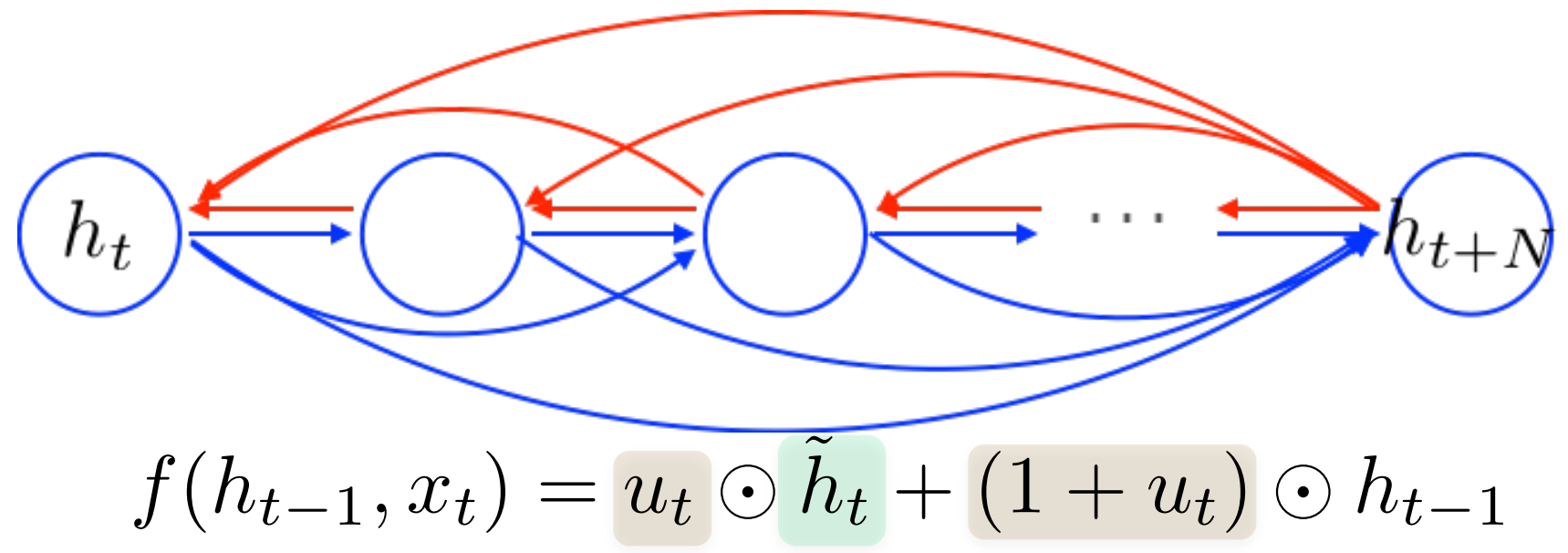

- Candidate Update $\tilde{h}_{t}=\tanh \left(W\left[x_{t}\right]+U h_{t-1}+b\right)$ Update gate $u_{t}=\sigma\left(W_{u}\left[x_{t}\right]+U_{u} h_{t-1}+b_{u}\right)$ 


\section{Gated Recurrent Unit}

- Let the net prune unnecessary connections adaptively.

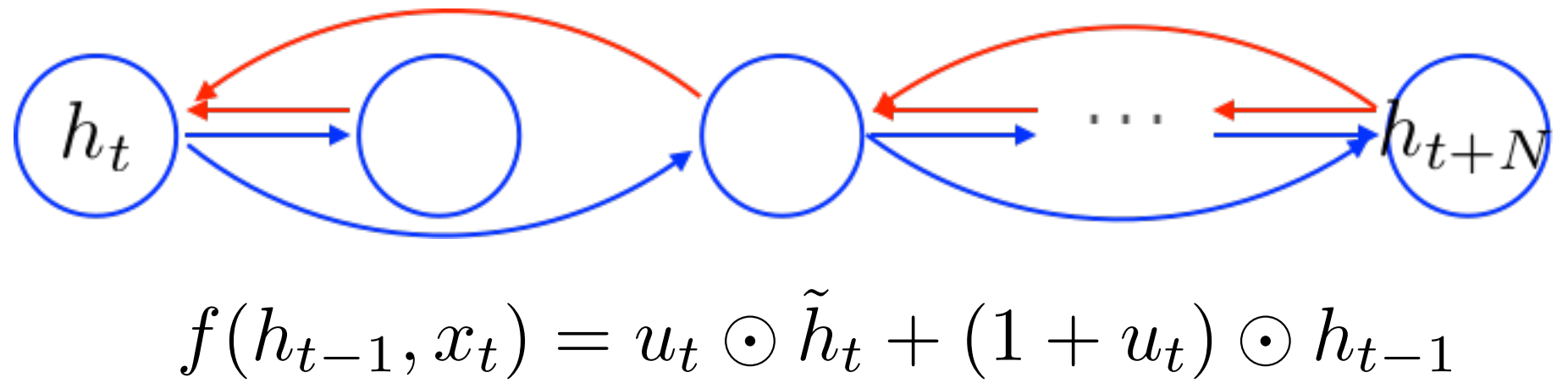

- Candidate Update $\tilde{h}_{t}=\tanh \left(W\left[x_{t}\right]+U\left(r_{t} \odot h_{t-1}\right)+b\right)$

- Reset gate $r_{t}=\sigma\left(W_{r}\left[x_{t}\right]+U_{r} h_{t-1}+b_{r}\right)$

- Update gate $u_{t}=\sigma\left(W_{u}\left[x_{t}\right]+U_{u} h_{t-1}+b_{u}\right)$ 


\section{Gated Recurrent Unit}

$\tanh -R N N \ldots$

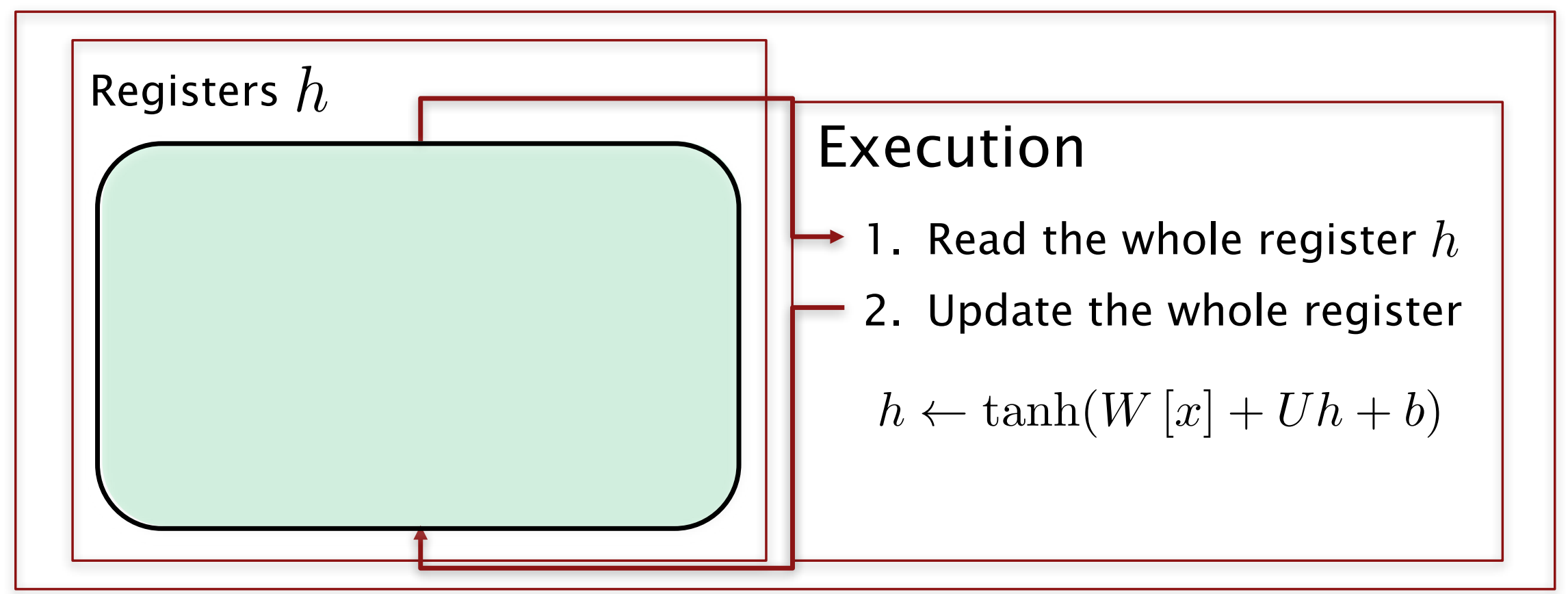




\section{Gated Recurrent Unit}

\section{GRU $\ldots$}

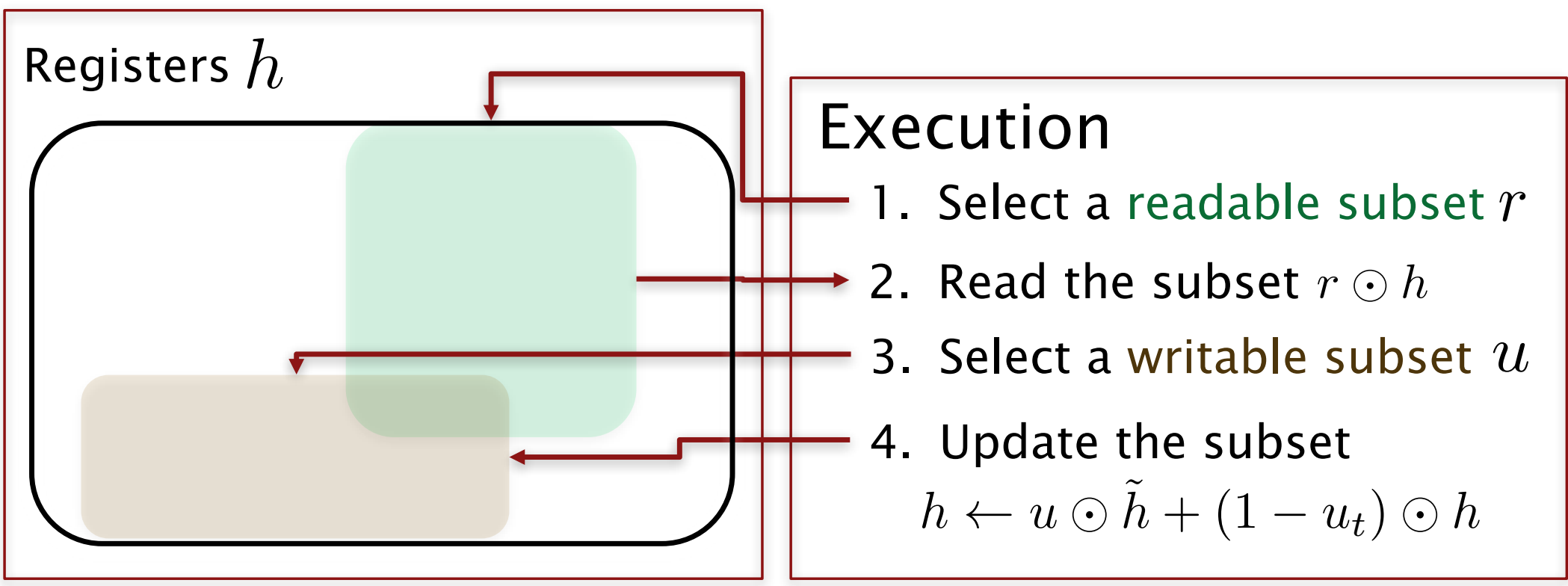

Clearly gated recurrent units are much more realistic. 


\section{Gated Recurrent Unit}

Two most widely used gated recurrent units

\section{Gated Recurrent Unit}

[Cho et al., EMNLP2014;

Chung, Gulcehre, Cho, Bengio, DLUFL2014]

$$
\begin{aligned}
& h_{t}=u_{t} \odot \tilde{h}_{t}+\left(1-u_{t}\right) \odot h_{t-1} \\
& \tilde{h}=\tanh \left(W\left[x_{t}\right]+U\left(r_{t} \odot h_{t-1}\right)+b\right) \\
& u_{t}=\sigma\left(W_{u}\left[x_{t}\right]+U_{u} h_{t-1}+b_{u}\right) \\
& r_{t}=\sigma\left(W_{r}\left[x_{t}\right]+U_{r} h_{t-1}+b_{r}\right)
\end{aligned}
$$

Long Short-Term Memory

[Hochreiter\&Schmidhuber, NC1999;

Gers, Thesis2001]

$$
\begin{aligned}
h_{t} & =o_{t} \odot \tanh \left(c_{t}\right) \\
c_{t} & =f_{t} \odot c_{t-1}+i_{t} \odot \tilde{c}_{t} \\
\tilde{c}_{t} & =\tanh \left(W_{c}\left[x_{t}\right]+U_{c} h_{t-1}+b_{c}\right) \\
o_{t} & =\sigma\left(W_{o}\left[x_{t}\right]+U_{o} h_{t-1}+b_{o}\right) \\
i_{t} & =\sigma\left(W_{i}\left[x_{t}\right]+U_{i} h_{t-1}+b_{i}\right) \\
f_{t} & =\sigma\left(W_{f}\left[x_{t}\right]+U_{f} h_{t-1}+b_{f}\right)
\end{aligned}
$$




\section{Training an RNN}

A few well-established + my personal wisdoms

1. Use LSTM or GRU: makes your life so much simpler

2. Initialize recurrent matrices to be orthogonal

3. Initialize other matrices with a sensible scale

4. Use adaptive learning rate algorithms: Adam, Adadelta, ...

5. Clip the norm of the gradient: " 1 " seems to be a reasonable threshold when used together with adam or adadelta.

6. Be patient! 


\title{
Now, go build and train a recurrent language model!
}

\author{
Any questions?
}


2C. Conditional Recurrent Language Model Encoder-Decoder Network for Machine Translation 


\section{Recurrent Language Model can}

1. Score a given sentence very well

$$
\log p \text { (the, cat, is, sitting, on, a, couch, .) }
$$

- Mere reranking significantly improves machine translation and speech recognition quality [Schwenk, 2007; Schwenk, 2012]

- Very good at sentence completion without much task-specific engineering [Tran, ..., Monz, NAACL 2016]

2. Generate a long, coherent text

- Observed earlier by Mikolov [2010, in his thesis] and Sutskever et al. [2011]

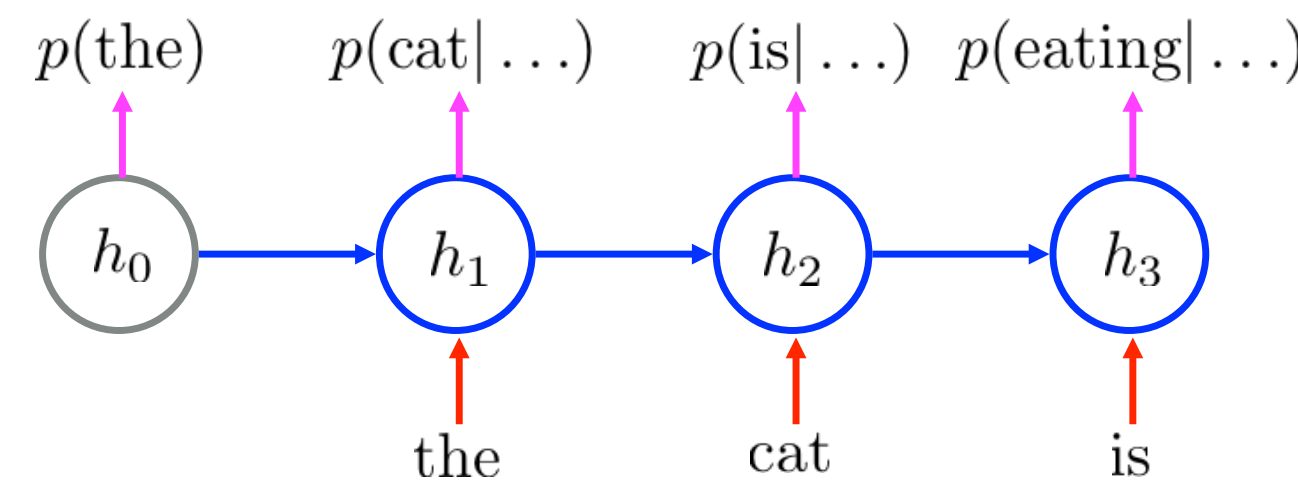




\section{Conditional Recurrent Language Model}

Le chat assis sur le tapis.

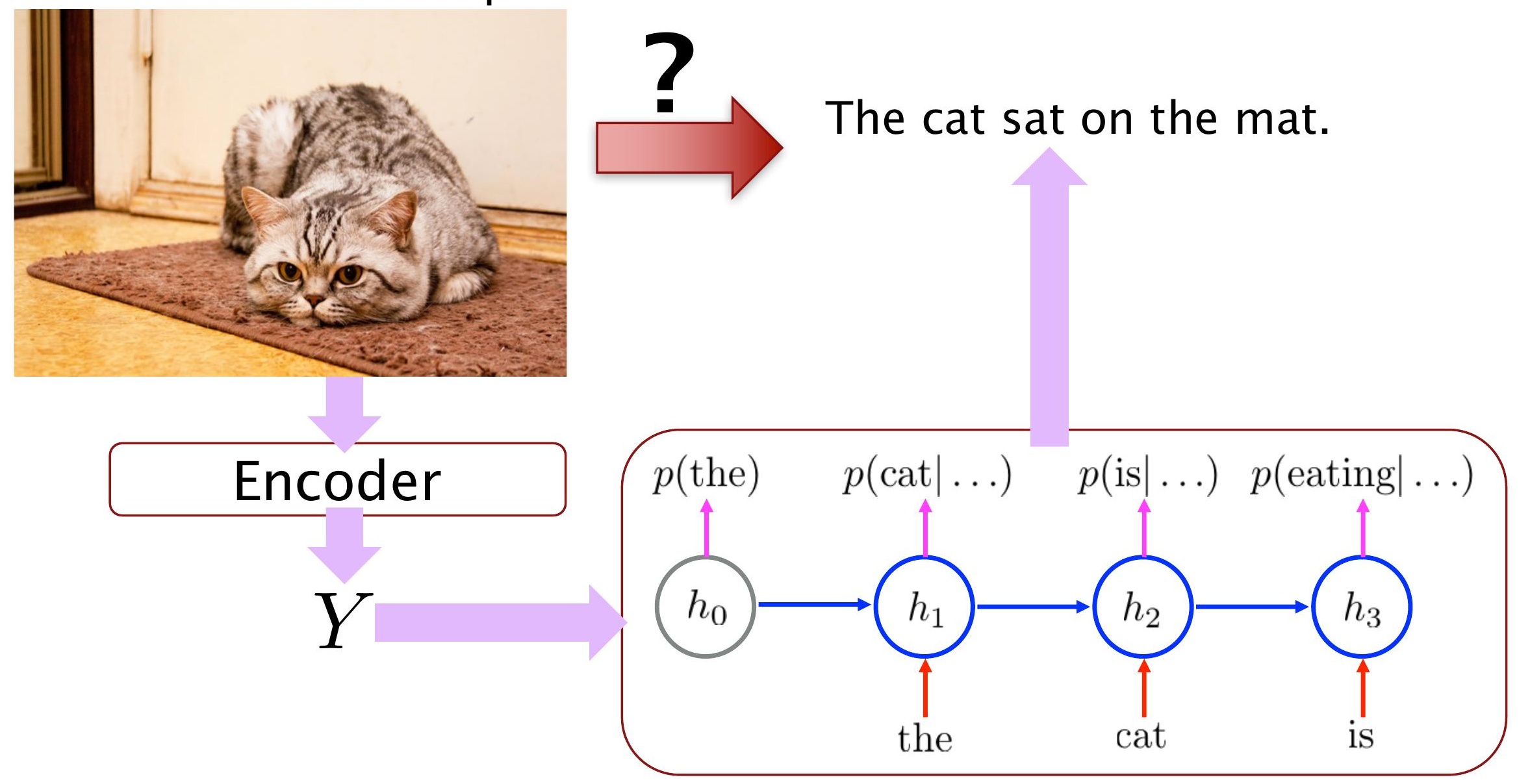




\section{Recurrent Neural Network Encoder}

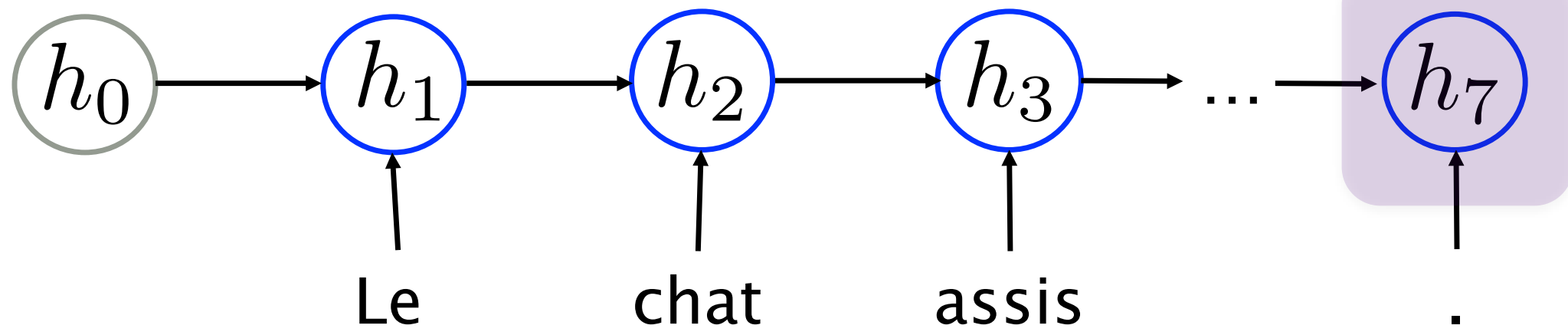

- Read a source sentence one symbol at a time.

- The last hidden state $Y$ summarizes the entire source sentence.

- Any recurrent activation function can be used:

- Hyperbolic tangent tanh

- Gated recurrent unit [Cho et al., 2014]

- Long short-term memory [Sutskever et al., 2014]

- Convolutional network [Kalchbrenner\&Blunsom, 2013] 


\section{Decoder: Recurrent Language Model}

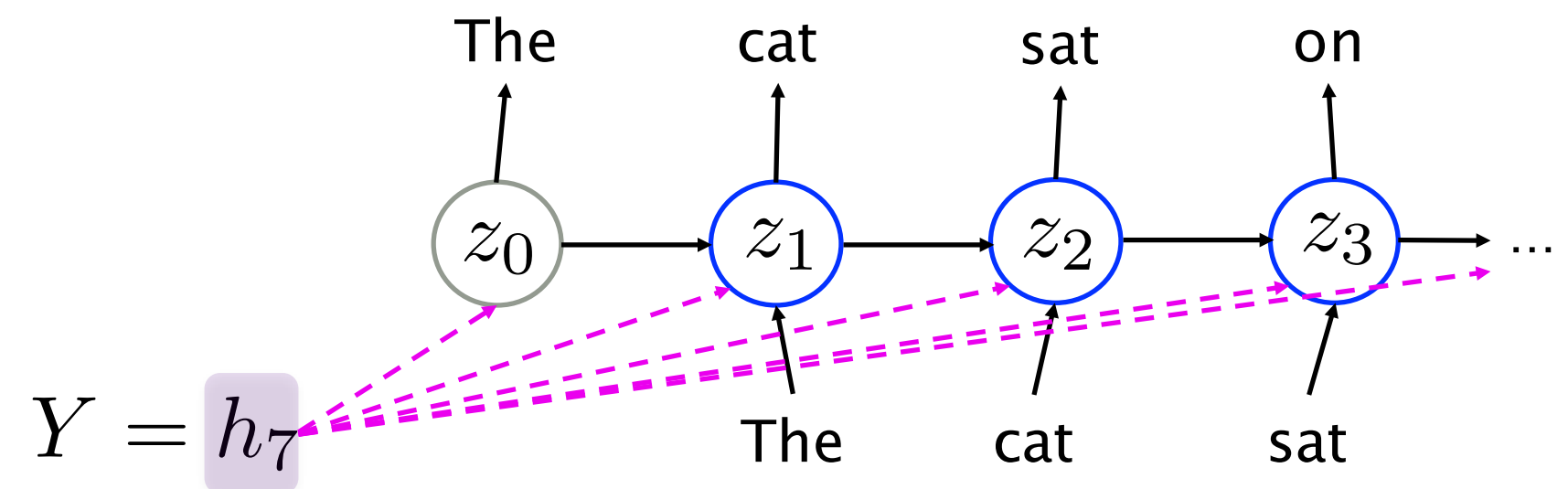

- Usual recurrent language model, except

1. Transition $z_{t}=f\left(z_{t-1}, x_{t}, Y\right)$

2. Backpropagation $\sum_{t} \partial z_{t} / \partial Y$

- Same learning strategy as usual: MLE with SGD

$$
\mathcal{L}(\theta, D)=\frac{1}{N} \sum_{n=1}^{N} \sum_{t=1}^{T^{n}} \log p\left(x_{t}^{n} \mid x_{1}^{n}, \ldots, x_{t-1}^{n}, Y\right)
$$




\section{With conditional recurrent language model,}

1. Score a translation

$$
\begin{aligned}
& \log p(\text { the, cat, is, sitting, on, a, couch, .| } \\
& \text { le, chat, est, assis, sur, un, canapé, .) }=?
\end{aligned}
$$

\section{Directly generate a translation}

le,chat, est, assis, sur, un, canapé, .

$\mapsto$ the, cat, is, sitting, on, a, couch,

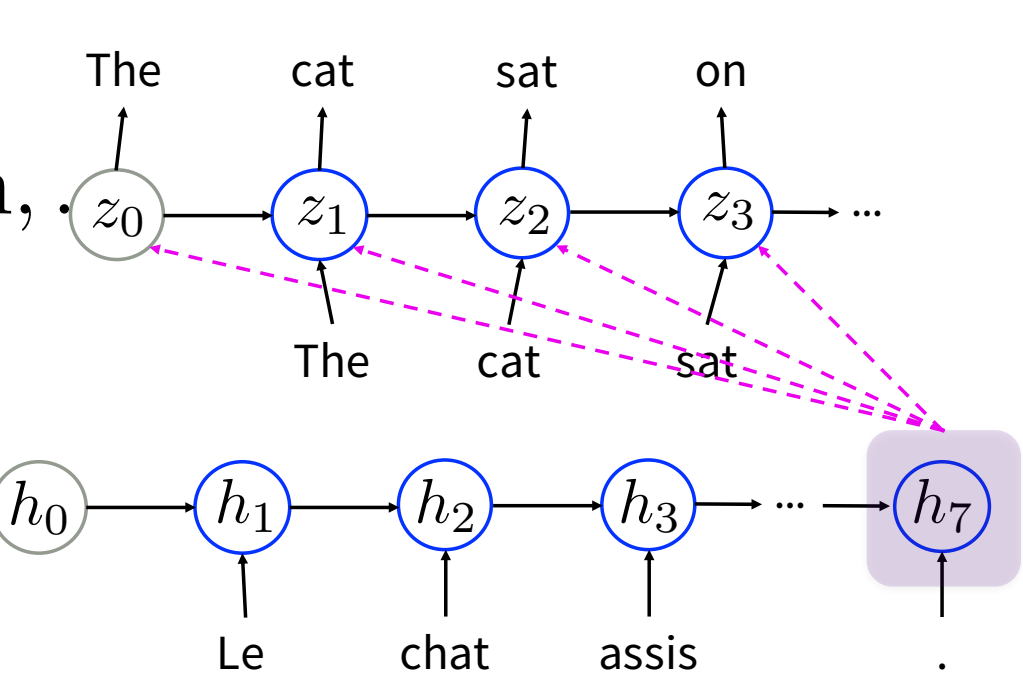




\section{2d. Decoding Strategies}

Ancestral sampling, greedy decoding and beam search 


\section{Decoding (0) - Exhaustive Search}

- Simple and exact decoding algorithm

- Score each and every possible translation

- Pick the best one

DO NOT EVEN THINK of TRYING IT OUT!*

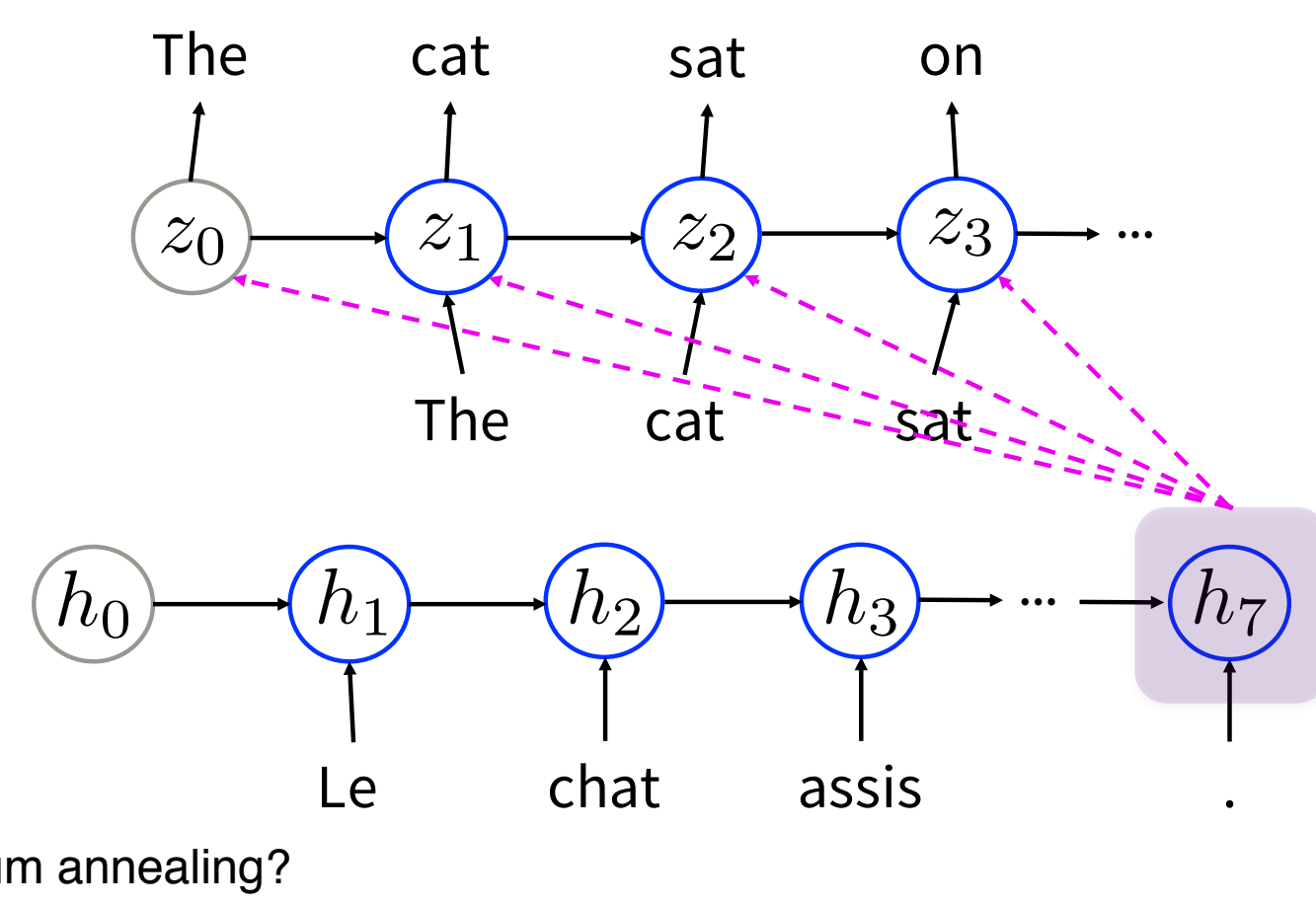

* Perhaps with quantum computer and quantum annealing? 


\section{Decoding (1) - Ancestral Sampling}

- Efficient, unbiased sampling

- One symbol at a time from $\tilde{x}_{t} \sim x_{t} \mid x_{t-1}, \ldots, x_{1}, Y$

- Until $\tilde{x}_{t}=\langle\operatorname{eos}\rangle$ The cat sat

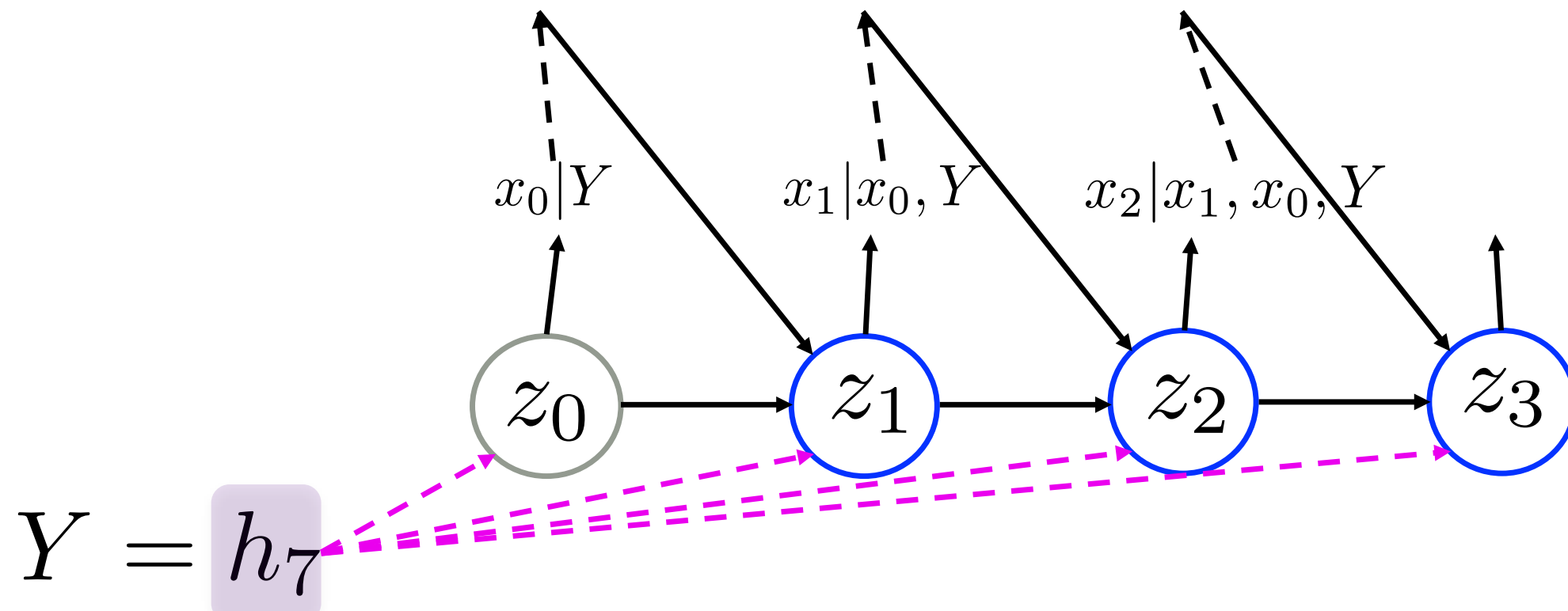




\section{Decoding (1) - Ancestral Sampling}

- Pros:

1. Unbiased (asymptotically exact)

- Cons:

1. High variance

2. Pretty inefficient

\section{The}

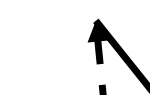

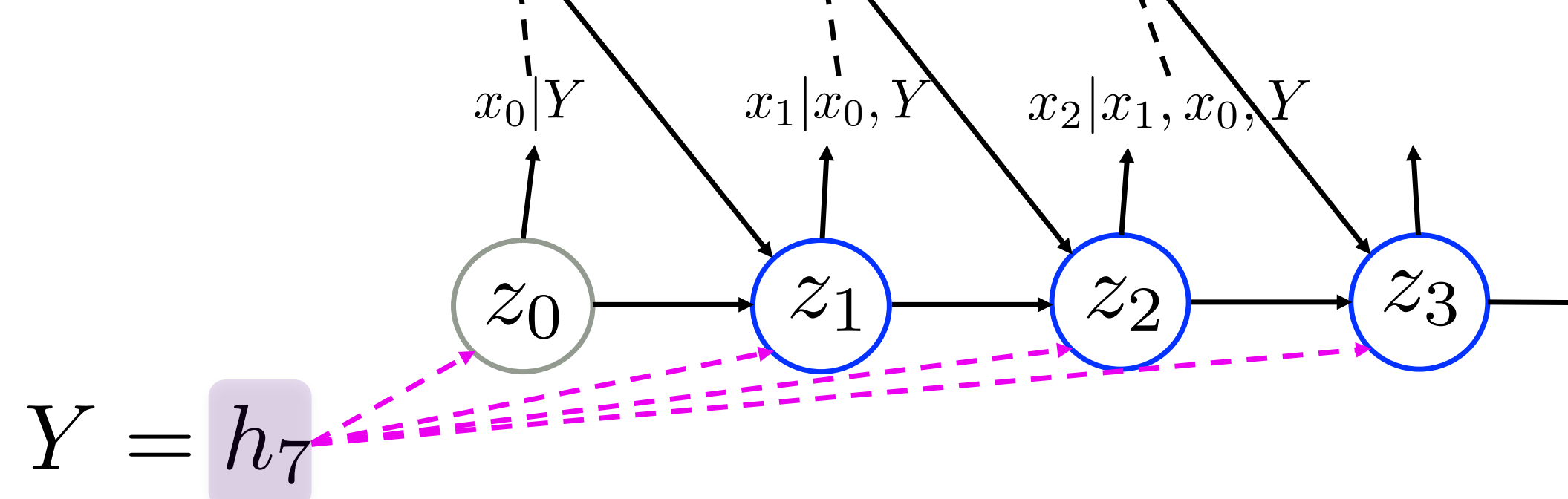




\section{Decoding (2) - Greedy Search}

- Efficient, but heavily suboptimal search

- Pick the most likely symbol each time

$$
\tilde{x}_{t}=\arg \max _{x} \log p\left(x \mid x_{<t}, Y\right)
$$

- Until $\tilde{x}_{t}=\langle$ eos $\rangle$

- Pros:

1. Super-efficient

- Both computation and memory

- Cons:

1. Heavily suboptimal

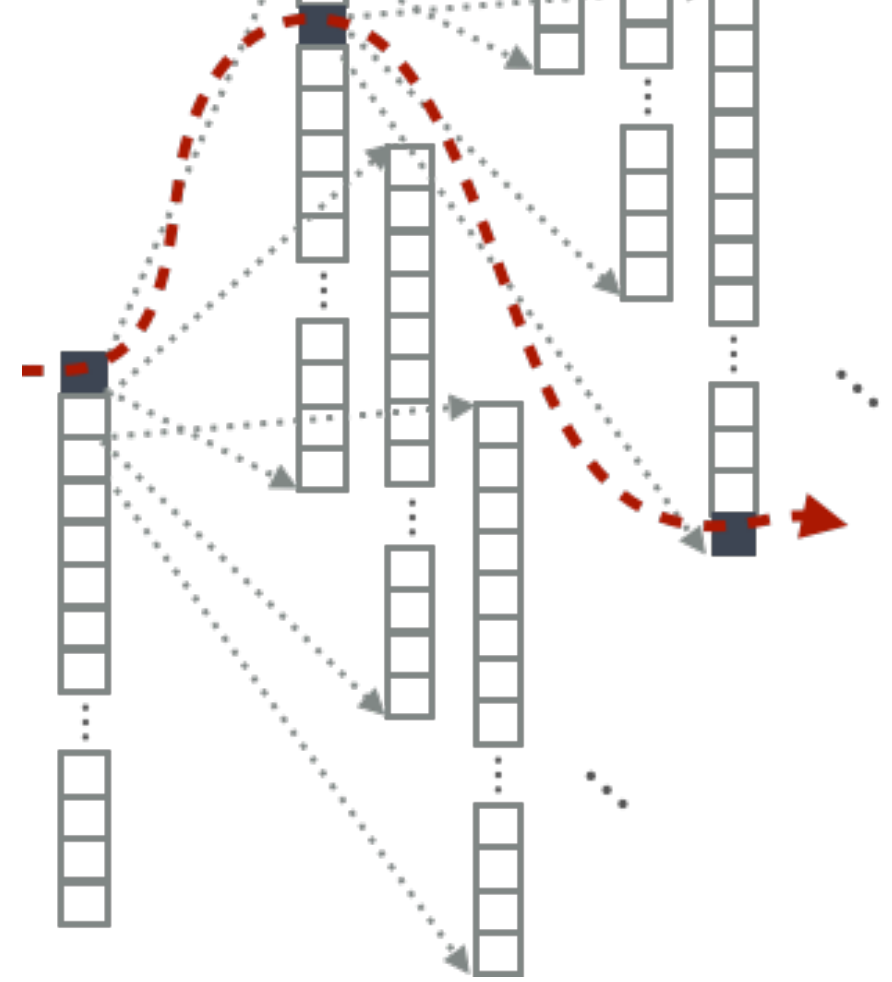




\section{Decoding (3) \\ - Beam Search}

- Pretty, but not quite efficient

- Maintain $K$ hypotheses at a time

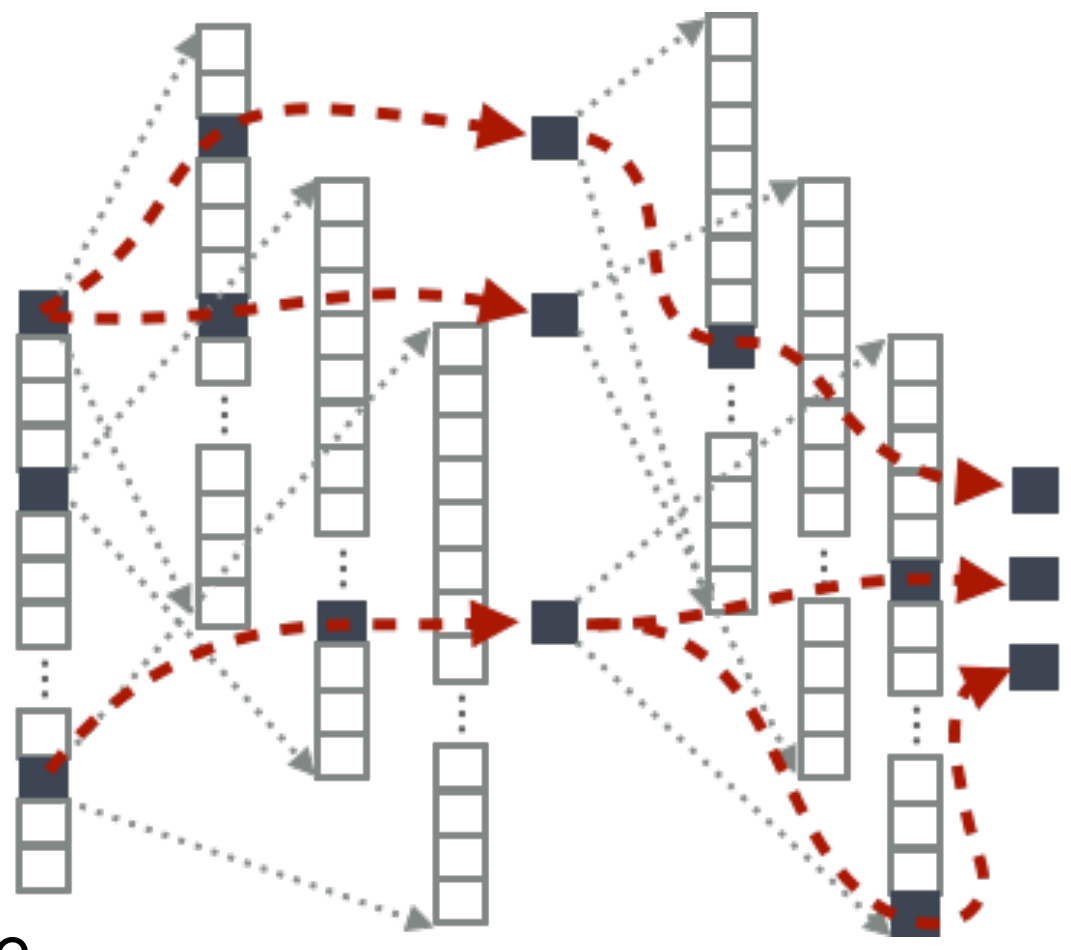

$$
\mathcal{H}_{t-1}=\left\{\left(\tilde{x}_{1}^{1}, \tilde{x}_{2}^{1}, \ldots, \tilde{x}_{t-1}^{1}\right),\left(\tilde{x}_{1}^{2}, \tilde{x}_{2}^{2}, \ldots, \tilde{x}_{t-1}^{2}\right), \ldots,\left(\tilde{x}_{1}^{K}, \tilde{x}_{2}^{K}, \ldots, \tilde{x}_{t-1}^{K}\right)\right\}
$$

- Expand each hypothesis $\mathcal{H}_{t}^{k}=\left\{\left(\tilde{x}_{1}^{k}, \tilde{x}_{2}^{k}, \ldots, \tilde{x}_{t-1}^{k}, v_{1}\right),\left(\tilde{x}_{1}^{k}, \tilde{x}_{2}^{k}, \ldots, \tilde{x}_{t-1}^{k}, v_{2}\right), \ldots,\left(\tilde{x}_{1}^{k}, \tilde{x}_{2}^{k}, \ldots, \tilde{x}_{t-1}^{k}, v_{|V|}\right)\right\}$

- Pick top-K hypotheses from the union $\mathcal{H}_{t}=\cup_{k=1}^{K} \mathcal{B}_{k}$, where $\mathcal{B}_{k}=\underset{\tilde{X} \in \mathcal{A}_{k}}{\arg \max } \log p(\tilde{X} \mid Y), \mathcal{A}_{k}=\mathcal{A}_{k-1}-\mathcal{B}_{k-1}$, and $\mathcal{A}_{1}=\cup_{k^{\prime}=1}^{K} \mathcal{H}_{t}^{k^{\prime}}$. 


\section{Decoding (3) \\ - Beam Search}

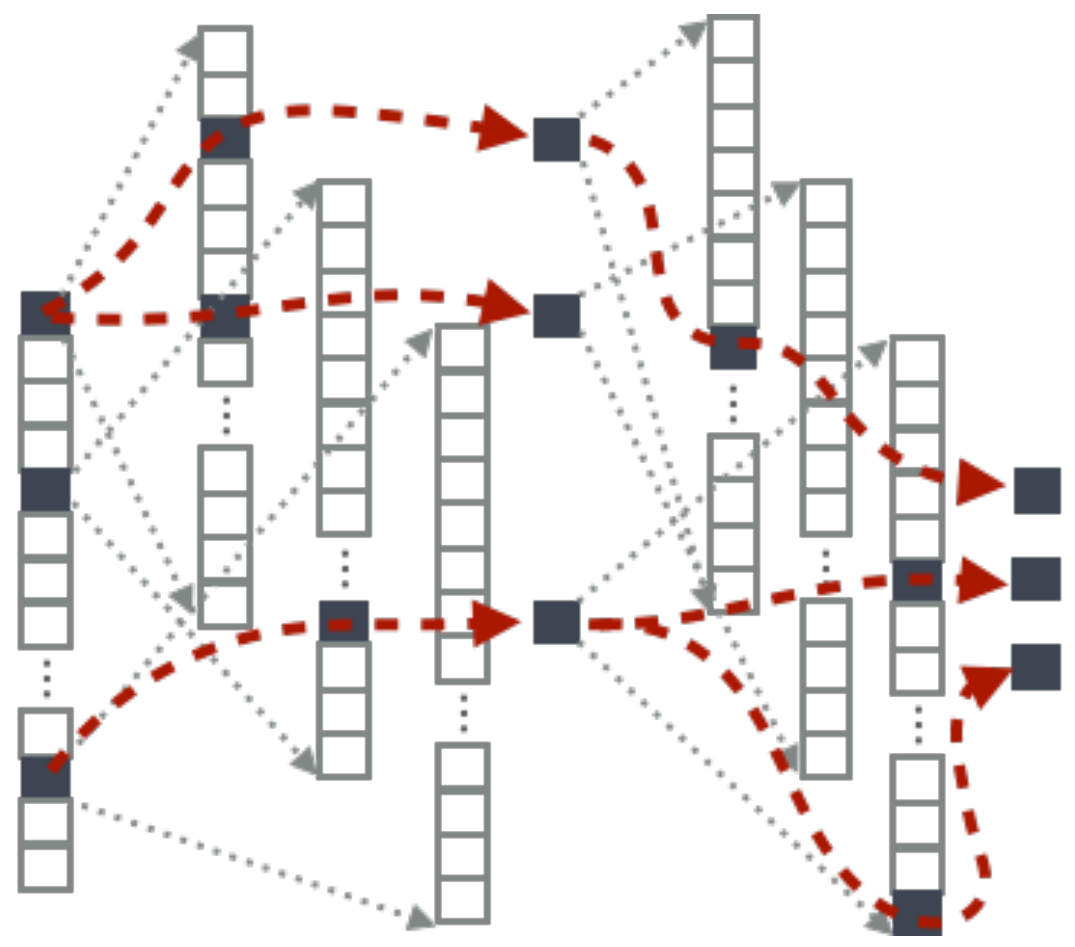

- Asymptotically exact, as $K \rightarrow \infty$

- But, not necessarily monotonic improvement w.r.t. $K$

- $K$ should be selected to maximize the translation quality on a validation set. 


\section{Decoding}

- En-Cz: $12 \mathrm{~m}$ training sentence pairs

\begin{tabular}{cc|cc|cc}
\hline \multirow{2}{*}{ Strategy } & \multirow{2}{*}{ \# Chains } & \multicolumn{2}{c|}{ Valid Set } & \multicolumn{2}{c}{ Test Set } \\
\cline { 3 - 6 } & & NLL & BLEU & NLL & BLEU \\
\hline Ancestral Sampling & 50 & 22.98 & 15.64 & 26.25 & 16.76 \\
Greedy Decoding & - & 27.88 & 15.50 & 26.49 & 16.66 \\
\hline Beamsearch & 5 & 20.18 & 17.03 & 22.81 & 18.56 \\
Beamsearch & 10 & 19.92 & 17.13 & 22.44 & 18.59 \\
\hline
\end{tabular}




\section{Decoding}

- Greedy Search

- Computationally efficient

- Not great quality
- Beam Search

- Computationally expensive

- Not easy to parallelize

- Much better quality

\section{Is there anything in-between?}


2d. Ensemble of Neural MT

Decoding from an ensemble of encoder-decoder's. 


\section{Ensemble of Conditional Recurrent LM}

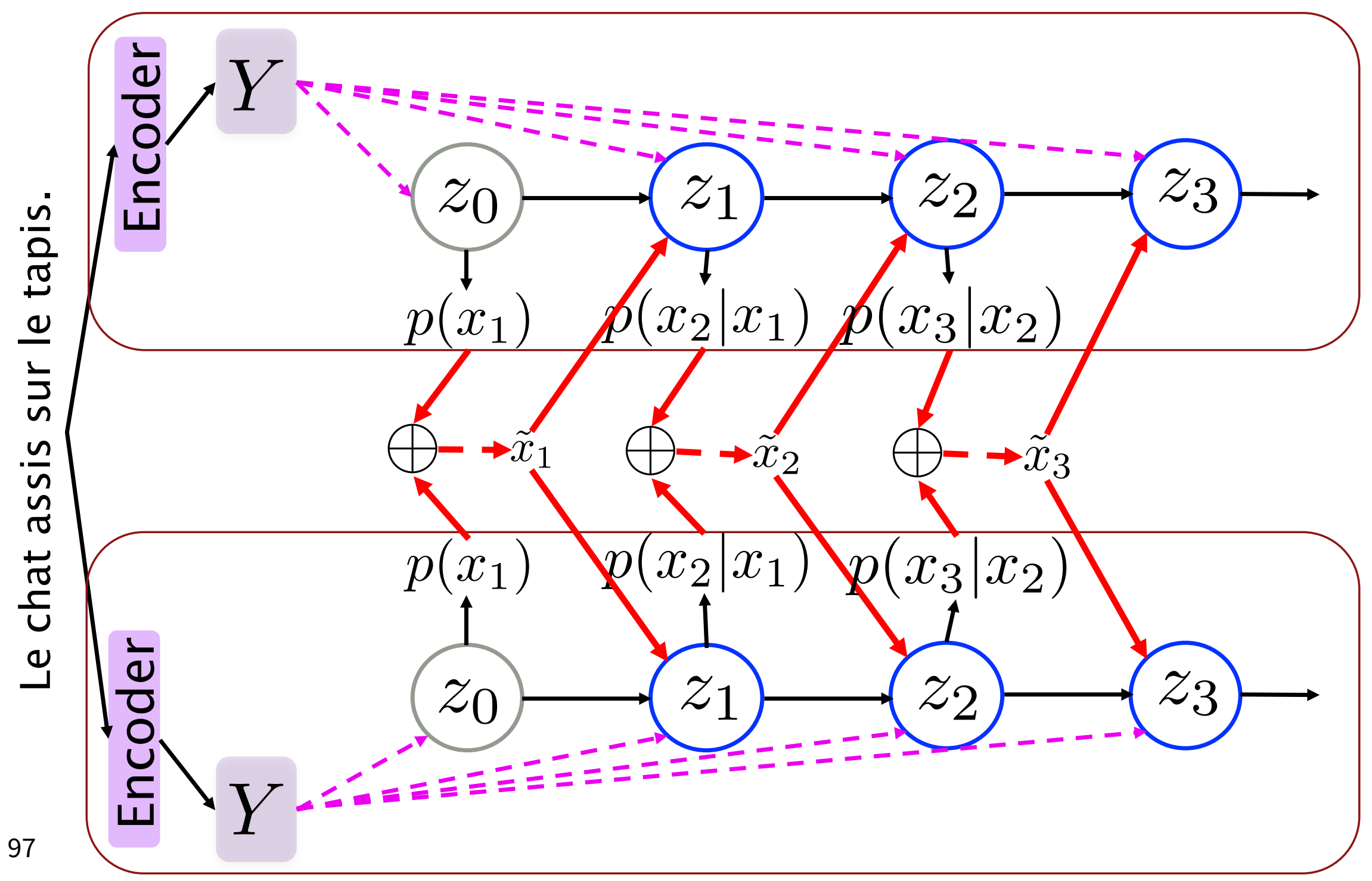




\section{Ensemble of Conditional Recurrent LM}

- Step-wise Ensemble: $p\left(x_{t}^{\mathrm{ens}} \mid x_{<t}^{\mathrm{ens}}, Y\right)=\oplus_{m=1}^{M} p\left(x_{t}^{m} \mid x_{<t}^{m}, Y\right)$

- Ensemble operator $\bigoplus$ implementations

1. Majority voting scheme $(\mathrm{OR})$ :

2. Consensus building scheme (AND):

$$
\oplus_{m=1}^{M} p^{\mathrm{ens}}=\frac{1}{M} \sum_{m=1}^{M} p^{m}
$$

$$
\oplus_{m=1}^{M} p^{\mathrm{ens}}=\left(\prod_{m=1}^{M} p^{m}\right)^{1 / M}
$$

27

25
23
21
19
17
15

- Single (med)

En-De En-Cs En-Ru En-Fi [Jung, Cho \& Bengio, ACL2016] 


\section{Wrap up}

1. Training a recurrent language model efficiently

2. Building a better model with gated recurrent units

3. Building a conditional recurrent language model

4. Generating a translation from a trained conditional recurrent language model 


\section{Do I smell coffee..?}




\section{Have we convinced you about NMT?}

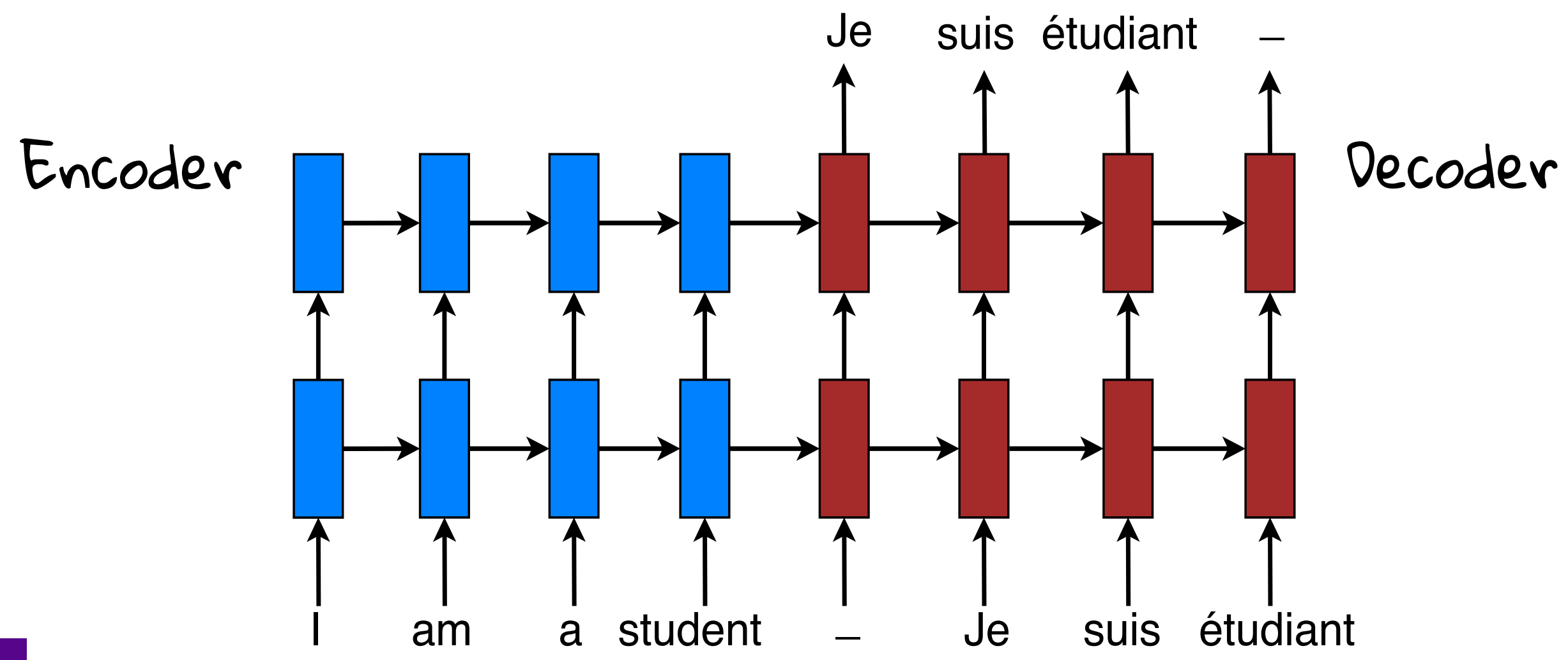




\section{Advancing NMT}

a. The vocabulary aspect

- Goal: extend the vocabulary coverage.

b. The memory aspect

- Goal: translate long sentences better.

c. The language complexity aspect

- Goal: handle more language variations.

d. The data aspect

- Goal: utilize more data sources. 
3. Advancing NMT

a. The vocabulary aspect

- Goal: extend the vocabulary coverage.

b. The memory aspect

- Goal: translate long sentences better.

c. The language complexity aspect

- Goal: handle more language variations.

d. The data aspect

- Goal: utilize more data sources. 


\section{The word generation problem}

Softmax Hidden parameters state
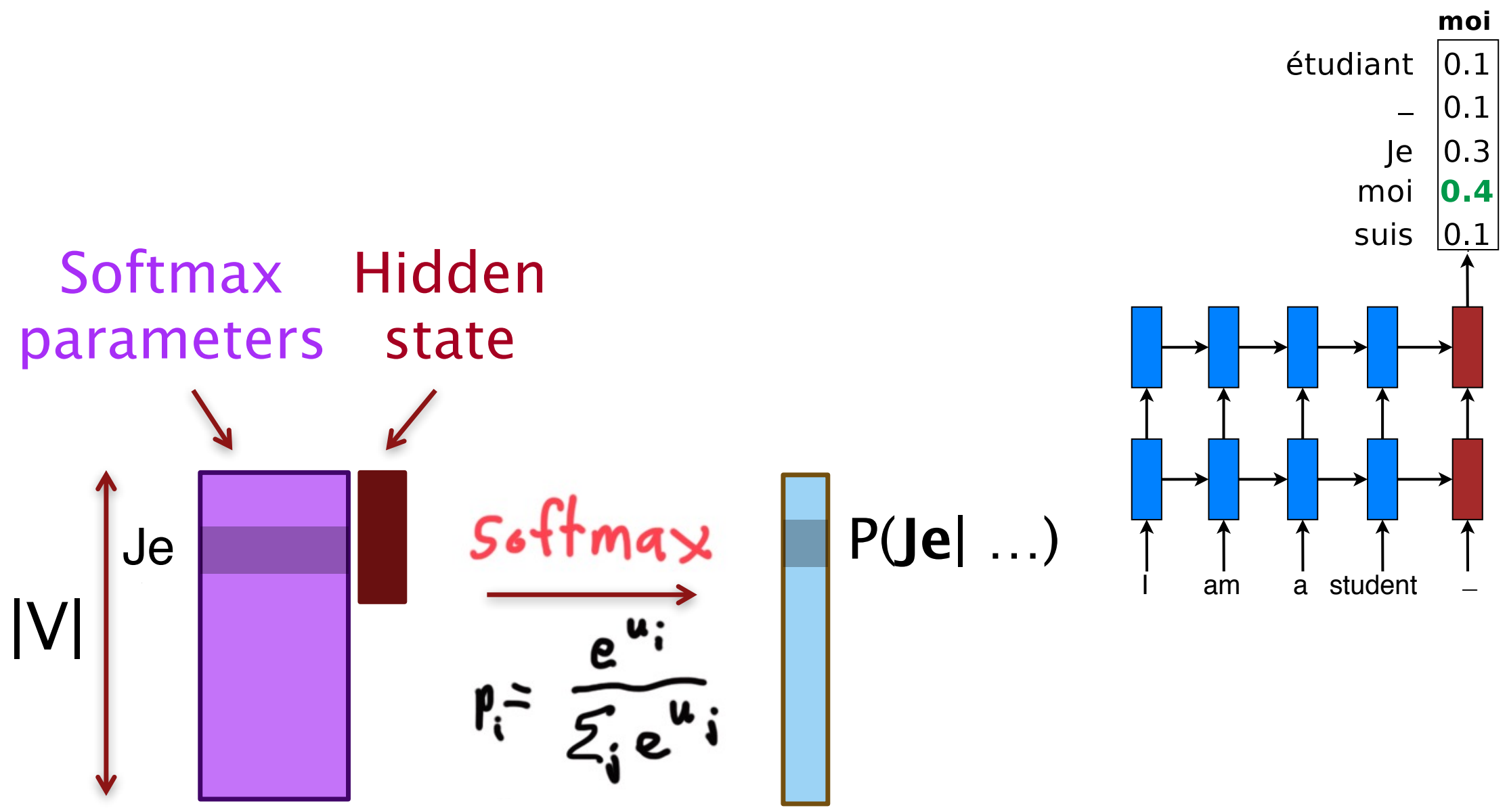
0.1$$
-0.1
$$$$
0.3
$$$$
0.4
$$$$
\text { suis } 0.1
$$

parameters state




\section{The word generation problem}

- Word generation problem

$\begin{array}{cc} & \mathbf{m o i} \\ \text { étudiant } & 0.1\end{array}$

$-0.1$

Je 0.3

moi 0.4

suis 0.1

Softmax Hidden

parameters state

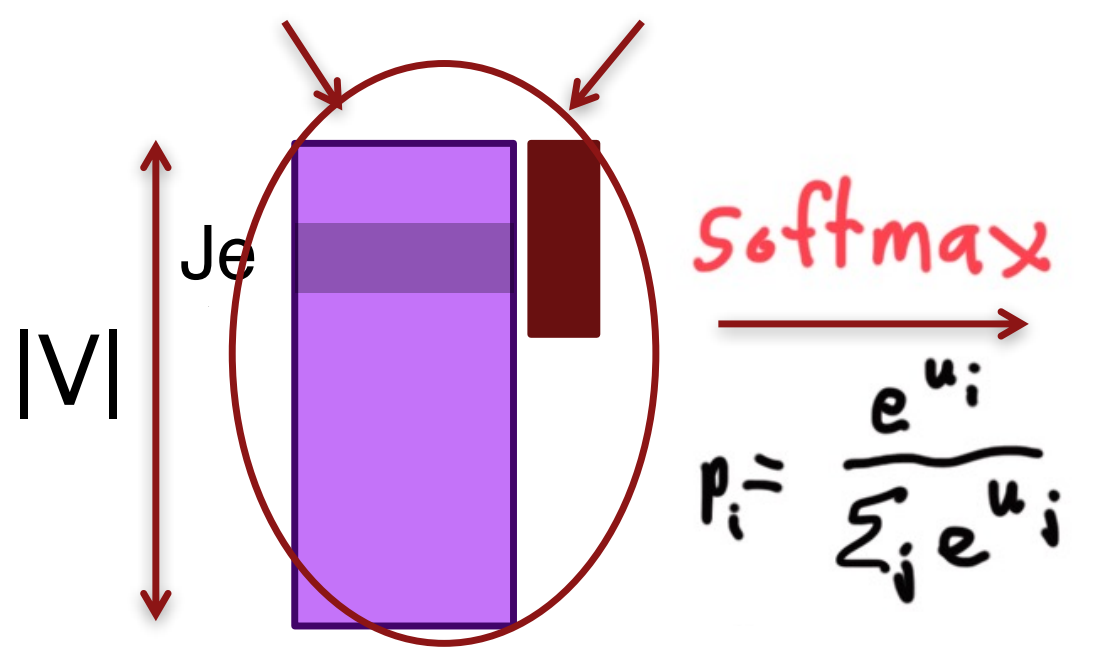

$\mathrm{P}(\mathrm{Je} \mid \ldots)$

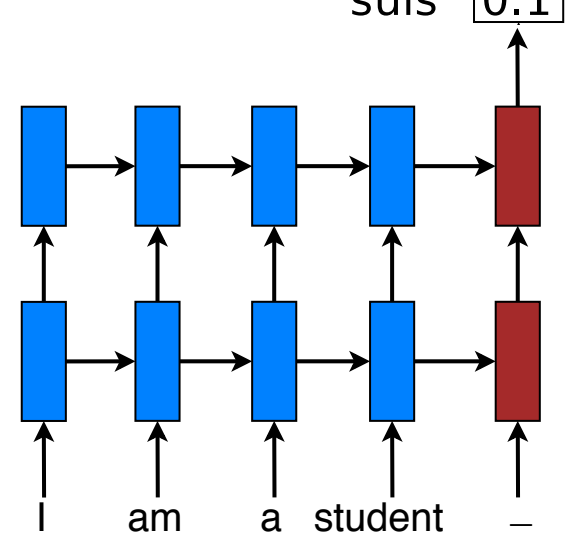

Softmax computation is expensive. 


\section{The word generation problem}

- Word generation problem

- Vocabs are modest: 50K.

The ecotax portico in Pont-de-Buis Le portique écotaxe de Pont-de-Buis

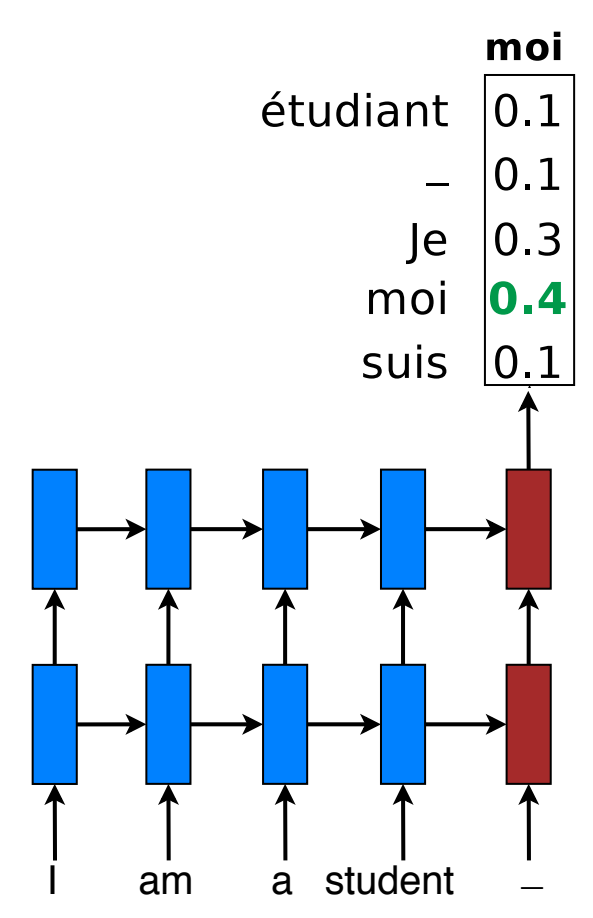

The <unk> portico in <unk> Le <unk> <unk> de <unk> 


\section{First thought: scale the softmax}

- Lots of ideas from the neural LM literature!

- Hierarchical models: tree-structured vocabulary

- [Morin \& Bengio, AISTATS'05], [Mnih \& Hinton, NIPS'09].

- Complex, sensitive to tree structures.

- Noise-contrastive estimation: binary classification

- [Mnih \& Teh, ICML'12], [Vaswani et al., EMNLP'13].

- Different noise samples per training example.*$$
\text { Not GPU-friendly }
$$ 


\section{Large-vocab NMT}

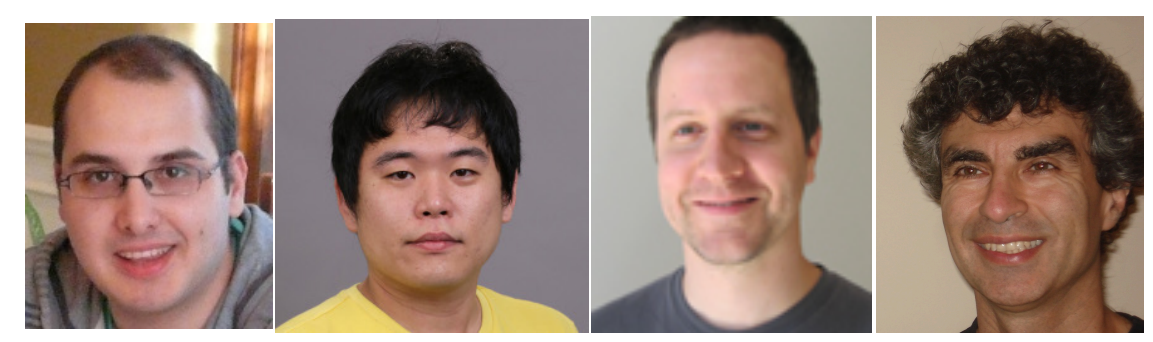

- GPU-friendly.

- Training: a subset of the vocabulary at a time.

- Testing: smart on the set of possible translations.

\section{Fast at both train \& test time.}

Sébastien Jean, Kyunghyun Cho, Roland Memisevic, Yoshua Bengio. On Using Very Large Target Vocabulary for Neural Machine Translation. ACL'15. 


\section{Training}

- Each time train on a smaller vocab $\mathrm{V}^{\prime} \ll \mathrm{V}$

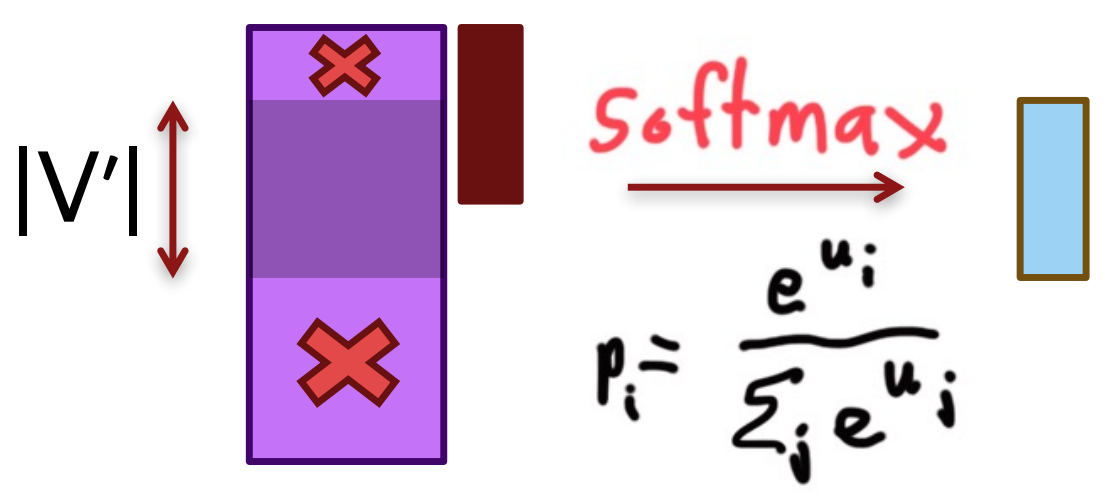

How do we select V? 


\section{Training}

- Each time train on a smaller vocab $\mathrm{V}^{\prime} \ll \mathrm{V}$

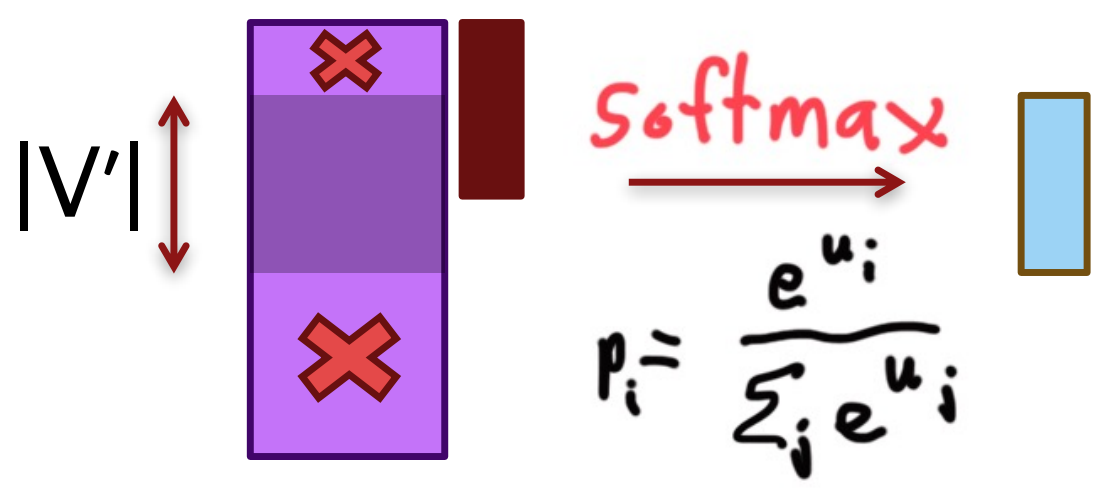

- Partition training data in subsets:

- Each subset has $\tau$ distinct target words, $\left|V^{\prime}\right|=\tau$. 


\section{Training - Segment data}

- Sequentially select examples: $\left|V^{\prime}\right|=5$.

she loves cats he likes dogs

cats have tails dogs have tails dogs chase cats she loves dogs cats hate dogs

$$
V^{\prime}=\{\text { she, loves, cats, he, likes }\}
$$




\section{Training - Segment data}

- Sequentially select examples: $\left|V^{\prime}\right|=5$.

she loves cats

he likes dogs

cats have tails dogs have tails

$V^{\prime}=\{$ cats, have, tails, dogs, chase $\}$ dogs chase cats

she loves dogs

cats hate dogs 


\section{Training - Segment data}

- Sequentially select examples: $\left|V^{\prime}\right|=5$.

she loves cats he likes dogs cats have tails dogs have tails dogs chase cats she loves dogs cats hate dogs

$$
V^{\prime}=\{\text { she, loves, dogs, cats, hate }\}
$$

- Practice: $|\mathrm{V}|=500 \mathrm{~K},\left|\mathrm{~V}^{\prime}\right|=30 \mathrm{~K}$ or $50 \mathrm{~K}$. 


\section{Testing - Select candidate words}

- K most frequent words: unigram prob.

de,

la

et

des

les 


\section{Testing - Select candidate words}

- K most frequent words: unigram prob.

de,

- Candidate target words

- K' choices per source word. $\mathrm{K}^{\prime}=3$.

la

et des les
elle
aime
chats
celle amour
chat
ceci
aimer
félin
She
loves
cats 


\section{Testing - Select candidate words}

$\mathbf{K}^{\prime}$

$\begin{array}{llll}\text { elle } & \text { aime } & \text { chats } \\ \text { celle } & \text { amour } & \text { chat } \\ \text { ceci } & \text { aimer } & \text { félin }\end{array} \quad+\begin{aligned} & \text { la } \\ & \text { et } \\ & \text { She }\end{aligned}$

- Produce translations within the candidate list

- Practice: $\mathrm{K}^{\prime}=10$ or $20, \mathrm{~K}=15 \mathrm{k}, 30 \mathrm{k}$, or $50 \mathrm{k}$. 


\section{More on large-vocab techniques}

- "BlackOut: Speeding up Recurrent Neural Network Language Models with very Large Vocabularies" - [Ji, Vishwanathan, Satish, Anderson, Dubey, ICLR'16].

- Good survey over many techniques.

- "Simple, Fast Noise Contrastive Estimation for Large RNN Vocabularies" - [Zoph, Vaswani, May, Knight, NAACL'16].

- Use the same samples per minibatch. GPU efficient. 


\section{$2^{\text {nd }}$ thought on word generation}

- Scaling softmax is insufficient:

- New names, new numbers, etc., at test time.

- But previous MT models can copy words.

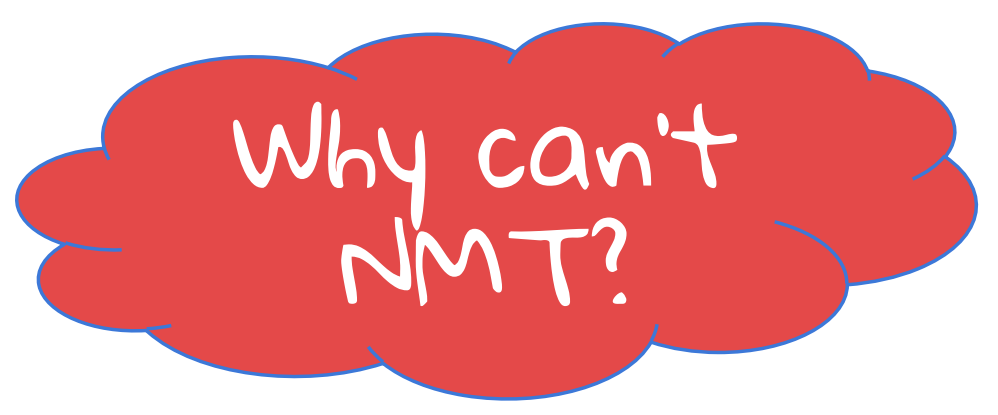




\section{Copy Mechanism}

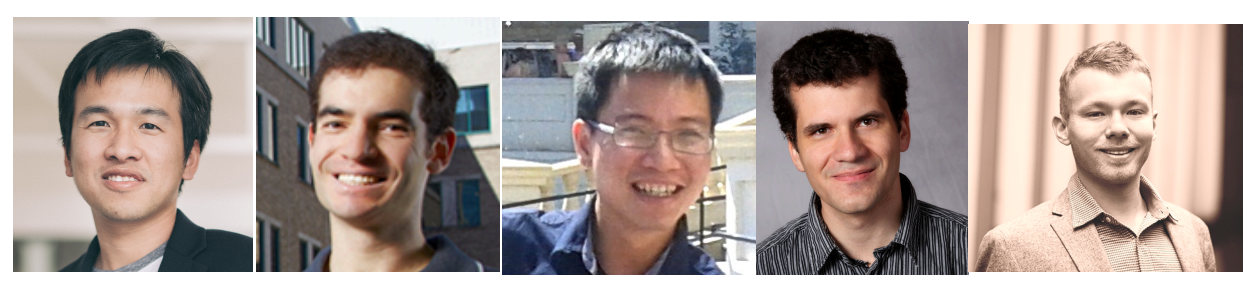

- Simple way to track target $<$ unk>.

- Treat any NMT as a black box.

- Annotate training data.

- Post-process translations.

\section{Complementary to softmax scaling!}

Thang Luong, Ilya Sutskever, Quoc Le, Oriol Vinyals, Wojciech Zaremba. Addressing the Rare Word Problem in Neural Machine Translation. ACL'15. 


\section{Training annotation}

- Learn alignments

The ecotax portico in Pont-de-Buis

Le portique écotaxe de Pont-de-Buis

- Add relative positions

The <unk> portico in <unk>

Le unk unk de unlo 


\section{Training annotation}

- Learn alignments

The ecotax portico in Pont-de-Buis

Le portique écotaxe de Pont-de-Buis

- Add relative positions

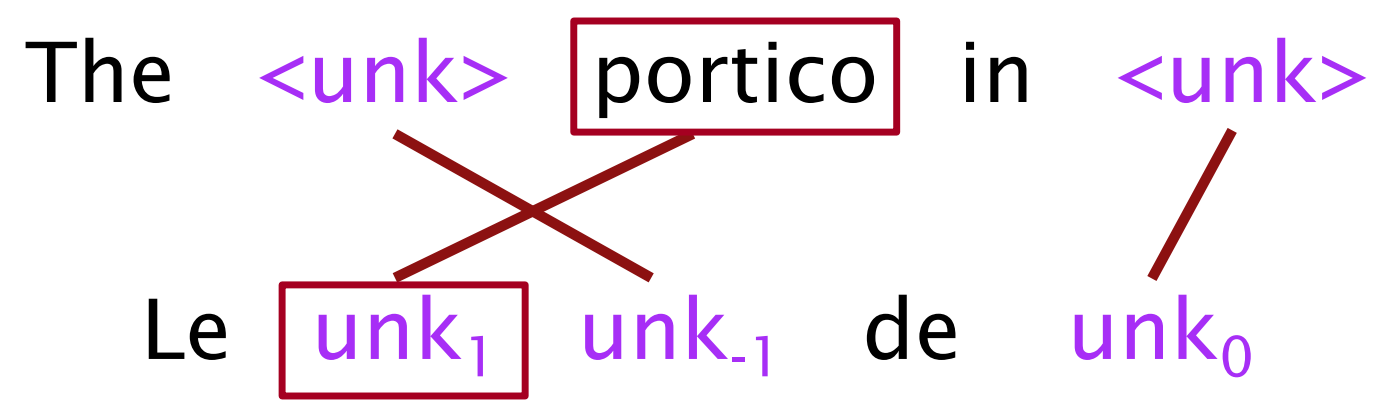




\section{Training annotation}

- Learn alignments

The ecotax portico in Pont-de-Buis

Le portique écotaxe de Pont-de-Buis

- Add relative positions

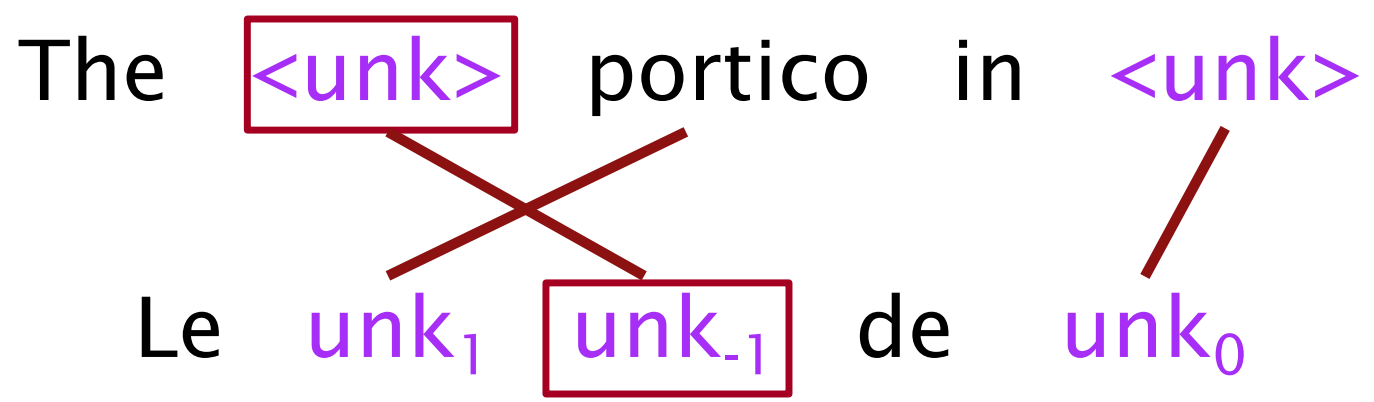




\section{Post-processing}
ecotax
Pont-de-Buis
Test sentence The <unk portico in $<$ unk $>$
Translation
Le portique unk ${ }_{-1}$ de unk $_{0}$ 


\section{Post-processing}

Test sentence The $\begin{aligned} & \text { ecotax } \\ & <\text { unk }>\text { pont-de-Buis }\end{aligned}$

Translation

Le portique $u n k_{-1}$ de $u n k_{0}$

8. Dictionary translation

Post-edit Translation Le portique écotaxe de Pont-de-Buis 


\section{Post-processing}

Test sentence The $\begin{gathered}\text { ecotax } \\
<u n k>\end{gathered}$ portico in \begin{tabular}{c} 
Pont-de-Buis \\
\hline ink $>$
\end{tabular}

Translation

Le portique unk de $_{\text {unk }}$

$\sqrt{ }$ Identity

copy

Post-edit

Translation

Le portique écotaxe de Pont-de-Buis 


\section{Effects of Translating Rare Words}

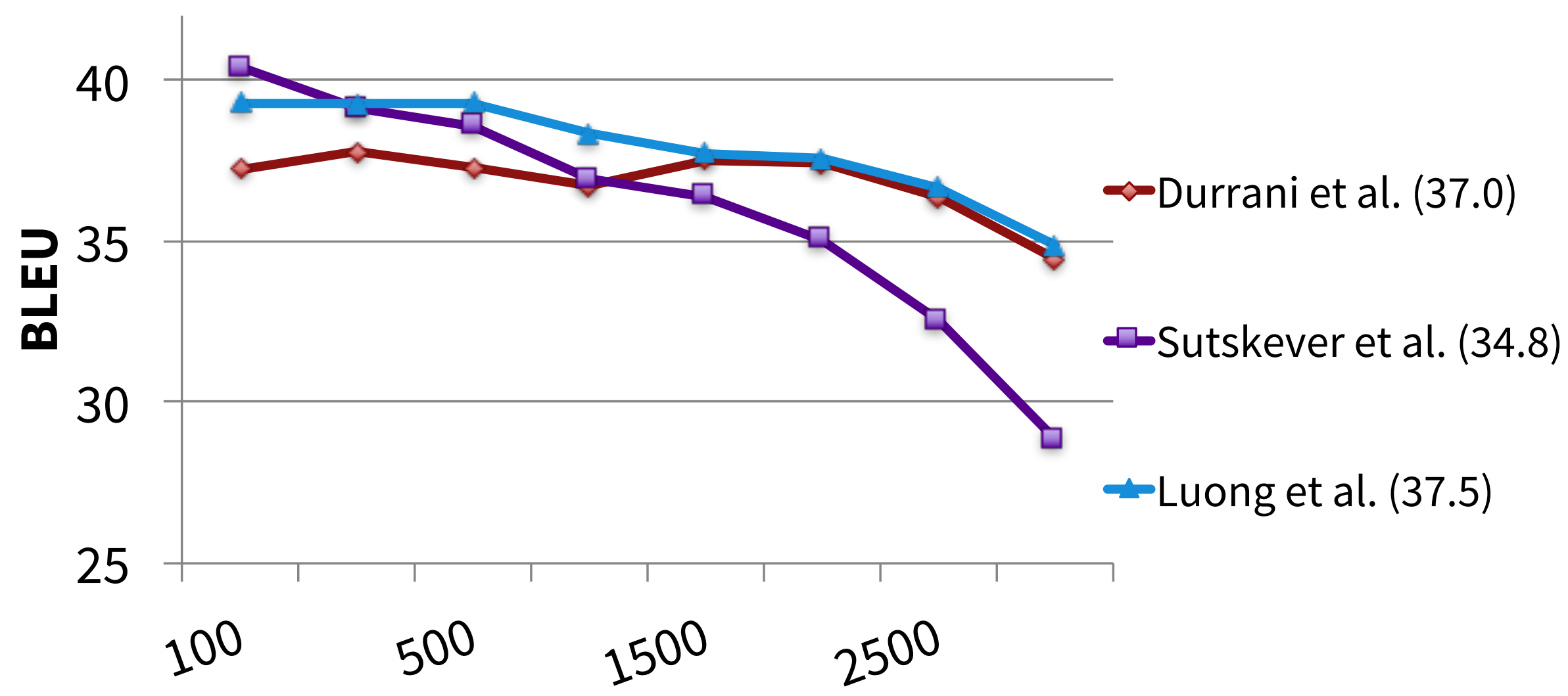

Sentences ordered by average word frequency rank 


\section{Sample translations}

\begin{tabular}{l|l} 
source & $\begin{array}{l}\text { This trader, Richard Usher, left RBS in } 2010 \text { and is understand to } \\
\text { have be given leave from his current position as European head of } \\
\text { forex spot trading at JPMorgan. }\end{array}$ \\
\hline human & $\begin{array}{l}\text { Ce trader, Richard Usher, a quitté RBS en } 2010 \text { et aurait été mis } \\
\text { suspendu de son poste de responsable européen du trading au } \\
\text { comptant pour les devises chez JPMorgan. }\end{array}$ \\
\hline trans & $\begin{array}{l}\text { Ce unk } k_{0}, \text { Richard unk } \\
\text { autorisé à quitter son poste actuel en tant que leader européen du } \\
\text { marché des points de vente au unk }\end{array}$. \\
\hline trans+ \\
unk & $\begin{array}{l}\text { Ce négociateur, Richard Usher, a quitté RBS en } 2010 \text { et a compris } \\
\text { qu' il est autorisé à quitter son poste actuel en tant que leader } \\
\text { européen du marché des points de vente au JPMorgan }\end{array}$
\end{tabular}

- Translates well long sentences 


\section{Copy Mechanism - Old but useful!}

- Later, we'll discuss better techniques!

- But it's useful when adapting to new tasks!

- Text summarization: [Gu, Lu, Li, Li, ACL'16], [Gulcehre, Ahn, Nallapati, Zhou, Bengio, ACL'16]

- Semantic parsing: [Jia, Liang, ACL'16]

\section{Learn to decide when to copy.}


3. Advancing NMT

a. The vocabulary aspect

- Goal: extend the vocabulary coverage.

b. The memory aspect

- Goal: translate long sentences better.

c. The language complexity aspect

- Goal: handle more language variations.

d. The data aspect

- Goal: utilize more data sources. 


\section{Vanilla seq2seq \& long sentences}

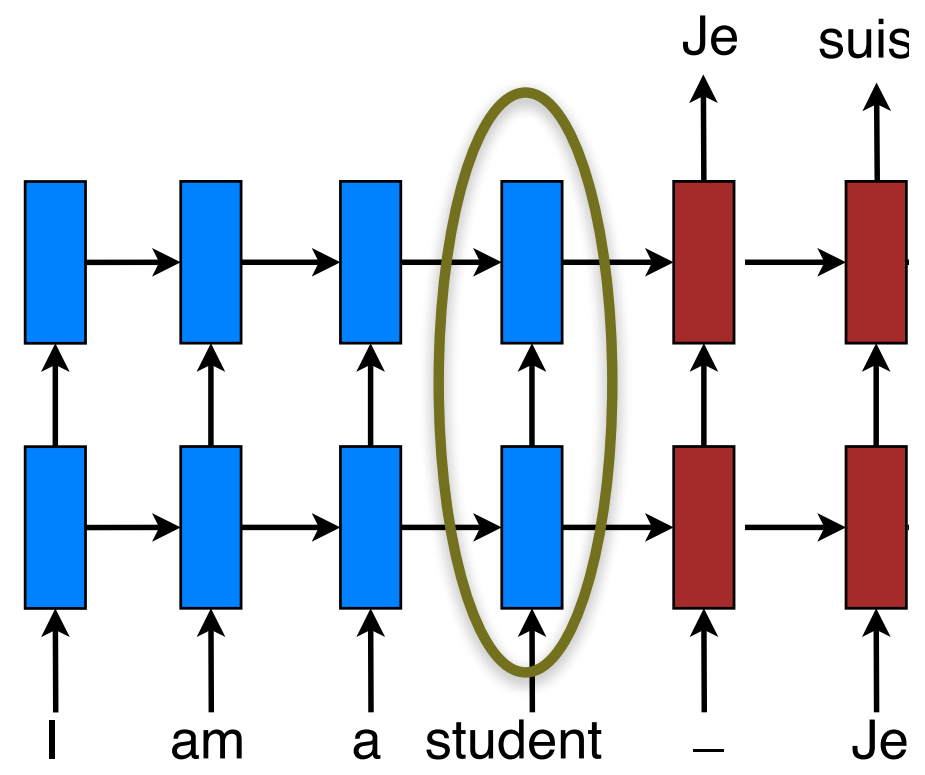

Problem: fixed-dimensional representations 


\section{Attention Mechanism}

Started in computer vision! [Larochelle \& Hinton, 2010], [Denil, Bazzani, Larochelle, Freitas, 2012]

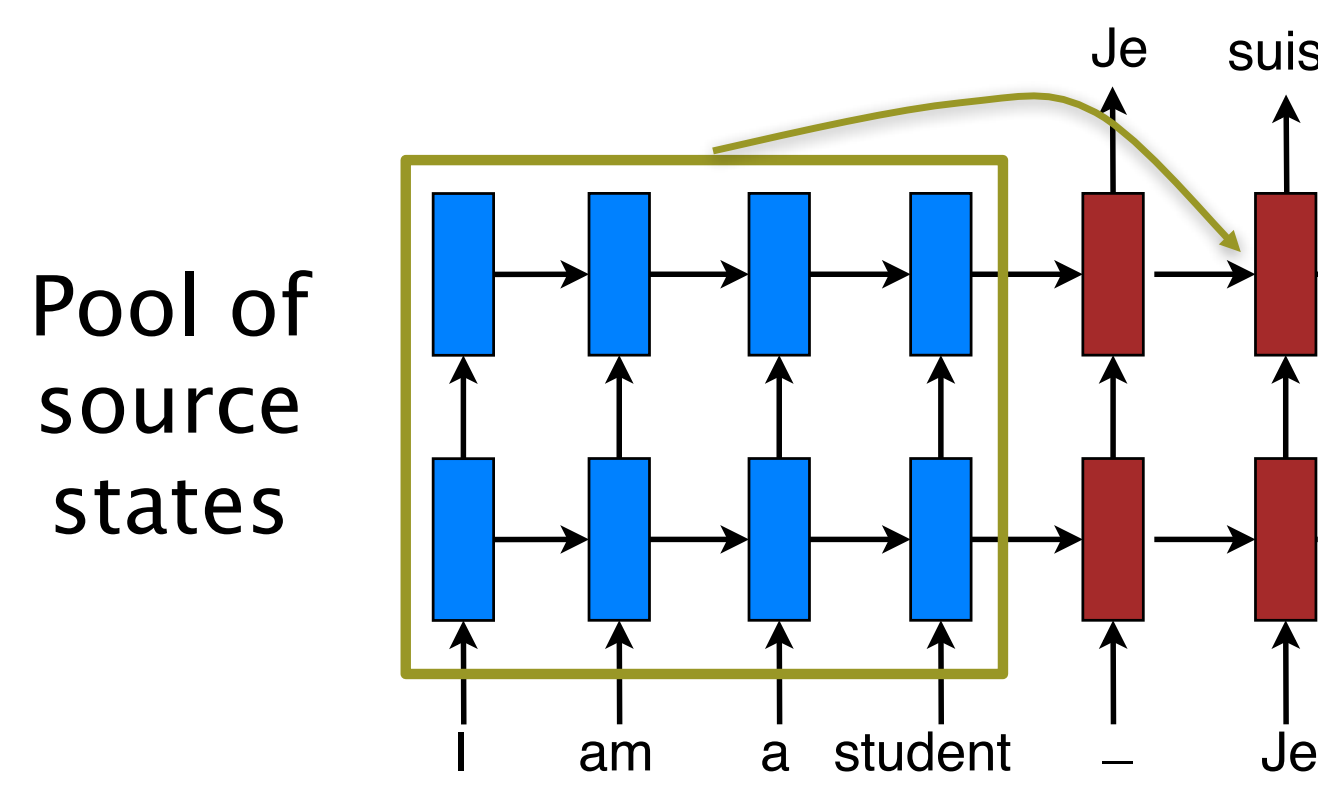

- Solution: random access memory

- Retrieve as needed. 


\section{Learning both translation \& alignment}
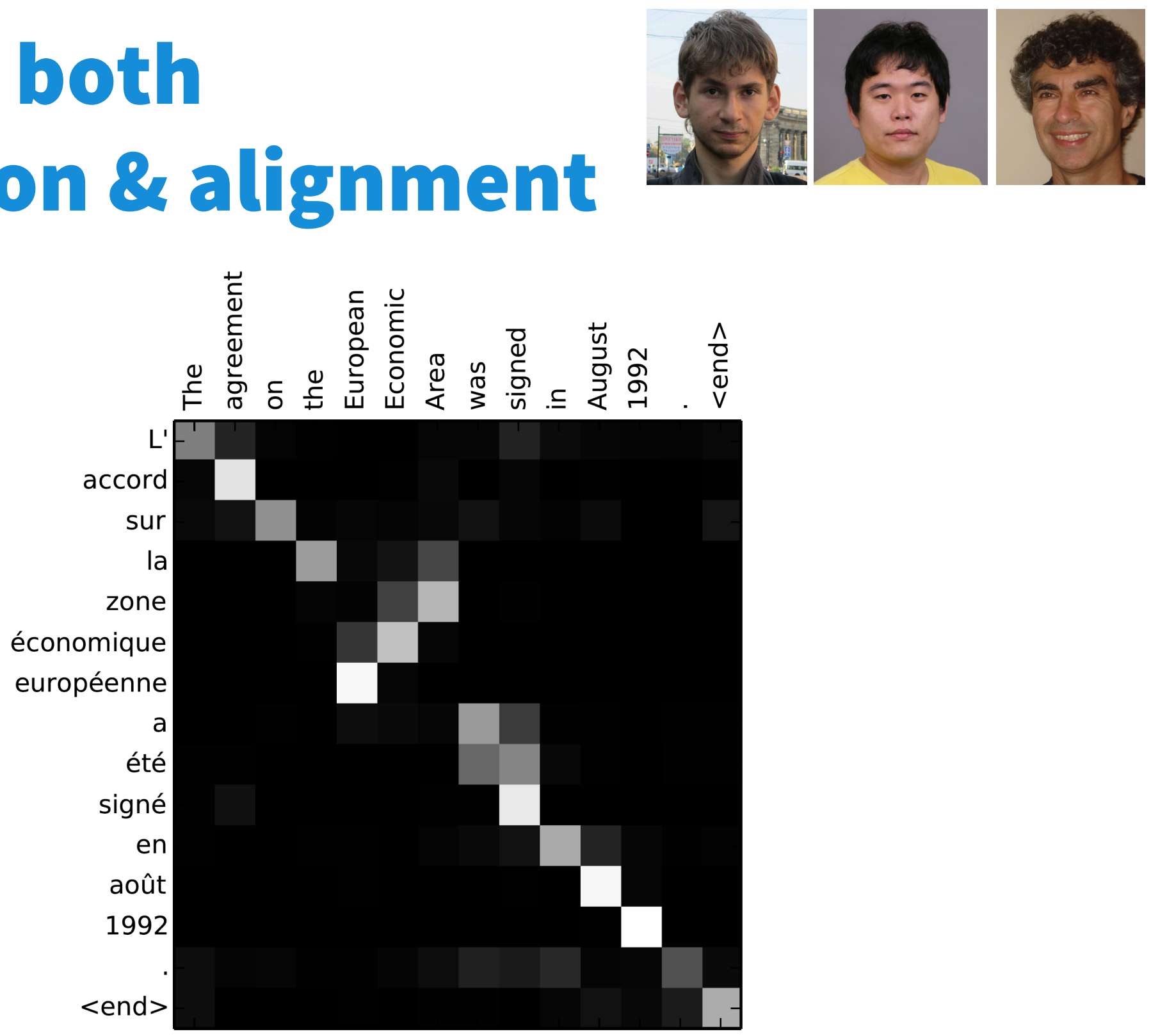

Dzmitry Bahdanau, KyungHuyn Cho, and Yoshua Bengio. Neural Machine Translation by Jointly Learning to Translate and Align. ICLR' 15. 


\section{Attention Mechanism}

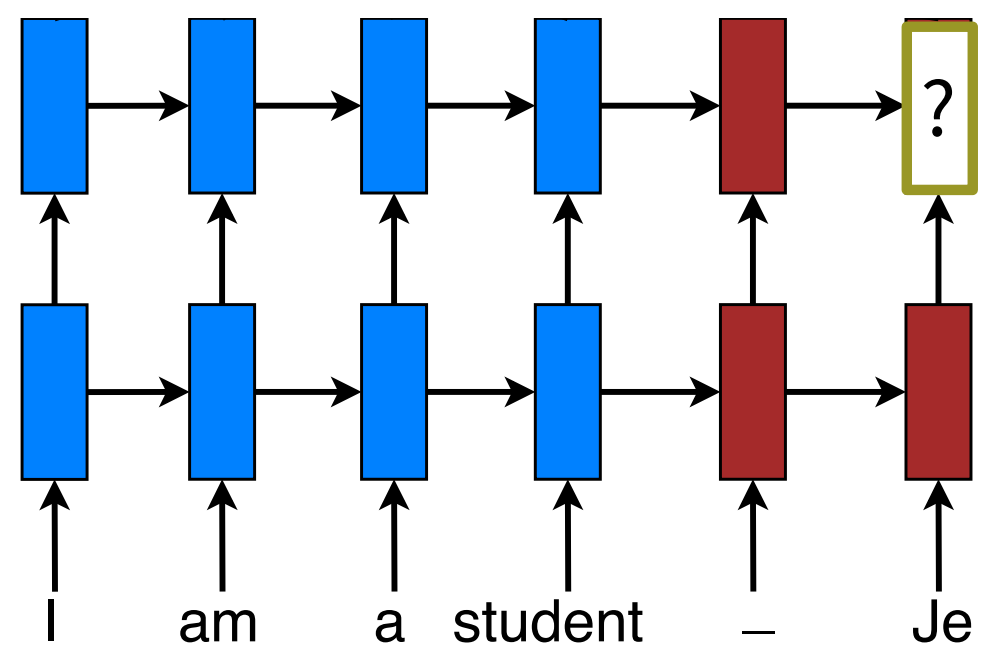

Simplified version of (Bahdanau et al., 2015) 


\section{Attention Mechanism - Scoring}

$$
\operatorname{score}\left(\boldsymbol{h}_{t-1}, \overline{\boldsymbol{h}}_{s}\right)
$$

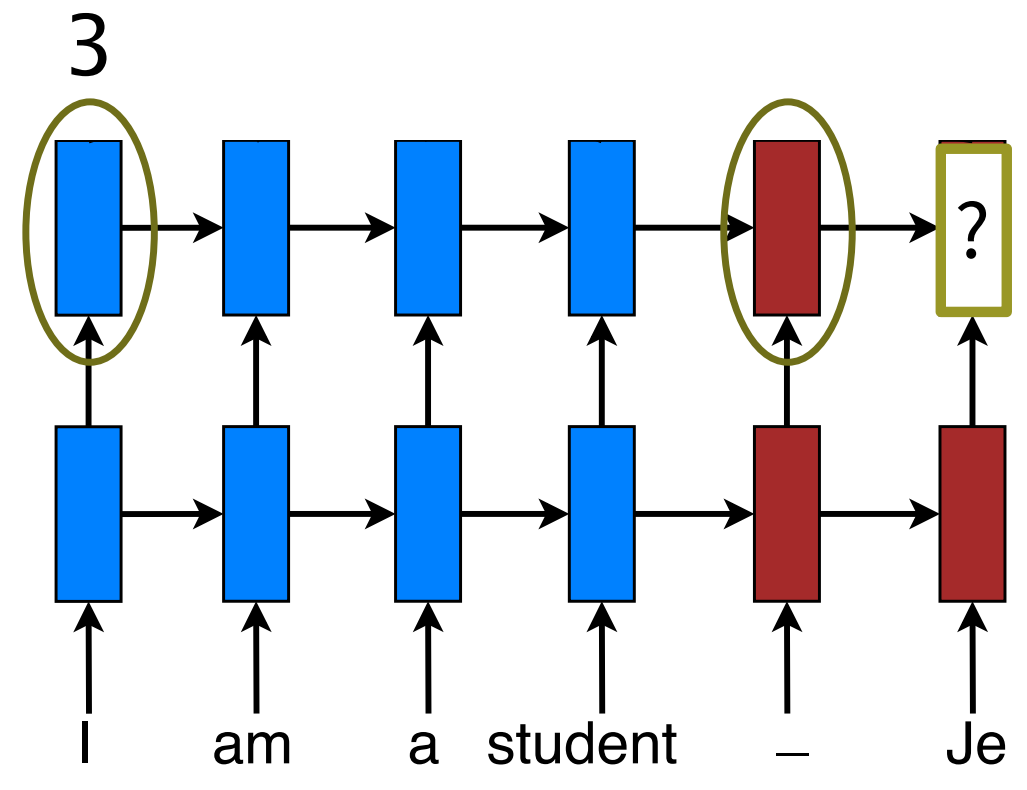

- Compare target and source hidden states. 


\section{Attention Mechanism - Scoring}

$$
\operatorname{score}\left(\boldsymbol{h}_{t-1}, \overline{\boldsymbol{h}}_{s}\right)
$$

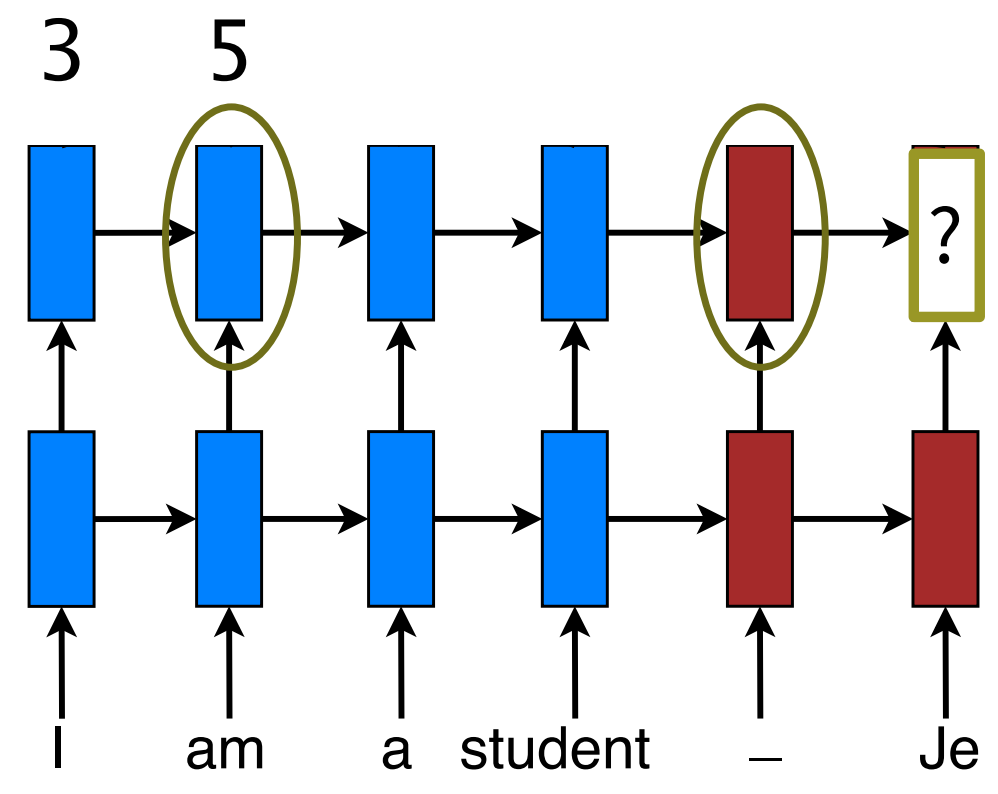

- Compare target and source hidden states. 


\section{Attention Mechanism - Scoring}

$$
\operatorname{score}\left(\boldsymbol{h}_{t-1}, \overline{\boldsymbol{h}}_{s}\right)
$$

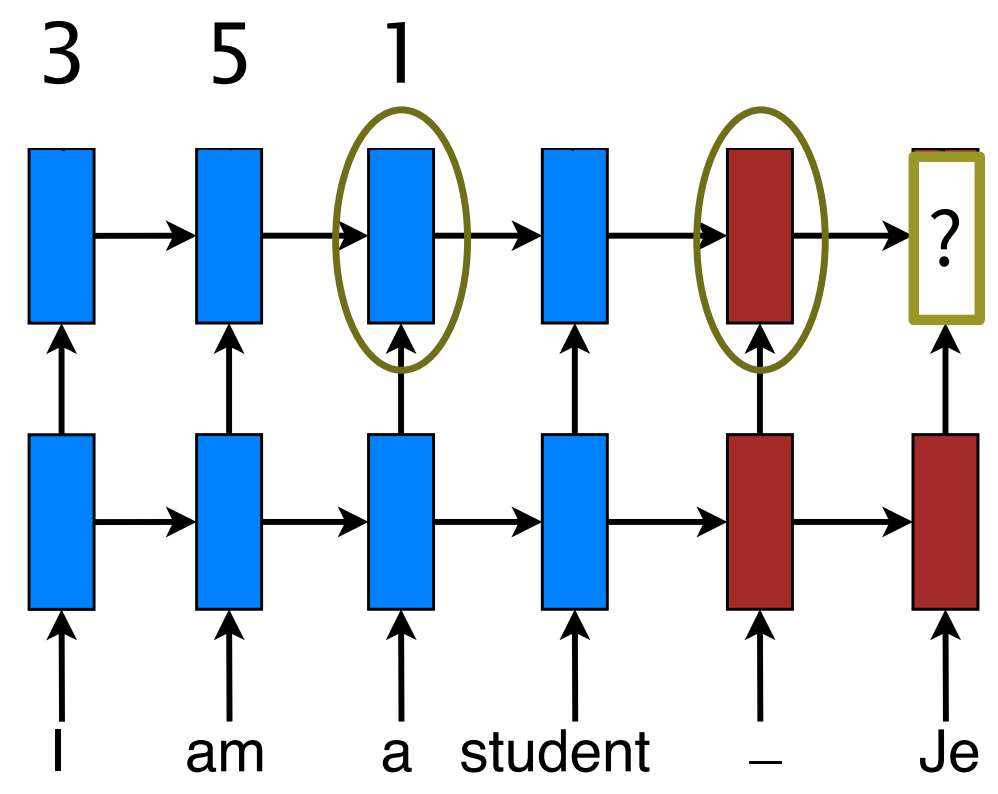

- Compare target and source hidden states. 


\section{Attention Mechanism - Scoring}

$$
\operatorname{score}\left(\boldsymbol{h}_{t-1}, \overline{\boldsymbol{h}}_{s}\right)
$$

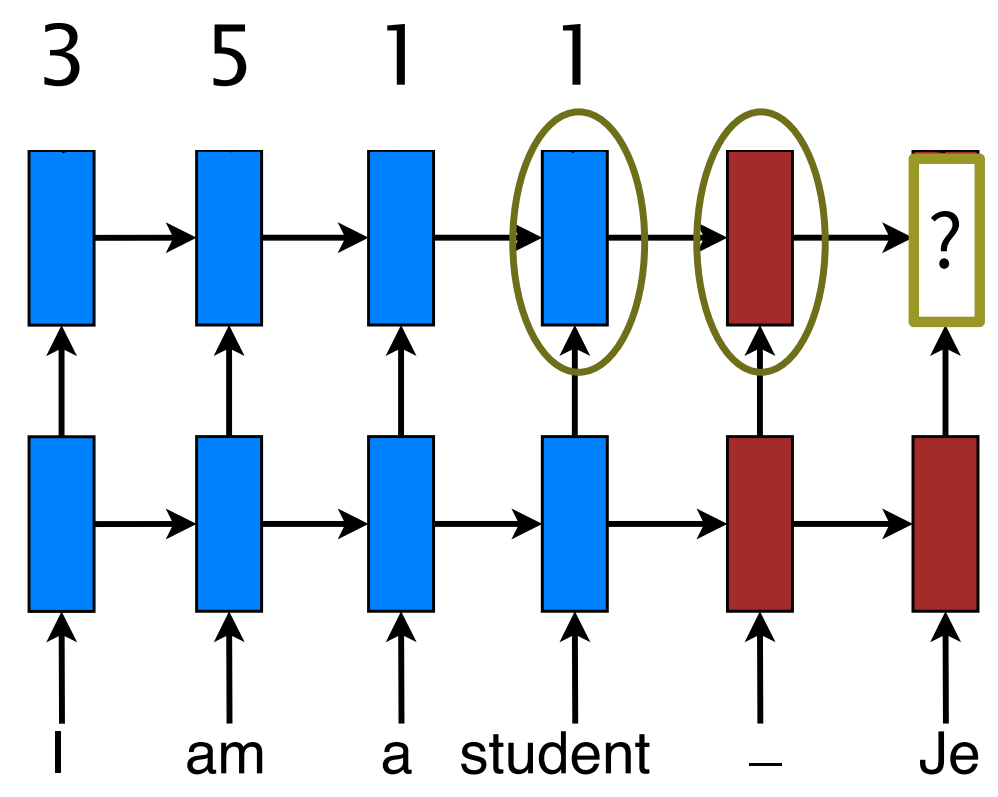

- Compare target and source hidden states. 


\section{Attention Mechanism - Normalization}

$$
\boldsymbol{a}_{t}(s)=\frac{\mathrm{e}^{\mathrm{score}(s)}}{\sum_{s^{\prime}} \mathrm{e}^{\mathrm{score}\left(s^{\prime}\right)}}
$$

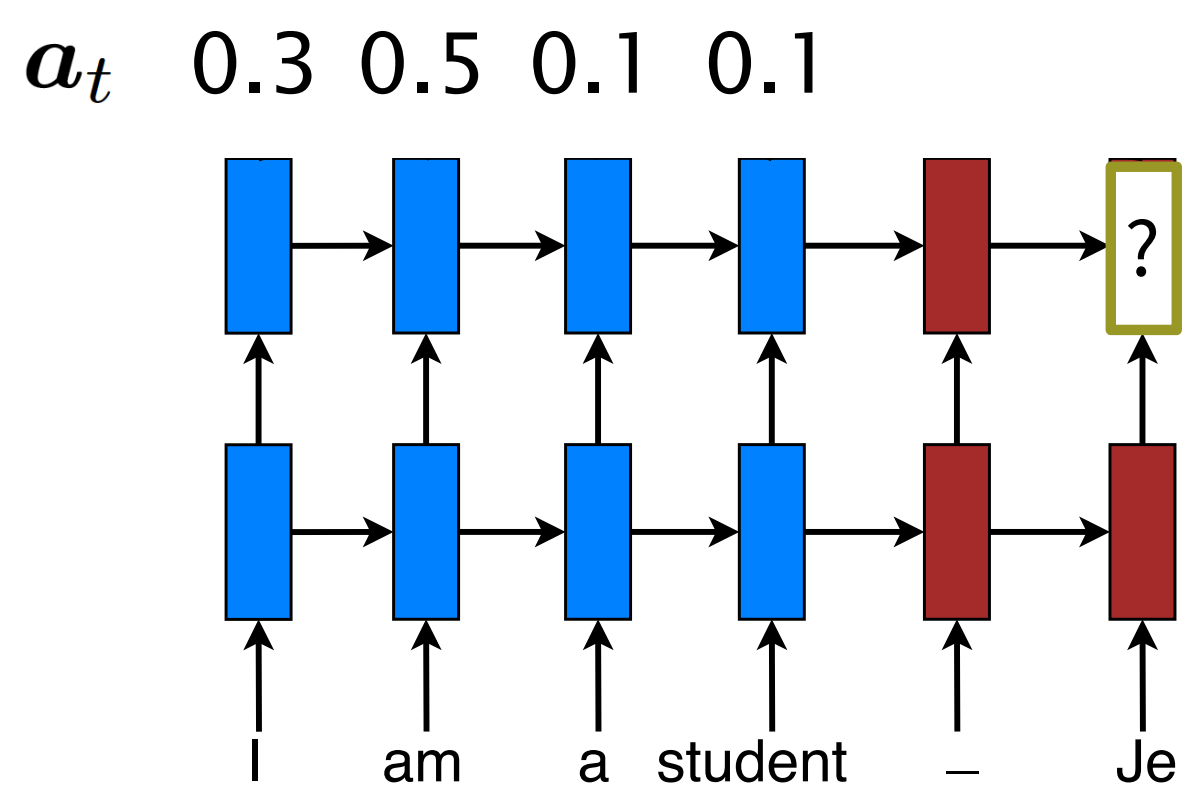

- Convert into alignment weights. 


\section{Attention Mechanism - Context}

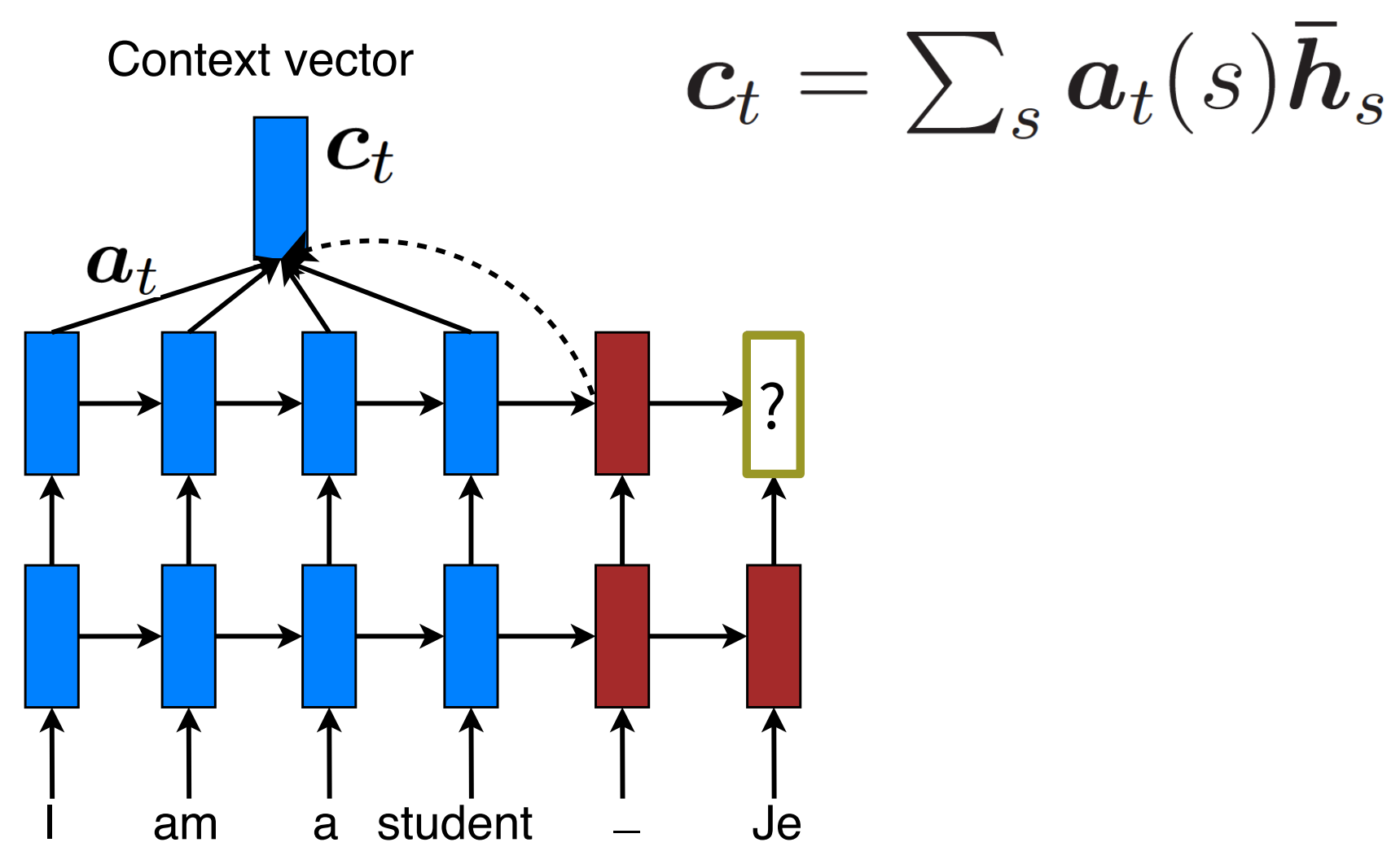

- Build context vector: weighted average. 


\section{Attention Mechanism - Hidden State}

Context vector

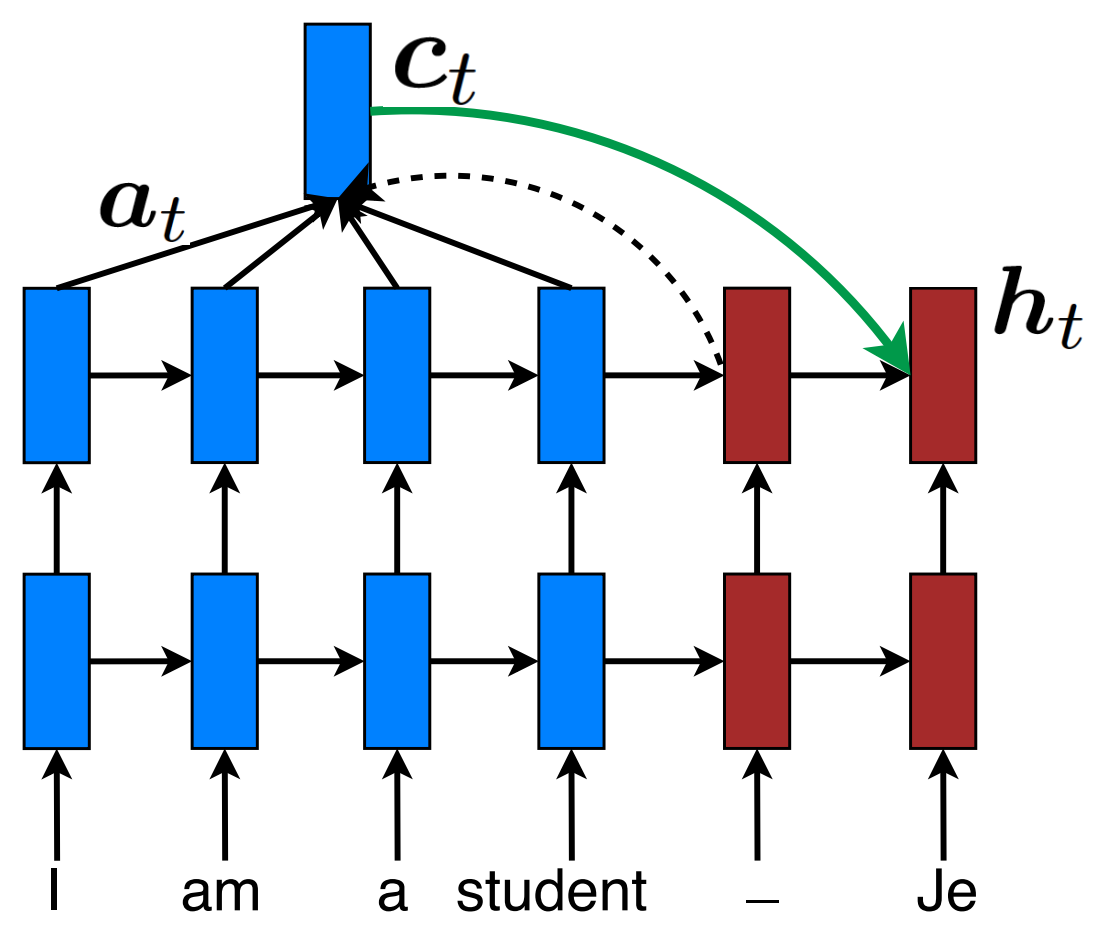

- Compute the next hidden state. 


\section{Attention Mechanisms+}

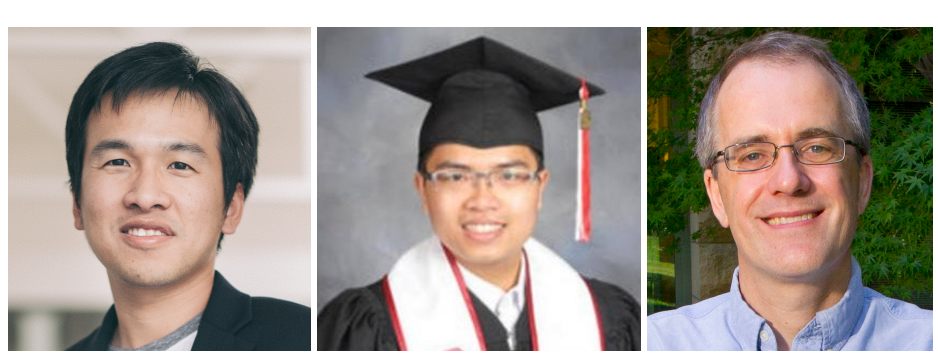

- Simplified mechanism \& more functions:

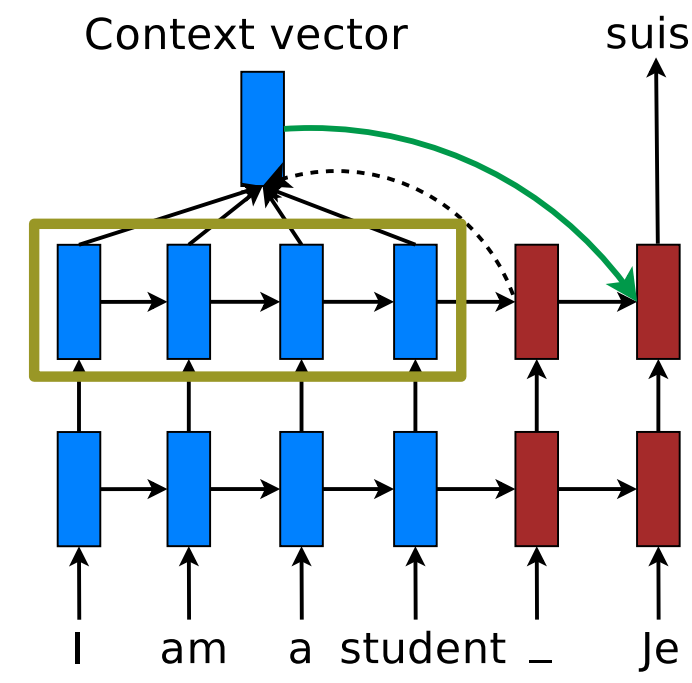

$$
\operatorname{score}\left(\boldsymbol{h}_{t}, \overline{\boldsymbol{h}}_{s}\right)=\left\{\begin{array}{l}
\boldsymbol{h}_{t}^{\top} \overline{\boldsymbol{h}}_{s} \\
\boldsymbol{h}_{t}^{\top} \boldsymbol{W}_{\boldsymbol{a}} \overline{\boldsymbol{h}}_{s} \\
\boldsymbol{v}_{a}^{\top} \tanh \left(\boldsymbol{W}_{\boldsymbol{a}}\left[\boldsymbol{h}_{t} ; \overline{\boldsymbol{h}}_{s}\right]\right)
\end{array}\right.
$$

Thang Luong, Hieu Pham, and Chris Manning. Effective Approaches to Attention-based Neural Machine Translation. EMNLP'15. 


\section{Better Translation of Long Sentences}

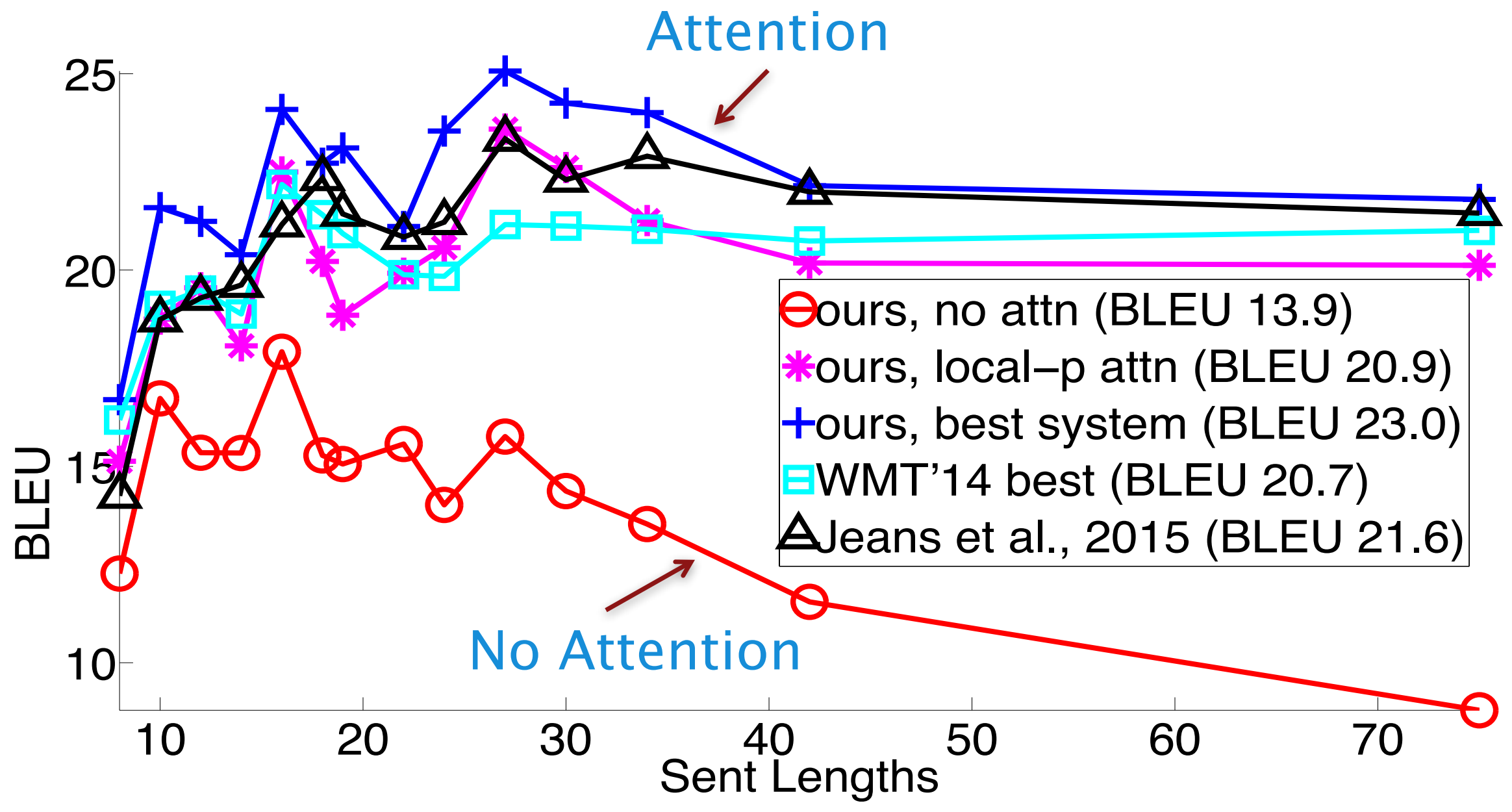




\section{Better Translation of Long Sentences}

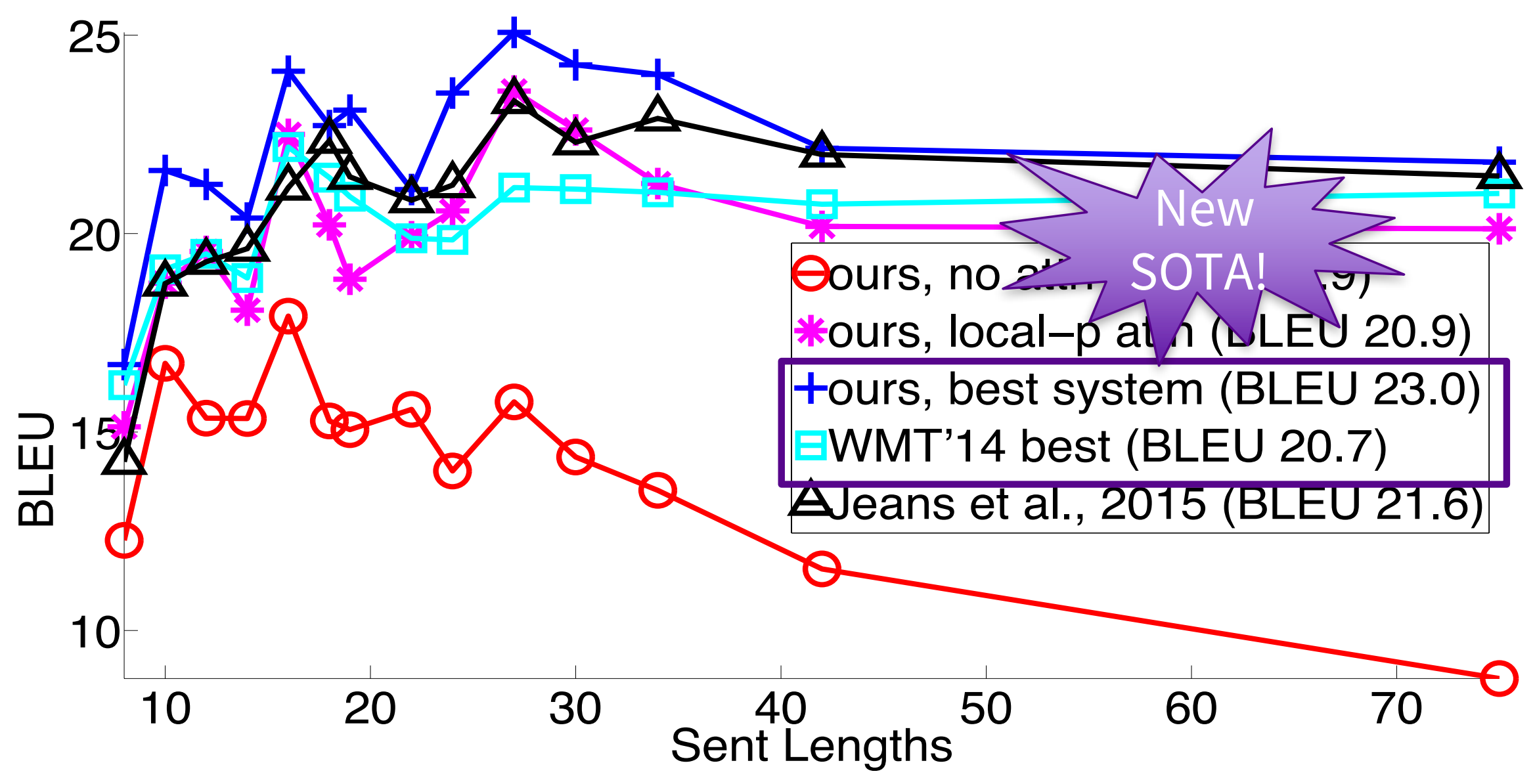




\section{Sample English-German translations}

\begin{tabular}{c|l} 
source & Orlando Bloom and Miranda Kerr still love each other \\
\hline human & Orlando Bloom und Miranda Kerr lieben sich noch immer \\
\hline +attn & $\begin{array}{l}\text { Orlando Bloom und Miranda Kerr } \\
\text { immer. }\end{array}$ \\
\hline base & $\begin{array}{l}\text { Orlando Bloom und Lucas Miranda lieben einander noch } \\
\text { immer. }\end{array}$ \\
- Translates names correctly.
\end{tabular}




\section{Sample English-German translations}

\begin{tabular}{|c|c|}
\hline source & $\begin{array}{l}\text { We ' re pleased the FAA recognizes that an enjoyable passenger experience is } \\
\text { not incompatible with safety and security, said Roger Dow, CEO of the U.S. } \\
\text { Travel Association. }\end{array}$ \\
\hline human & $\begin{array}{l}\text { Wir freuen uns, dass die FAA erkennt, dass ein angenehmes } \\
\text { Passagiererlebnis nicht im Wider- spruch zur Sicherheit steht, sagte Roger } \\
\text { Dow, CEO der U.S. Travel Association. }\end{array}$ \\
\hline+ attn & $\begin{array}{l}\text { Wir freuen uns, dass die FAA anerkennt, dass ein angenehmes ist nicht mit } \\
\text { Sicherheit und Sicherheit unvereinbar ist, sagte Roger Dow, CEO der US - } \\
\text { die. }\end{array}$ \\
\hline base & $\begin{array}{l}\text { Wir freuen uns u'ber die }<\text { unk }>\text {, dass ein }<\text { unk }><\text { unk }>\text { mit Sicherheit nicht } \\
\text { verein bar ist mit Sicherheit und Sicherheit, sagte Roger Cameron, CEO der } \\
\text { US - < unk }>\text {. }\end{array}$ \\
\hline
\end{tabular}

- Translates a doubly-negated phrase correctly. 


\section{Sample English-German translations}

We ' re pleased the FAA recognizes that an enjoyable passenger experience is source not incompatible with safety and security, said Roger Dow , CEO of the U.S. Travel Association .

Wir freuen uns, dass die FAA erkennt, dass ein angenehmes

human Passagiererlebnis nicht im Wider- spruch zur Sicherheit steht, sagte Roger Dow , CEO der U.S. Travel Association .

Wir freuen uns, dass die FAA anerkennt, dass ein angenehmes ist nicht mit +attn Sicherheit und Sicherheit unvereinbar ist, sagte Roger Dow , CEO der US die.

Wir freuen uns u'ber die <unk $>$, dass ein $<$ unk $><$ unk $>$ mit Sicherheit nicht base verein bar ist mit Sicherheit und Sicherheit, sagte Roger Cameron, CEO der US - <unk>

- Translates a doubly-negated phrase correctly. 


\section{More Attention! The idea of coverage}

- Caption generation

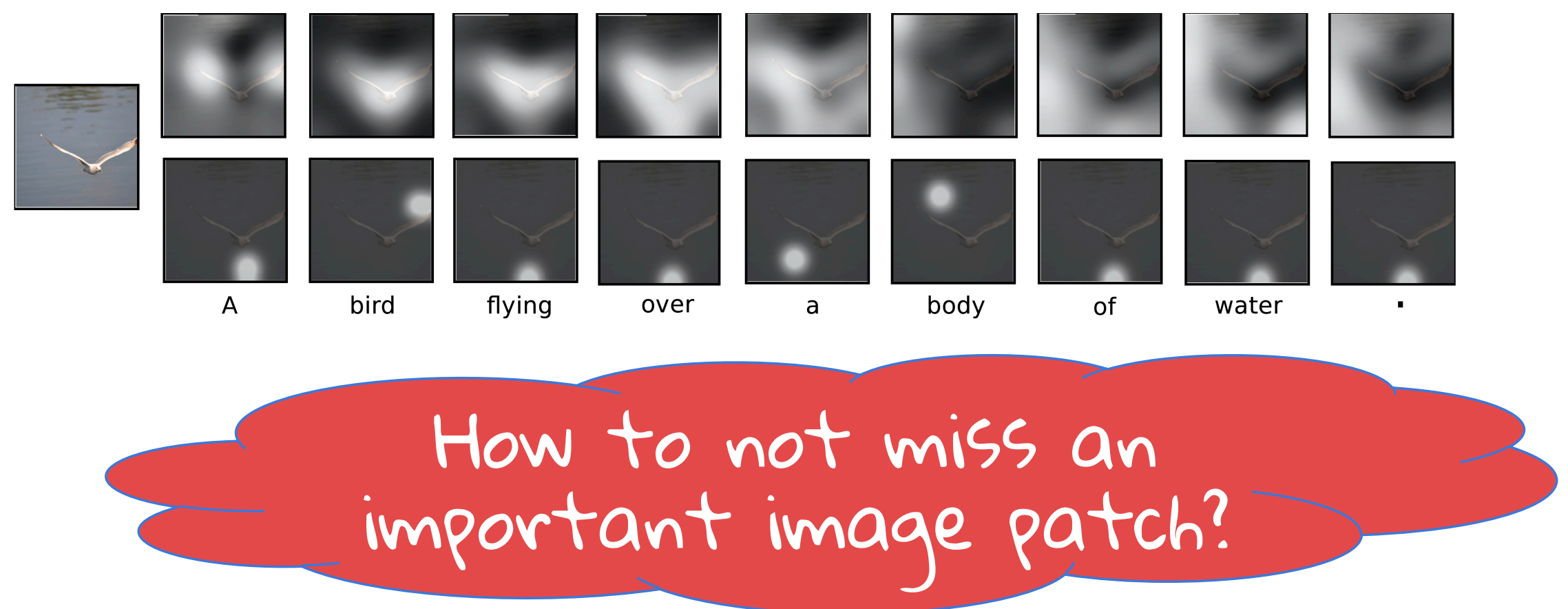

Xu, Ba, Kiros, Cho, Courville, Salakhutdinov, Zemel, Bengio. Show, Attend and Tell: Neural Image Caption Generation with Visual Attention. ICML'15 


\section{Doubly attention $-\log (P(\mathbf{y} \mid \mathbf{x}))+\lambda \sum_{\text {Per image patch }}^{L}\left(1-\sum_{\substack{t \\ k}}^{C} \alpha_{t i}\right)^{2}$ \\ - Sum to 1 in both dimensions caption words}

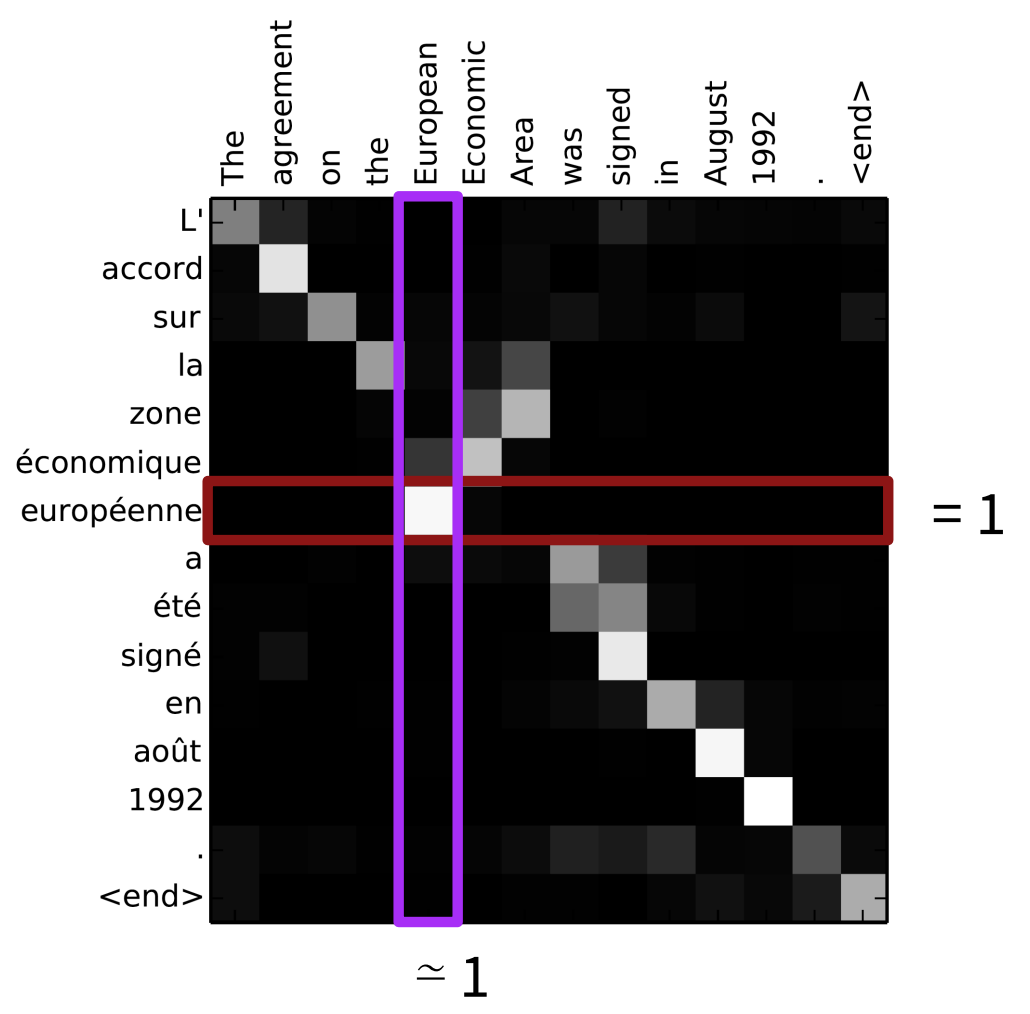

\section{Coverage set exists long time ago in SM T!}

Xu, Ba, Kiros, Cho, Courville, Salakhutdinov, Zemel, Bengio. Show, Attend and Tell: Neural Image Caption Generation with Visual Attention. ICML'15 


\section{Extend to NMT - Linguistic insights}

- [Cohn, Hoang, Vymolova, Yao, Dyer, Haffari, NAACL'16]: position (IBM2) + Markov (HMM) + fertility (IBM3-5) + alignment symmetry (BerkeleyAligner).

$$
-\log (P(\mathbf{y} \mid \mathbf{x}))+\lambda \sum_{\text {Per source word }}^{L}\left(1-\sum_{t}^{C} \alpha_{t i}\right)^{2}
$$

- [Tu, Lu, Liu, Liu, Li, ACL'16]: linguistic \& NN-based coverage models. 


\section{If you feel jetlagged ... see when MT fails}

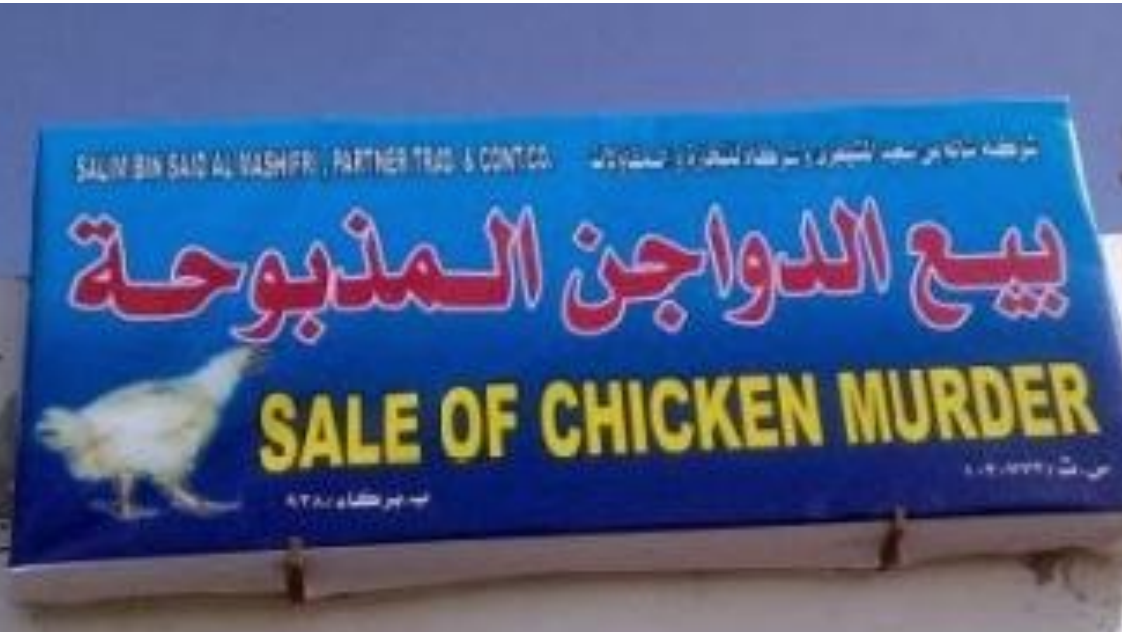

Sale of chicken murder

\section{Kid's menu}

45. Baby wok

Kyckling, wokgrönsaker och ris

46. Deep fried baby

Friterad kyckling sötsursås och ris

47. Baby noodle

Kyckling, wokgrönsaker och äggnudlar

152

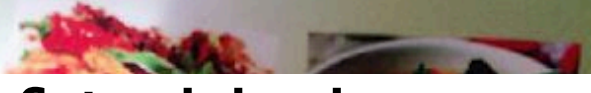

Deep fried baby

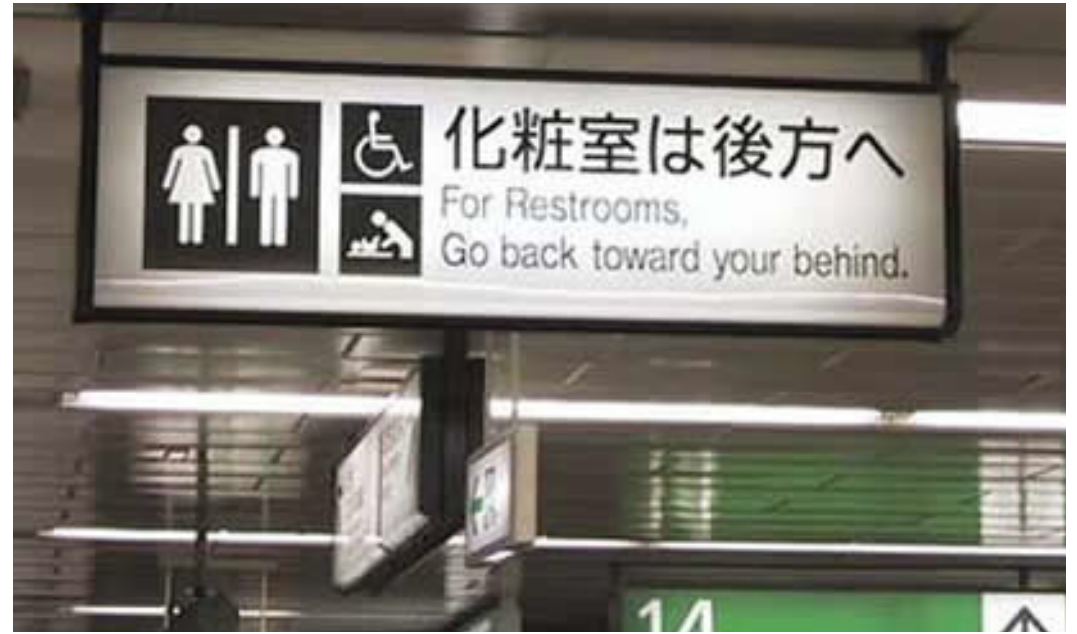

Go back toward your behind

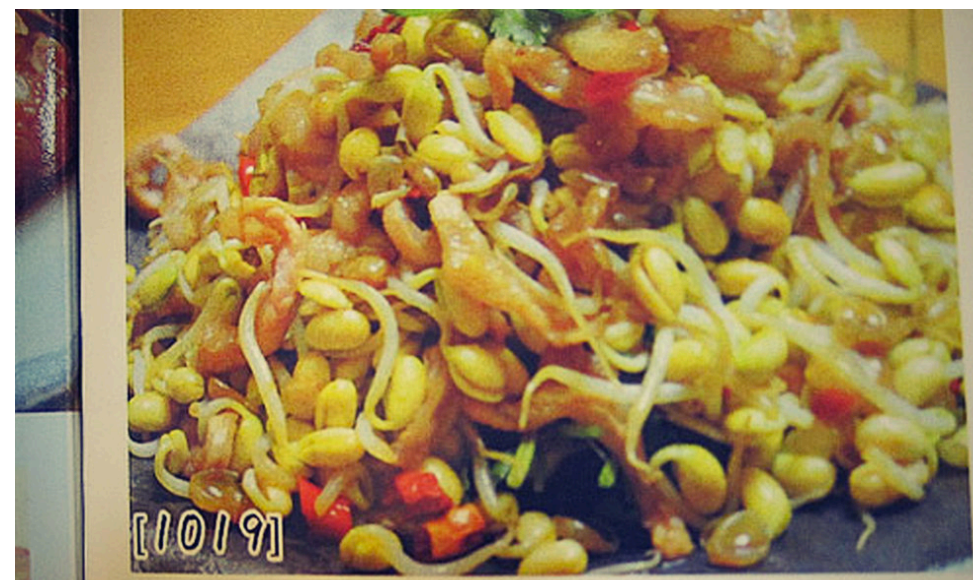

锅叉子肉筋笨豆芽 19元 Meat muscle stupid bean sprouts

Meat muscle stupid bean sprouts 
3. Advancing NMT

a. The vocabulary aspect

- Goal: extend the vocabulary coverage.

b. The memory aspect

- Goal: translate long sentences better.

c. The language complexity aspect

- Goal: handle more language variations.

d. The data aspect

- Goal: utilize more data sources. 


\section{Extend NMT to more languages}

- "Copy" mechanisms are not sufficient.

- Transliteration: Christopher $\mapsto$ Kryštof

- Multi-word alignment: Solar system $\mapsto$ Sonnensystem

- Need to handle large, open vocabulary

- Rich morphology: nejneobhospodařovávatelnějšímu

("to the worst farmable one")

- Informal spelling: goooooood morning !!!!!

\section{Be able to operate at sub-word levels.}




\section{Sub-word modeling}

\section{Again, lots of inspirations \\ from neural language modeling!}




\section{Character-based LSTM}
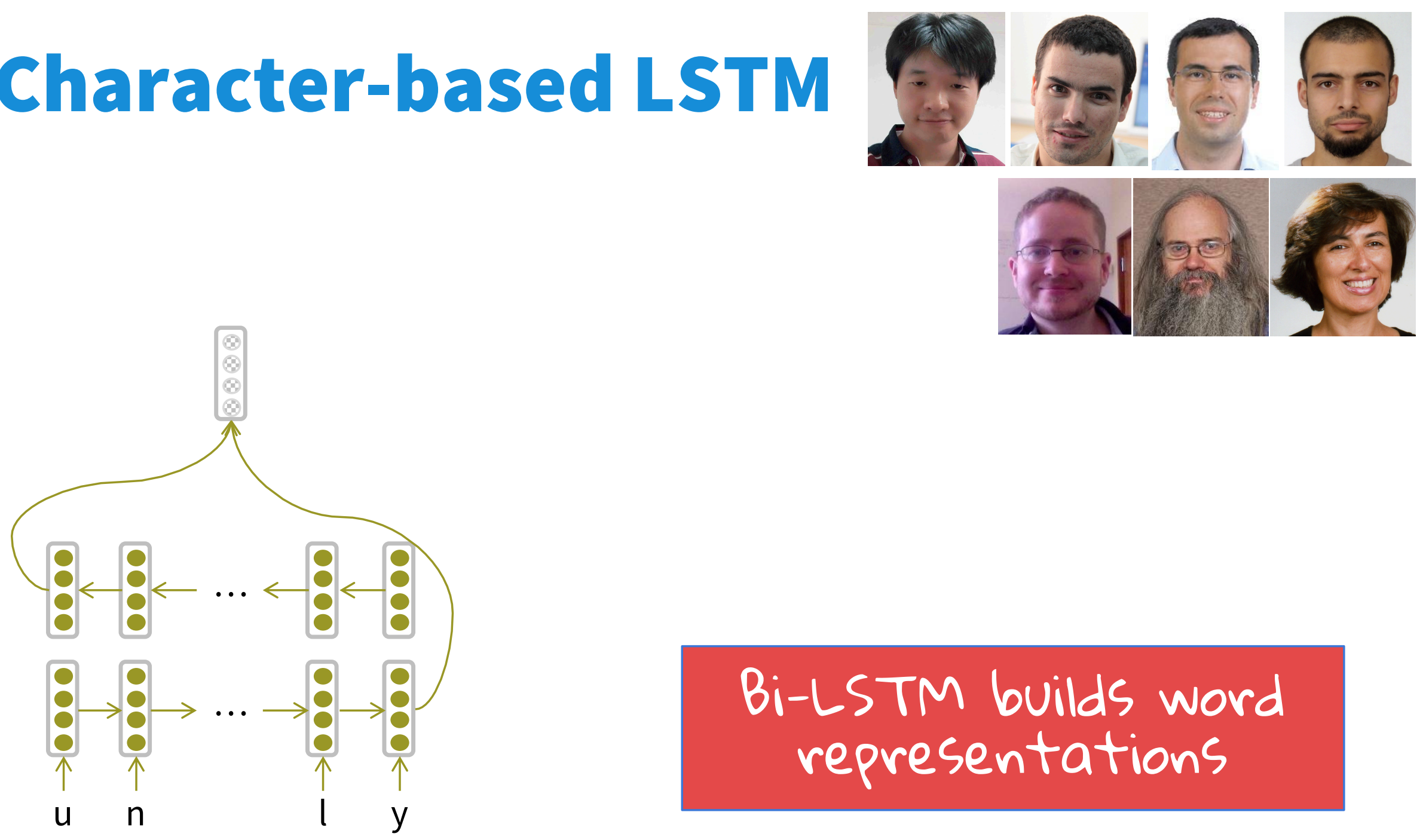

\section{Bi-LSTM builds word representations}

(unfortunately)

Ling, Luís, Marujo, Astudillo, Amir, Dyer, Black, Trancoso. Finding Function in Form: Compositional Character Models for Open Vocabulary Word Representation. EMNLP'15. 156 


\section{Character}

\section{ConvNet}
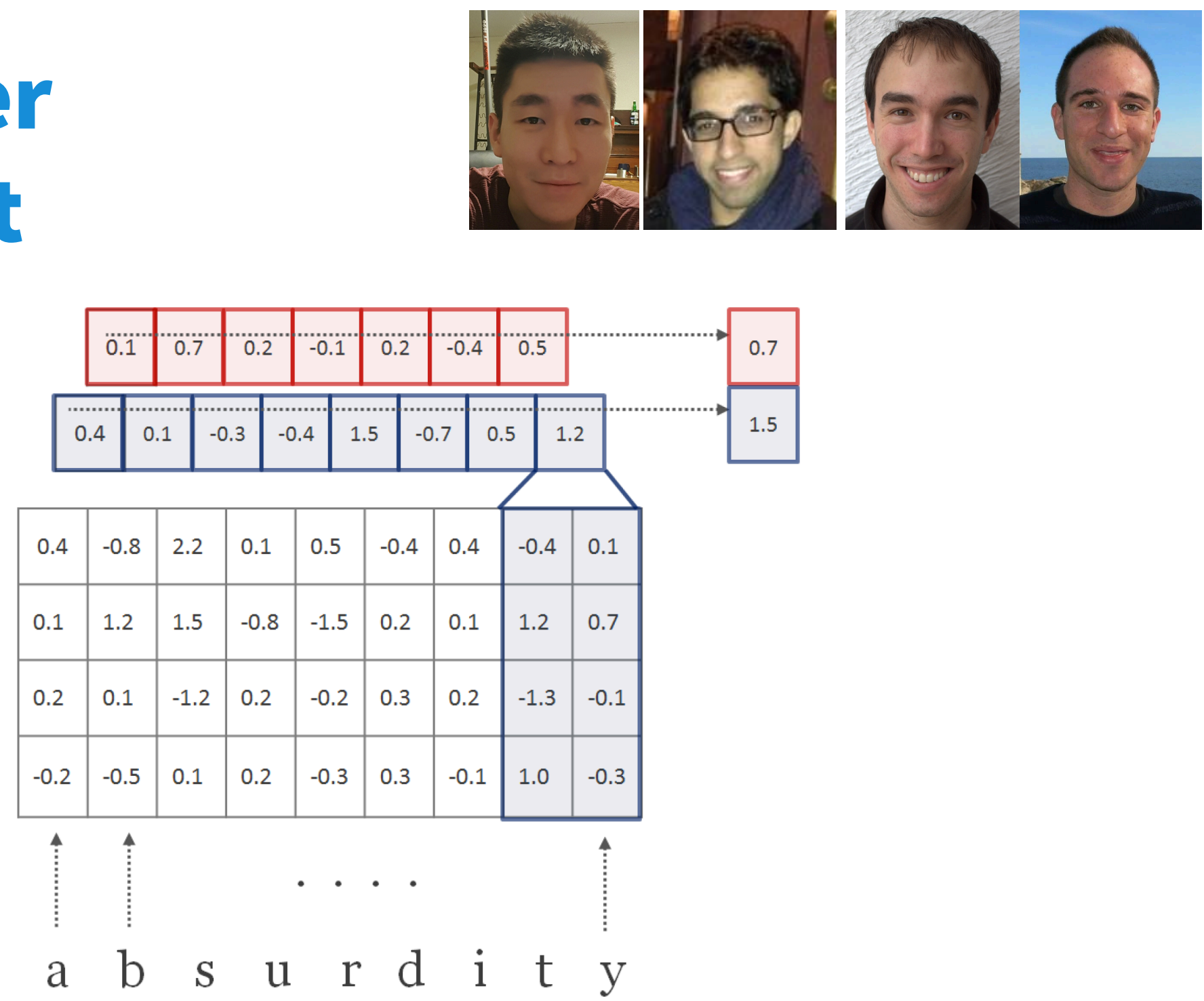

Yoon Kim, Yacine Jernite, David Sontag, and Alexander M. Rush. Character-Aware Neural Language Models. AAAl 2016. 


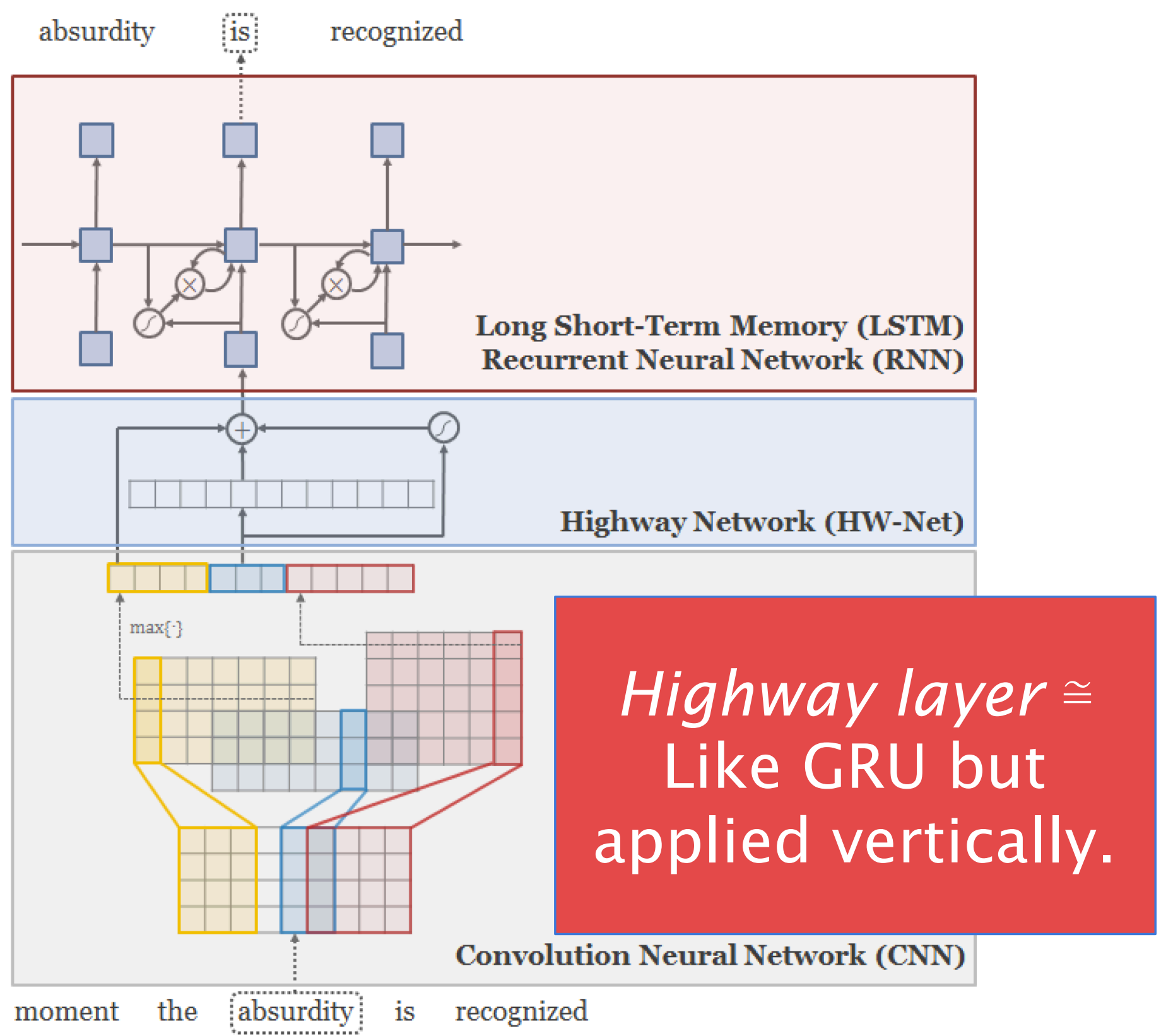




\section{Sub-word NMT: two trends}

- Same seq2seq architecture:

- Use smaller units.

- [Sennrich, Haddow, Birch, ACL'16a], [Chung, Cho, Bengio, ACL'16].

- Hybrid architectures:

- RNN for words + something else for characters.

- [Costa-Jussà \& Fonollosa, ACL'16], [Luong \& Manning, ACL'16]. 


\section{Byte Pair Encoding}

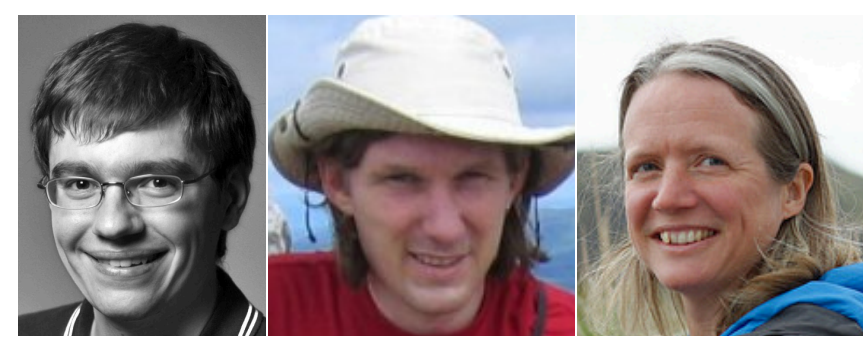

- A compression algorithm:

- Most frequent byte pair $\mapsto$ a new byte.

\section{Replace bytes with character ngrams}

Rico Sennrich, Barry Haddow, and Alexandra Birch. Neural Machine Translation of Rare Words with Subword Units. ACL 2016. 


\section{Byte Pair Encoding}

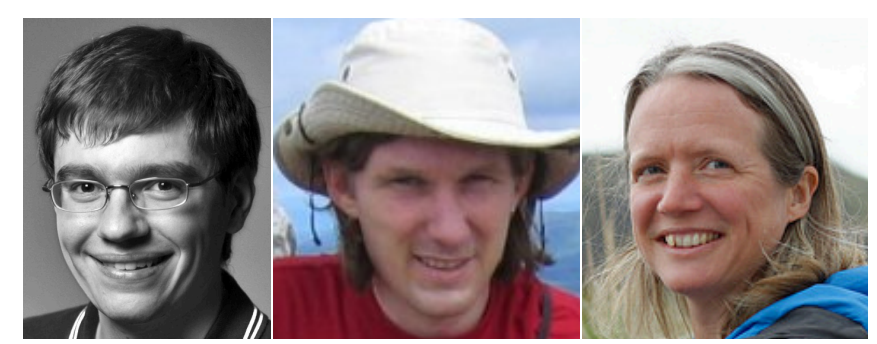

- A word segmentation algorithm:

- Start with a vocabulary of characters.

- Most frequent ngram pairs $\mapsto$ a new ngram. 


\section{Byte Pair Encoding}

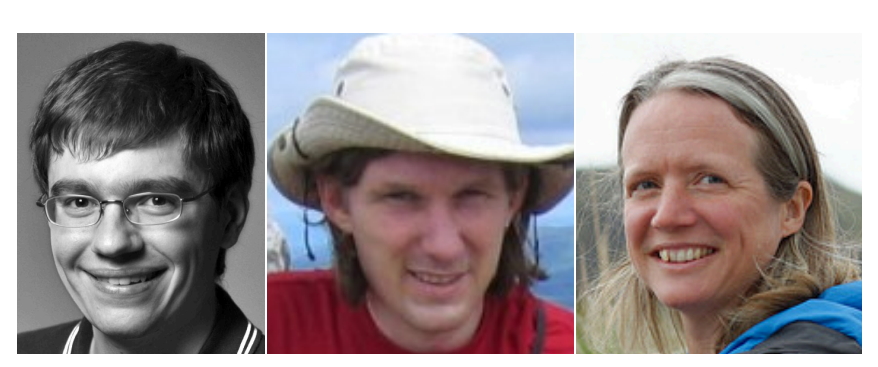

- A word segmentation algorithm:

- Start with a vocabulary of characters.

- Most frequent ngram pairs $\mapsto$ a new ngram.

Dictionary

5 l o w

2 lower

6 n e we st

3 w idest
Vocabulary

I, o, w, e, r, n, w, s, t, i, d

Start with all characters in vocab 


\section{Byte Pair Encoding}

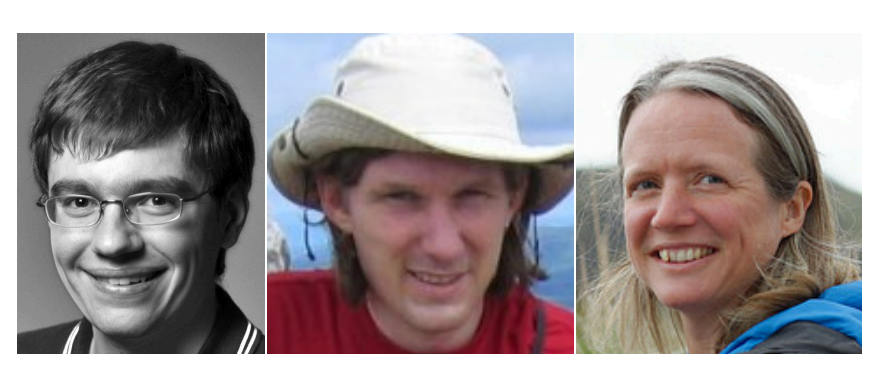

- A word segmentation algorithm:

- Start with a vocabulary of characters.

- Most frequent ngram pairs $\mapsto$ a new ngram.

Dictionary

5 I o w

2 lower

$6 \mathrm{n}$ e w es $\mathrm{t}$

3 wides $t$
Vocabulary

I, o, w, e, r, n, w, s, t, i, d, es

Add a pair $(e, s)$ with frea 9 


\section{Byte Pair Encoding}

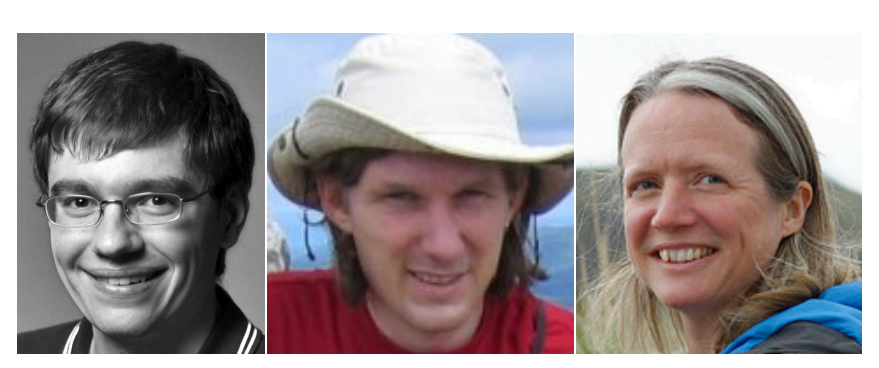

- A word segmentation algorithm:

- Start with a vocabulary of characters.

- Most frequent ngram pairs $\mapsto$ a new ngram.

Dictionary

5 l o w

2 lower

$6 \mathrm{n}$ e w est

3 w i d est
Vocabulary

I, o, w, e, r, n, w, s, t, i, d, es, est

Add a pair (es, t) with frea 9 


\section{Byte Pair Encoding}

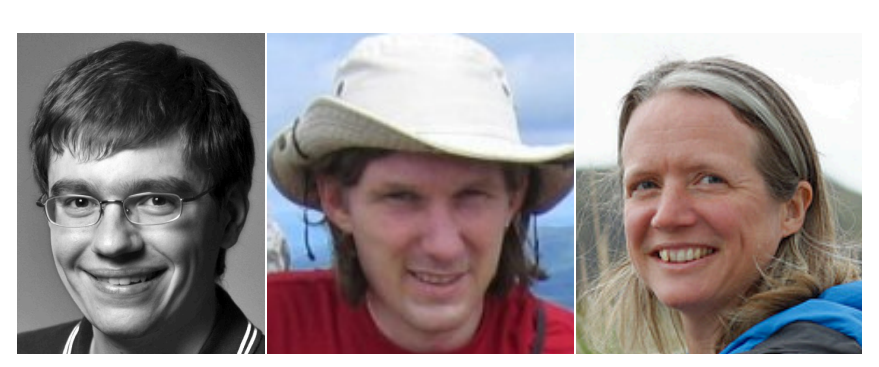

- A word segmentation algorithm:

- Start with a vocabulary of characters.

- Most frequent ngram pairs $\mapsto$ a new ngram.

Dictionary

5 lo $w$

2 lo w e r

$6 \mathrm{n}$ e w est

3 w i d est
Vocabulary

I, o, w, e, r, n, w, s, t, i, d, es, est, lo

Add a pair $(1,0)$ with frea 7 


\section{Byte Pair Encoding}

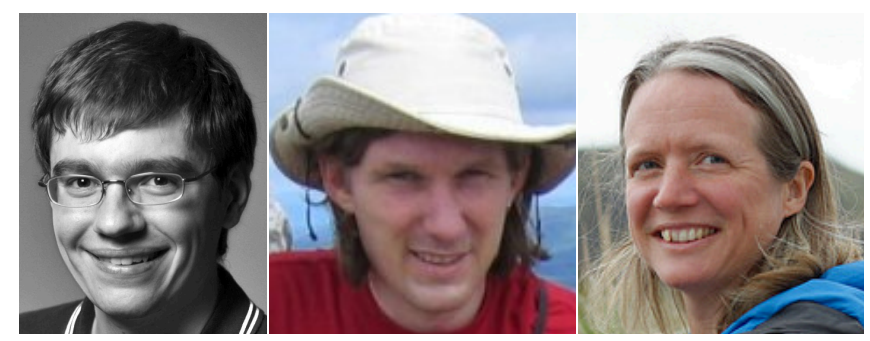

- A word segmentation algorithm:

- Start with a vocabulary of characters.

- Most frequent ngram pairs $\mapsto$ a new ngram.

- Automatically decide vocabs for NMT

-Word-level: asinine situation $\mapsto$ Asinin-Situation

- BPE-level: as in ine situation $\mapsto$ As in in- Situation

\section{Top places in WMT 2Olb!}




\section{BPE $\mapsto$ Characters}

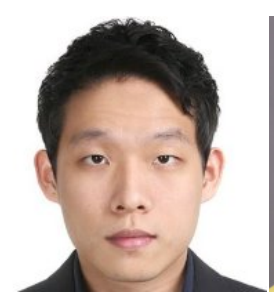

Université In de Montréal

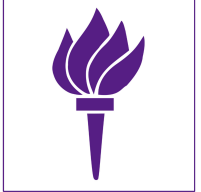

B $\square$ о и

Sequential Processing:

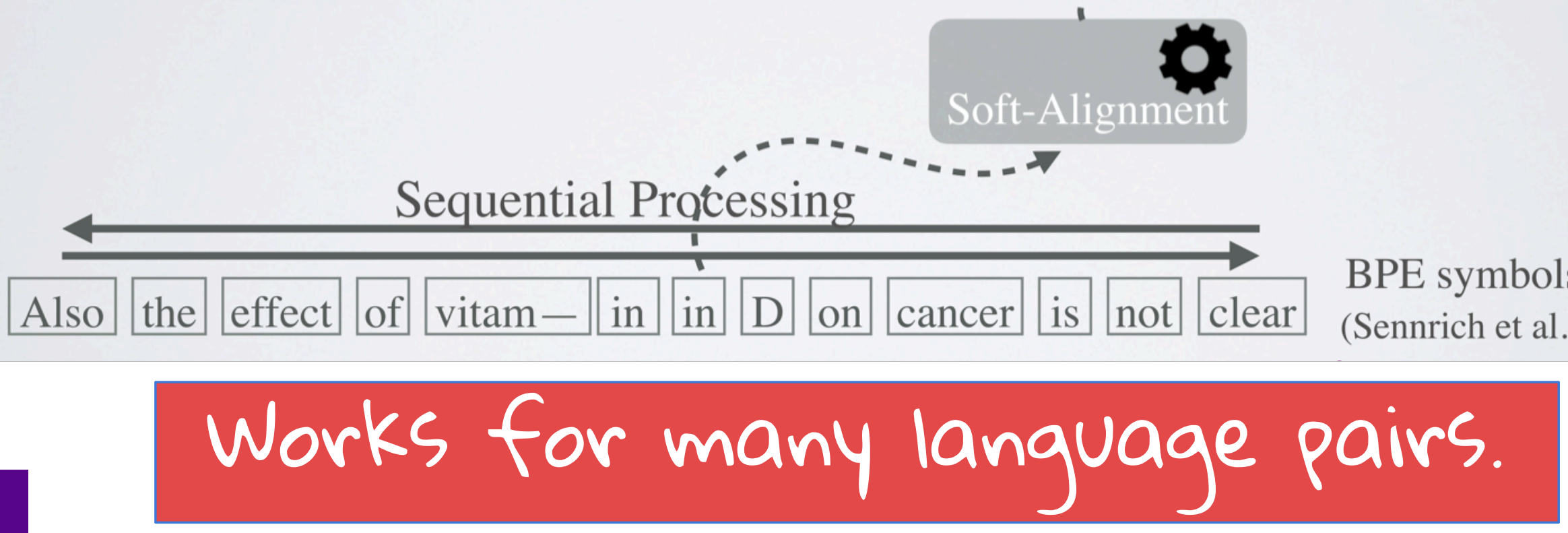

Junyoung Chung, Kyunghyun Cho, Yoshua Bengio. A Character-Level Decoder without Explicit Segmentation for Neural Machine Translation. ACL 2016. 


\section{Sub-word NMT: two trends}

- Same seq2seq architecture:

- Use smaller units.

-(Sennrich et al., ACL'16), (Chung et al., ACL'16).

- Hybrid architectures:

- RNN for words + something else for characters.

- [Costa-Jussà \& Fonollosa, ACL'16], [Luong \& Manning, ACL'16]. 


\section{Character-level Encoder}

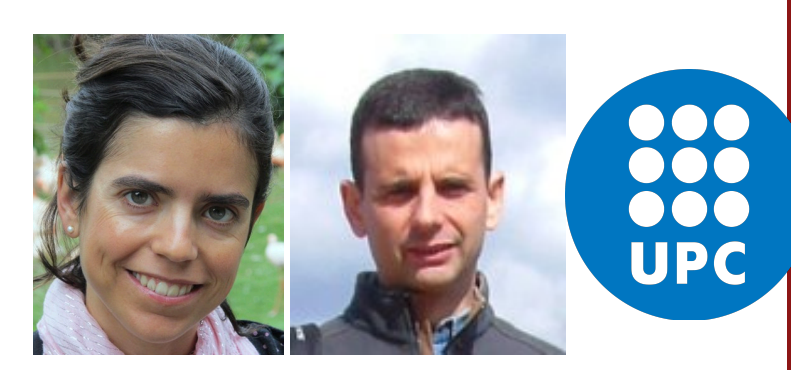

- Useful when source language is complex:

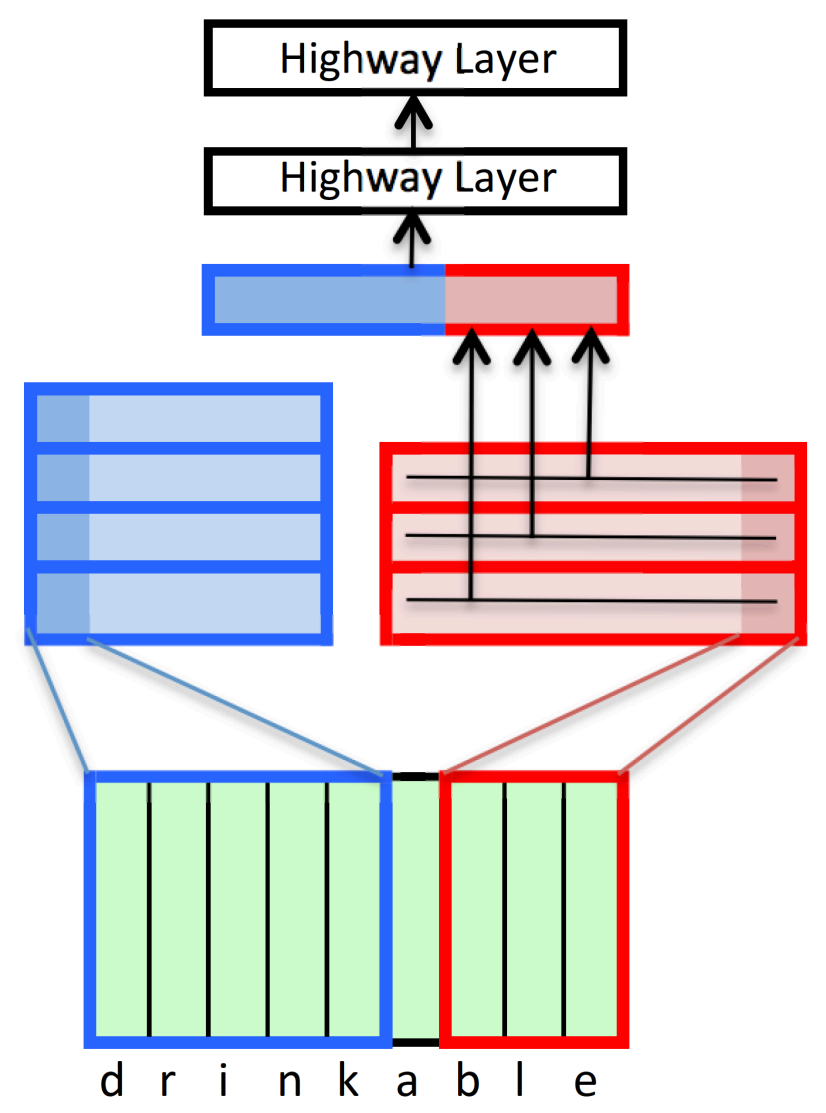

Highway Layers

Max output of

- Similar architecture [Kim, Jernite, Sontag, Rush, AAAl'15].

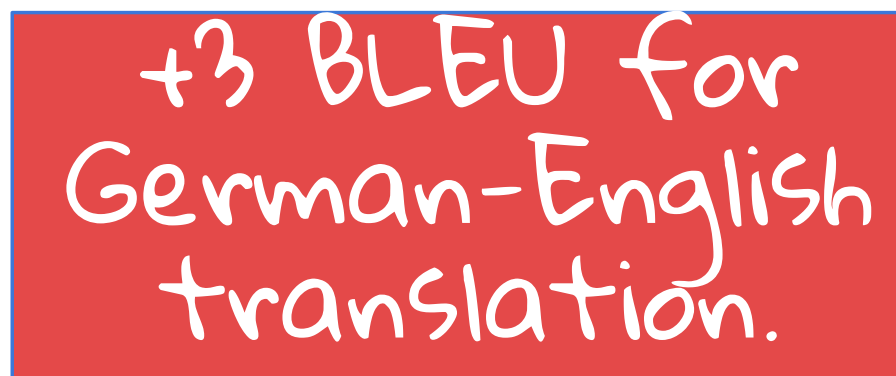

+3 BLEU for translation.

each filter

Multiple

convolution

filters of different

lenghts

Sequence of character

embeddings

Marta R. Costa-jussà and José A. R. Fonollosa. Character-based Neural Machine Translation. ACL'16. 


\section{Hybrid NMT}

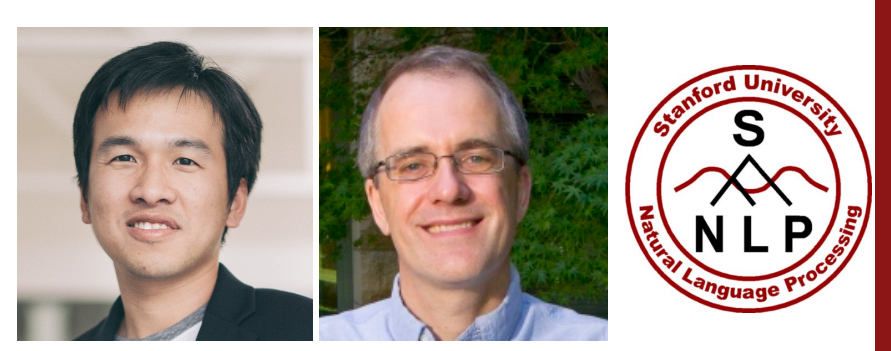

- A best-of-both-worlds architecture:

- Translate mostly at the word level

- Only go the character level when needed.

- More than 2 BLEU improvement over copy mechanism.

Thang Luong and Chris Manning. Achieving Open Vocabulary Neural Machine Translation with Hybrid Word-Character Models. ACL 2016. 


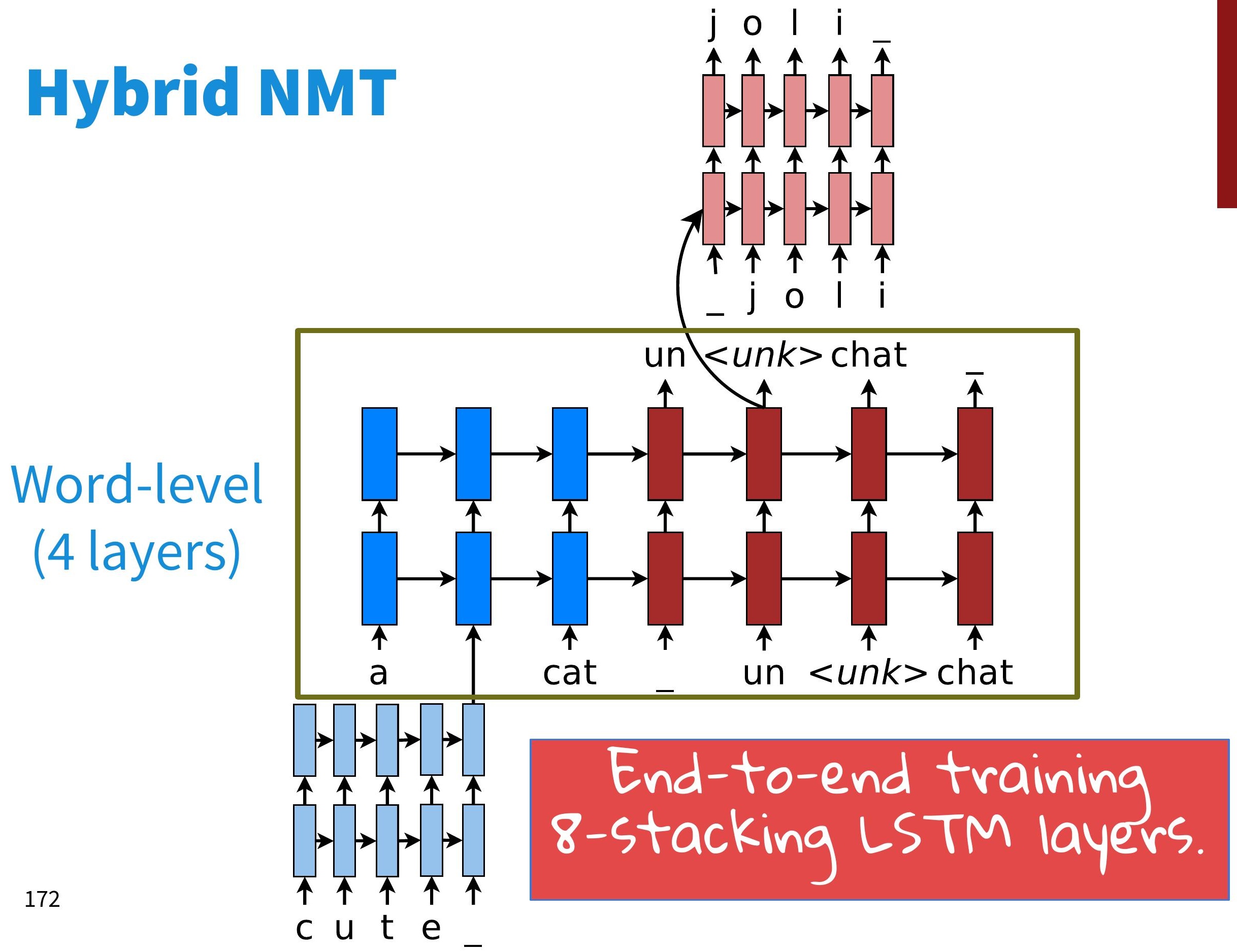




\section{2-stage Decoding}

- Word-level beam search

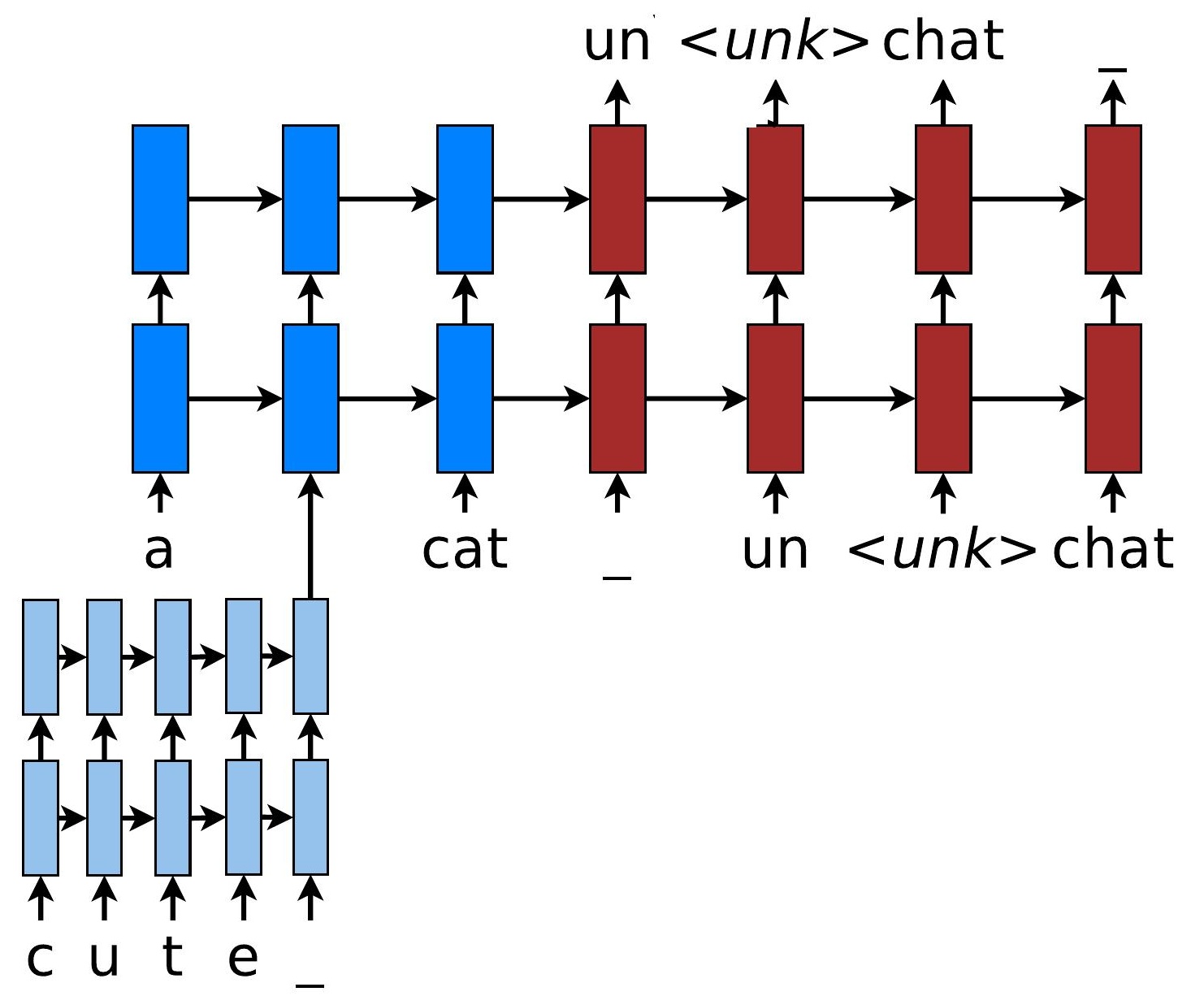




\section{2-stage Decoding}

- Word-level beam search

- Char-level beam search for <unk>.

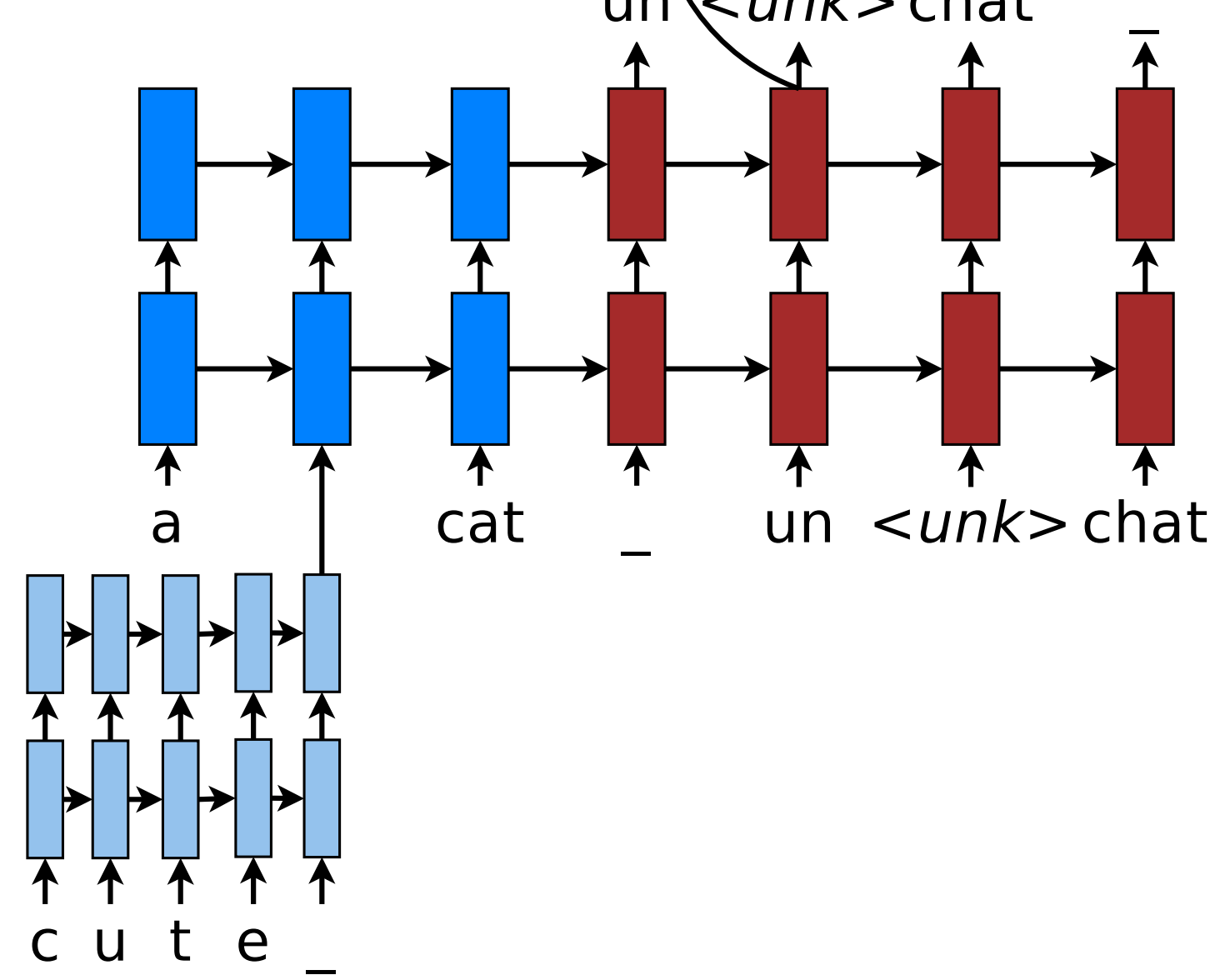




\section{English-Czech Results}

- Train on WMT'15 data (12M sentence pairs)

- newstest2015

\section{Systems}

Winning WMT'15 (Bojar \& Tamchyna, 2015) 18.8

Word-level NMT (Jean et al., 2015)

\section{BLEU}

30x data

3 systems

18.3
Large vocab

+ copy mechanism 


\section{English-Czech Results}

- Train on WMT'15 data (12M sentence pairs)

- newstest2015

\section{Systems}

Winning WMT'15 (Bojar \& Tamchyna, 2015) 18.8

Word-level NMT (Jean et al., 2015)

Hybrid NMT (Luong \& Manning, 2016)*
BLEU

18.3

20.7 30x data

3 systems

Large vocab

+ copy mechanism

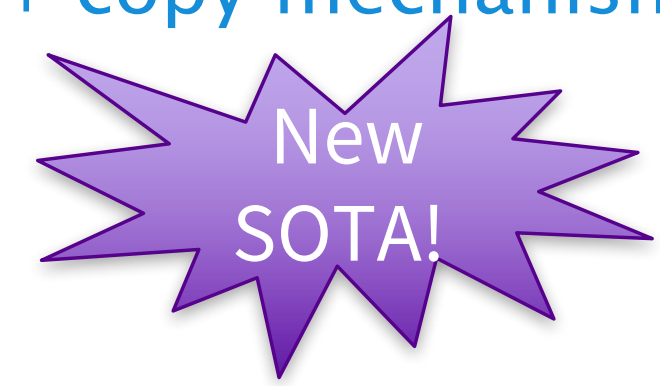




\section{Effects of Vocabulary Sizes}

$\leadsto$ Word $\quad \square$-Word + copy mechanism $\quad-\Delta$ Hybrid

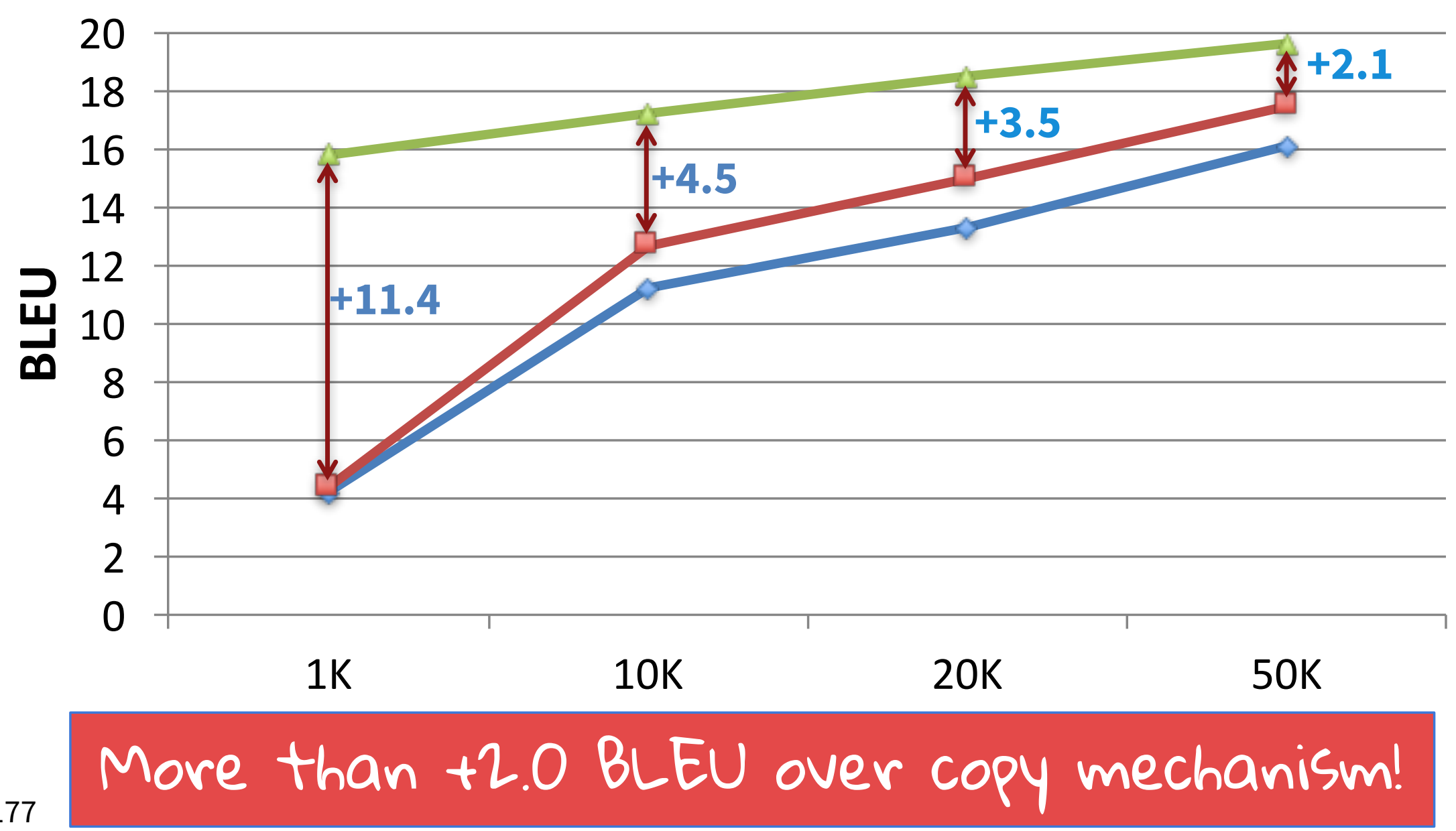




\section{Rare Word Embeddings}

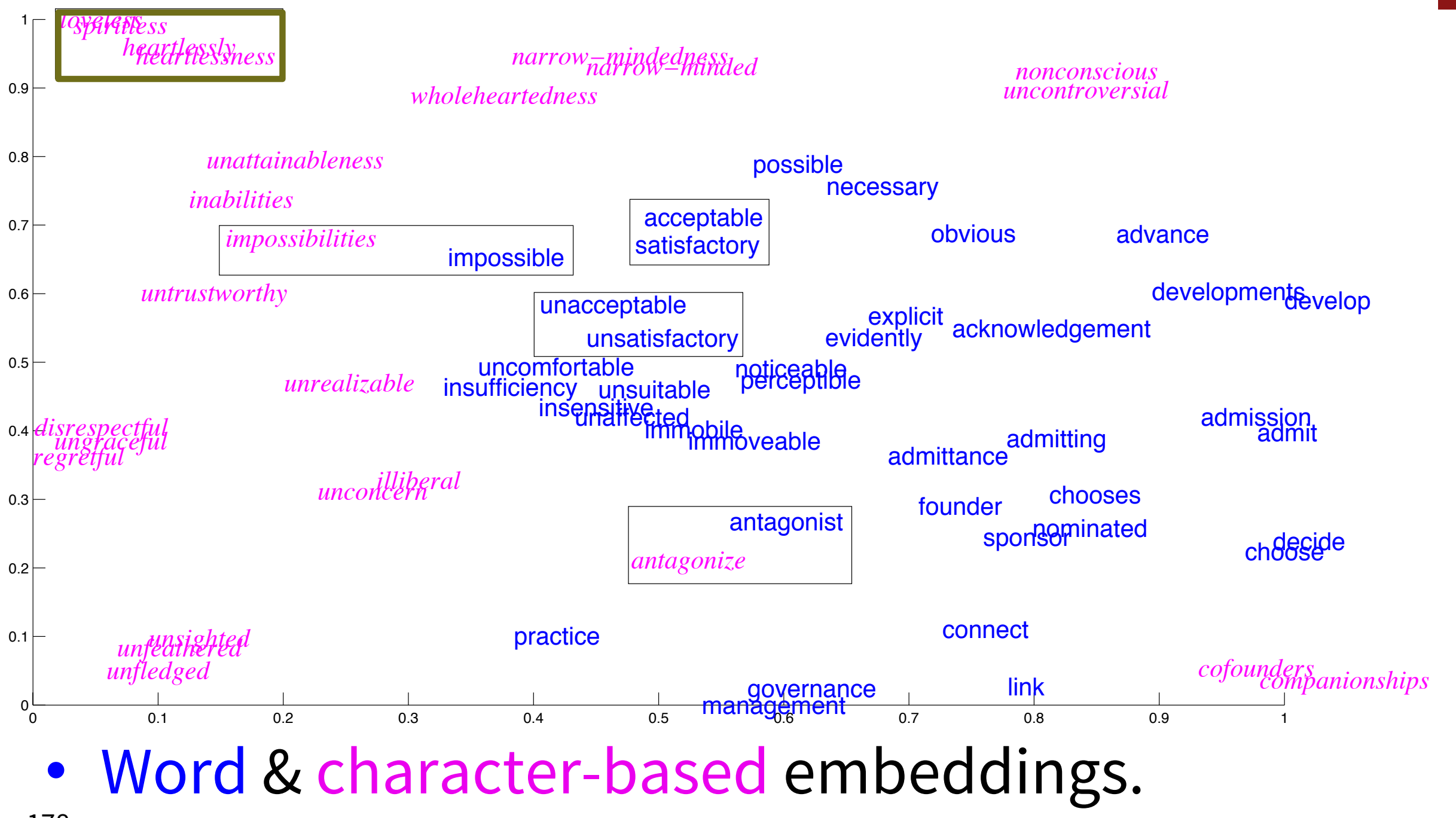




\section{Rare Word Embeddings}

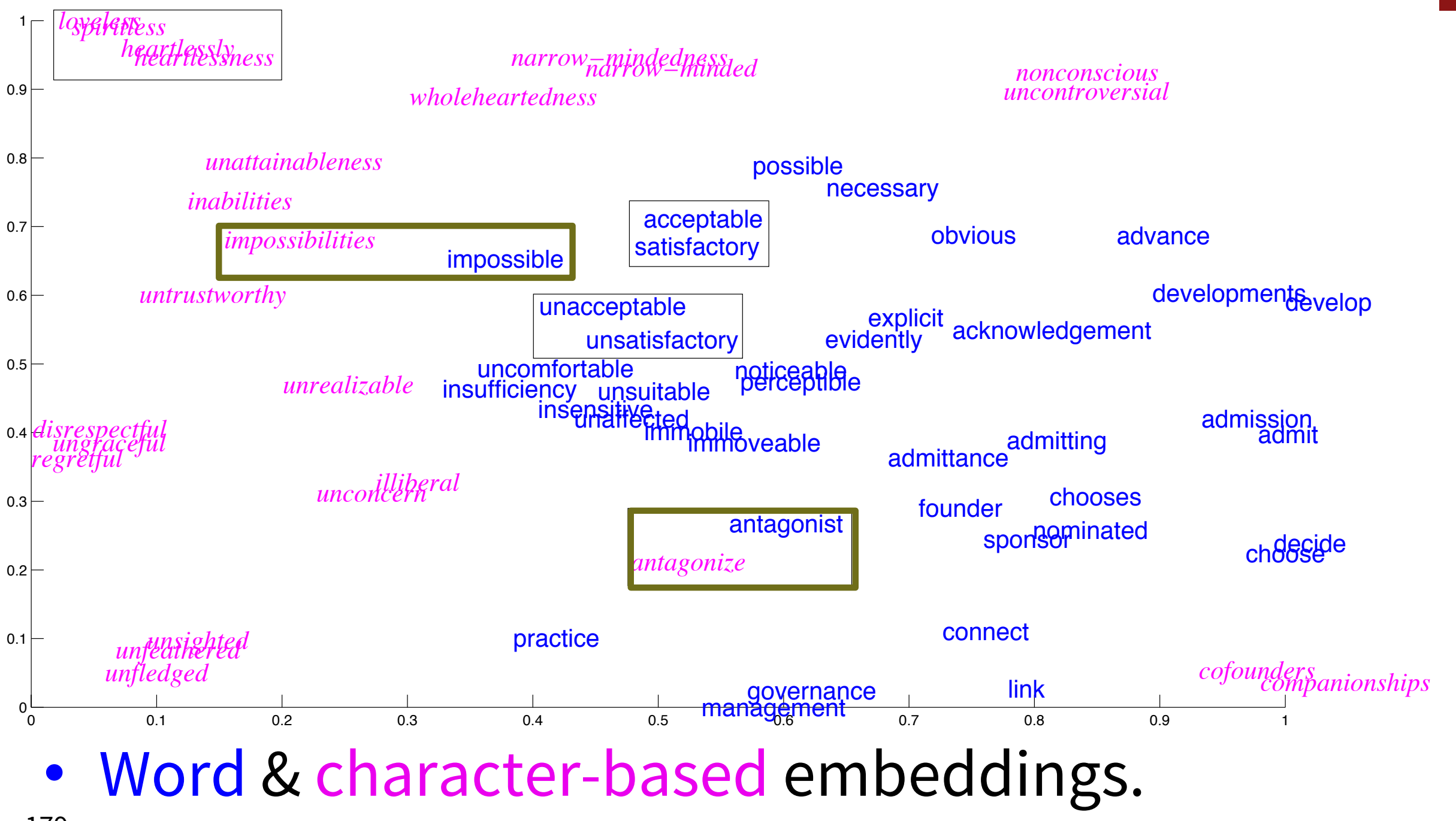




\section{Sample English-Czech translations}

source Her 11-year-old daughter, Shani Bart, said it felt a little bit weird Jeji|jedenáctilletá dcera Shani Bartová prozradila , že je to trochu zvlastni

Její <unk> dcera <unk > <unk> řekla, že je to trochu divné

Její 11-year-old dcera Shani , řekla , že je to trochu divné

Její <unk> dcera, <unk><unk>, řekla, že je to <unk><unk>

hybrid

Jejiljedenáctiletä dcera , Graham Bart, řekla , že cítí trochu divný

- Hybrid: correct, 11-year-old - jedenáctiletá. 


\section{Sample English-Czech translations}

source Her 11-year-old daughter, Shami Bart, said it felt a little bit weird

Její jedenáctiletá dcera Shani Bartová prozradila, že je to trochu zvláštní

Její <unk> dcera <unk > <unk> řekla, že je to trochu divné

Jeji 11-year-old dicera Shani, řekla, že je to trochu divné

Její <unk> dcera, <unk><unk>, řekla, že je to <unk><unk>

hybrid

Její jedenáctiletá dcera, Graham Bart, řekla , že cítí trochu divný

- Word-based: identity copy fails. 
3. Advancing NMT

a. The vocabulary aspect

- Goal: extend the vocabulary coverage.

b. The memory aspect

- Goal: translate long sentences better.

c. The language complexity aspect

- Goal: handle more language variations.

d. The data aspect

- Goal: utilize more data sources. 


\section{Can we utilize other data sources?}

- Multi-lingual: learn from many language pairs?

- SMT-inspired: utilize monolingual data?

- Multi-task: combine seq2seq tasks?
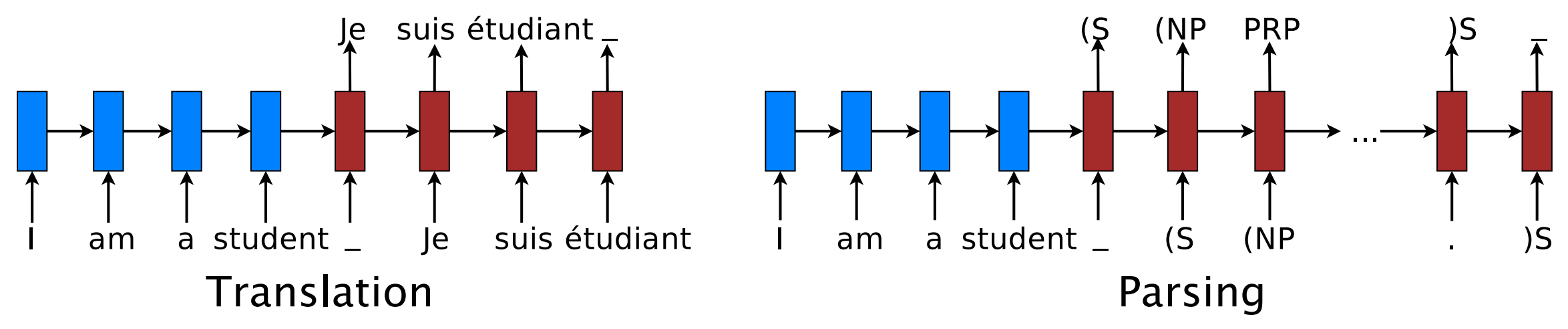


\section{Can we utilize other data sources?}

- Multi-lingual: learn from many language pairs?

- SMT-inspired: utilize monolingual data?

- Multi-task: combine seq2seq tasks?

More later by Cho! 


\section{Integrating Language Models}

- Score interpolation:

Language model scores

$\log p\left(\mathbf{y}_{t}=k\right)=\log p_{\mathrm{TM}}\left(\mathbf{y}_{t}=k\right)+\beta \log p_{\mathrm{LM}}\left(\mathbf{y}_{t}=k\right)$

Hyperparameter

- Deep fusion: combine hidden states instead.

- Controller learns interpolation weights.

- Better than shallow score interpolation.

\section{Improve low-resource language pairs}

Gulcehre, Firat, Xu, Cho, Barrault, Lin, Bougares, Schwenk, Bengio. ${ }^{185}$ On Using Monolingual Corpora in Neural Machine Translation. arXiv 2015. 


\section{Autoencoders}

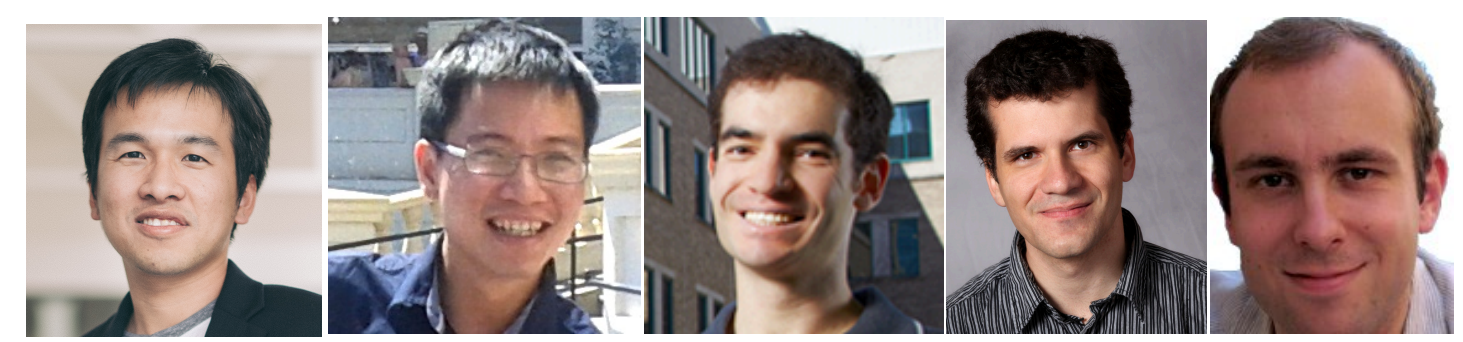

- Shared encoders \& decoders: 3 tasks

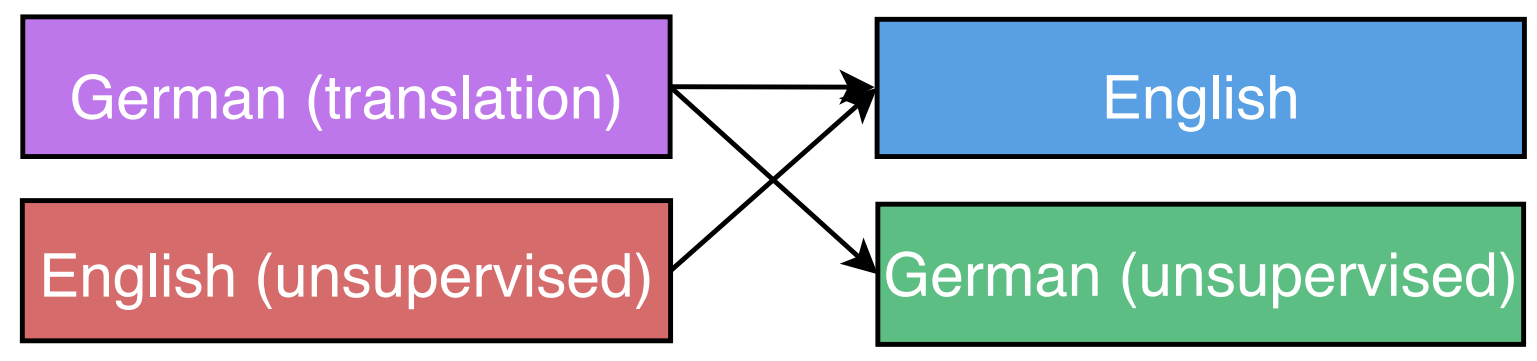

- Small amount of mono data as regularization.

$\bullet+0.9$ BLEU improvements

\section{How to utilize more monolingual data?}

Thang Luong, Quoc Le, Ilya Sutskever, Oriol Vinyals, Lukasz Kaiser. Multi-task sequence to sequence learning. ICLR 2016. 


\section{Enriching parallel data}

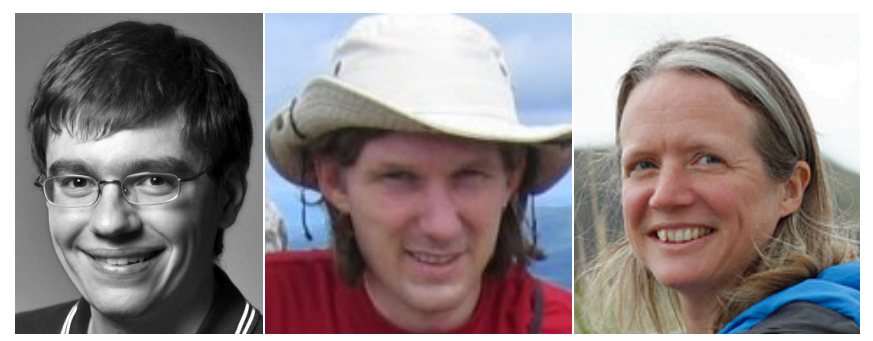

- Dummy source sentences

She loves cute cats

$<$ null $>$
Elle aime les chats mignons

(parallel)

Elle aime les chiens mignons

\section{Small gain +0.4-1.0 BLEU. Difficult to add more mono data.}

Rico Sennrich, Barry Haddow, and Alexandra Birch. Improving Neural Machine Translation Models with Monolingual Data. ACL 2016. 


\section{Enriching parallel data}

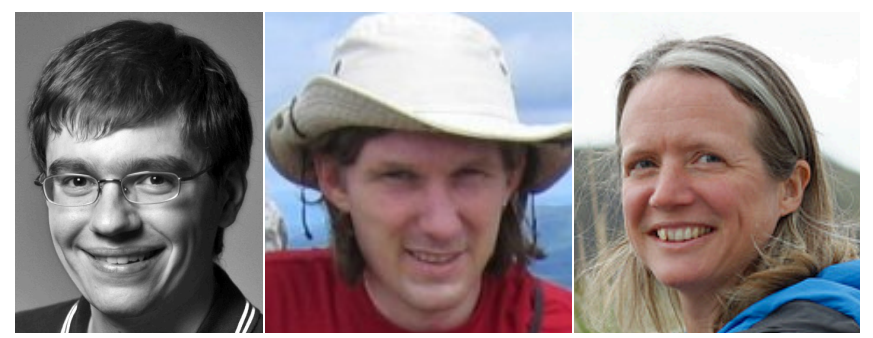

- Synthetic source sentences

She loves cute cats

She likes cute cats

\section{Elle aime les chats mignons}

Elle aime les chiens mignons (parallel)

(mono)

\section{Back translated}

\section{Large gain +2.1-3.4 BLEU.}

Rico Sennrich, Barry Haddow, and Alexandra Birch. Improving Neural Machine Translation Models with Monolingual Data. ACL 2016. 


\section{Prevent Over-fitting}

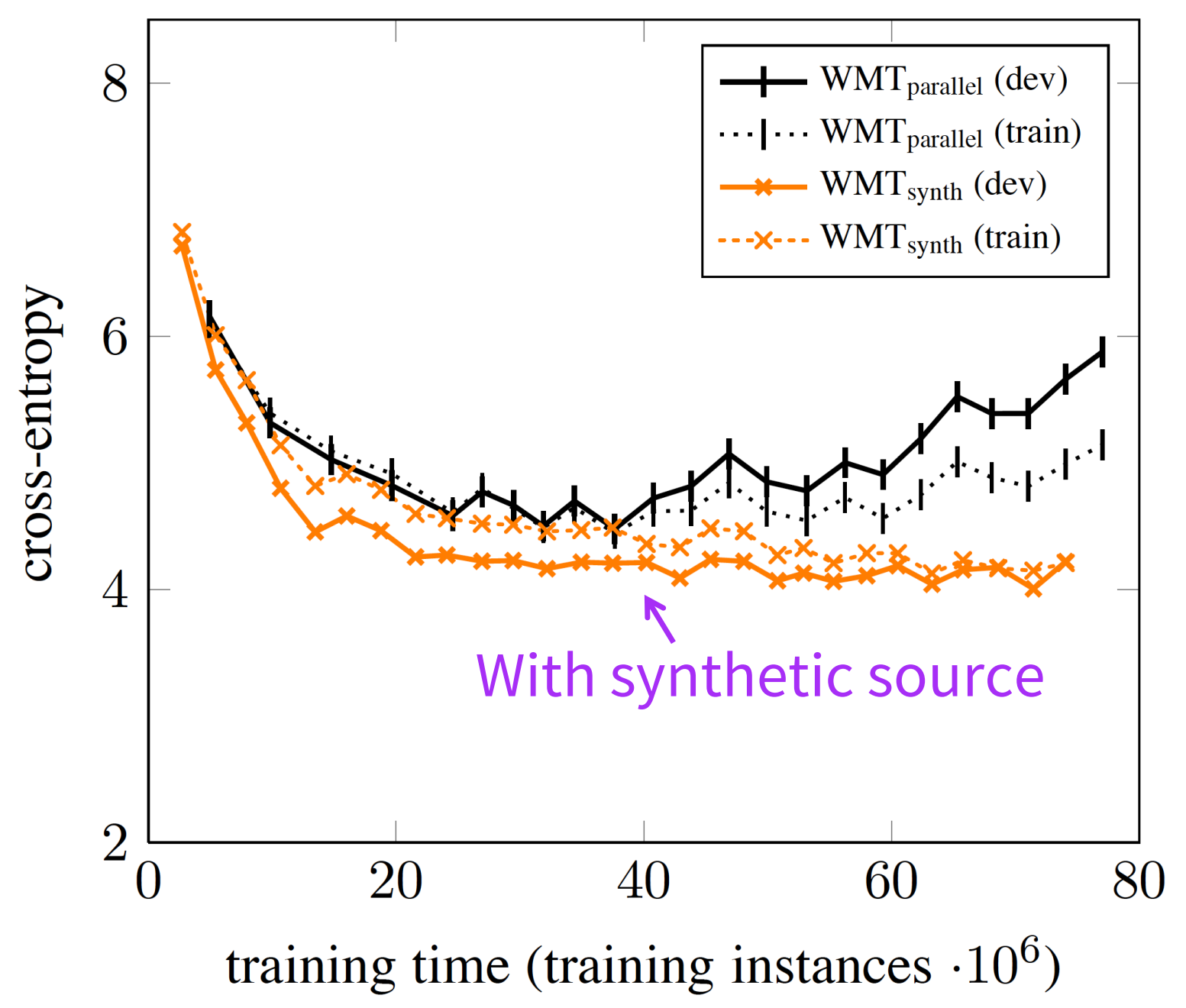




\section{Future of NMT}

a. Multi-task learning

b. Larger context

c. Mobile devices

d. Beyond Maximum Likelihood Estimation 


\section{Future of NMT}

a. Multi-task learning

b. Larger context

c. Mobile devices

d. Beyond Maximum Likelihood Estimation 


\section{Multilingual Translation}

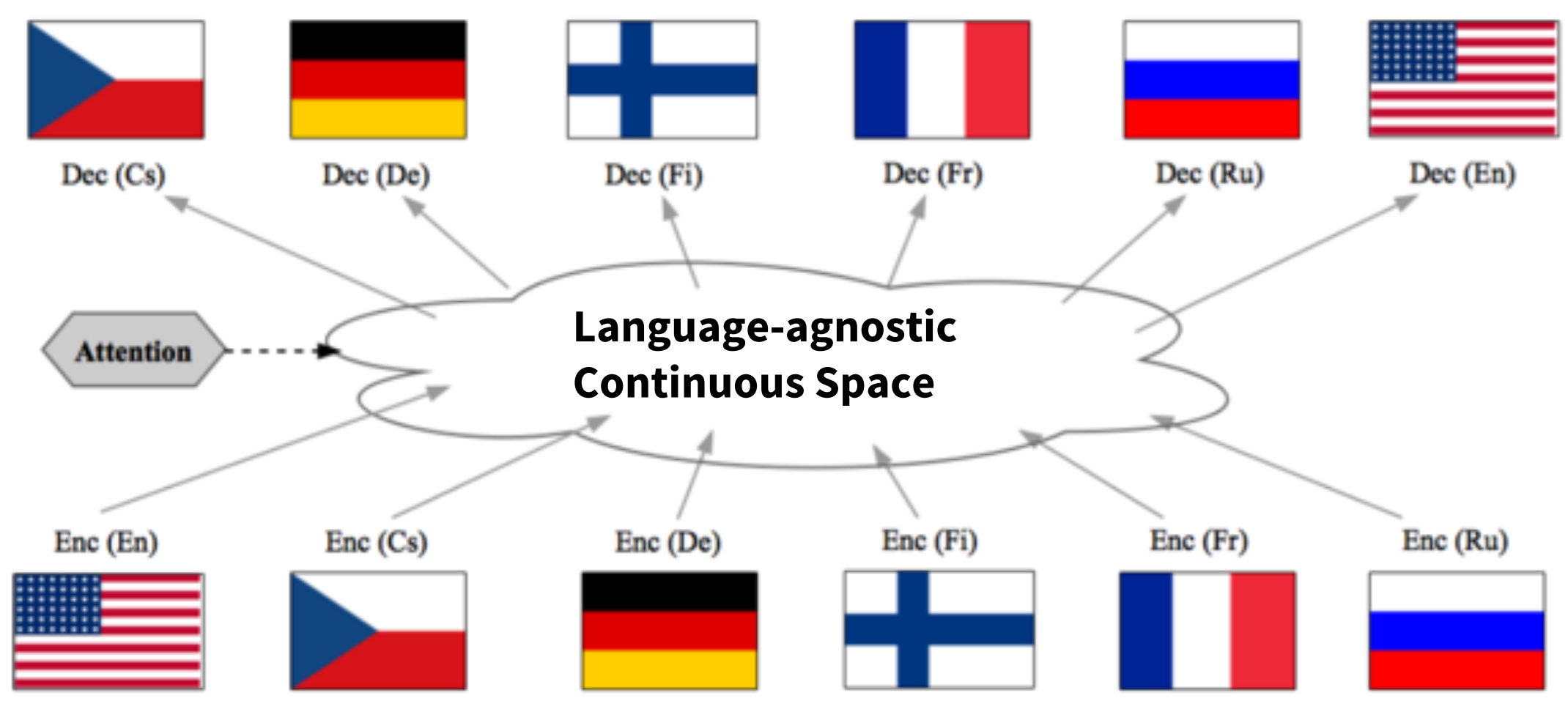

192 [Dong et al., ACL2015; Luong et al., ICLR2016; Firat et al., NAACL2016] 


\section{Multilingual Translation: Expectations}

1. Positive language transfer

2. \# of parameters grows linearly w.r.t. \# of languages

3. Multi-source translation [Zoph\&Knight, NAACL2016]

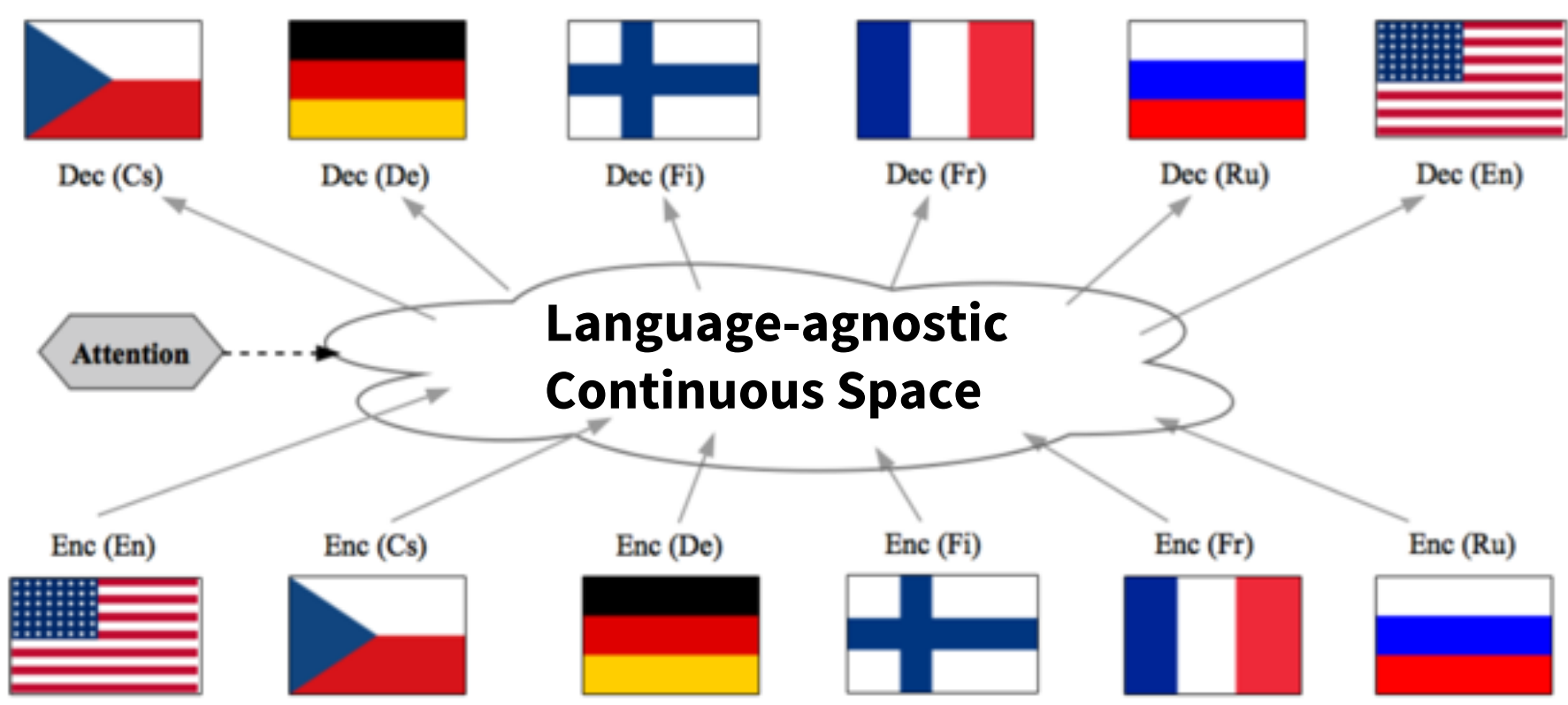

[Dong et al., ACL2015; Luong et al., ICLR2016; Firat et al., NAACL2016] 


\section{Multilingual Translation}

\section{with Shared Alignment}

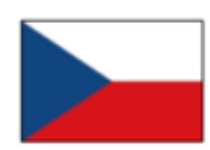

Dec (Cs)

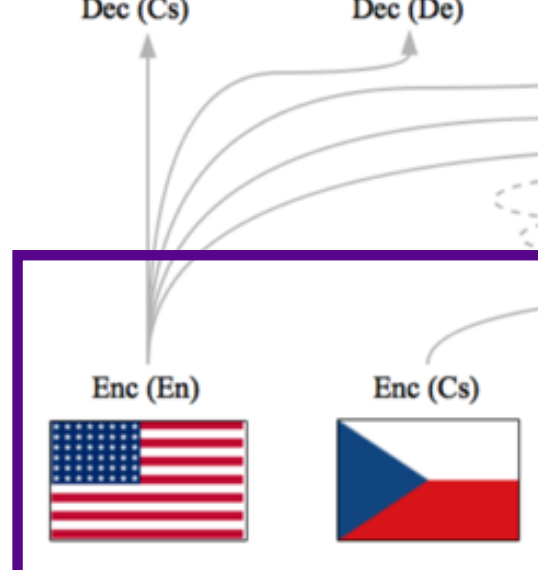

- Encoder per source language

Attention

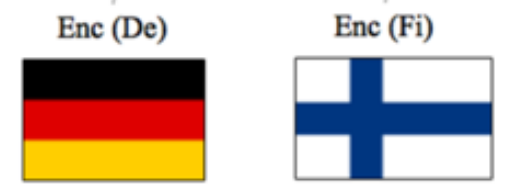

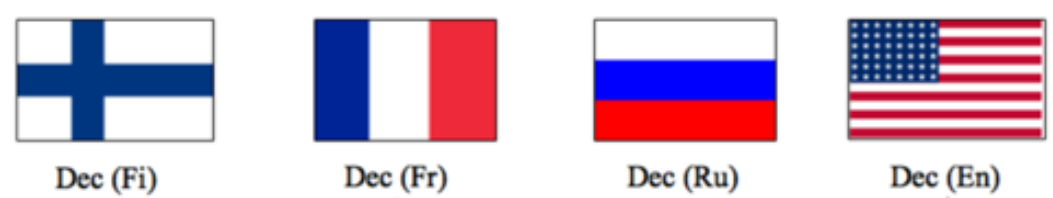

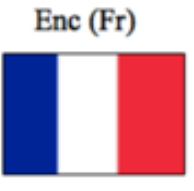

Enc (Ru)

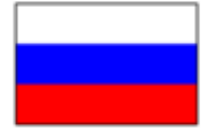

- Seq. of source symbols $\rightarrow$ Seq. of context vectors 


\section{Multilingual Translation}

\section{with Shared Alignment}

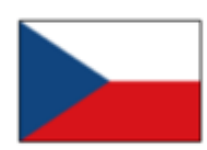

Dec (Cs)

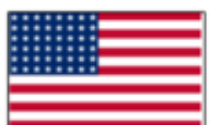

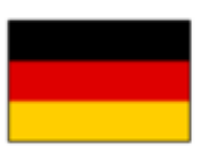

Dec (De)

A
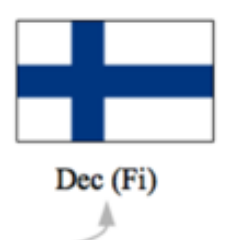

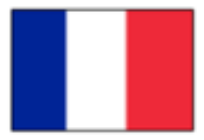

$\operatorname{Dec}(\mathrm{Fr})$

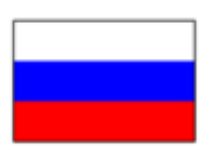

$\operatorname{Dec}(\mathrm{Ru})$

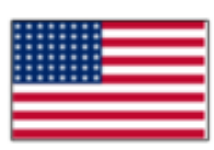

$\operatorname{Dec}(\mathrm{En})$
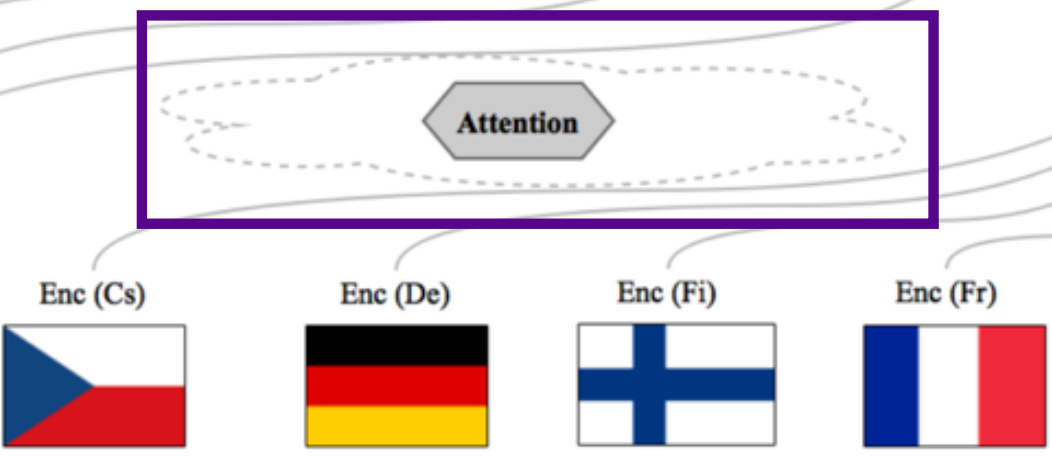

Enc (Ru)

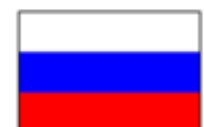

- Shared Attention Mechanism

- Target hidden state, source context vector $\rightarrow$ Attention weight 


\section{Multilingual Translation}

\section{with Shared Alignment}

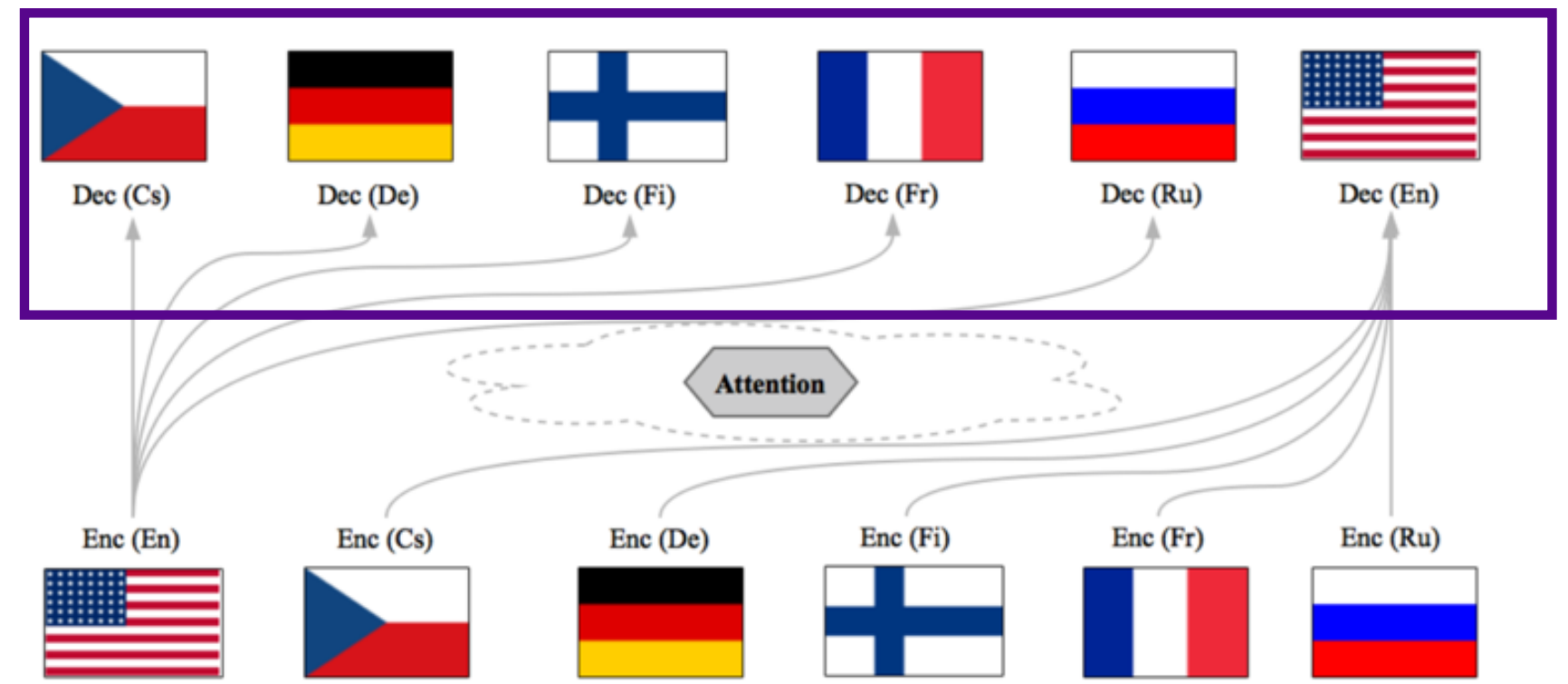

- Decoder per target language

- Aligned context vector $\rightarrow$ Target symbol 


\section{Multilingual Translation: Training}

- No multi-way parallel corpus assumed

- Bilingual sentence pairs only

- Each sentence pair activates/updates one encoder, decoder and shared attention

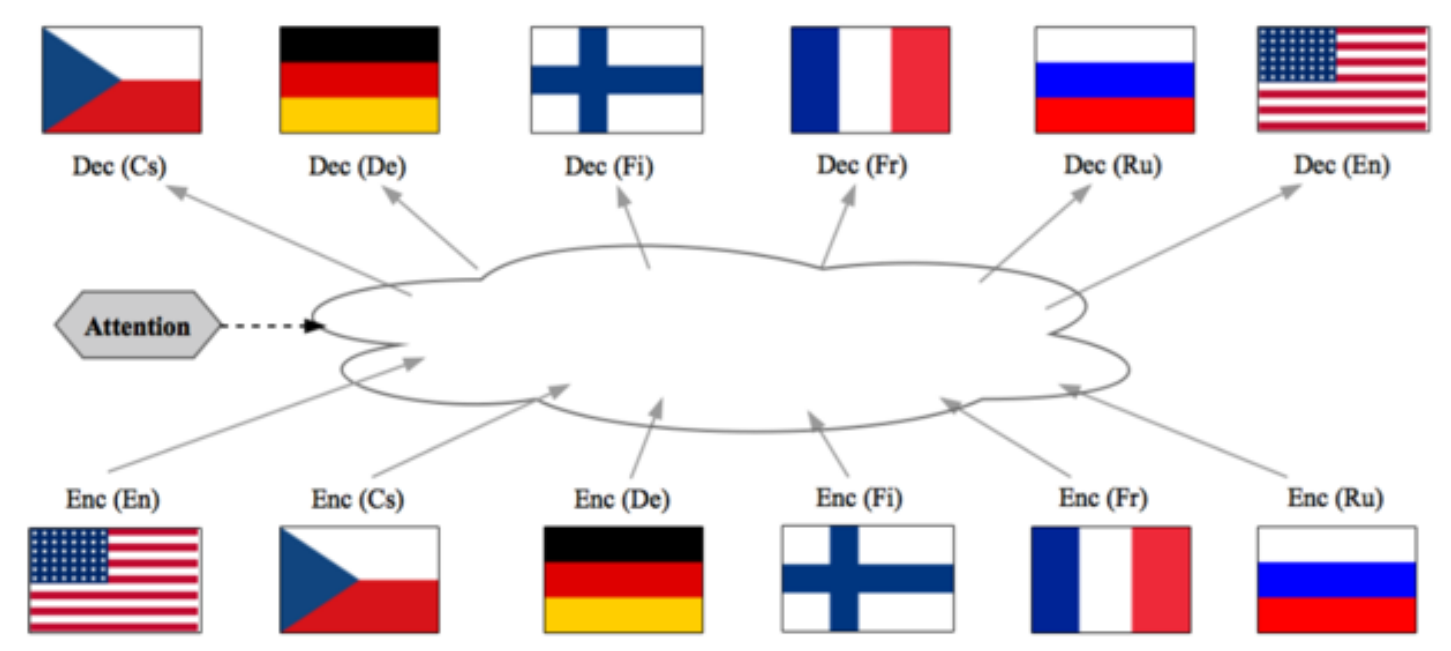




\section{Multilingual Translation: First Result}

- 10 language pair-directions

- $\mathrm{En} \rightarrow\{\mathrm{Fr}, \mathrm{Cs}, \mathrm{De}, \mathrm{Ru}, \mathrm{Fi}\}+\{\mathrm{Fr}, \mathrm{Cs}, \mathrm{De}, \mathrm{Ru}, \mathrm{Fi}\} \rightarrow \mathrm{En}$

- 60+ million bilingual sentence pairs

- Comparable to 10 single-pair models

To English

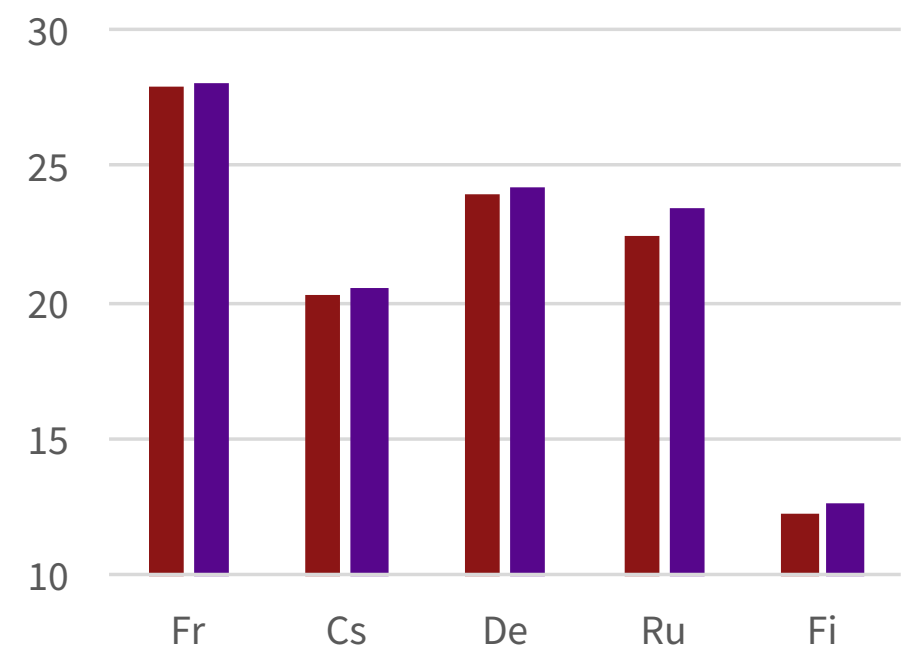

From English

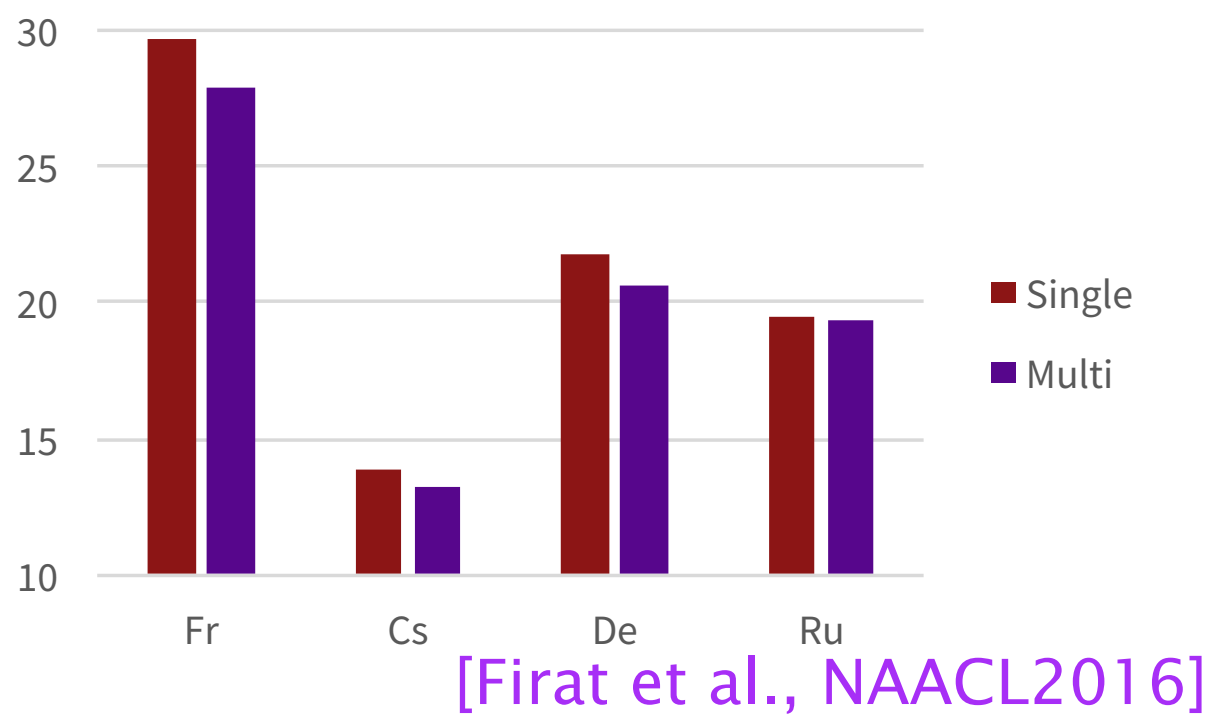




\section{Multilingual Translation: Looking Ahead}

- Low-resource translation

- Positive language transfer from high-resource to low-resource language pair-directions

\begin{tabular}{|c|c|c|c|c|c|}
\hline & \multicolumn{2}{|c|}{ \# Symbols } & \multicolumn{3}{|c|}{ \# Sentence } \\
\hline & \# En & Other & Train & Dev & Test \\
\hline En-Uz & $1.361 \mathrm{~m}$ & $1.186 \mathrm{~m}$ & $73.66 \mathrm{k}$ & 948 & 882 \\
\hline En-Es & $908.1 \mathrm{~m}$ & $924.9 \mathrm{~m}$ & $34.71 \mathrm{~m}$ & 3003 & 3000 \\
\hline$+\div$ En-Fr & $1.837 b$ & $1.911 \mathrm{~b}$ & $65.77 \mathrm{~m}$ & 3003 & 3000 \\
\hline
\end{tabular}




\section{Multilingual Translation: Looking Ahead}

- Low-resource translation: Example

Uz-En: 6.45

Uz-En + Tr-En: 9.34

Uz-En + Tr-En + Es-En: 10.34

Uz-En + Tr-En + Es-En + En-Tr: 9.41

Ensemble: 12.99

- 3x Uz-En + Tr-En + Es-En

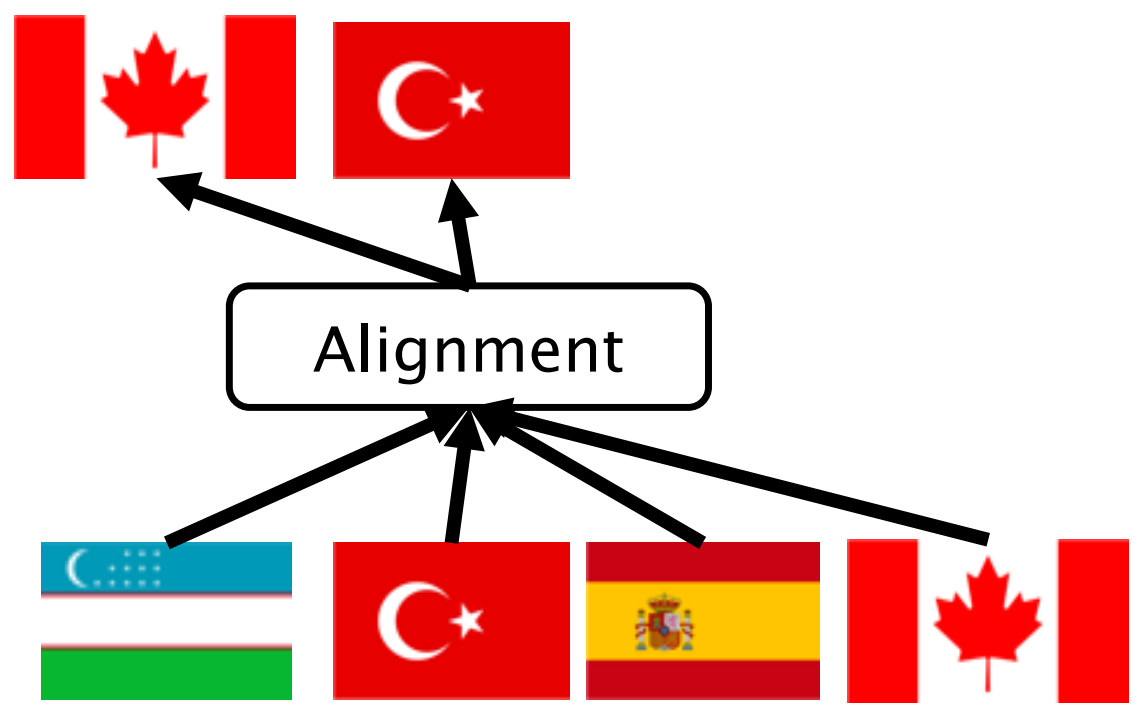

- 3x Uz-En + Tr-En + Es-En + En-Tr 


\section{Multilingual Translation: Looking Ahead}

- Zero-resource translation

- Translation without any direct parallel resource

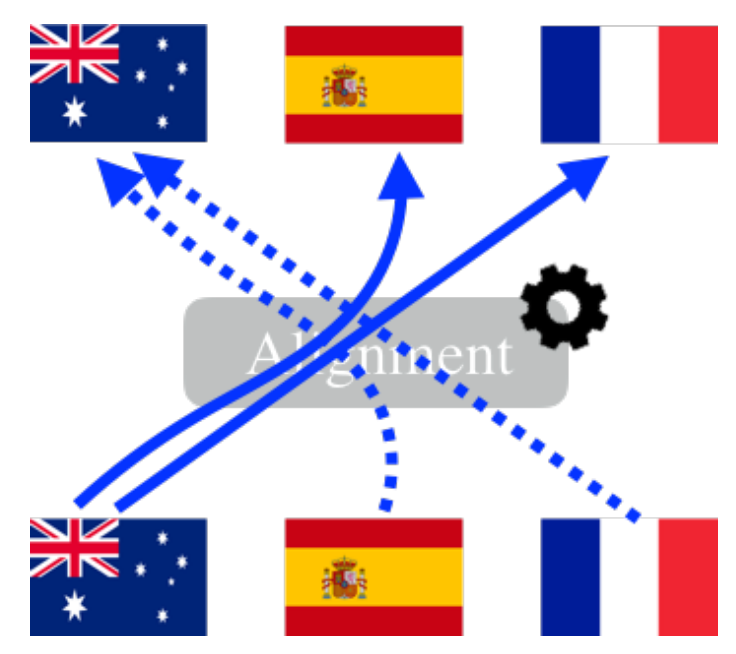

Training

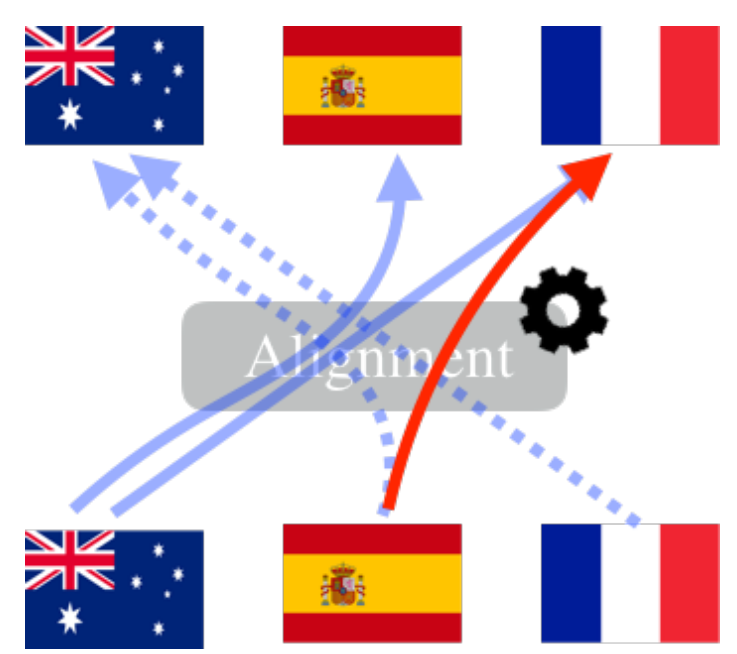

Testing 


\section{Multilingual Translation: Looking Ahead}

- Zero-resource translation

- Finetuning with pseudo-parallel corpus

[Sennrich et al., ACL2016]

- Closely related to unsupervised learning

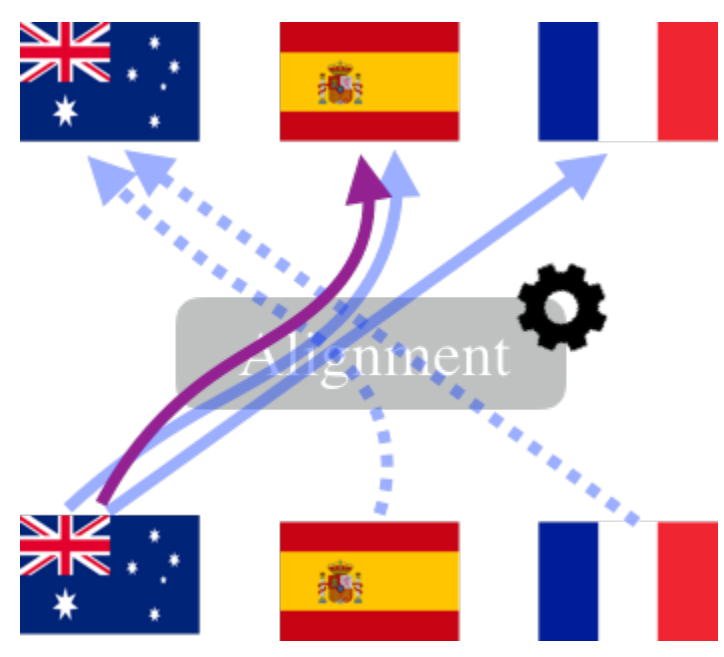

Pseudo-corpus Generation

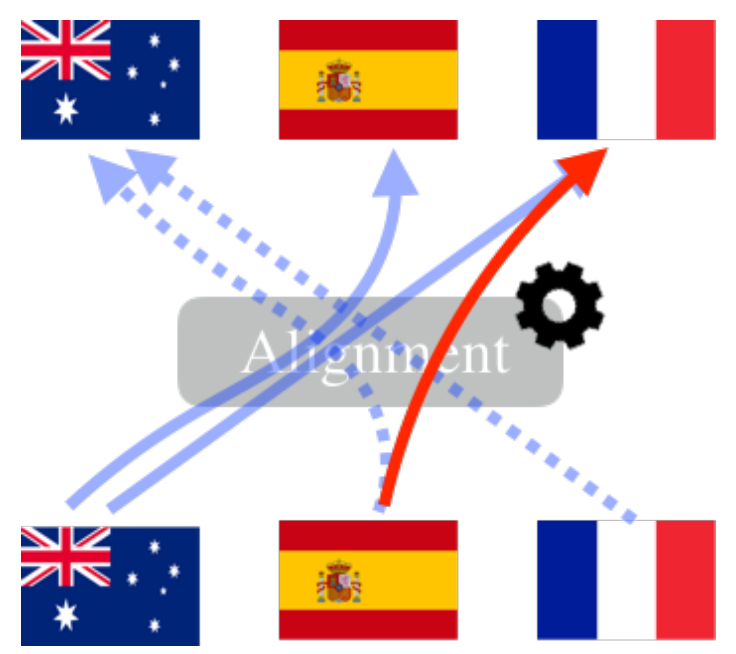

Finetuning

[Firat et al., EMNLP2016] 


\section{Multilingual Translation: Looking Ahead}

- Zero-resource translation

- Some initial result, but long way to go...

\begin{tabular}{|c|c|c|c|c|c|c|c|c|c|c|}
\hline \multirow[b]{2}{*}{ Pivot } & \multirow{2}{*}{\multicolumn{2}{|c|}{ Many-to-1 }} & \multicolumn{4}{|c|}{ Pseudo Parallel Corpus } & \multicolumn{4}{|c|}{ True Parallel Corpus } \\
\hline & & & $1 \mathrm{k}$ & $10 \mathrm{k}$ & $100 \mathrm{k}$ & $1 \mathrm{~m}$ & $1 \mathrm{k}$ & $10 \mathrm{k}$ & $100 \mathrm{k}$ & $1 \mathrm{~m}$ \\
\hline$\sqrt{ }$ & \multicolumn{2}{|c|}{ No Finetuning } & \multicolumn{4}{|c|}{ Dev: 20.64, Test 20.4} & \multicolumn{4}{|c|}{-} \\
\hline & & Dev & 0.28 & 10.16 & 15.61 & 17.59 & 0.1 & 8.45 & 16.2 & 20.59 \\
\hline & & Test & 0.47 & 10.14 & 15.41 & 17.61 & 0.12 & 8.18 & 15.8 & 19.97 \\
\hline \multirow{2}{*}{$\sqrt{ }$} & \multirow{2}{*}{ Early } & Dev & 19.42 & 21.08 & 21.7 & 21.81 & 8.89 & 16.89 & 20.77 & 22.08 \\
\hline & & Test & 19.43 & 20.72 & 21.23 & 21.46 & 9.77 & 16.61 & 20.40 & 21.7 \\
\hline \multirow{2}{*}{$\sqrt{ }$} & Early+ & Dev & 20.89 & 20.93 & 21.35 & 21.33 & 14.86 & 18.28 & 20.31 & 21.33 \\
\hline & Late & Test & 20.5 & 20.71 & 21.06 & 21.19 & 15.42 & 17.95 & 20.16 & 20.9 \\
\hline
\end{tabular}




\section{Multilingual Translation: Looking Ahead}

- Multi-modal, Multitask Translation

[Luong et al., ICLR2016; Caglayan et al., WMT2016]

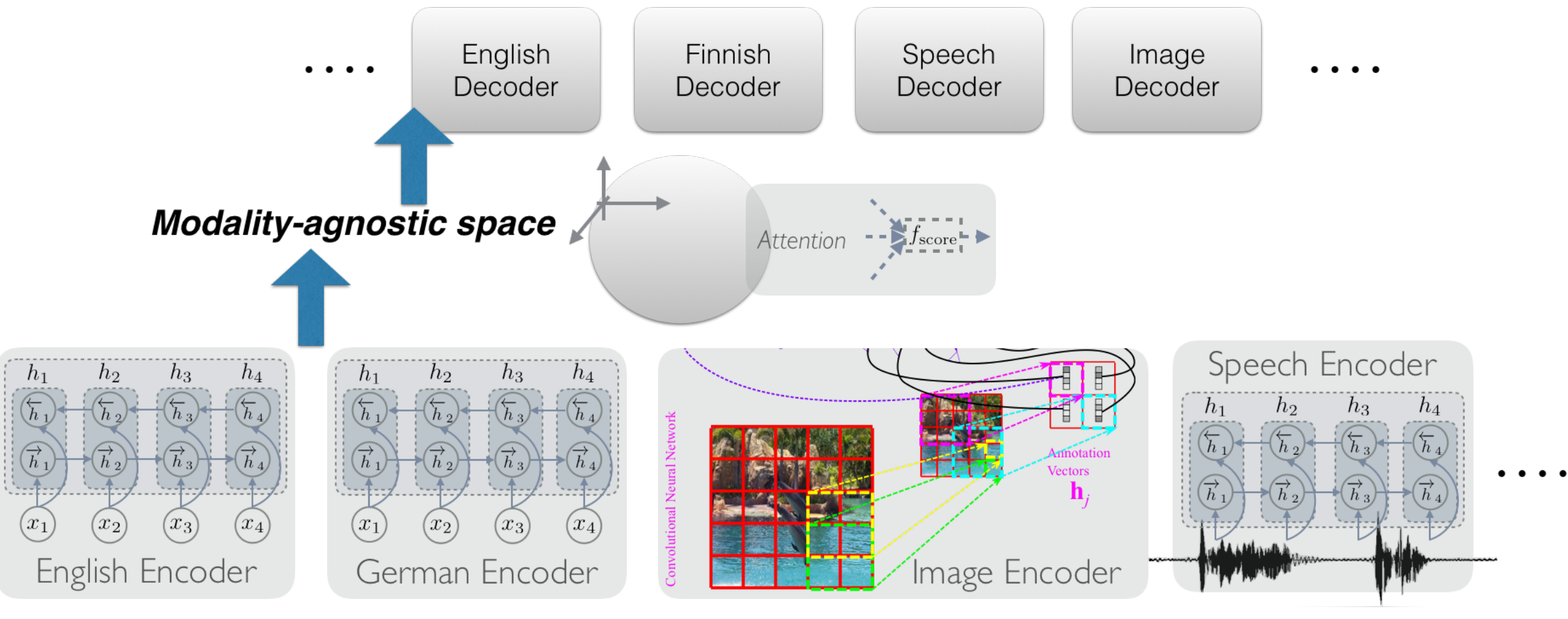




\section{Future of NMT}

a. Multi-task learning

b. Larger context

c. Mobile devices

d. Beyond Maximum Likelihood Estimation 


\section{Larger-context NMT}

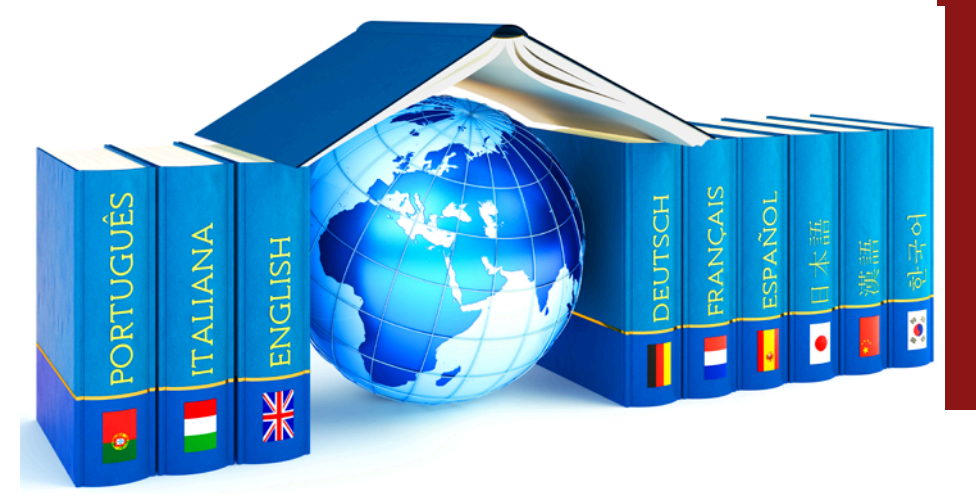

- Beyond sentence level:

- Paragraphs, articles, books, etc.

- Challenges?

- Extremely long sequences.

- Maintain across sentences:

- Coherent style

- Discourse structure 


\section{Solution: Hierarchical architectures?}

- Effective attention mechanism for long sequences 


\section{Solution: Hierarchical architectures?}

- Speech recognition [Chan, Jaity, Le, Vinyals, ICASSP'15].

Speller
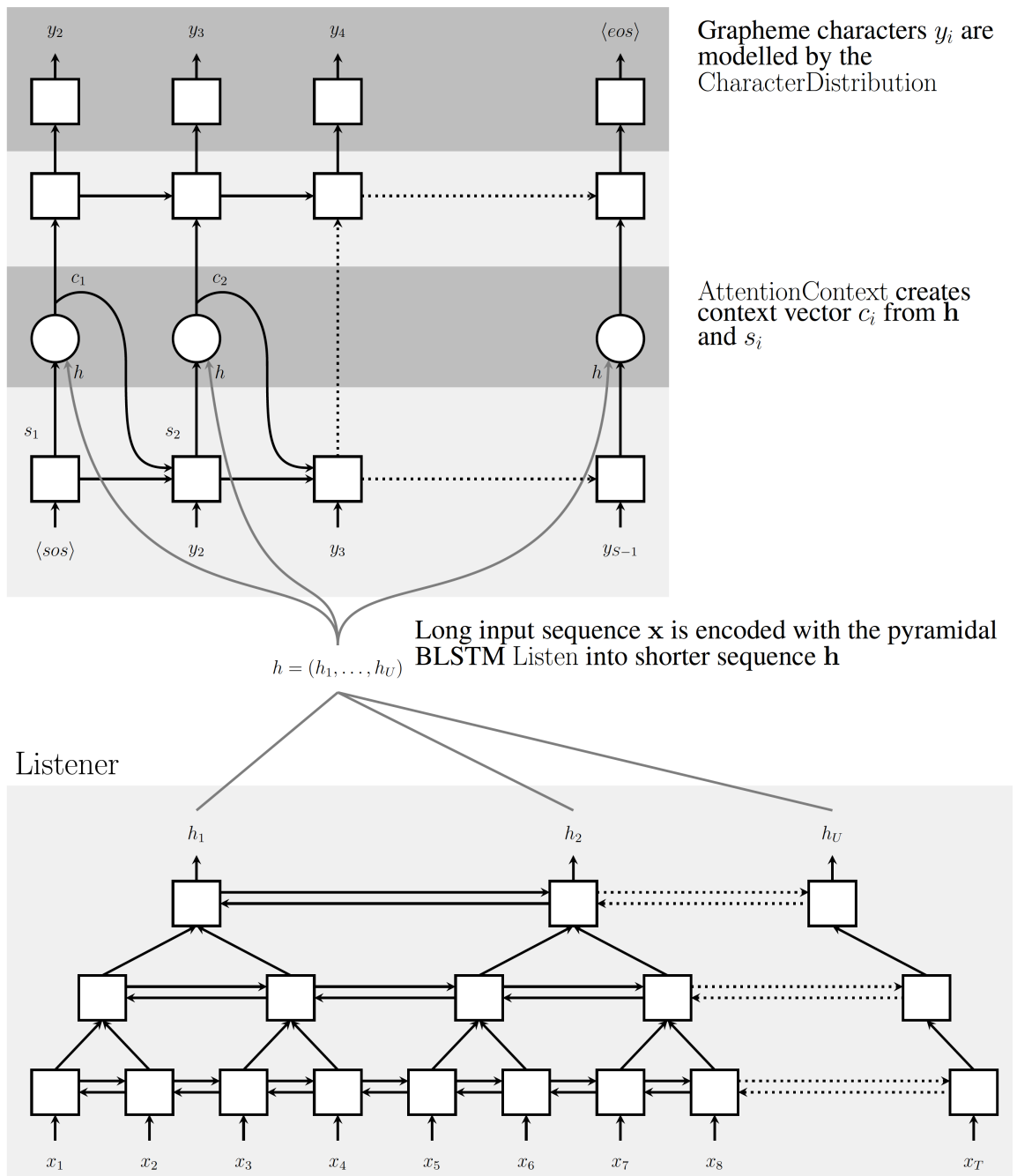


\section{Solution: Hierarchical architectures?}

- Speech recognition [Chan, Jaity, Le, Vinyals, ICASSP'15].

Speller
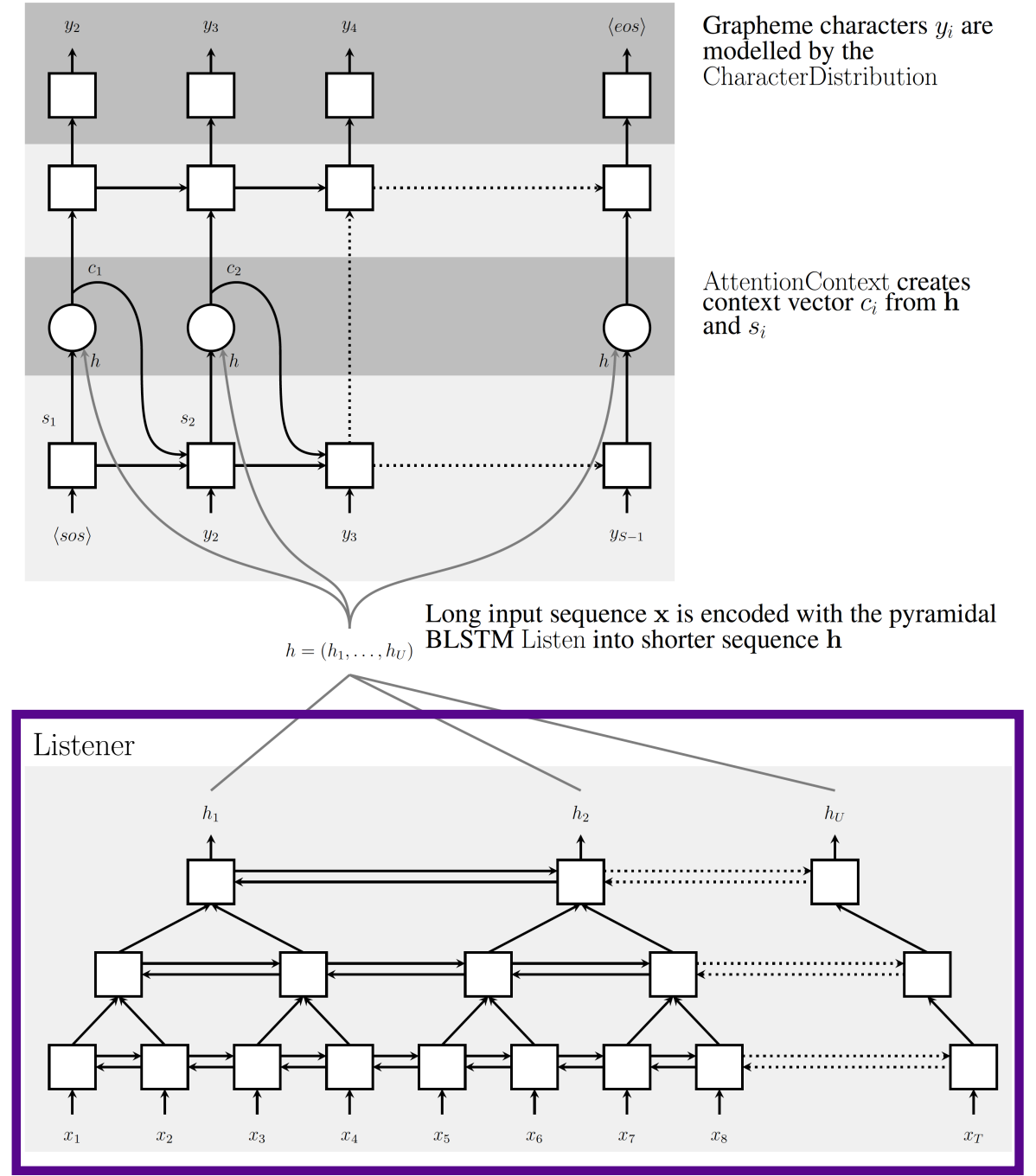

Speech signals: thousands of frames

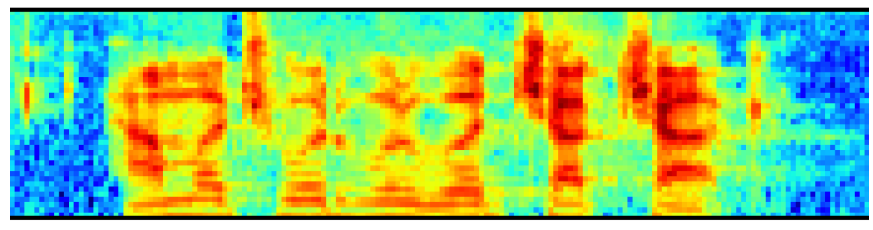




\section{Solution: Hierarchical architectures?}

- Speech recognition [Chan, Jaity, Le, Vinyals, ICASSP'15].

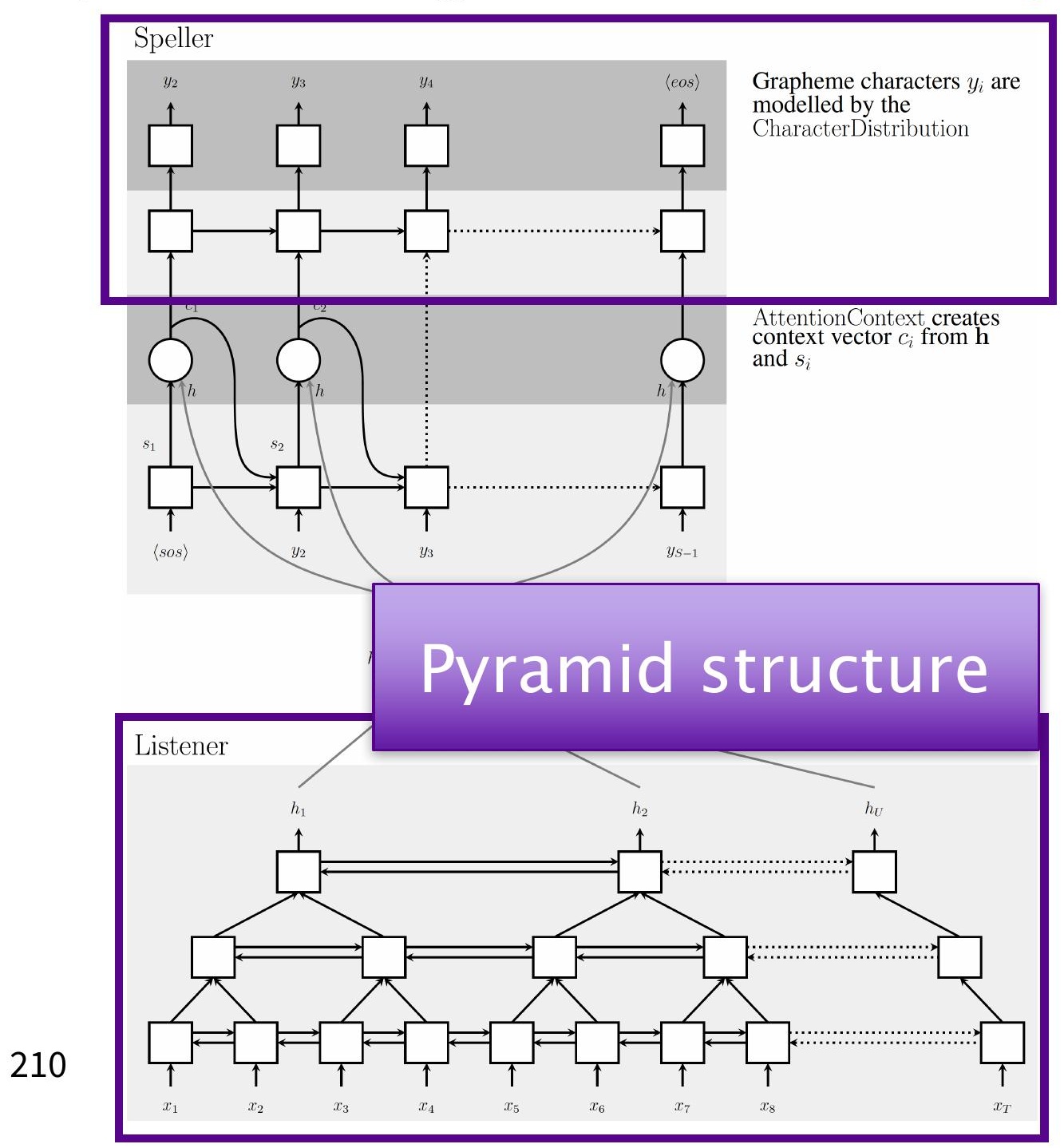

Speech transcription: "how much would a woodchuck chuck"

Speech signals: thousands of frames

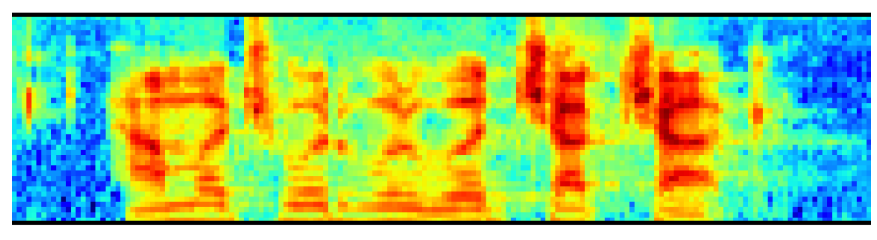




\section{Solution: Hierarchical architectures?}

- Effective attention mechanism for long sequences

- Speech recognition [Chan, Jaity, Le, Vinyals, ICASSP'15].

- Tracking states over time 


\section{Solution: Hierarchical architectures?}

- Dialogue systems [Serban, Sordoni, Bengio, Courville, Pineau , AAAl'15].

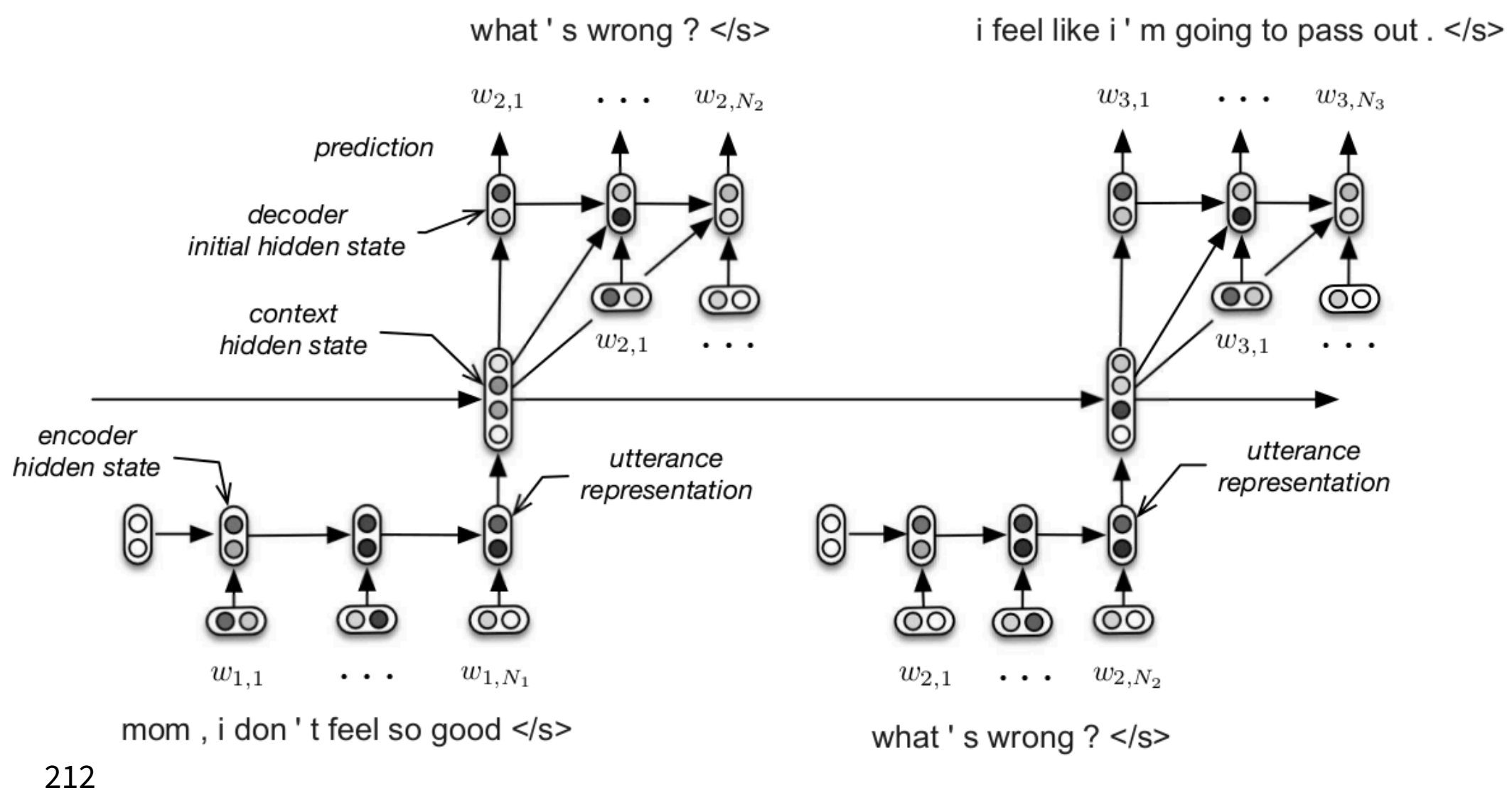




\section{Solution: Hierarchical architectures?}

- Dialogue systems [Serban, Sordoni, Bengio, Courville, Pineau , AAAl'15].

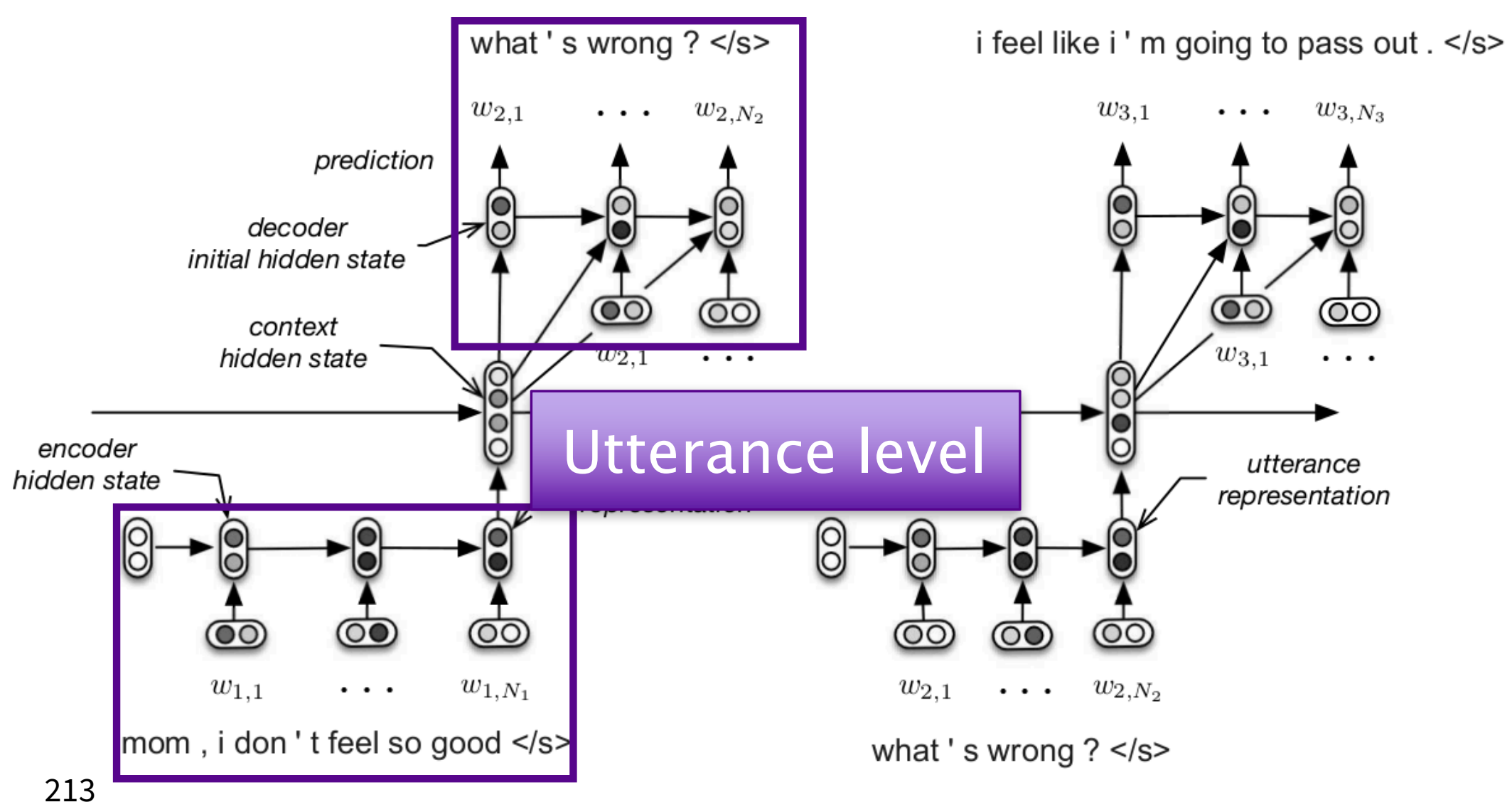




\section{Solution: Hierarchical architectures}

- Dialogue systems [Serban, Sordoni, Bengio, Courville, Pineau, AAAl'15].

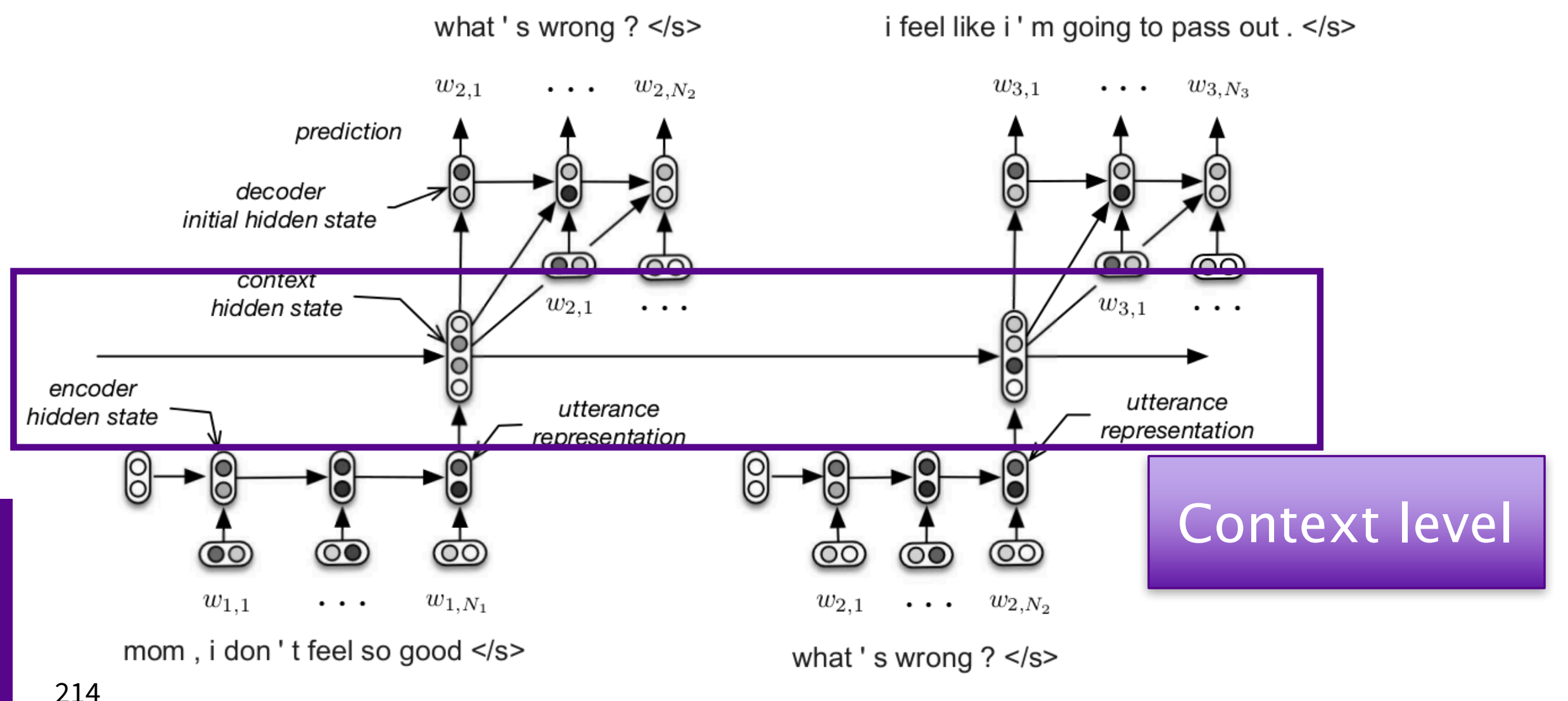




\section{Solution: Hierarchical architectures?}

- Effective attention mechanism for long sequences

- Speech recognition [Chan, Jaity, Le, Vinyals, ICASSP'15].

- Tracking states over many sentences

- Dialogue systems [Serban, Sordoni, Bengio, Courville, Pineau , AAAl'15].

\section{What else?}




\section{Future of NMT}

a. Multi-task learning

b. Larger context

c. Mobile devices

d. Beyond Maximum Likelihood Estimation 


\section{Mobile devices}

8 INDEPENDEN'T News voices Sports Culture Lifestyle Tech US election Daily Edition

\section{There are officially more mobile devices than people in the world}

The world is home to 7.2 billion gadgets, and they're multiplying five times faster than we are

- NMT has small memory footprint:

- No gigantic phrase tables \& LMs compared to SMT.

- Still, require large NNs for SOTA results

\section{Can we}

address this? 


\section{Model Pruning}

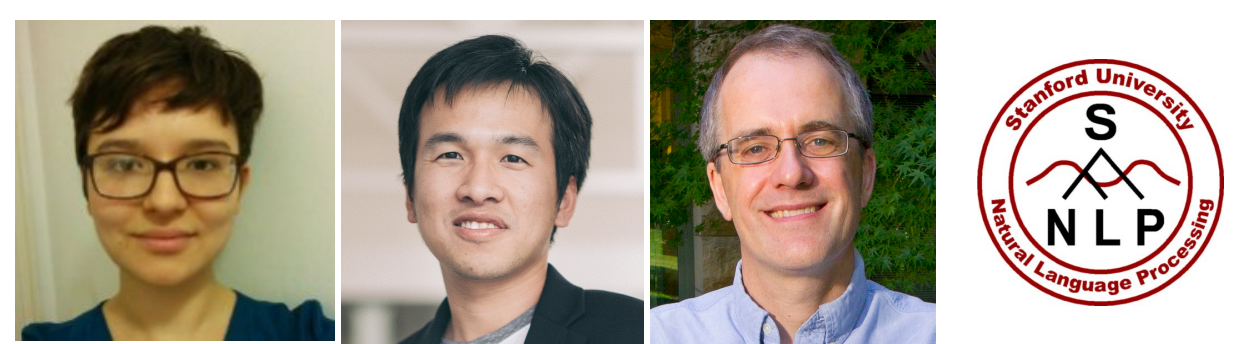

- NMT redundancy via pruning \& retraining:

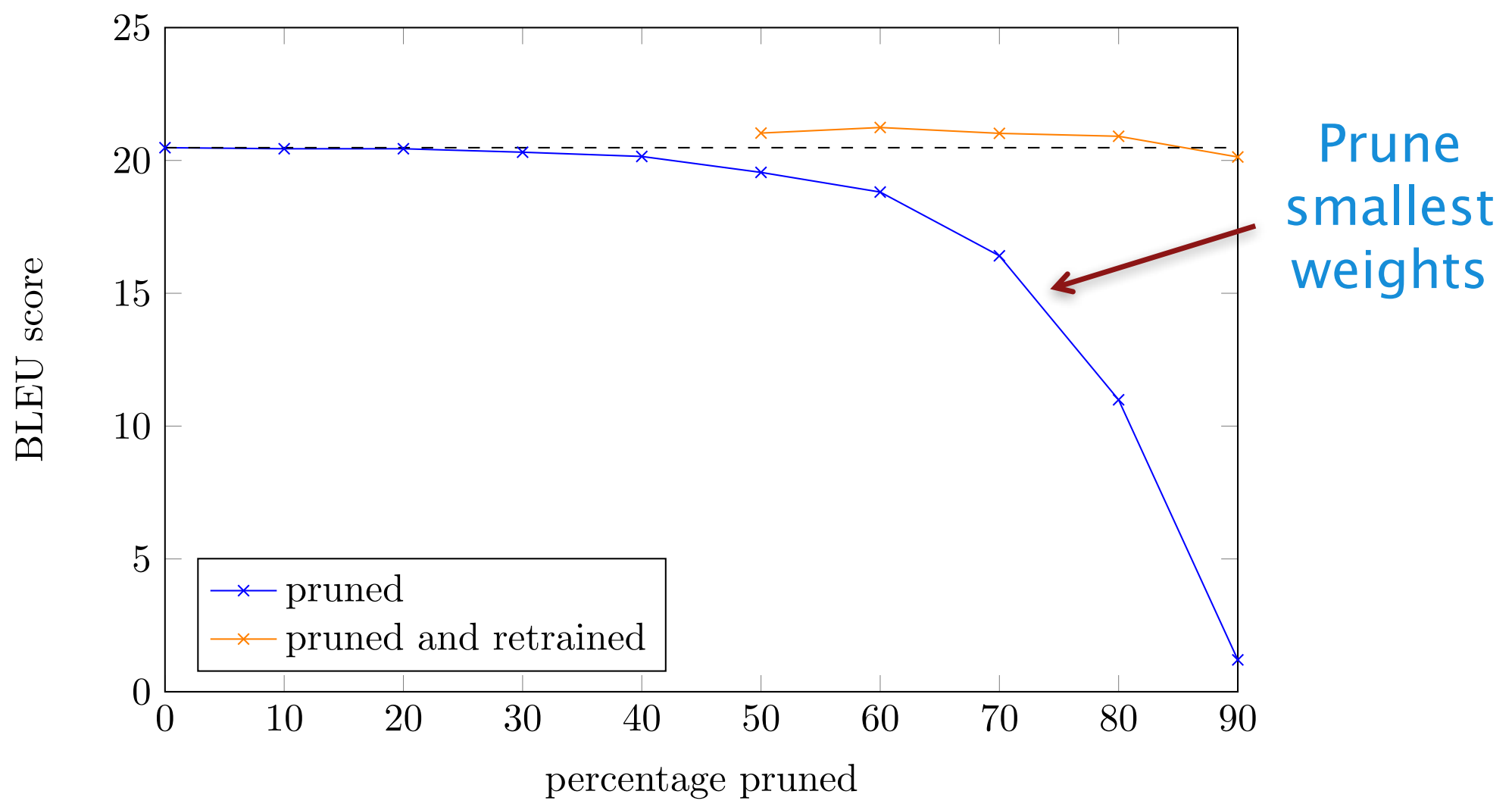

Abigail See, Thang Luong, Chris Manning. Compression of Neural Machine Translation Models via Pruning. CONLL'16. 
It was just a baby!

\section{Next, really putting NMT on to mobile devices!}




\section{Knowledge Distillation}

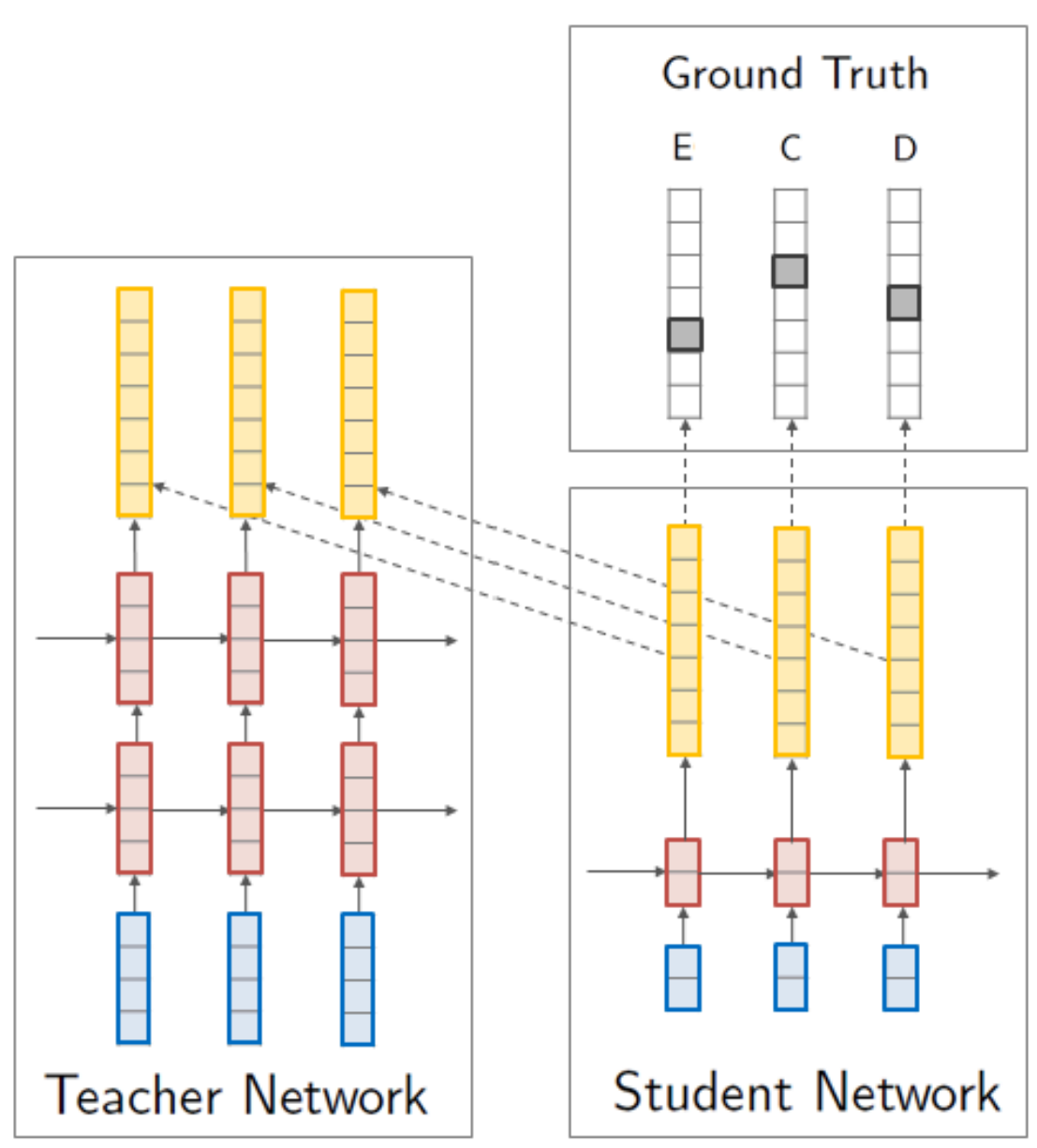

Yoon Kim, Alexander M. Rush. 


\section{Knowledge Distillation}
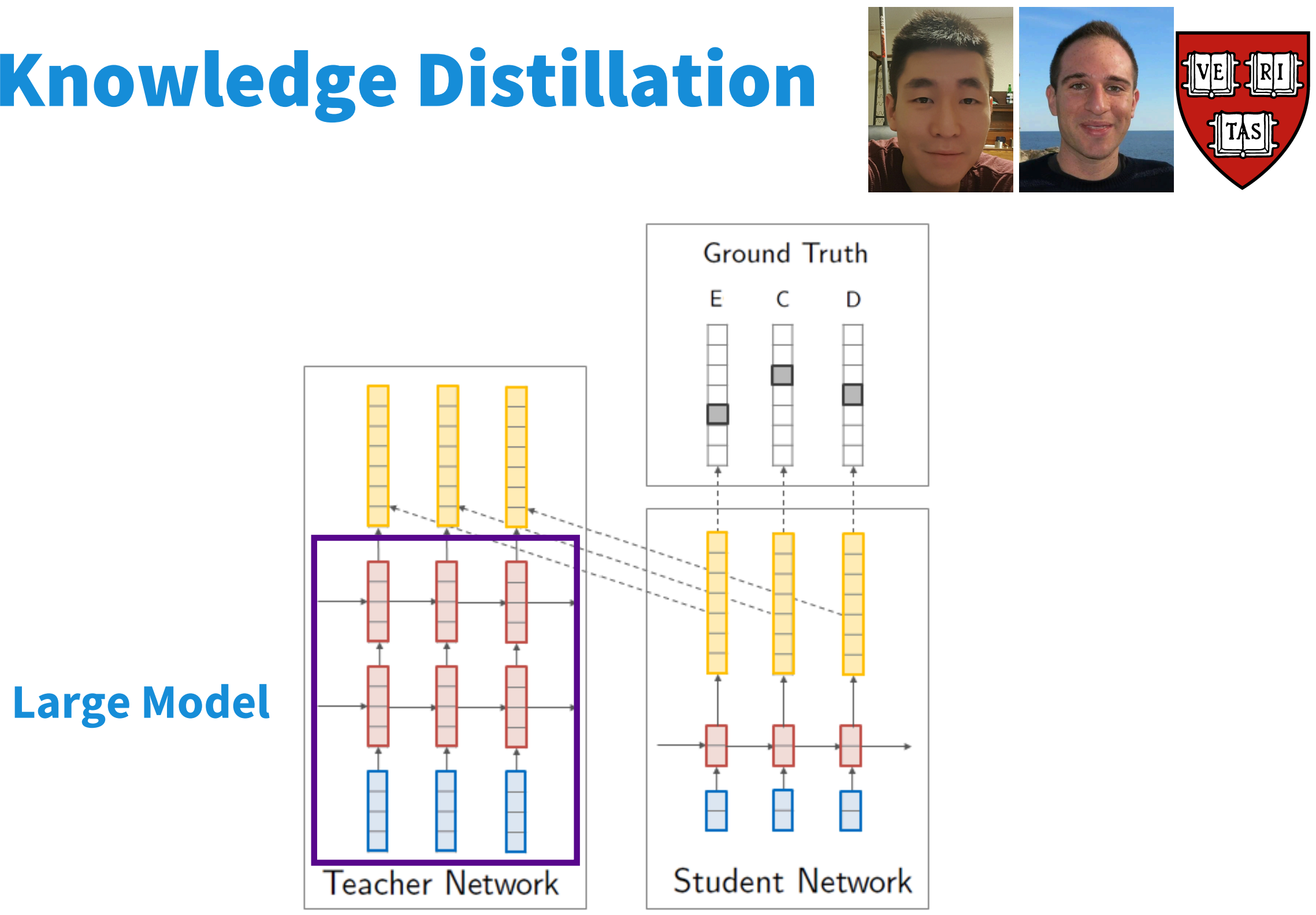

Yoon Kim, Alexander M. Rush. 


\section{Knowledge Distillation}
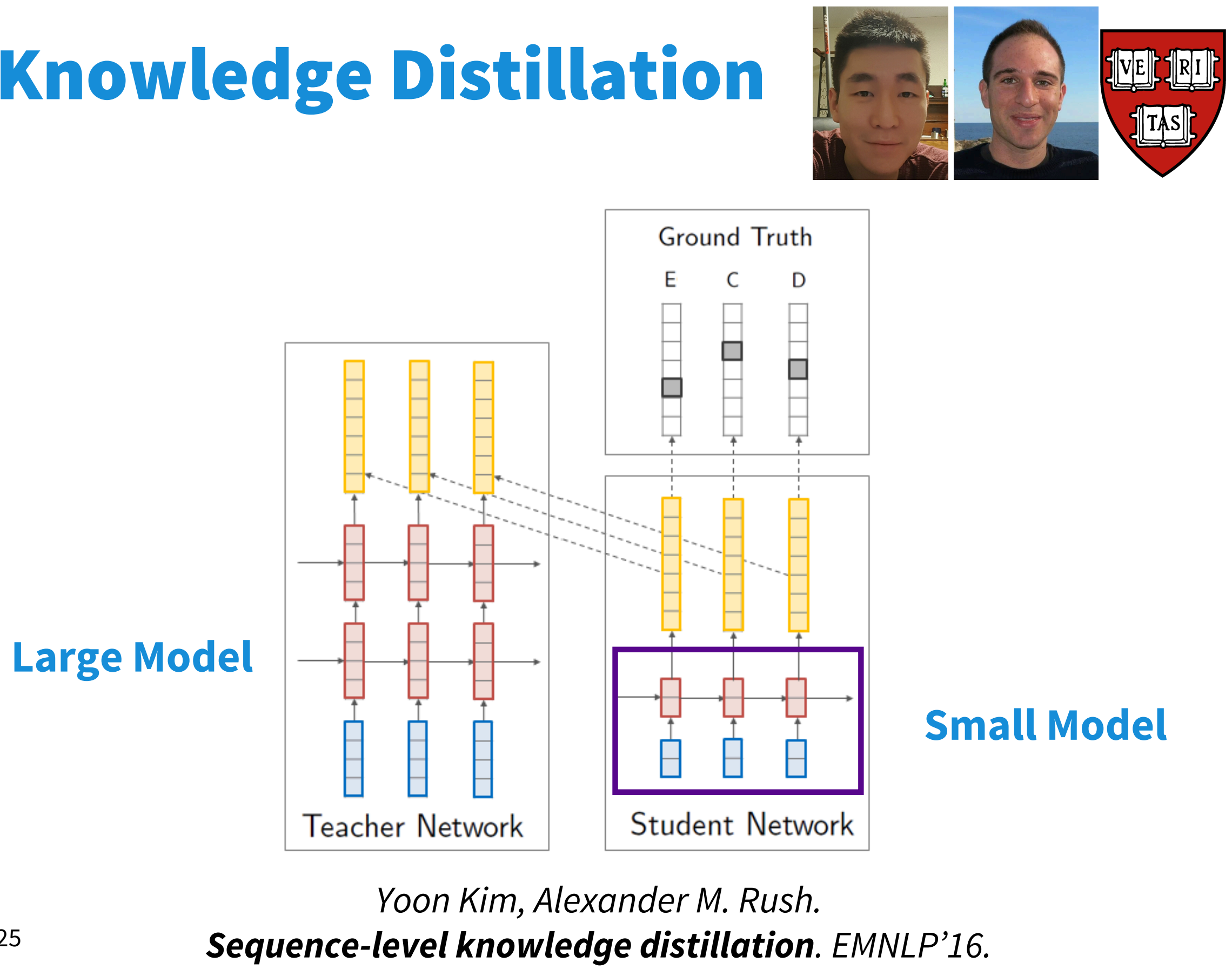


\section{Knowledge Distillation}
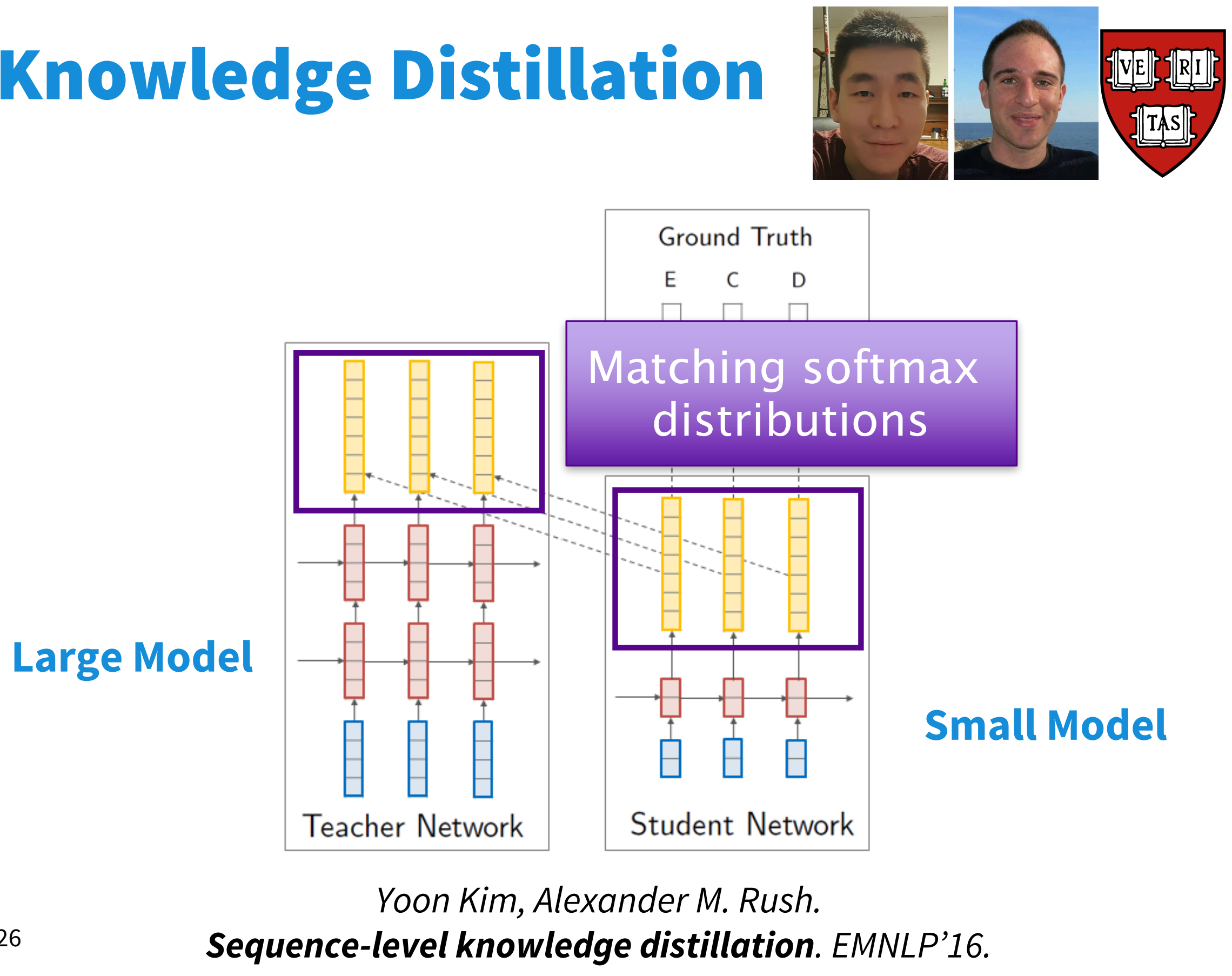


\section{Knowledge Distillation}

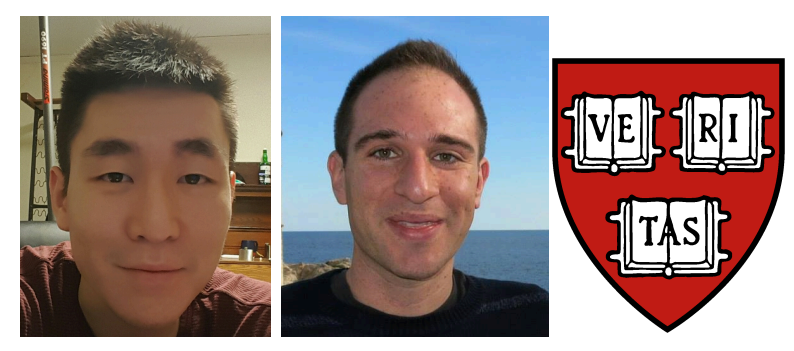

- Sequence-level knowledge distillation:

- Match the final distribution over sequences

- Beam search to create new training data

- Student model: no need beam search.

\section{0 times faster with only 0.2 BLEU loss!}

\section{https://github.com/harvardnlp/nmt-android}

Yoon Kim, Alexander M. Rush.

Sequence-level knowledge distillation. EMNLP'16. 


\section{Future of NMT}

a. Multi-task learning

b. Larger context

c. Mobile devices

d. Beyond Maximum Likelihood Estimation 


\section{Maximum Likelihood Estimation}

for Sequence Modelling

- Given a ground-truth trajectory, maximize the predictability of a next action: $\max \log p\left(x_{t} \mid x_{<t}\right)$

- Maximum (log-)likelihood estimation

- Two issues

1. Weak correlation with a true reward

2. Mismatch between training and

$p$ (the, cat, is, eating) inference
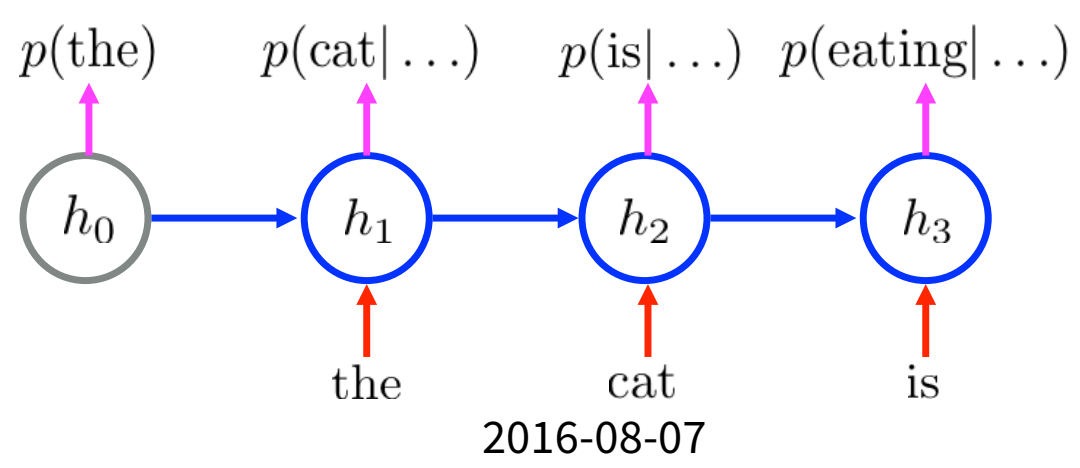


\section{Beyond Maximum Likelihood}

- Maximize the sequence-wise global loss

- Incorporate inference into training

- Stochastic inference

- Policy gradient [Ranzato et al., ICLR2016; Bahdanau et al., arXiv2016]

- Minimum risk training [Shen et al., ACL2016]

- Deterministic inference

- Learning to search [Wiseman \& Rush, arXiv2016]

Time Step

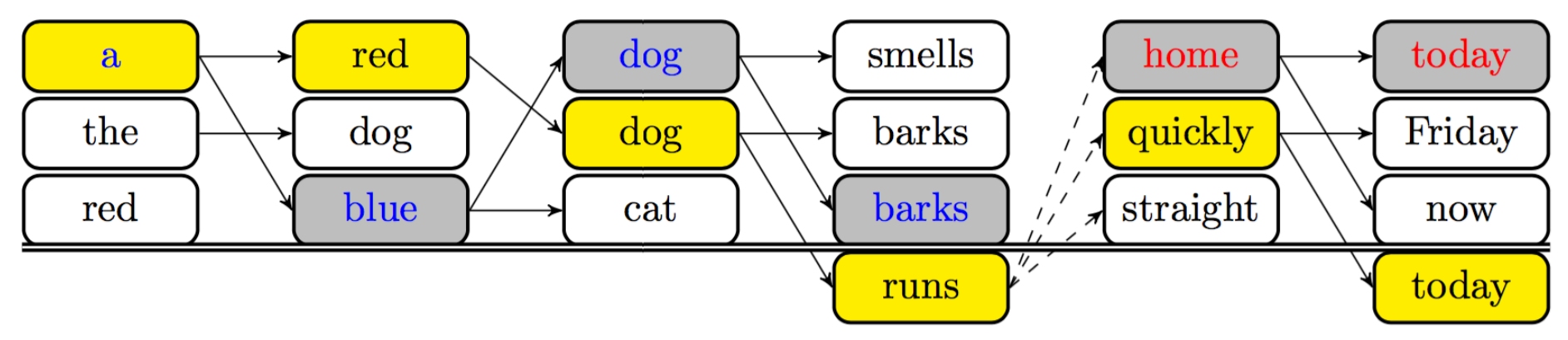




\section{What have we learnt today?}

1. History of MT and where Neural MT fits in

2. Language modelling \& Neural Machine Translation

a. Feedforward and recurrent language models

b. Recurrent neural network and its learning

c. Conditional language model: learning and decoding

3. Advanced Neural machine translation

a. Scaling softmax and copy mechanism

b. Attention-based models

c. Subword-level translation

d. Incorporating monolingual corpora

4. And, the future!

https://sites.google.com/site $231 / \mathrm{acl} 16 \mathrm{nmt} / \mathrm{home} /$ resources

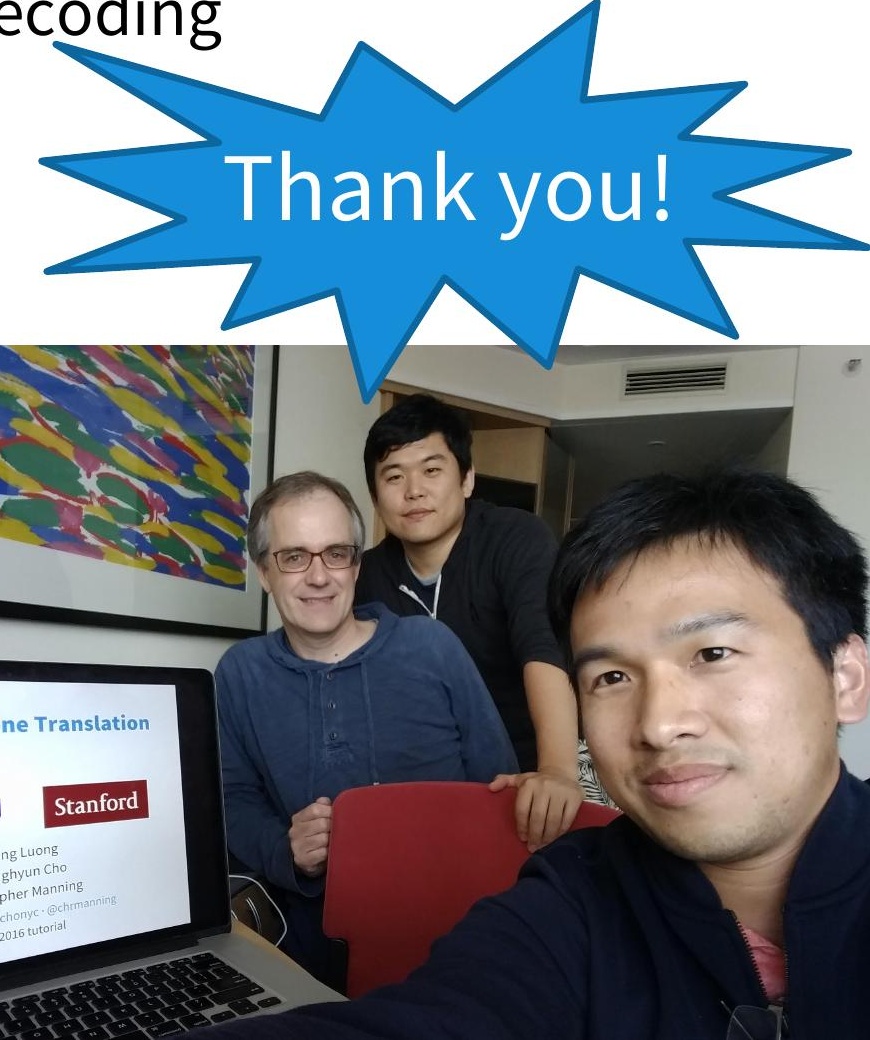




\section{References (1)}

- [Bahdanau et al., ICLR'15] Neural Translation by Jointly Learning to Align and Translate. http://arxiv.org/pdf/1409.0473.pdf

- [Chung, Cho, Bengio, ACL'16]. A Character-Level Decoder without Explicit Segmentation for Neural Machine Translation. http://arxiv.org/pdf/1603.06147.pdf

- [Cohn, Hoang, Vymolova, Yao, Dyer, Haffari, NAACL'16] Incorporating Structural Alignment Biases into an Attentional Neural Translation Model. https://arxiv.org/pdf/1601.01085.pdf

- [Dong, Wu, He, Yu, Wang, ACL'15]. Multi-task learning for multiple language translation. http://www.aclweb.org/anthology/P15-1166

- [Firat, Cho, Bengio, NAACL'16]. Multi-Way, Multilingual Neural Machine Translation with a Shared Attention Mechanism. https://arxiv.org/pdf/1601.01073.pdf

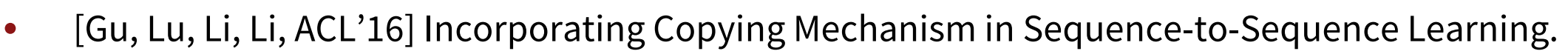
https://arxiv.org/pdf/1603.06393.pdf

- [Gulcehre, Ahn, Nallapati, Zhou, Bengio, ACL'16] Pointing the Unknown Words. http://arxiv.org/pdf/1603.08148.pdf

- $\quad$ Hochreiter \& Schmidhuber, 1997] Long Short-term Memory. http://deeplearning.cs.cmu.edu/pdfs/Hochreiter97_Istm.pdf

- $\quad$ Kim, Jernite, Sontag, Rush, AAAl'16]. Character-Aware Neural Language Models. https://arxiv.org/pdf/1508.06615.pdf 


\section{References (2)}

- [Ji, Haffari, Eisenstein, NAACL'16] A Latent Variable Recurrent Neural Network for Discourse-Driven Language Models. https://arxiv.org/pdf/1603.01913.pdf

- [Ji, Vishwanathan, Satish, Anderson, Dubey, ICLR'16] BlackOut: Speeding up Recurrent Neural Network Language Models with very Large Vocabularies. http://arxiv.org/pdf/1511.06909.pdf

- [Jia, Liang, ACL'16]. Data Recombination for Neural Semantic Parsing. https://arxiv.org/pdf/1606.03622.pdf

- $\quad$ [Ling, Luís, Marujo, Astudillo, Amir, Dyer, Black, Trancoso, EMNLP'15]. Finding Function in Form: Compositional Character Models for Open Vocabulary Word Representation. http://arxiv.org/pdf/1508.02096.pdf

- [Luong et al., ACL'15a] Addressing the Rare Word Problem in Neural Machine Translation. http://www.aclweb.org/anthology/P15-1002

- [Luong et al., ACL'15b] Effective Approaches to Attention-based Neural Machine Translation. https://aclweb.org/anthology/D/D15/D15-1166.pdf

- [Luong \& Manning, IWSLT'15] Stanford Neural Machine Translation Systems for Spoken Language Domain. http://nlp.stanford.edu/pubs/luong-manning-iwslt15.pdf

- $\quad$ [Mnih \& Hinton, NIPS’09] A Scalable Hierarchical Distributed Language Model.

https://www.cs.toronto.edu/ amnih/papers/hlbl final.pdf

- $\quad$ [Mnih \& Teh, ICML'12] A fast and simple algorithm for training neural probabilistic language models. https://www.cs.toronto.edu/ amnih/papers/ncelm.pdf

- [Mnih et al., NIPS'14] Recurrent Models of Visual Attention. http://papers.nips.cc/paper/5542-recurrent-modelsof-visual-attention.pdf

- $\quad$ [Morin \& Bengio, AISTATS’05] Hierarchical Probabilistic Neural Network Language Model. http://www.iro.umontreal.ca/ lisa/pointeurs/hierarchical-nnlm-aistats05.pdf 


\section{References (3)}

- [Sennrich, Haddow, Birch, ACL'16a]. Improving Neural Machine Translation Models with Monolingual Data. http://arxiv.org/pdf/1511.06709.pdf

- $\quad$ Sennrich, Haddow, Birch, ACL'16b]. Neural Machine Translation of Rare Words with Subword Units. http://arxiv.org/pdf/1508.07909.pdf

- $\quad$ [Sutskever et al., NIPS'14] Sequence to Sequence Learning with Neural Networks. http://papers.nips.cc/paper/5346-sequence-to-sequence-learning-with-neural-networks.pdf

- $\quad$ [Tu, Lu, Liu, Liu, Li, ACL'16] Modeling Coverage for Neural Machine Translation. http://arxiv.org/pdf/1601.04811.pdf

- [Vaswani, Zhao, Fossum, Chiang, EMNLP'13] Decoding with Large-Scale Neural Language Models Improves Translation. http://www.isi.edu/ avaswani/NCE-NPLM.pdf

- $\quad$ [Wang, Cho, ACL'16]. Larger-Context Language Modelling with Recurrent Neural Network. http://aclweb.org/anthology/P/P16/P16-1125.pdf

- $\quad$ Xu, Ba, Kiros, Cho, Courville, Salakhutdinov, Zemel, Bengio, ICML'15] Show, Attend and Tell: Neural Image Caption Generation with Visual Attention. http://jmlr.org/proceedings/papers/v37/xuc15.pdf

- [Zoph, Knight, NAACL'16]. Multi-source neural translation. http://www.isi.edu/naturallanguage/mt/multi-source-neural.pdf

- [Zoph, Vaswani, May, Knight, NAACL'16] Simple, Fast Noise Contrastive Estimation for Large RNN Vocabularies. http://www.isi.edu/natural-language/mt/simple-fast-noise.pdf 